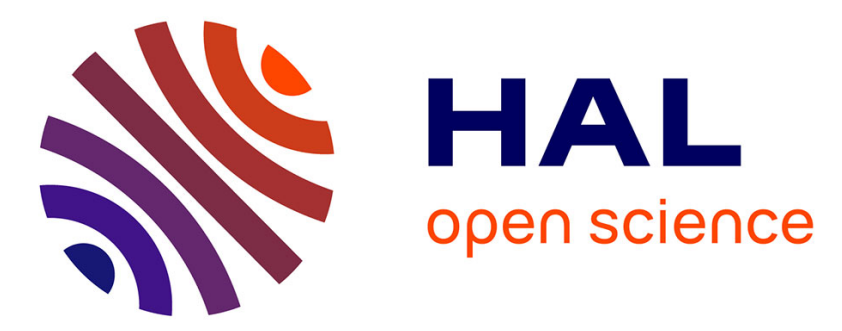

\title{
Des abords du forum au palais archiepiscopal : Étude du centre monumental d'Aix-en-Provence
}

\author{
Michel Fixot, Jean Guyon, Jean-Pierre Pelletier, Lucien Rivet
}

\section{To cite this version:}

Michel Fixot, Jean Guyon, Jean-Pierre Pelletier, Lucien Rivet. Des abords du forum au palais archiepiscopal: Étude du centre monumental d'Aix-en-Provence. Bulletin Monumental, 1986, 144 (3), pp.195-290. 10.3406/bulmo.1986.2829 . halshs-01744113

\section{HAL Id: halshs-01744113 \\ https://shs.hal.science/halshs-01744113}

Submitted on 16 Apr 2018

HAL is a multi-disciplinary open access archive for the deposit and dissemination of scientific research documents, whether they are published or not. The documents may come from teaching and research institutions in France or abroad, or from public or private research centers.
L'archive ouverte pluridisciplinaire HAL, est destinée au dépôt et à la diffusion de documents scientifiques de niveau recherche, publiés ou non, émanant des établissements d'enseignement et de recherche français ou étrangers, des laboratoires publics ou privés.

\section{(1)(1) $\$(0)$}

Distributed under a Creative Commons Attribution - NonCommercial - ShareAlikel 4.0 


\section{Des abords du forum au palais archiepiscopal [Étude du centre} monumental d'Aix-en-Provence ]

Michel Fixot, Jean Guyon, Jean-Pierre Pelletier, Lucien Rivet

\section{Citer ce document / Cite this document :}

Fixot Michel, Guyon Jean, Pelletier Jean-Pierre, Rivet Lucien. Des abords du forum au palais archiepiscopal [Étude du centre monumental d'Aix-en-Provence ]. In: Bulletin Monumental, tome 144, n³, année 1986. pp. 195-290;

doi : 10.3406/bulmo.1986.2829

http://www.persee.fr/doc/bulmo_0007-473x_1986_num_144_3_2829

Document généré le 07/11/2016 


\title{
DES ABORDS DU FORUM AU PALAIS ARCHIEPISCOPAL
}

\author{
ETUDE DU (FNTRE MONLIENTAI, D'AIX-FN-PROYFNCE
}

par Michel FIXOT, Jean GUYON, Jean-Pierre PELLETIER, Lucien RIVET

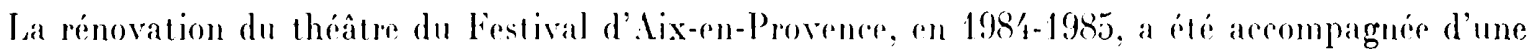
fouille daus la Cour de l'Archerèché qui rurichit nos comnaissances sur le palais archiépiscopal en mème temps qu'elle atteste, sur près de deux millénaires, la continuite de loncrupation d'un site qui a été de tout tempss an cour de l'agglomération aixoise (1) (fig. 1).

L'entreprise relevait an premier chef de l'archéologie monumentale - et cest à ce titre que les éditeurs du Bulletin monumental ont libéralement ourert leurs colonnes à la publication de celle fonille; mais est-il encore opératoire de distingure une archéologie monumentale quand la pratieque quotidienne montre l'unité profonde de l'arééologie l'archéologie tout court, saus qualificatif) et qu'elle interdit de dissocier l'étude du sous-sol de ceelle des élévations? le chantier de l'Archevèché nous a doméc l'occasion de le vérifier une nouvelle fois car c'est la combinaison desenseignements fournis par trois études complémentaires (celle des données dune fonille stratigraphique; celle des élements arehitecturanx conservés ou mis au jour; celle enfin des textes et des documents graphicpues anciens) (qui, senle, peut permettre de retracer une évolution presque continue, de l'urbanisme réglé de l'Antiquité aux ultimes transformations de l'époque moderne.

Dans un article, mème volumineux, il ne s'agit pourtant pas de récrire l'histoire du palais archiépiscopal (ni surtout celle de l'évolution urbaine d'ilix!) mats senlement de présenter des documents nouveaux et d'indicquer quel éclairage ils jeltent mème sur des points que l'on crovait bien élucidés; ce faisant, nous voulons moins apporter des réponses définitives cu'inciter à lat recherche - une recherche qui pourait progresser à l'avenir aussi par de nouvelles fouilles.

En suivant au plus press un ordre chronologicue, nous nous sommes donc efforés de constituer un dossier aussi précis et complet que possible ; encore convient-il d'indiquer préalalulement fuel est en possible.

Le dossier est précis parce que nous arons tenu à donner les pièces justificatives de nos interprétations (qu'elles viennent des études d'appareil, de la stratigraphie, de la céramicque ou des monnaies) et que nous n’avons dissimulé ni nos incertitudes ni nos hésitations; mais pour éviter de lasser le lecteur, nous avons réduit à l'essenticl cet apparat critique de l'archéologie, laissant du conp dans l'ombre toute une documentation que nous avons recueillie avec d'autant plus de soin que les vestiges mis an jour étajent vonés à la disparition : nous songeons par expmple à ces relevés à échelle 1/20\% que l'équipe aixoise de l'Institut de Recherche pour l'Architecture Antique a dressés au prix d'un travail continu de plusieurs mois, et dont les quelques plans reproduits ici ne donnent qu'une image très imparfaite $(2)$.

Le dossier est également complet - mais complet daus les limites de nos lacunes qui sont considérables : la fouille était commandée par l'urgence du chantier de rénovation ot même si elle a duré plus de trois mois, il s'en faut qu'elle ait été exhaustive. Certains secteurs moins radicalement menacés ont été laissés à l'écart de la recherche, d'autres ont été aménagés sous surveillance archéologique légère et mème là où l'enquète a été plus approfondie, la reconnaissance des différents niveaux est souvent restée rapide. 


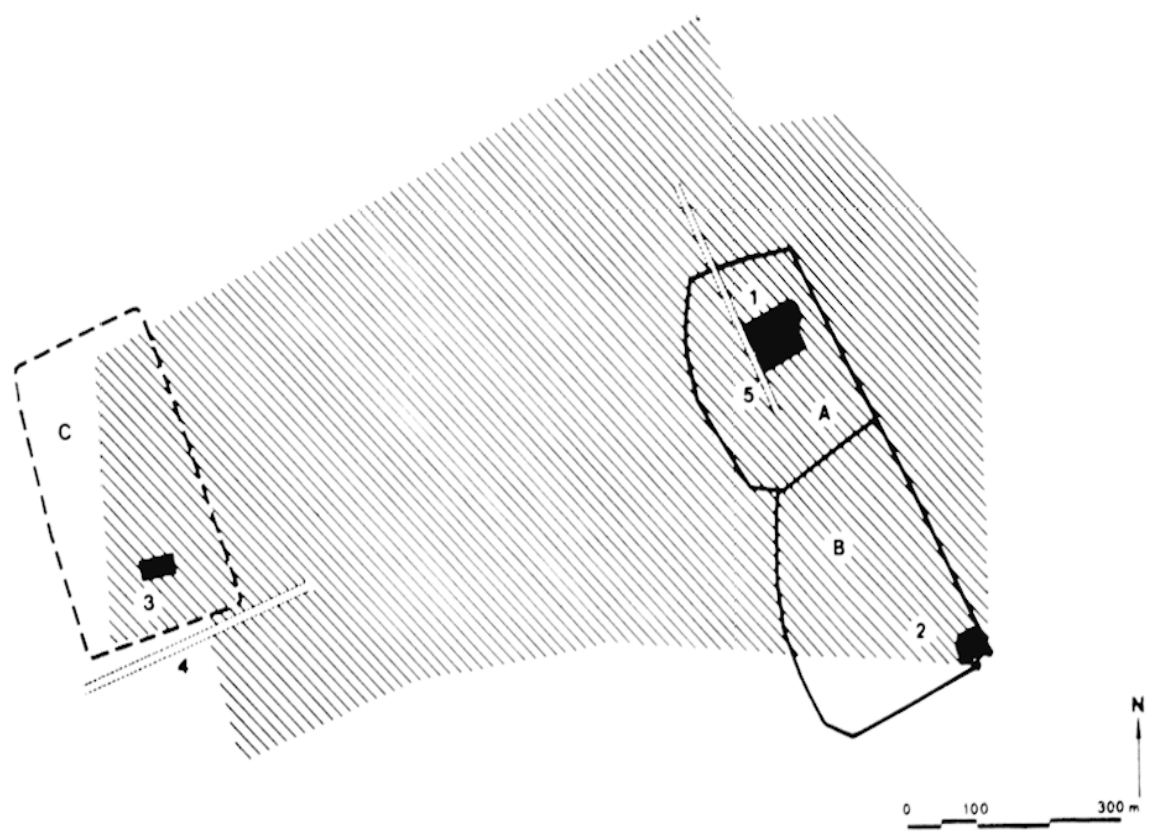

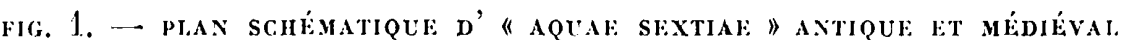
DEssix: S. ROVCOLE

lin hachures obliques, extension probable de l'habitat antique (à l'intérieur d'un rempart dont le tracé est très hỵpothétique). Fn A et B, bourg Saint-Sauveur et Ville comtale du Moyen $A g e$ : le tracé rectiligne du rempart est de ces deux noyaux urbains est à l'emplacement de l'actuelle rue Pierre-et-llarie-Curie (cf. fig. 13) : 1) groupe cathédral Saint-Sauveur; 2) palais comtal construit en partie sur la porte antique d'Italie; 4) et 5) decumanus et cardo maximus. Le site de la Cour de l'Archevêché est à l'est du groupe cathédral.

Comme le site avait connu en outre bien des transformations avant la dernière et radicale intervention des bulldozers, l'information recueillie est donc très inégale en quantité et en qualité selon les secteurs et selon les époques - autant de traits qui expliquent la difficulté que nous rencontrerons plus d'une fois à présenter une synthèse assurée. Ainsi, pour commencer, dans l'étude des plus anciens éléments reconnus par la fouille.

\section{Antiquité}

La présentation rapide d'un relevé précis de l'ensemble des vestiges antiques du site (fig. 2) fournit sans doute la meilleure introduction à l'étude de l'Antiquité. Ses lacunes donnent une image, d'ailleurs insuffisante, de nos ignorances (3), sa confusion en certains endroits trahit la complexité d'une longue histoire tandis que la trame régulière de l'ensemble montre l'existence d'un schéma d'urbanisme qu'il importe de relever pour guider notre enquête.

Les grandes taches grises des sols en béton dessinent au premier coup d'œil deux ensembles majeurs, d'ailleurs inégalement connus ou conservés : celui du sud (ou insula II) est très largement oblitéré par des constructions modernes bâties sur caves tandis que l'insula I, au nord, doit sa relative préservation à sa situation sous la Cour même de l'Archevêché (fig. 3). Deux rues de direction générale est-ouest (4) (ou decumanus) encadrent ce dernier ilot : l'aile nord du palais archiépiscopal masque largement la première tandis que la plus méridionale apparait dans toute son ampleur, même si elle est encombrée de constructions adventices; à l'est s'étend un autre espace ouvert de largeur indéterminée, sur la nature duquel nous aurons à nous interroger : rue (cardo?) ou dégagement?

Le carroyage ainsi défini ne sert pas seulement à fixer un cadre commode pour les descriptions, il révèle 


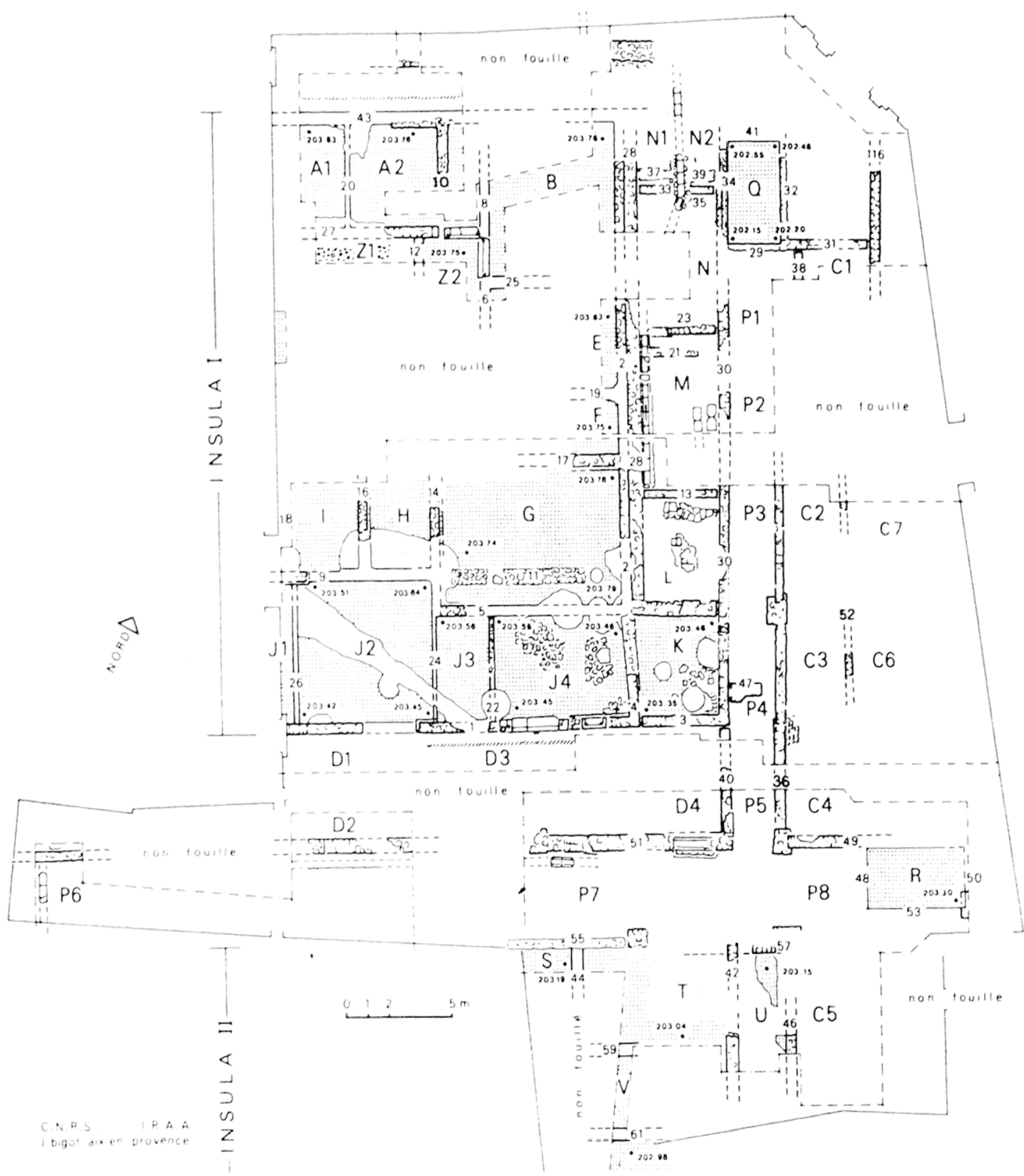

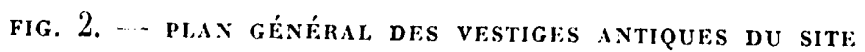

N. B. - Le mur de façade des écuries du xviri siècle, qui marquait jusqu'en 1984 la limite sud de la Cour de l'Archevêché était sensiblement à l'emplacement du mur 51. Relevé : I. R. A. A., Bureau d'Aix-en-Provence : P. Varène, J. Bigot J.-M. Gassend, J.-M. Joulain, avec l'aide de (;. Magdinier; mise au net : J. Bigot. 


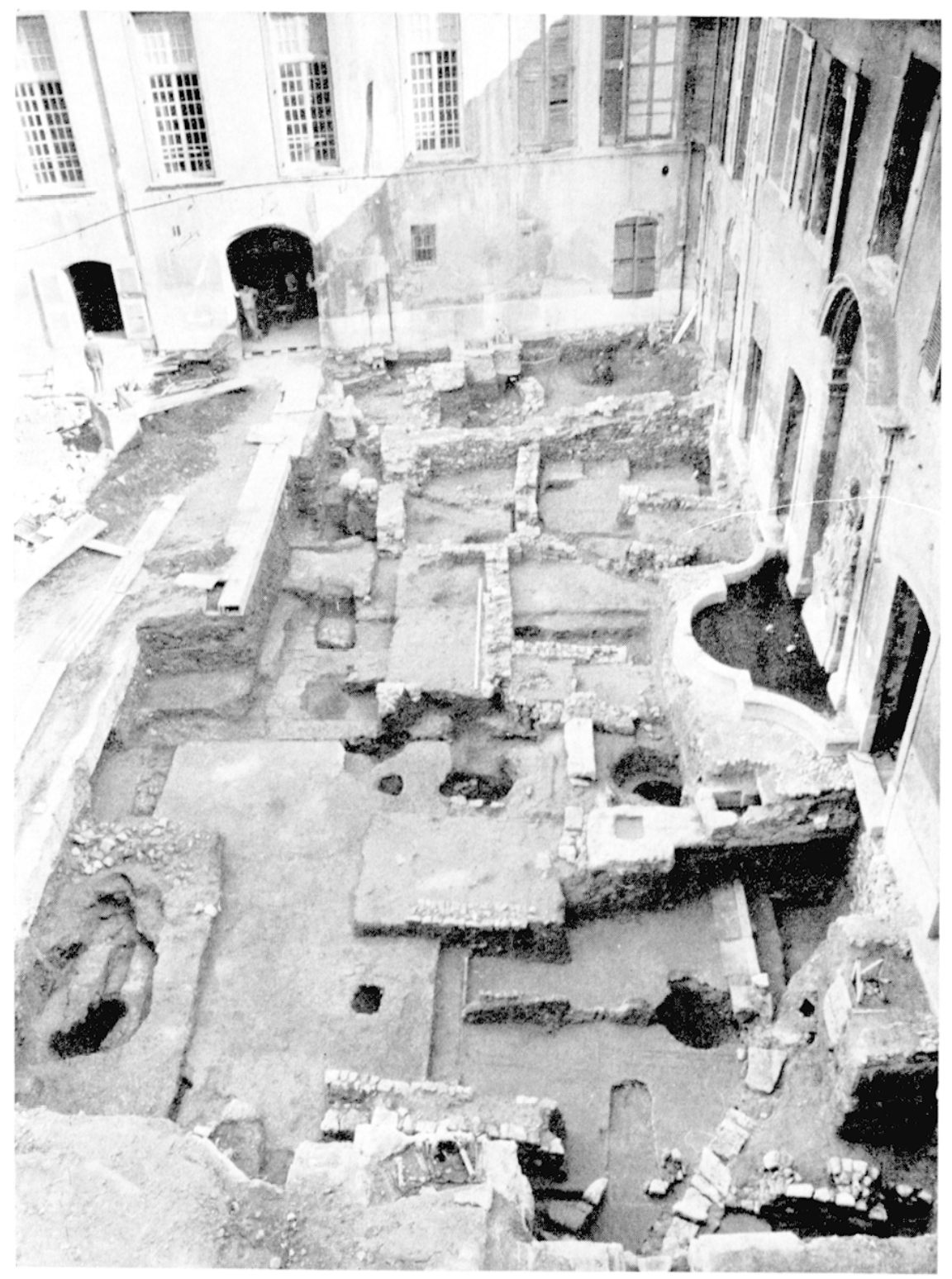

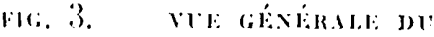

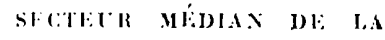
FOCHALE PRISE DE LOUEST 1 DROTTE, I.F MUR DES

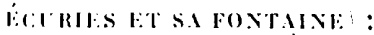
l. P.ARTI: MÉRIDIONAL: D) L. "

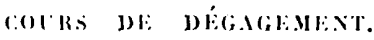

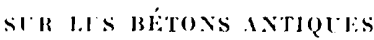
PFRFORISS PAR DIES SHOS,

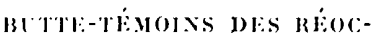
Crpatoxs MÉDIÉvaLES

aussi le plus amien plan d'urbanisme que la fouille ait permis à la fois de recomnâtere et de dater précisément, tout en montrant qüil avait sùrement existé au moins un itat antérienr d'occupation. C'est par la présentation de ces vestiges les plus anciens qu il ronvient de commencer, mêmr si noms ne pouvons guère les dater ou en présenter une restitution assurée.

J. - Le premer schéma d'urbinisus: je: sttr:

\section{a) Description}

LES "INSULAE 》 PRIMITIVFS

L'insula I présente des traces évidentes de transformations à la fois par les doublages ou collages de murs qui affectent tout son secteur oriental et par la présence à l'ouest de murs profondément dérasés que la fouille a retrouvés sous les bétons du demier état du bâtiment.

Ces murs sont au sud, dans la pièce (i, le nur 11 et au nord, en $A 2$, le mur 10, d'ailleurs lié au mur de façade de l'insula dont il ne subsiste plus qu'un témoin du parement sur la paroi d'une profonde tranchie de récupération. Ils ne sont conservés qu'en fondation, ce qui peut expliquer que leur largeur soit variable (respectivement $0^{\mathrm{m}} 70$ et $0^{\mathrm{m}} 60$ ) et ils sont composés de blocs mal dégrossis liaisonnés à l'argile. 


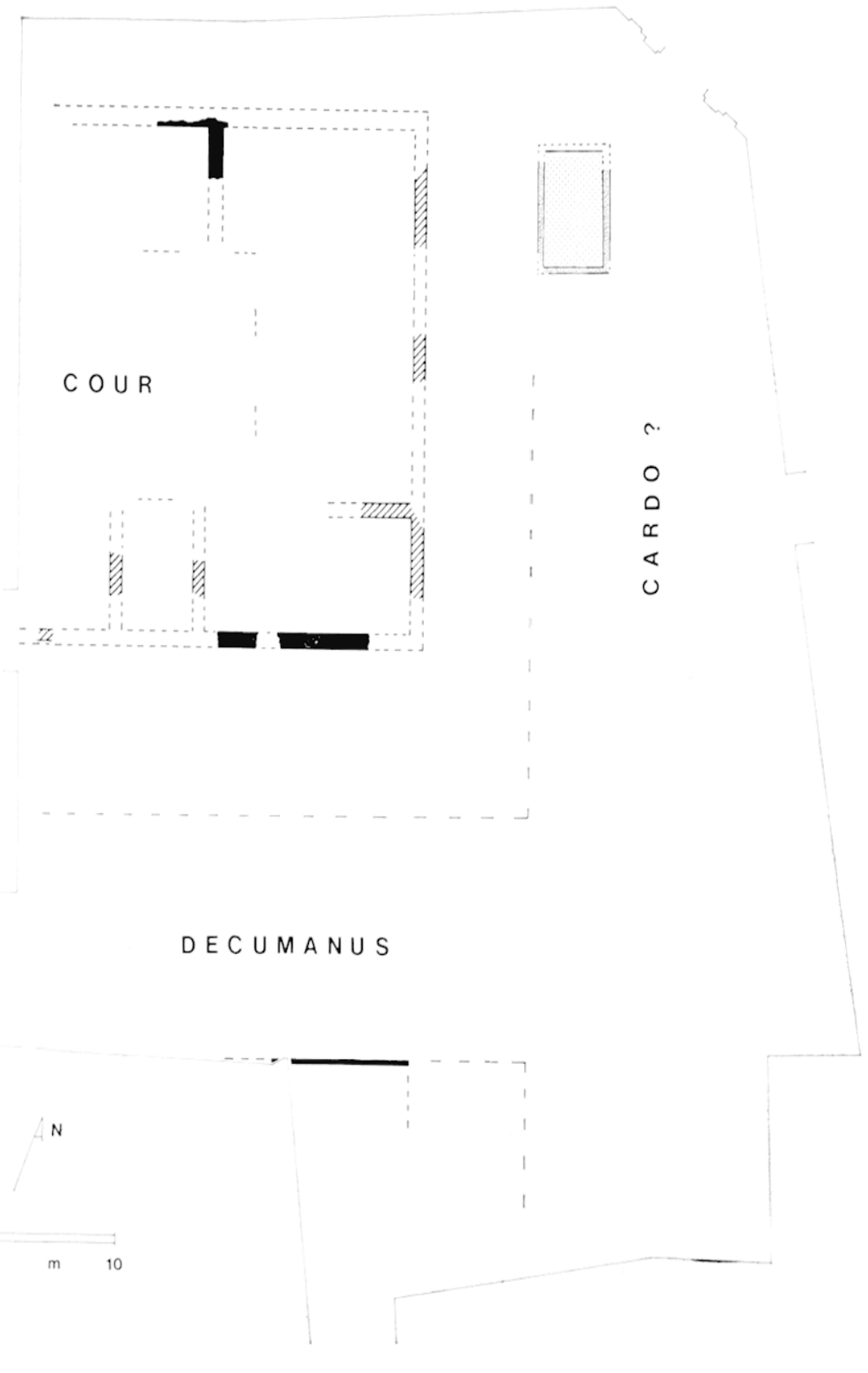

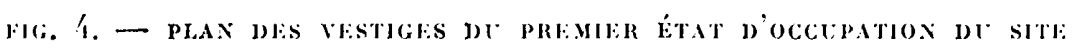

La même technique de construction est souvent employée dans tout le secteur occidental du bâtiment mais ne se retrouve jamais à l'est du mur 2, et très exceptionnellement au sud du mur 11 : a son extrémité occidentale, le mur 1 a bien un appareil liaisonné a l'argile, mais à l'est, notamment à proximité des deux seuils, un mortier assez pauvre a été employé. A considérer le schéma de la figure ', sur lequel les murs 10 et 11 ont été figurís en noir tandis que les autres murs liés à l'argile sont en hachures, l'impression prévaut done que l'insula achevée (dont le contour est matérialisé par un pointillé) est née de l'extension vers l'est et vers le sud d'un novau primitif plus réduit - mais dont la direction des murs maîtres a commandé toute l'évolution ultérieure de l'édifice.

On ne saurait être aussi affirmatif à propos de l'insula II, à la fois trop mal conservée et trop incomplètement reconnue; notons seulement que le tracé du mur de façade nord, tel que nous l'avons restitué, réunit des èléments fort différents : d'ouest en est, un massif de hiton hydraulique, 55, épais d'une quarantaine de centimetres, que nous avons reconnu sur une longueur de plus de 5 mètres et qui pourrait avoir servi à préserver de l'humidité les fondations de l'immeuble (fig. 5), puis deux puissants dés de fondation, sur lesquels nous reviendrons, infra, p. 221, entre lesquels prend place un élément de mur, 57, que nous avons seulement entrevu au-dessous d'un mur moderne dans la cour sud-est des communs du palais archi- 


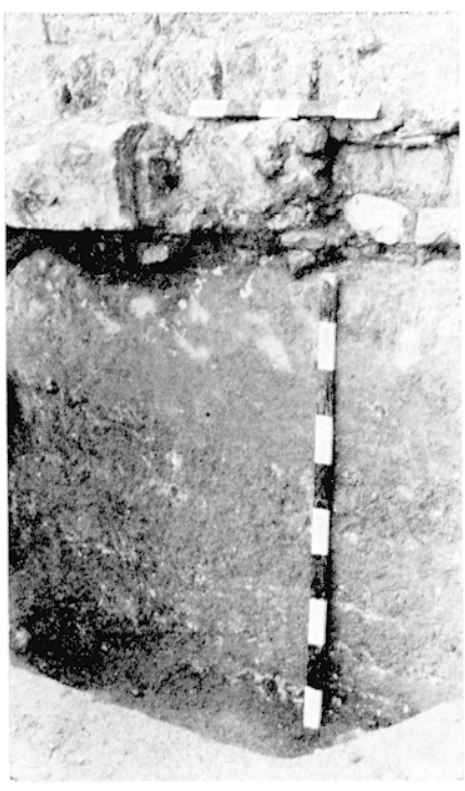

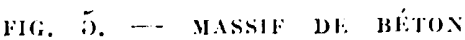
HYDRAT LIQT: ANTIQLF CONSERVI: FX FoNdohtox DU MCR DE FOND DES ĹCURIES 1) PI PALIS IRCHIÉPISCOPAL vU DC NORD-OUEST (C\%. AUSSI FIG. 72 ;

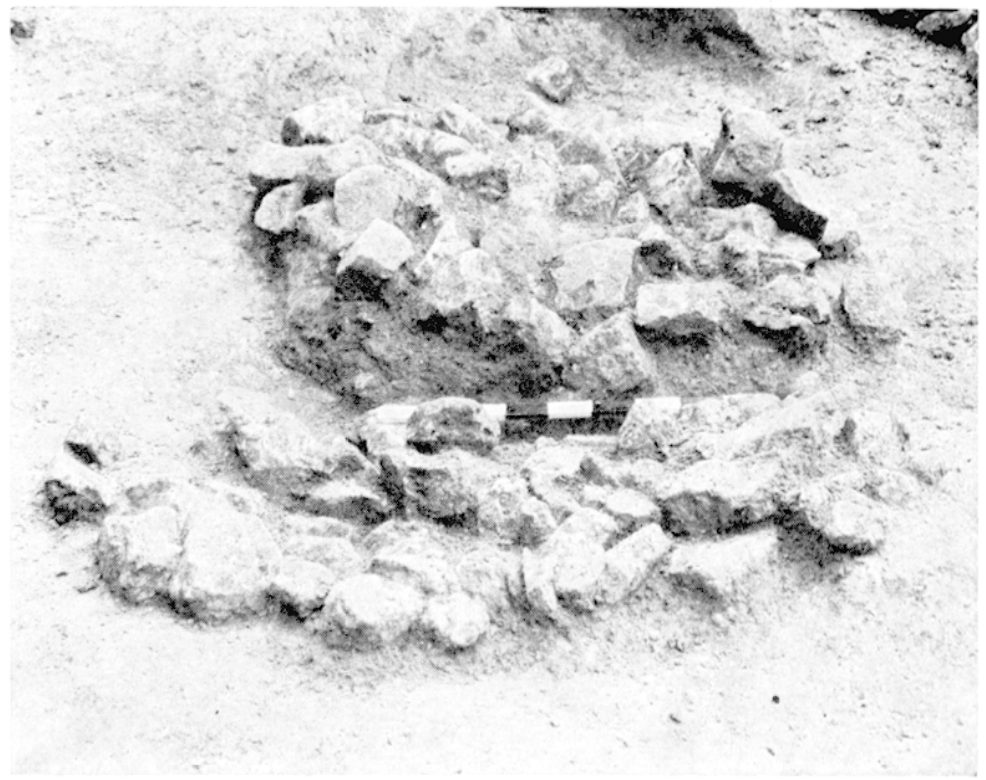

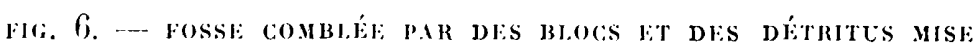

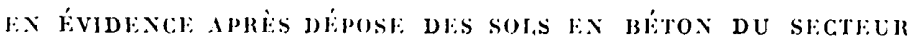

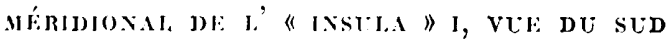

épiscopal (cf. fig. 2). L'hétérogénéité de ces constructions suggère que comme l'insula I, l'insula II est un bâtiment qui a connu une ou plusieurs extensions en direction de l'est et - hasard ou fait exprès - on notera que la césure entre les éléments de murs 55 et 57 se situe à l'alignement du mur 2 qui marque précisément dans l'insula I la limite entre noyau originel et parties ajoutees.

\section{LES ESPACES OLVERTS AI.ENTOUR DES " INSULAE"}

Entre les deux noyaux primitifs des insulae I et II tels qu'ils apparaissent à l'analyse (donc entre les murs 11 et 55 ), la fouille a retrouvé, tant sous les bétons tardifs de l'insula I que sous la chaussée en cailloutis du decumanus, des traces plus anciennes d'occupation, dont l'allure diffère sensiblement de part et d'autre du mur 1 qui sert de façade sur la rue à l'insula achevée.

Lintre les murs 1 et 11, la dépose des bétons a révélé l'existence de trois grandes fosses : les deux premières au centre el au sud-ouest de la pièce $\mathrm{J} 2$, la troisième au centre de la pièce $\mathrm{J} / \mathrm{z}$ qui abritait également dans l'angle sud-est un foyer aménagé à la cote $203,07 \mathrm{~m}$; ces fosses avaient élé comblées par des blocs et contenaient aussi quelques déchets animaux (fig. 6). Nulle part plus au nord, nous n'avons rencontré sous les bétons de semblables vestiges qui s'établisseut en outre à une cote sensiblement inférieure à la fois à celle des sols que l'on peut restituer dans l'insula primitive $(203,75 \mathrm{~m}$. au nord, $203,45 \mathrm{~m}$. au sud (5)?) et au niveau d'afileurement du sol vierge au sud du mur 1.

Au sud de ce mur en eflet, l'argile naturelle est conservée à la cote $203,17 \mathrm{~m}$. et forme comme une banquette large d'une soixantaine de centimètres qui paraît longer toute la façade de l'insula acheviée (cf. fig. 1'1 ) (6); là où il a été possible de le vérifier - essentiellıment en D3 - il semble que le sol naturel a en revanche été plus profondément aflouillé au sud de cette banquette, pour alteindre la cote $202,85 \mathrm{~m}$.

Au nord de l'insula 1 , la situation est assez semblable : une banquette d'argile est également conservée au long du mur 43 et le sol naturel a bien été profondément excavé au nord de cette banquette; mais surtout il a été fortement entaillé ici par une tranchée de direction est-ouest dont scule la paroi sud, d'ailleurs pourvue d'un parement maçonné médiocrement conservé, est visible sur la fouille, $2 \mathrm{~m}$. environ au nord du mur 43 , en lisière des fondations de la façade du palais archiépiscopal.

A l'est du mur 2 enfin, la situation est notablement différente selon les endroits. On distinguera pour les décrire trois zones qui correspondent sensiblement dans l'histoire ultérieure du site (et la coïncidence n'est peut-être pas fortuite) d'abord aux extensions orientales de l'insula I, ensuite au portique construit tardivement en façade de ce bâtiment (voir infra, § IV), au reste du secteur oriental de la fouille enfin.

Sous les extensions orientules de l' "insula » I, entre les murs 2 et 30 , la fouille a rencontré l'argile vierge à des cotes 


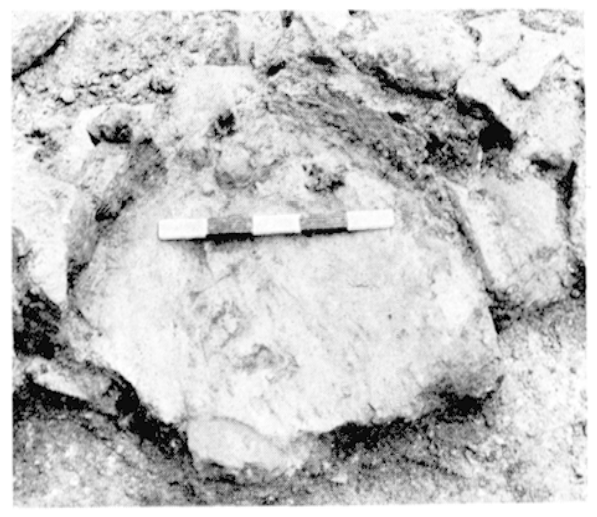

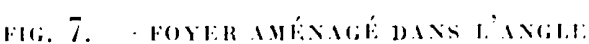

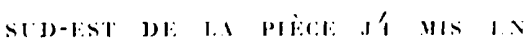

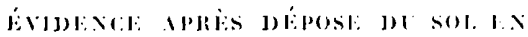

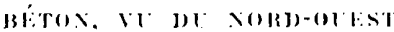

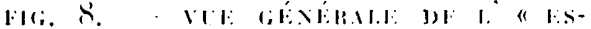

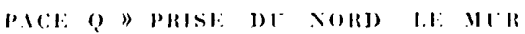

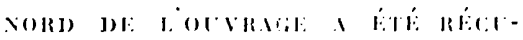

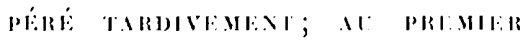

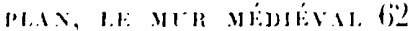

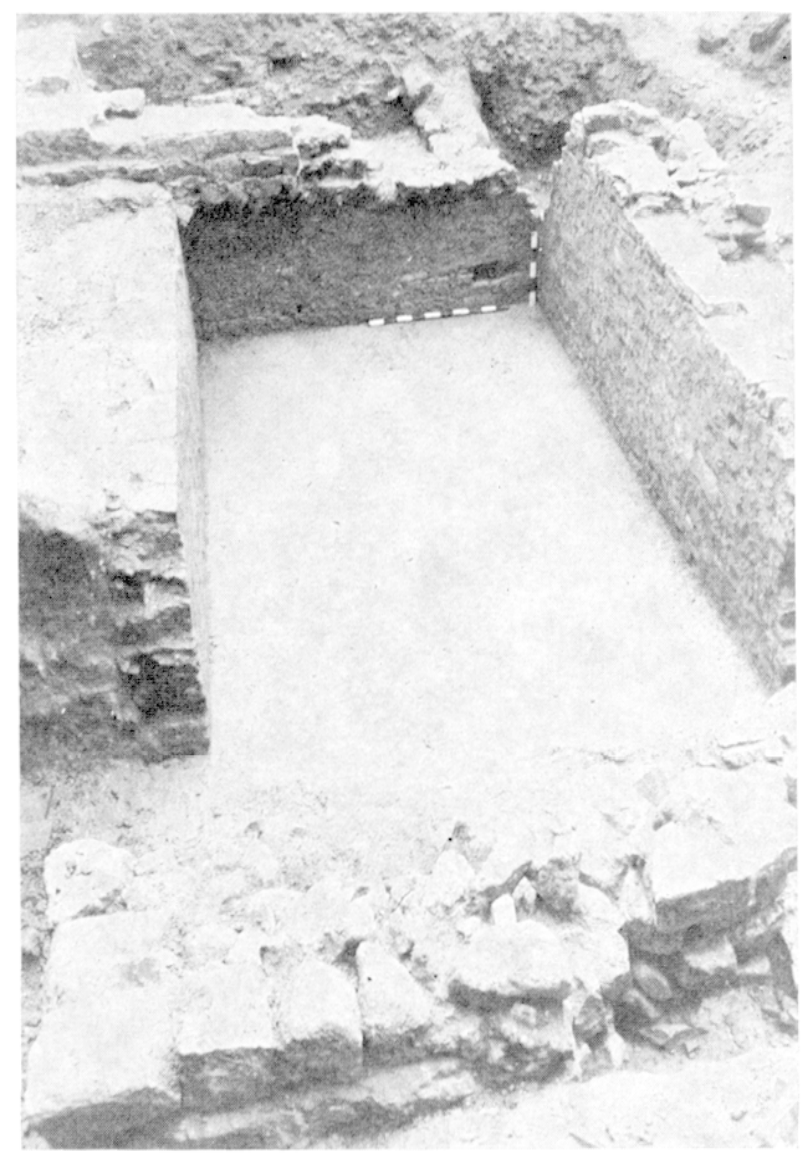

de plus en plus profondes en progressaut vers le sud : 203,55 m. dans la piece $\mathrm{N}, 203,15 \mathrm{~m}$. en I, 202,75 m. enfin dans la piece K. Si la difference de niveau entre $Y$ et l. peut s'expliquer par le pendage naturel d'un terrain en pente descendante du nord vers le sud, la cote étonnamment basse de li tient à un surcreusement; le sol de cet espace est d'ailleur's le seul à prisenter des traces d'une véritahle occupation comme an témoigne le niveau cendreux qui le recouvrait et qu'il faut sans doute mettre en relation avec le foyer aménagé de l'angle sud-est de la pièce : a un niveau encore inférieur, la situation est ici assez comparable à celle déjà rencontrée dans les pièces voisines, $\mathrm{J} 2$ et $\mathrm{J}$ 't.

Jans la bande de terrain comprise entre les murs 30 et 36, done dans l'ensemble des secteurs P1 à P5, la fouille n'a en revanche retrouvé sous les sols du portique en façadle de l'insula aucune trace d'un aménagement antérieur, mais elle a livré plus au nord, dans l'espace () un ouvrage qui est certainement ancien car le mur de façade de l'insula achevée repose sur son inur ouest (7).

Cet ouvrage avait reçu au cours de la fouille l'appellation de bassin à cause de sa profondeur et de la finition soignée des joints de ses parois, simples parements d'une grande fosse creusée dans le sol naturel. Comme il est possible de le vérifier par l'empreinte laissée par le nur 41 dont l'appareil a été récupéré, le maitre d'ouvre a commencé par recouvrir d'un béton de chaux le fond de cette fosse qui accuse d'ailleurs une forte pente du nord vers le sud (de 202,55 a 202,15 m.) puis il a élevé des murs latéraux dont l'épaisseur est variable $(30$ à $\mathbf{4} 0 \mathrm{~cm}$.) à cause du profil oblique de la paroi d'argile contre laquelle ils ont été dressés. Sur le côté sud, près de l'angle sud-ouest, à 10 centimètres environ au-dessus du niveau du fond se voit une petite ouverture de forme irrégulière d'ailleurs partiellement rebouchée (fig. 8). Si le sobriquet de chantier donné à l'ouvrage doit être pris au séricux, on pourrait songer à un dispositif de vidange qui n'aurait d'ailleurs janais èté nuis en service car l'argile naturelle est intacte derrière cette évacuation supposée.

Dans le reste du secteur oriental de la fouille, où la recherche a été très inégalement poussée, la situation enfin varie sensiblement selon les endroits. Alentour de l'espace Q, le sol vierge est conservé très haut $(203,25 \mathrm{~m}$. à l'est) tandis que dans les secteurs $\left(2, C^{2}\right.$, et $C 7$, sa configuration 'st voisine de celle que nous avons découverte au nord du mur 43 et au sud du mur 1 (donc devant les farades de l'insula I achevee) : au-dessous d'un sol en cailloutis comparable à celui du decumanus, l'argile vierge se rencontre à nouveau à des cotes profondes (jusqu'à 202,47 m.) mais sa surface est cette fois très inégale, dessinant des sortes d'alvéoles juxtaposés qui ont parfois pris d' $1 \mathrm{~m}^{2}$ de superficie.

\section{b) Restitution}

Par prodence et par sonci de simplification, nous avons représenté sur le schéma de la fig. '́ les seuls espaces bâtis qui appartiennent, probablement on certainement, au premier état de l'orcupation humaine sur le 


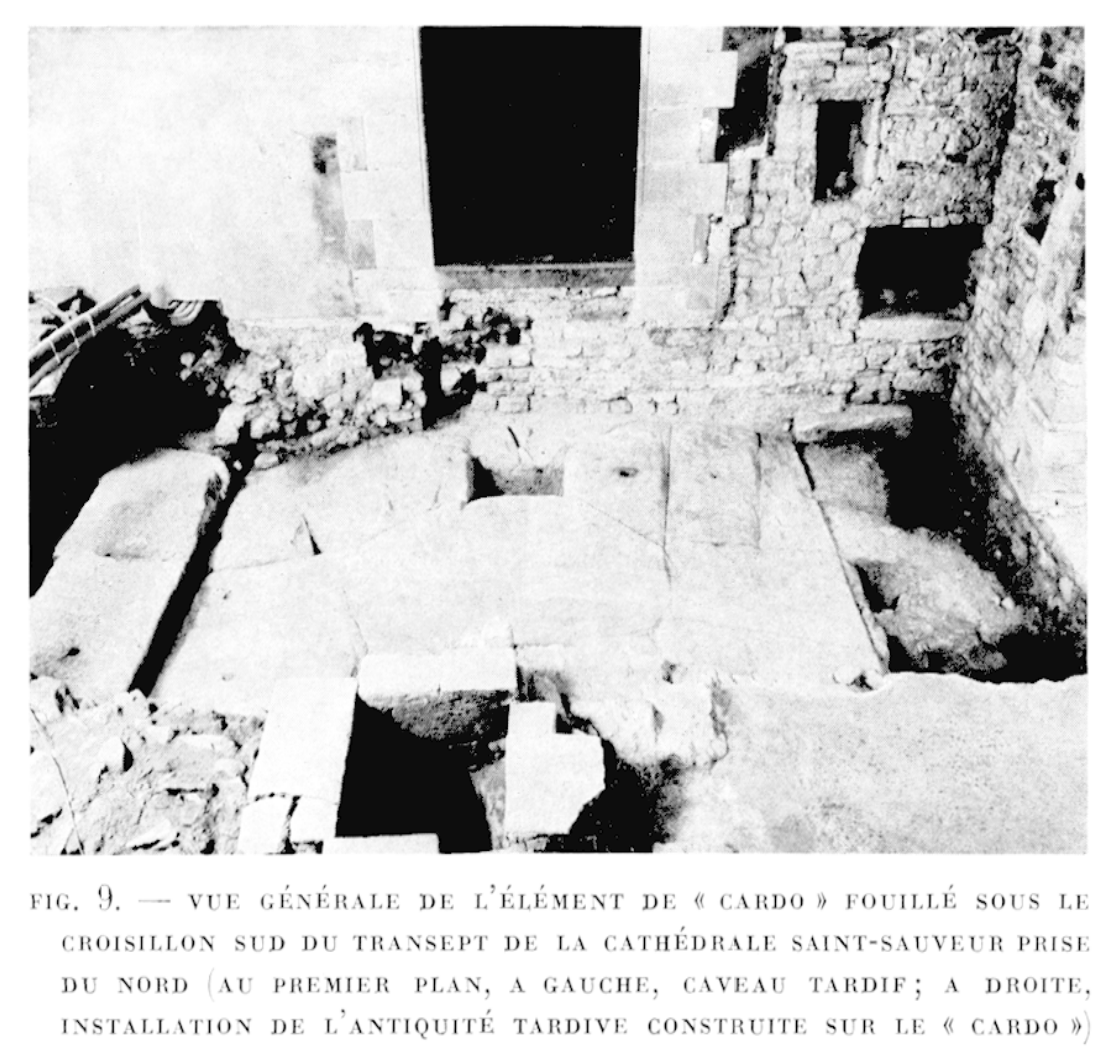

site : à coup sûr le noyau primitif de l'insula I et l'énigmatique ouvrage (Q; peut-être une partie de l'insula II. Alentour, la situation est différente dans les espaces situés à l'intérieur et à l'extéricur de l'emprise de l'insula I achevée matérialisée sur le schéma par un tracé en pointillé). Il s'agit, dans une restitution. de tenter de rendre compte de ces differences.

A l'extérieur du réseau de pointillés, nous avons surtout noté (outre l'espace (Q) les banquettes d'argile et le surcreusement régulier du sol naturel au long des murs 1 et 43 , ainsi que les alvéoles dessinés dans l'argile vierge en $(2, C$, et $C 7$. A considérer la profondeur générale de ces excavations et surtout le profil particulier qu'elles présentent à l'est, nous nous sommes demandés en cours de chantier si elles ne conservent pas l'empreinte de dalles massives analogues à celles du cardo que la fouille encore inédite de la cathédrale Saint-Sauveur a mises au jour sous le croisillon sud du transept gothique (fig. 9 ) tandis que la banquette d'argile qui longe les murs 1 et 43 aurait pu servir de support à un trottoir (8).

Nous serons plus prudents aujourd'hui pour présenter cette hypothèse, non seulement parce que la fouille n’a guère retrouvé d'éléments qui aient pu appartenir à un dallage éventuel 9 ) mais surtout parce que la restitution de chaussées pavées sur l'ensemble des surfaces considérées ne va pas sans difficulti : au nord et au sud de l'insula, nulle trace sûre d'alvéoles tandis qu'à l'est, la conservation de l'argile naturelle à une cote élevée à proximité de l'espace $Q$ interdit d'imaginer que si dallage il y a eu, il ait couvert l'intégralité des espaces C.. Il reste que ces surcreusements existent, et seulement sous des niveaux de cailloutis qui ont certainement servi a la circulation dans l'histoire ultérieure du site; aussi verrions-nous volontiers en eux les premiers témoins d'un aménagement de chaussées dont il faut peut-être renoncer à restituer précisément le faciès primitif. Le faciès, mais non toujours l'étendue et de ce point de vue, la situation est différente au sud et a l'est de l'insula I achevée.

Au sud, la distance entre les murs 1 et 55 est de $8 \mathrm{~m} 88 \ldots$ soit exactement 30 pieds. Il est satisfaisant de rencontrer un module simple, d'ailleurs employé dans d'autres villes romaines comme Fréjus (10) mais une telle largeur n'a jamais été relevée à Aix pour les rares tronçons des rues antiques qui ont été observés (11), sauf peutêtre dans un cas -.- celui de la voie d'Italie à son entrée en ville à travers la porte conservée jusqu'au xvme siècle dans les fortifications du palais des comtes de Provence ia l'emplacement du Palais de Justice actuel, au sud-est de la ville : ef. fig. 1 j. S'il faut en croire des témoignages anciens, la rue avait mesuré environ 9 mètres de large à cet endroit (12); mais il s'agit d'une voie majeure qui passe traditionnellement pour le cardo maximus d'Aquae Sextice (13). Faut-il s'autoriser de ce parallèle pour supposer que c'est le decumanus maximus qui a été reconnu au sud de la Cour de l'Archevêchè? ('e n'est pas impossible car cette portion de voie est sensiblement dans l'alignement 
de la rue de Célony qui peut avoir gardé dans la topographie moderne le souvenir d'une voie majeure de l'dntiquité 1 '); faute de preure plus explicite, largument reste pourtant fragile...

A l'est de linsula I achevée, en revanche, point de limite nette et l'on peut hésiter entre drux hypothèses : chaussé large ou chaussée etroite? Ia restitution d'une rue d'un module réduit peut s'appuyer sur l'existence d'un mur, 3L, qui a lardivement barre l'espace a l'est de l'insula I en repesaut d'ailleurs partiellement en fondation sur la paroi sud de lespaee (2 if. fig. 2- laxamen do l'appareil montre en effet que le mur butte aux deux extrémités contre d'autres constructions : a l'ouest, la fagade de l'insulu I achevée ; l'est, un mur, ll6, dont l'appareil est lic a la terre : faut-il restituer a cot endroit la façade doun autre immenble antique et imaginer que le mur 31 barre de toute sa longueur - - m $^{\mathrm{m}}$ i) - une voie romaine?

Comme la plupart des rues d'Aquae Sextiae ont une largeur de 6 a 7 mètres, lhypothese serait seduisante si le mur 116 s'apparentait plus nettement aux autres construetions antiques du site (15) et surtout si l'on avait

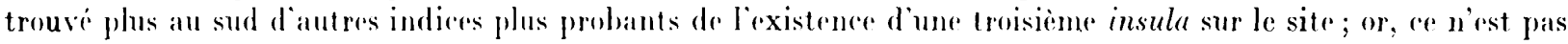
le cas. Il est vrai qu’a l’emplacement de l’édifier attendu, dans l'angle sud-est de la Cour, de profonds et vastes surereusements une grande fosse et des silos médievaux qui se sont d'ailleurs recoupés empêchent de reconnaître la situation antique; mais pourquoi res remaniements du .Ioyen Age auraient-ils fait disparaître ici toute trace deventuelles comstructions anterieures, alors que partout ailleurs, ils nobliterent jamais tout a fait la lecture des edifices antiques, s’ils la gènent considerahlement? Aussi croirions-nous plus volontiers a l'existence d'un espace ouvert large d'une quinzaine de mètes au moins, boulevard plutòt que rue, à l'est de loinsula I - ce qui pourrait d'ailleurs expliquer que loun ait très lit empieté sur ce boulevard ? pour construire l'ouvrage Q.

Le mot de boulevard evoque un ouvrage militaire et de fait, en essayant de restituer le trace est du rempart d'-Lque Sextiae, qui est sans doute la portion la phus mal conmue dine enecinte qui nous échappe presque totalement, l'historiographie aixoise a souvent replace la muraille antique à proximite immédiate, sous la rue Pierre-etMarie-Curie, en supposant que l'enceinte médievale -. - la recta linea du xine siècle -.. reprenait à cet endroit un tracé plus ancien, d'ailleurs situe dans le prolongement d'une portion de mur retrouvée plus au nord, au parking Pasteur (ex-lire du Chapitre et que son inventeur, Rouard, rattachait aux fortifications romaines 116 . Il serait aventureux de tirer des maigres indies fournis par la fouille un argument décisif en faveur de cette interprétation mais il est sûr que l'aspert de l'espare ouvert à l'est du site se comprendrait mieux s'il était situé en lisière d'un réseau urbain, sinon d'un rempart 17 ).

Quel que soit le crédit a acrorder au détail de ces restitutions, il reste que l’on croit entrevoir, dès le premier état d'occupation du site, l'existence d'un réseau de circulation qui est pourtant à l'écart, à l'est comme au sud, du noyau primitif de l'insula I, tout en ayant des directions qui sont celles des murs maîtres de ce premier ensemble. Ia similitude des axes montre quäa la difference de tant d'autres villes antiques, Aix n’a pas commu de transformations profondes de son schéma diurbanisme mais le hiatus constaté entre surfaces bâties et niveaux de circulation semble le signe d'un repentir dailleurs difficile à interpreter surement.

Faut-il en effet reconnaître un statut autonome au noyau primitif de l'insula I, donc supposer qu'il correspond à un premier îlot urbain qui aurait ité entouré de larges dégagements avant qu un plan régulateur ne dessine alentour les chaussées que nous avons restituées et rende ainsi possible le lotissement de marges orientales (L a $\mathrm{N}$ et méridionales ( $J$ et $\mathrm{K}$ ) qui relevaient jusqu'alors du domaine public? Ou doit-on supposer au rontraire que c'est le réseau de circulation qui est premier, donc que les propriétaires du terrain correspondant à l'ensemble de l'insula I achevée navaient d'abord bâti qu'une partie de la surface qui leur était dévolue, réservant pour un second temps l'aménagement des lranges est et sud de leur lopin?

L'étude des premières traces d'oceupation dans les espaces $J$ et $K$ ne permet guère de trancher entre ces deux interpretations ar les foyers et les niveaux cendreux que nous avons déerits peuvent être rattachés indifféremment au chantier de construction des extensions du noyau primitif de l'insula ou au contraire à une première phase d'utilisation, en marge d'un îlot originel deja construit : ils auraient alors pris place sur un espace ouvert en lisière de rue, voire au sein d'un enclos - - disparu - dont les murs 1, 3 et 30 anraient ultérieurement repris le tracé.

Cette restitution d'un bâtiment entouré d'annexes ouvertes iou d'un enclos) a toutes chances, notons-le, de décrire une situation qui s'est effectivement rencontrée dès lors que furent aménagiés les chaussées : comprendrait-on sans cela le profil identique que présente le sol naturel en lisière des murs 1 et 13 ou encore la construction de la paroi ouest de l'ourrage (Q dans l'exact alignement du futur mur 30 -.- tous traits qui supposent que le plan même de l'insula I achevér était déjà inscrit sur le site quand furent réalisés ces différents aménagements? La seule incertitude est donc de savoir si ce plan, sûrement ancien, est aussi le plan primitif des lieux ; mais convientil de trop renvoyer du mal connu a linconnu, de distinguer plusieurs phases de transformations, d'ailleurs impossibles à imaginer et à dater précisement, au sein d'un ensemble primitif qui a été aussi sommairement reconnu? Mieux vaut sans doute sen tenir a la vision plus svnthétique que suggère le schéma de la figure 4. 


\section{c) Datation.}

Rien ne permet de dater sûrement les plus anciens vestiges du sitr. Dans l’insula I. l'argile qui lie les murs faits de blocs mal équarris de lî̀lot primitif est stérile et du premier citat des chaussées, nous ne commaissons que des négatifs; quant aux comblements des fosses des pièces J ou celui de l'ourrage Q, ils temoignent de transformations à mettre en relation avee les extensions de l'insula : nous les etudierons done an \$ II.

A examiner la céramique et les séric's monétaires les plus anciennes trouvies comme éléments résiduels dans l'ensemble des remblais (qui peuvent provenir pour partie do terrassements affectues sur le site mêmej, il

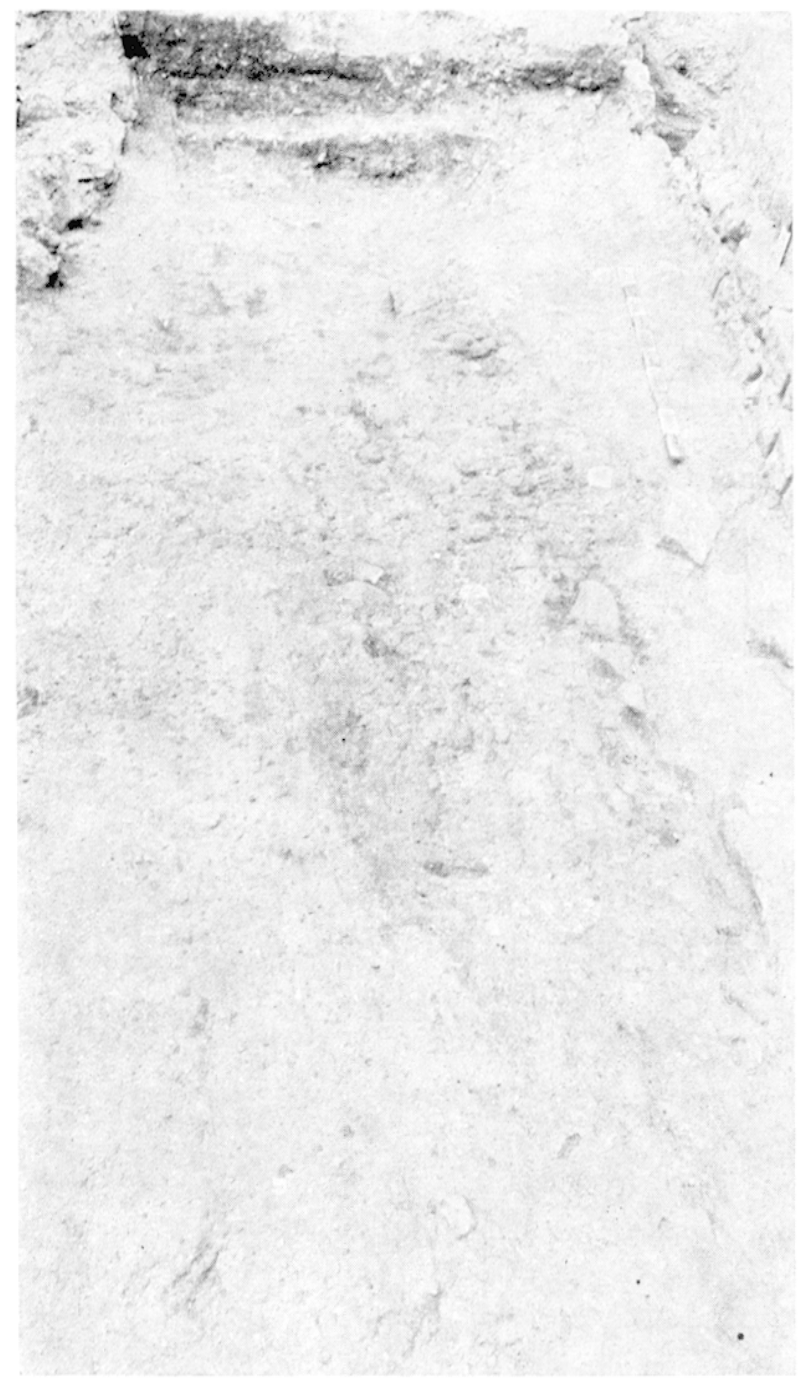

FIG. 10. - SOL FN CAHILOTIS SUR LE "DFCUMANUS" VUE GÉXÉRALE: DE IOOULST ne semble pas co tout ras que la première orcupation des lieux soit très ancienne. ()uatre monnaies $n^{0} 1$ a 1 de lappendie numismatique en annexe: et quelques fragments de ceramique seulement sont en effet sùrẹ-

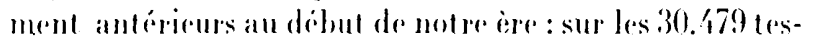
sons antiques recueillis is soit 23.018 dans les courhes antiques of 7.161 dans les niveaux médiévaux, 22 dont 12 provellant dhorizons medievaux/ sont de ceramique ampanienne soit a peine 0,07 00 o du total. Le chiffer est d'aulant plus éloquent que même en incluant la céramigue arctine qui a éte produite et diflusere autour du

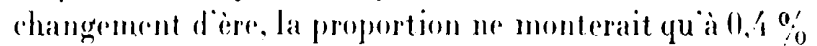
1.30 tessons an tout, dont 23 issus de couches medirvales!. Le trait sacoorde trop ave lensemble des trouvailles archémogiques faites jusqu ied a lix qui nous rapportent, dans le meilleur des cas, à l'époque augustéene pour que la prudence ne commande jas de rattacher sensiblement à la même periode les premicrs vestiges de la lour do l'Arehevêehé.

Sur cre site aussi, les restes de la première fondation de Sextius Calvinus nous exhappent done totalement. Ja remarque peut surperendre quand nous venons d'evoquer pourtant la possible proximite d'un rempart - ou an moins d'une limite urbaine -... a l'est de la fouille : faut-il supposer que nous sommes en lisière diune ville qui ne se serait developpere que tress lentement? Ou est-ce une invitation a abandonner delinitivement lhypothèse qui place sous le hourg Saint-Sauveur le prétendu castrum du dernier quart du 11 e siecle a vant notre ère (19!? Mieux vaut laisser ces questions ouvertes a elles sont un stimulant a poursuivere la recherche.

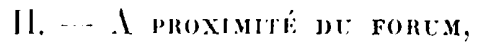

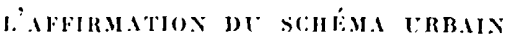

Lutant les datations sont floues aux origines, autant les indications se multiplient au premier siècle, traduisant une évolution rapide de l'habitat et des rues. L'occasion est bonne de pratiquer une première coupe chronologique vers la fin du siècle, en un temps marqué non seulement par l'achèvement de l'urbanisation du secteur mais aussi par la transformation de tout un quartier qui abrite désormais l'ensemble monumental du forum. Avant de présenter cette évolution majeure de la topographie urbaine, il convient d'en rester au site et d'étudier le domaine public et l'espace bâti de l'insula I.

a) Le domaine public.

\section{Descriptron.}

Alentour du périmètre de l'insula I achevée, il a partout été possible de repérer un niveau de circulation vers la cote $203,40 / 203,25$ m. (20). La reconnaissance de ce niveau a cependant été inégale et surtout elle a livré des sols d'aspect assez 


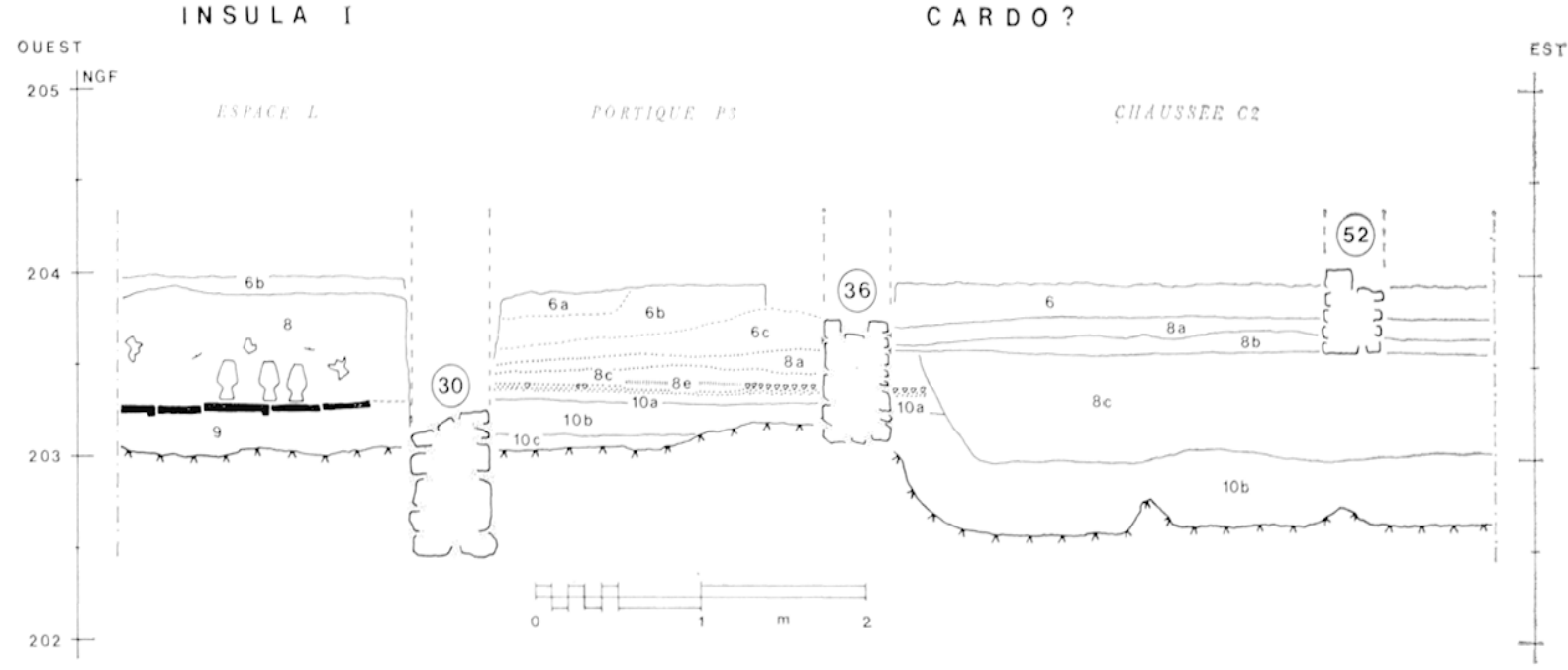

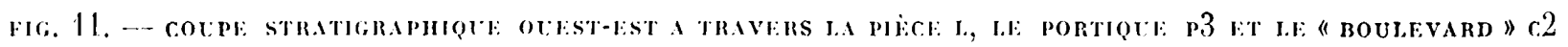

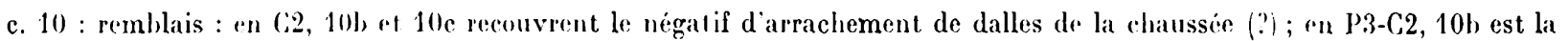
couche d'apprèt pour la mise en place du sol en cailloutis 10a; c. 9 (espace 1.) : remblai supportant un sol couvert de tuiles; c. 8 : dans la pirce I., remblai au-dessus du sol de tuiles contenant des vases entiers (cf. fig. 15); sous le portique P3 : 8e : foyer entaillant le cailloutis 10 et contenant une monnaie de Domitien (annexe numismatique, no 9); $8 \mathrm{c}$ : niveau de circulation chaulé à la cote $203,47 \mathrm{~m}$. (cf. tableau VI) ; 8 a : niveau de circulation chaulé à la cote $203,58 \mathrm{~m}$. (cf. tableau V'II); sur le "boulevard " (;2 (cf. tableau V) : $8 \mathrm{c}$ : importante fosse contenant essentiellement des amphores (cf. aussi annexe numismatique $n^{\circ 5}$;, 7,12 et 13) recouverte par un remblai argileux 8b; 8a : niveau de circulation en terre battue; c. 6 (tous espaces) : remblais de l'Antiquité tardive (cf. tableau VIII).

différent selon les endroits : empierrement de cailloutis en 1'3-Ci2 et surtout P5 et 1)4 où l'aire dégagíe atteint plusieurs mètres carrís (fig. 10) ; nappes hétonnées assez grossières dans les différents espaces 1 ; lambeaux de sols en terre battue dans les zones qui ont connu de fréquents remaniements. Ja diversité d'aspect peut être grande; il faut pourtant rattacher ces divers témoins à un seul et même sol comme le montre l'étude de l'espace P'3 où cailloutis et béton de chaux sont en continuité, le bèton s'appuyant d'ailleurs contre le mur 30 qui marque la limite orientale de l'insula I achevie (fig. 11).

Ce sol nous retiendra done non sculement parce qu'il répond au premier niveau de circulation connu sur des chaussées que nous n'avons pu que restituer hypothétiquement jusqu'à présent mais aussi parce que sa datation fournit un terminus ante quem non assez lâche pour l'achèvement d'une insula I qu'il entoure de toutes parts. Parler d'un terminus assez lâche n'est pas une simple clause de style : le bétonnage grossier de l'espace P't passe en eflet au-dessus de l'arase d'un mur, 47, qui a lui-même été appuyé contre le mur 30 de l'insula ct sans pouvoir interpréter autrement celte construction aux fondations puissantes (elles atteignent la cote $202,50 \mathrm{~m}$.), nous retiendrons surtout que sa scule présence atleste d'une histoire plus complexe que celle qu'il nous est donni de retracer.

\section{Datatiox.}

Le tableau suivant fournit la liste des espaces où nous avons pu ì la fois reconnaitre le sol des chaussées alentour de l'insula I et fouillar les remblais sous-jacents, ou couches 10 (21) et surtout il donne la nomenclature de la ceramique issue de ces remblais.

De son étude, on retiendra pour l'anecdote l'abondance relative pour le site) des céramiques campanienne et arétine et essentiellement la présence de céramiques qui fournissent de bons éléments de datation : 8 des 63 tessons de sigillée du sud de la Craule appartiennent à des formes produites et diffusées après l'an 60 de notre ère et l'on comprend bien leur association avec trois fragments d'orlo annerito et un bord de patina cenerognola qui se rencontrent également dès le milieu du ${ }^{\text {er }}$ siècle. Les années $60 / 70$ fourniraient donc un terminus ante quem non plausible pour la formation du sol des chaussées et l'on peut signaler comme une curieuse coüncidence la présence d'une pièce de monnaie de peu pestérieure elle date de 85) dans les cendres d'un petit foyer qui a entaillé (mais non perforé) le sol en béton de chaux dans l'espace $P 3$ (no 9 de l'appendice numismatique). 


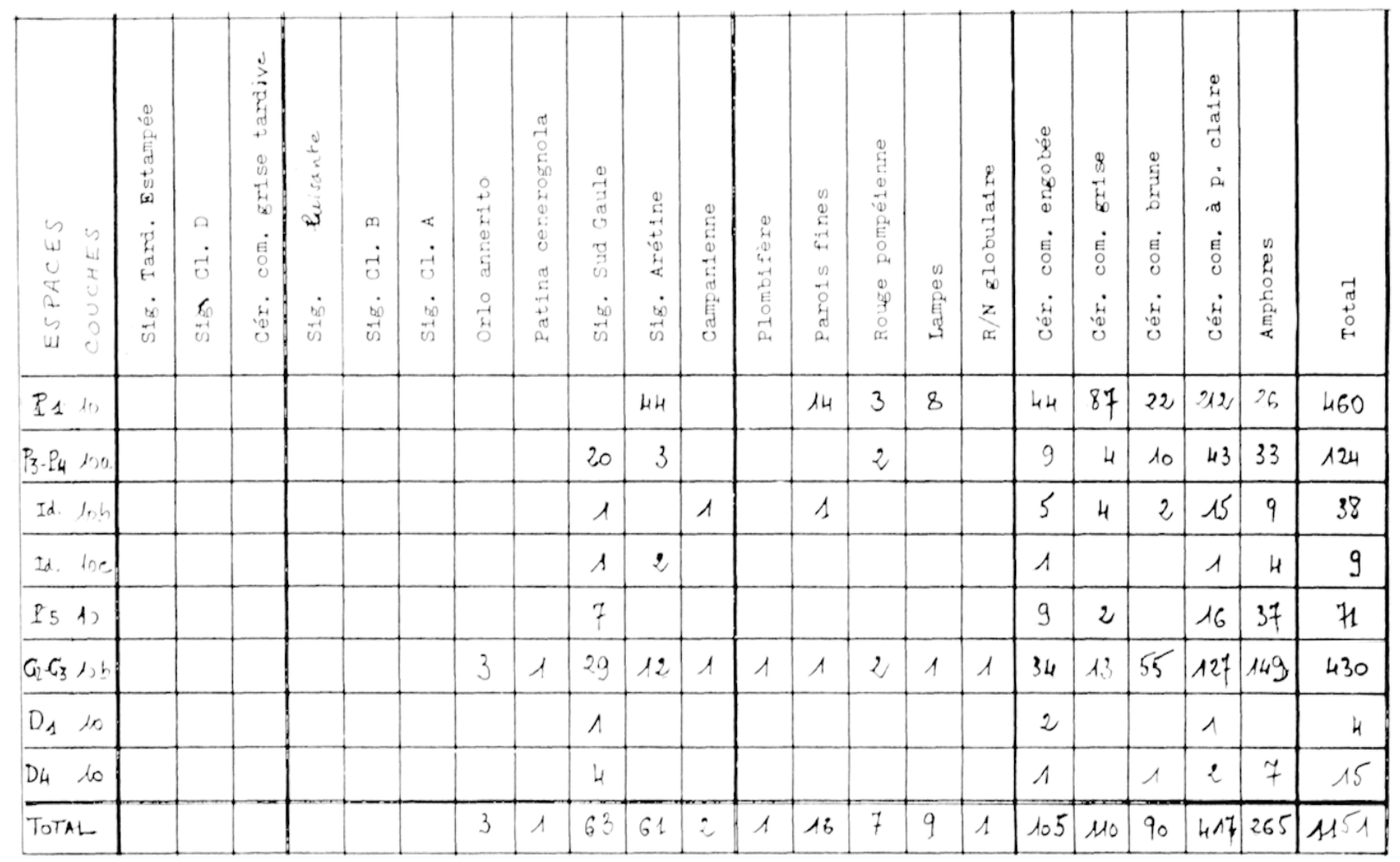

Tableav I. Nomenclature du matériel céramique provenant des couches placées au-dessous du niveau de circulation $203,10 \mathrm{~m}$. sur les chaussées.

\section{b) L'insula 1 .}

\section{Description.}

Les sols des chaussées cernent désormais les contours d'une insulı I agrandie, mais nous sommes peu au clair des conditions de cet agrandissement : résulte-t-il d'une ou plusieurs campagnes de travaux? Pour essayer de le deviner, nous réserverons notre altention à la scule pièce $\mathrm{K}$, exceptionnelle par sa complexité.

Si les deux murs est et sud de cette pièce, 30 et 3, sont bien liés, le mur 3 en revanche a été appuyé contre l'angle formé par le mur occidental, ' 4 , et le mur 1 qui sert désormais de façade à l'insula sur le decumanus, ce qui invite à distinguer au moins deux phases de construction (ou deux chantiers distincts) : une extension du bàtiment vers le sud, suivie d'un agrandissement vers l'est. Cet agrandissenent a été marqué aussi par la construction du mur 28 qui double lo mur 2, ancienne limite orientale du noyau originel de l'insula; mais ce mur 28 est plus long que le mur primitif contre lequel il a été appuyé (le mur 2 s'arrêtail originellement à hauteur du mur 11) sans pourtant traverser toute l'insula dans le sens de la largeur : autant que les médiocres conditions de conservation de l'angle nord-ouest de la pièce permettent de le vérifier (22), il semble en effet qu'il a été appuyé contre le mur 7.

Le trait est d'autant plus remarquable que le mur 7 est à l'alignement d'un autre mur, 5, qui ne fait pas partie non plus du noyau primitif de l'insula mais appartient à une transformation interne majeure de tout le secteur méridional de l'édifice : il a été élevé après arasement du mur 11, pour agrandir la pièce G' et sa construction suppose une importante modification des couverts. Coïncidence ou fait exprès que cet alignement des murs $j$ et 7 ? $\Lambda$ cause des récupérations médiévales qui ont fait disparaître le mur 5 à proximité de la pièce $K$, il est impossible de le savoir : sans doute le seul élément conservé du mur 5 a-t-il une facture plus soignée que le mur 7 mais l'indice est-il suffisant pour conclure qu'il y a là deux murs distincts, d'autant que le fond de la tranchée de fondation est à mème cote dans les deux cas $(202,85 \mathrm{~m}$.)?

liême si l'on voulait rapporter à un seul ouvrage les murs 5 et 7 , faudrait-il rattacher cet ouvrage à une transformation, parmi d'autres, de l'insula ou supposer au contraire, qu'il répond à l'une des tranches d'un unique chantier d'extension de tout l'édifice? Comment savoir quand les indices pour une datation absolue font défaut et que le monument est si mal conservé que l'interprétation de ses restaurations est aléatoire : ainsi, la construction du mur 28 avait-elle pour seul but de fournir un appui aux couverts de tout le nouveau secteur oriental de l'insula ou ee mur anx fondations en blocage servait-il aussi à conforter l'appareil liaisonné à l'argile du mur 2?

Vicux vaut laisser ces questions ouvertes et faire retour sur la pièce $K$ pour noter que ses parois ont reçu un revètement d'enduit peint qui peut appartenir à la période qui nous intéresse pour l'instant : cet enduit a en effet íté masqué et protégé à la fois par le sol en béton de la dernière période de l'édifice. Le fond blanc est rehaussé de fines lignes rouges : un trait horizontal court au long des parois, une trentaine de centimètres au-dessus du sol tandis que des verticales dessinent 


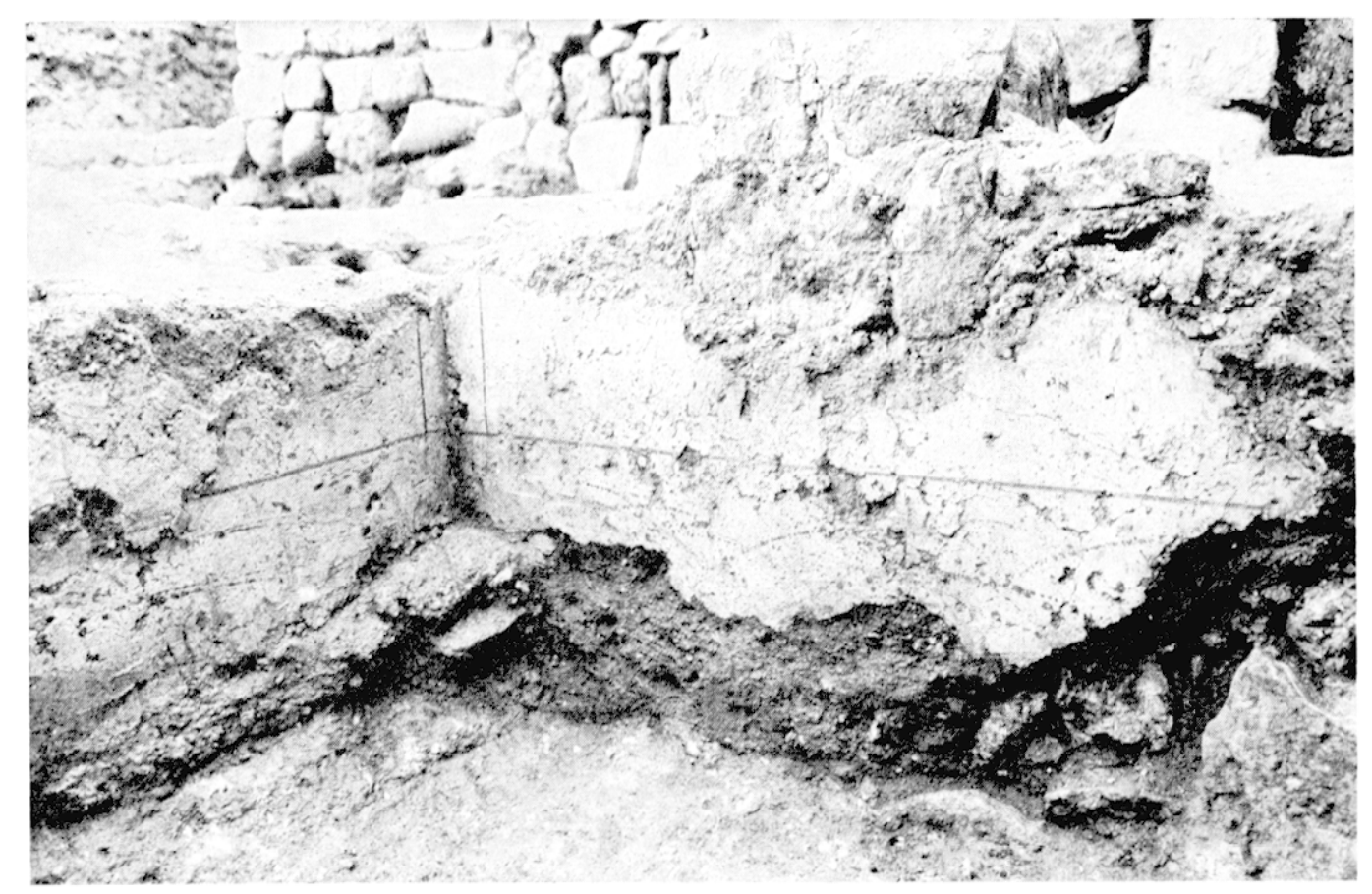

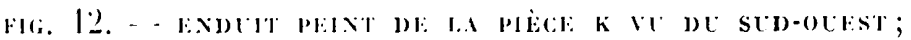

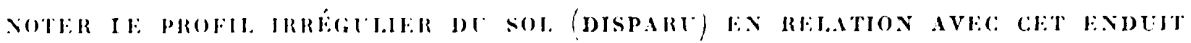

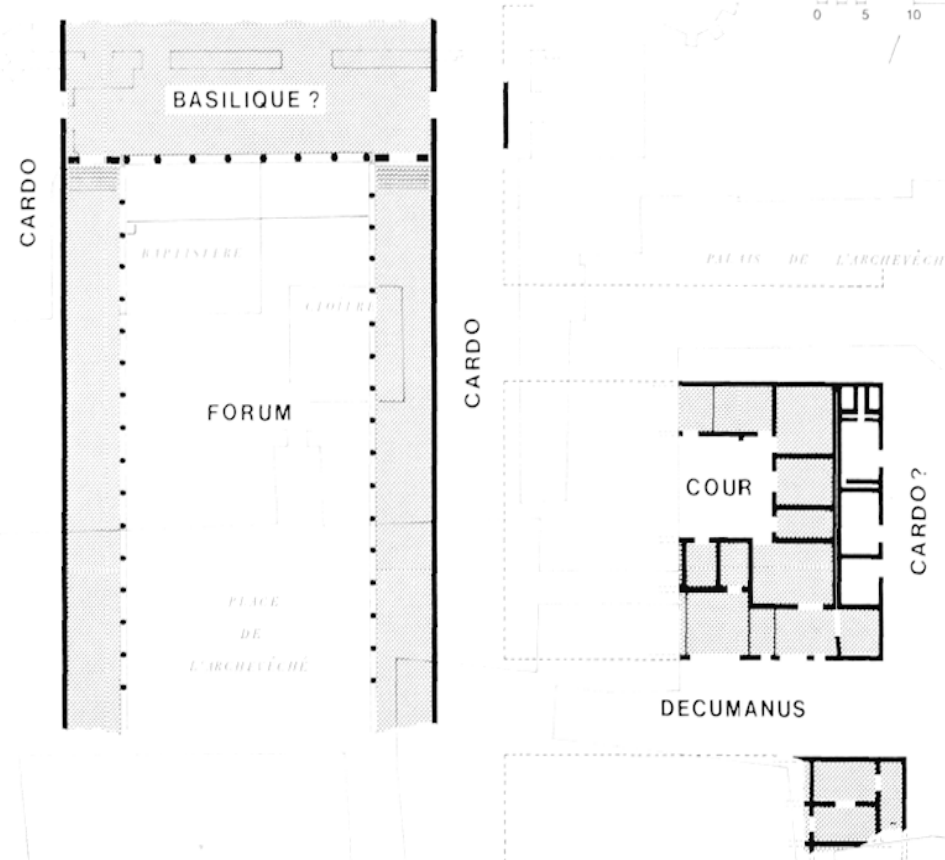

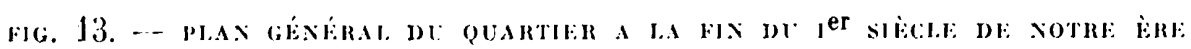


autant de petits panneaux; d'amples courbes de couleur rouge dont l'extrémité se résout en un simple mouchetis tombé du pinceau décorent d'autre part la bande inférieure (fig. 12). Le has de l'enduit dessine le profil, d'ailleurs assez irrégulier, d'un sol placé à un niveau très inférieur à celui des rues voisines et probablement aussi du reste de l'insula (202,95 m.) (23) : pourquoi avoir placé dans une position aussi déprimie une pièce que son décor relativement soigné (pour le site) signale pourtant à l'attention?

\section{Restrutrios.}

Liexamen de l'enduit peint conservé sous les bétons de la pièce $K$ prouve que le bâtiment a connu d'autres transformations que les extensions majeures que nous avons considérées jusqu'ici et une etude serrée livrerait d'autres rexemples encore : ainsi, toujours dans le même espace, le mur '́ a été tronqué, puis refait sans doute au moment de la pose du sol en béton du dernier état (21) et cette réfection pourrait expliquer pour partie le plan irrégulior, trapezoüdal, de la pièce 25 ). C'est assez pour montrer qu'il est impossible de restituer très préciś́ment la distribution intérieure d'un bâtiment que nous ne connaissons vraiment que dans son état achevé fig. 22).

Nous nous sommes pourtant résolus à représenter cet état sur le plan à grande échelle de la figure 13 parce qu il sagissait avec ce document de donner avant tout une représentation idéale du quartier vers la fin du er siècle de notre ère; mais de l'insula I à cette date, nous ne possédons en toute certitude que l'enveloppe. Fancore est-elle pour partie hypothétique à l'est car le mur 30 n'a pas été reconnu sur toute sa longueur; il est assez conservé pourtant pour que sa restitution et celle de l'angle nord-est du bâtiment soient tenues pour assurées.

On notera seulement que le mur 30 présente un aspect différent selon les endroits : dans tout le secteur méridional, il s'agit d'une construetion fondéc soigneusement et profondément a la cote 202, 行 m. ; a hauteur de l'ouvage () en revanche, la fouille n'a livré qu'un médiocre collage de murs, dû peut-être à une restauration ou plus simplement au fait que le mur est appuyé à ret endroit sur la paroi oceidentale du bassin isupra, p. 201) qui offrait wne fondation toutr prête, plus solide et plus profonde encore qu'ailleurs. L'extension de l'insula suppose d'ailleurs, a l'évidence, la désaffectation de louvrage (Q, qui est suffisamment documentée par la fouille : le comblement du bassin eontenait en abondance déchets de taille et rehuts de construetion, qui peuvent provenir de limportant chantier ouvert à proximite et la céramique associée peut fournir un nouvel indice pour la datation des extensions de linsula I.

\section{Datation.}

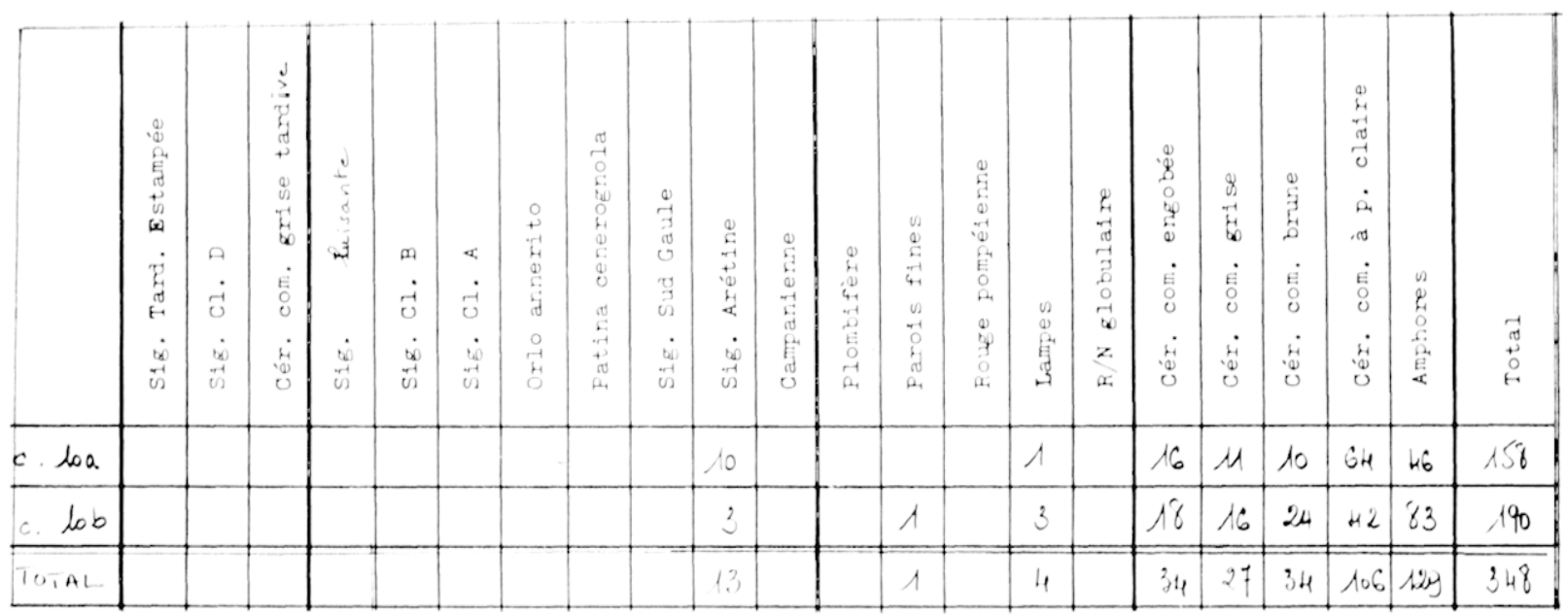

Tabisau II. Nomenclature du matériel céramique provenant du comblement de l'ouvrage Q.

Le tableau ci-dessus donne la nomenclature de la céramique recueillie dans le comblement de l'ouvrage Q. Les éléments de datation sont fournis à la fois par les productions arétines qui se rattarhent aux services II ct III de Haltern tandis que le service I est totalement absent) et par les formes de la céramique commune - - autant d'indices pour placer le remblaiement après les années 20 de notre ère.

A rapprocher cette datation de celle fournie par l'étude des remblais sous les chaussées qui entourent de toutes parts l'insula I après 60-70\%, un intervalle d'un demi-siècle environ est ouvert, au sein duquel l'extension du bâtiment a toute chance d'avoir été réalisée. N'oublions pas cependant que pour borner cet intervalle, l'examen de la céramique ne peut fournir que des terminus ante quem nôn et pour plus de sûreté, nous retiendrons que 


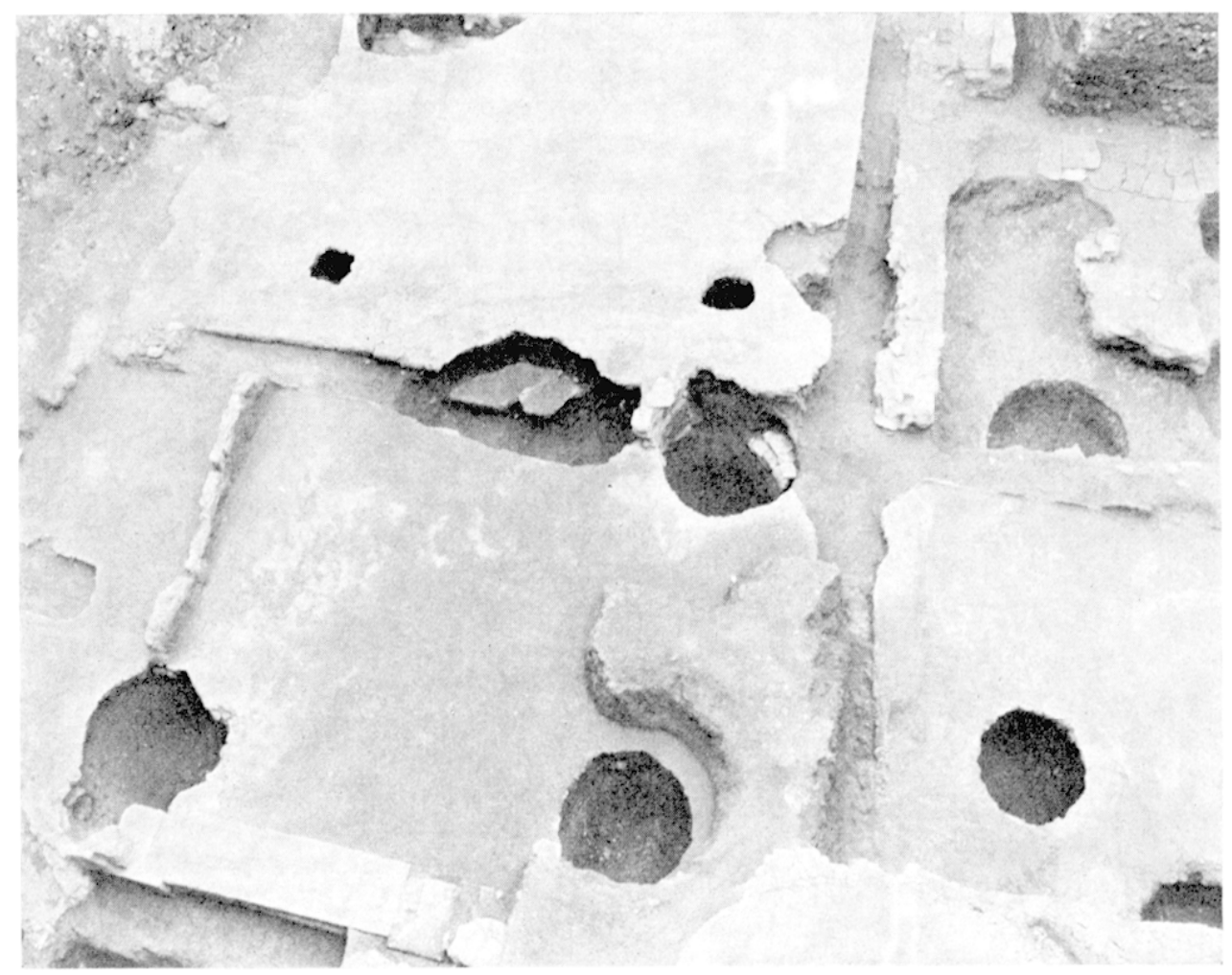

FIG. 14. - SOI. FN BÉTON DU SECTEUR MÉRIDIONAI. DE I. "INSLIA " I VUS DU SUD

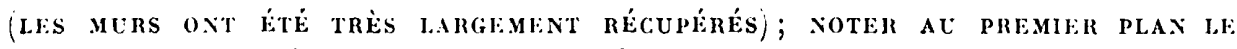

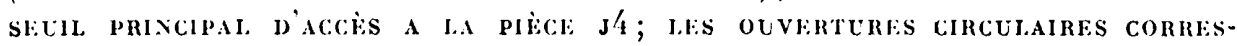

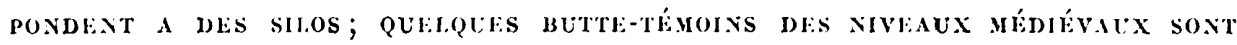
ENCORE CONSERUÉES

l'ensemble des transformations du secteur ont sûrement été achevées avant la fin du ${ }^{\text {er }}$ siècle, au moment même où l'ensemble du quartier prenait un visage nouveau.

\section{c) L'urbanisme réglé de la fin du Ier siècle.}

Pour apprécier l'urbanisme dans son ensemble, il est indispensable de rapprocher les données de la fouille de celles fournies par les recherches récentes menées sous la place des Martyrs-de-la-Résistance fex. place de l'Archevêché) et le groupe cathédral Saint-Sauveur 26\%. Le secteur de l'insula I fouillé dans la Cour de l'Archeveché apparaît alors comme la moitié orientale d'un îlot urbain situé immédiatement à l'ouest d'un ensemble monumental sûrement achevé lui aussi avant la lin du $\mathrm{I}^{\text {er }}$ siècle de notre ère et qui compte au moins une place bordée de portiques et un grand édifice public -... le forum d'lix et ses dépendances selon toute probabilite (fig. 13).

D'est en oucst, l'insula mesure $39^{\mathrm{m}}$;0 et cette distance se retrouve dans l'ensemble voisin du forum qui est inscrit entre deux rues de direction nord/sud précisément séparées par ce même intervalle de $39 \mathrm{~m} 50$. Si l'on ajoute que dans l'insula I, la proportion entre la longueur et la largeur est régie par un module simple $(39 \mathrm{~m} 50 \times 25 \mathrm{~m} 75$, soit environ 1,j, l'hypothèse qu'il ait pu exister au corur de la ville antique un urbanisme réglé par un plan strict se présente immédiatement à l'esprit. L'historiographie aixoise a cependant trop été prodigue - -. et récemment encore $(27)-$ en restitutions aventureuses pour que nous nous proposions une quelconque cxtrapolation à partir d'une fouille dont l'extension demeure trop réduite par rapport à l'étendue de l'agglomiration romaine.

D'ailleurs, sur le site même, le module rencontré à la fois au forum et dans l'insula I ne vaut sûrement que pour ces deux exemples comme le montre un simple regard jeté sur les vestiges de l'insula II : le mur est de ce bâtiment, 10, est nettement à l'est du mur 30 qui marque la limite orientale de l'îlot nord. Il serait sans doute possible de biaiser avec la difficulté en rappelant que l'insula II a sûrement connu, comme l'insula I, bien des transformations (28) et qu'elle compte un mur, 12 , qui est exactement à l'alignement du mur de façade de l'insula septentrionale (cf. fig. 2); placer à cet endroit, vers la fin du ${ }^{\mathrm{er}}$ siècle, la limite d'un édifice qui aurait été ultérieurement étendu en direction de l'est comme fut étendue aussi, nous le verrons, l'insula I (29j) serait assurément 
séduisant - mais gratuit : la présence de eaves modernes dans ce secteur a interdit en effet toute fouille de vérification.

Admettrait-on cette restitution qui rend aux îlots leur régularité que le réseau de circulation alentour resterait fort inégal, tant par la largeur des chaussées que pour la qualité de leur revêtenıent : 6 m85 pour les rues dallées qui longent le forum; $8 \mathrm{~m} 88$ pour le decumanus sud et une quinzaine de mètres au moins pour l'espace ouvert à l'est du site dont le sol est en cailloutis. Nous avons cru pouvoir expliquer ces différences supra, p. 203, en restituant à l'est un boulevard ?) et entre les deux insulae le decumanus principal d'a quae Sextiae; mais pour que ces restitutions soient plus que de simples hypothèses, il faudrait pouvoir produire un rempart à l'est et, au nord, un decumanus d'un module plus réduit ; or la fouille ne permet sûrement ni l'un ni l'autre 30 . Pour ajouter à la difficulté, le revêtement médiocre du decumanus sud surprend s'il s'applique bien à l'une des grandes artères d'une ville où d'autres rues sont dallées : a-t-on voulu iou pu) donner un aspect monumental aux seuls abords immédiats du fortum?

Toutes ces difficultés existent bien et leur arcumulation doit inciter à rester prudent dans les restitutions; il n'empêche : la seule répétition d'un module identique dans l'îlot I et le forum voisin montre suffisamment qưil y a bien eu, au moins dans ce secteur d'tix, une volonté consciente de créer un urbanisme réglé dont nous avions seulement entrevu les premiers linéaments en étudiant le plus ancien état de l'oecupation du site. A partir de la fin du ${ }^{\text {er }}$ siècle au moins, il faut done croire à l'existence dans le quartier d'un plan régulier - mais sans grandeur : comme celui des rues secondaires, le module des insulae est médiocre, comparé à celui qui a été utilisé dans la plupart des autres villes romaines (31). La même impression de médiocrité ressort de l'étude précise des îlots urbains eux-mêmes.

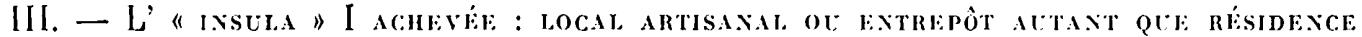

Les conditions offertes à la recherche ne laissent connaître passablement qu'une seule des insulae du site, l'insula I; au moins la fouille permet-elle de dater assez précisément l'état achevé dans lequel elle nous est parvenue. Renvoyant l'étude des transformations contemporaines ou postérieures de la chaussée au paragraphe suivant, nous nous arrêterons donc sur elle: ce sera l'occasion de pratiquer une seconde coupe chronologique dans l'évolution de tout le secteur.

\section{a) Description.}

De l'insula I, la fouille ne livre guère -- et encore partiellement -..- que le plan; aussi la description commencera-t-elle par les éléments les mieux conservés : les sols.

- Les sols.

LES SOLS EN BÉTON.

L'ensemble des pièces méridionales et tout le secteur occidental du bâtiment ont des sols en bíton, d'aspect d'ailleurs légèrement différent selon les endroits.

Fn G, II et I comme dans toutes les salles méridionales, il s'agit d'un béton de facture soignée, dans lequel la chaux l'emporte largement cependant sur les fragments de tuileau (fig. 14) : épais d'une dizaine de centimètres, il repose sur un remblai par l'intermédliaire d'un hérisson de pierres d'une quinzaine de centimètres d'épaisseur. le sol accuse partout des pentes régulières, en direction de la rue pour les pièces sud, du nord-ouest pour la pièce ( 3 ; la dénivellation dípasse parfois 20 centimitres entre les extrémités d'une même salle et les cotes moyennes sont plus élev'ées au nord (autour de 203,75 m.) qu'au sud $(203,45 \mathrm{~m}$.) ; à signaler enfin que dans la pièce $I$, un second sol en béton, dont il ne subsiste plus qu'un lambeau, a été placé sur le tapis primitif : trace d'une réfection impossible à dater.

Au centre, en Z, lE et $\mathrm{F}$, où la reconnaissance a été très sporadique, on notera surtout la différence marquée d'aspect entre le sol de ZZ, très proche des sols méridionaux et celui de Z1, fait d'un cailloutis serré scellé dans le béton - presque une calade da type de celle qui revêtait la Cour de l'A rehevêché avant l'intervention des bulldozers : il faut sans doute attribuer cette diffirrence de traitement à l'existence à cet endroit de deux secteurs distincts séparés par un mur, 12, que nous avons entrevu au printemps 198', à la faveur d'une excavation de reconnaissance que les services municipaux d'Aix-enl'rovence avaient ouverte préalablement au chantier; mais nous ne pouvons en dire plus.

Au nord enfin, dans les pièces $\$ 1$ et $\$ 2$ qui seules ont été víritablement fouillées, la conservation du sol est trèg médiocre, surtout par comparaison avec les pièces méridionales dont le revêtement apparait comme intact lien qu'il soit resté en service fort longtemps, comme le montrera la suite de cette étude : est-ce précisément parce que ce secteur a connu une histoire différente.

Les SOLS DF la partif onifitale.

Dans l'ensemble du secteur oriental (à l'exclusion de la pièce $K$ ), nous n'avons retrouvé nulle part des sols analogues 
par leur qualité a cenx qui viennent d'ètre décrits; la situation est cependant différente dans les trois espaces qui ont été individualisés.

Le sol de la pièce $L$, au sud, a presque entièrement été détruit par des remaniements très divers : fosse de l'Antiquité lardive, silos médiévaux, implanlation, puis récupération de murs tardifs. Seule une banquette large de moins d'un mètre a ele partiellement conservie an nord; elle a liver une straligraphie a la fois complexe et originale.

In remblat dune vingtaine de rentimitres d'epaisseur recouve l'argile virere ot sert de support, a la cole $2033,35 \mathrm{~m}$. a un sol constitur pour partic par de la terre baltue et pour partie par des tuiles brisenes posies a plat (cf. fig. 11): un temoin d'un premier chat d'occupation ou un sol de travail? Lin faverur de la seconde hypothese, il faul noter que des tessons d'un meme facies tres particulier se rencontrent a la fois au-dessous et aurdessus do re sol, dans un second remblai 32 .

L'essentirl du matérid céramique de ce dernier remblai apparticut en eflet a une senle fanille de céramique commune a pàte claire (33), d'une typologie originale (aucun parallele coumu sur les sites de refirence) : on remarque surtont des vases fermes, sans anses, al l'blal de fragments ou même entiers, posés a plat sur le sol de tuiles (fig. 15) aux côtés de petits couvereles munis de boutons de préhension pinces au doigt et d'un pot à deux auses de facture identique (fig. 16). Trois des vases sont intacts, trois autres aisément reconstituables tandis

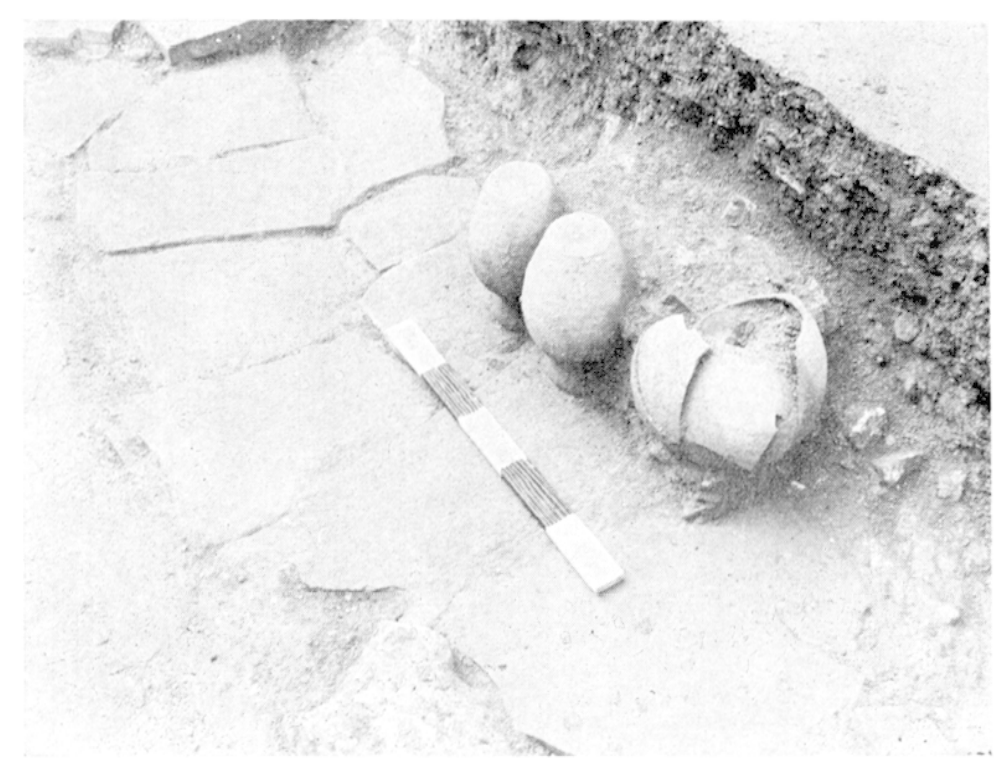

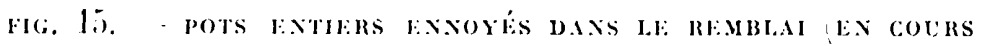

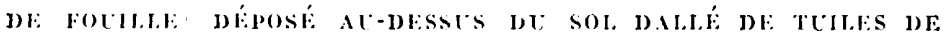

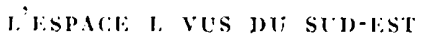
que le reste n'autorise que des remontages partiels. Si l'on ajoute que l'argile, d'un jaune très clair, est peu cuite et que quatre des pots sont voilés, l'ensemble s'apparente plus à des rebuts de cuisson qu'à la production commercialisable d'un atelier local.

La foulle attentive que nous avons pu consacrer a ce secteur fait ainsi connaittre l'existence d'une officine de potier aixois que nous imaginerions volontiers proche du site (des cales de four ont d'ailleurs été retrouvées en différentes zones dans les remblais du reste de l'insula : of. fig. 21). La période d'activite de l'atelier est cependant plus difficile à préciser. Pour l'établir, on ne saurait se fier aux seuls enseignenents tirés de la fouille du remblai : comme le montre en effet le tableau suivant, le seul élément de datation tient ici à la présence d'un unique tesson de céramique sigillée claire $A$, qui fournit comme terminus ante quem non less années 80-90 de notre ère.

\begin{tabular}{|c|c|c|c|c|c|c|c|c|c|c|c|c|c|c|c|c|c|c|c|c|c|c|}
\hline & 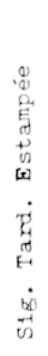 & $\begin{array}{l}\text { म } \\
\text { मे } \\
\text { मी } \\
\text { के }\end{array}$ & 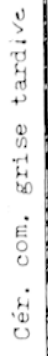 & 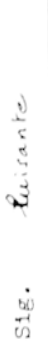 & $\begin{array}{l}\text { मे } \\
\dot{u} \\
\dot{0} \\
\vec{n}\end{array}$ & से & 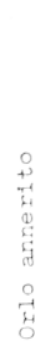 & 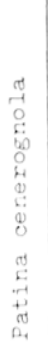 & 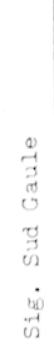 & 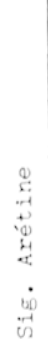 & 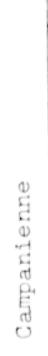 & 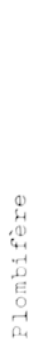 & 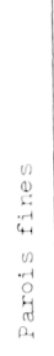 & 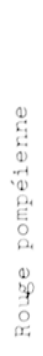 & 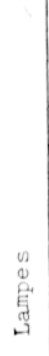 & 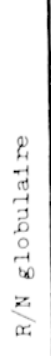 & $\begin{array}{l}\mathscr{0} \\
0 \\
0 \\
0 \\
0 \\
0 \\
\dot{E} \\
0 \\
0 \\
\dot{0} \\
\dot{0} \\
0\end{array}$ & $\begin{array}{l}\mathscr{8} \\
\overrightarrow{4} \\
\dot{6} \\
\dot{E} \\
0 \\
0 \\
\dot{0} \\
\dot{g} \\
\dot{g}\end{array}$ & 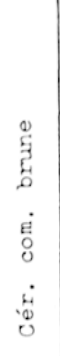 & 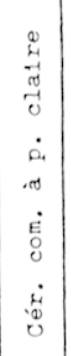 & 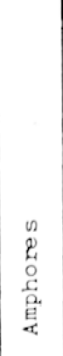 & $\begin{array}{l}\text { In } \\
\vdots \\
0 \\
0 \\
\text { H. }\end{array}$ \\
\hline c. 8 & & & & & & 1 & 2 & & 10 & & & & 15 & & & 3 & 9 & 2 & 39 & 885 & 64 & 1030 \\
\hline c. 9 & & & & & & & & & & 2 & & & 2 & & & & 1 & & 2 & 12 & 5 & 24 \\
\hline TOTAL & & & & & & 1 & 2 & & 10 & 2 & & & 17 & & & 3 & 10 & 2 & 41 & 897 & 69 & 1054 \\
\hline
\end{tabular}

Ta bifir: III. Nomenclature de la círamique provenant des remblais de l'espace I. 

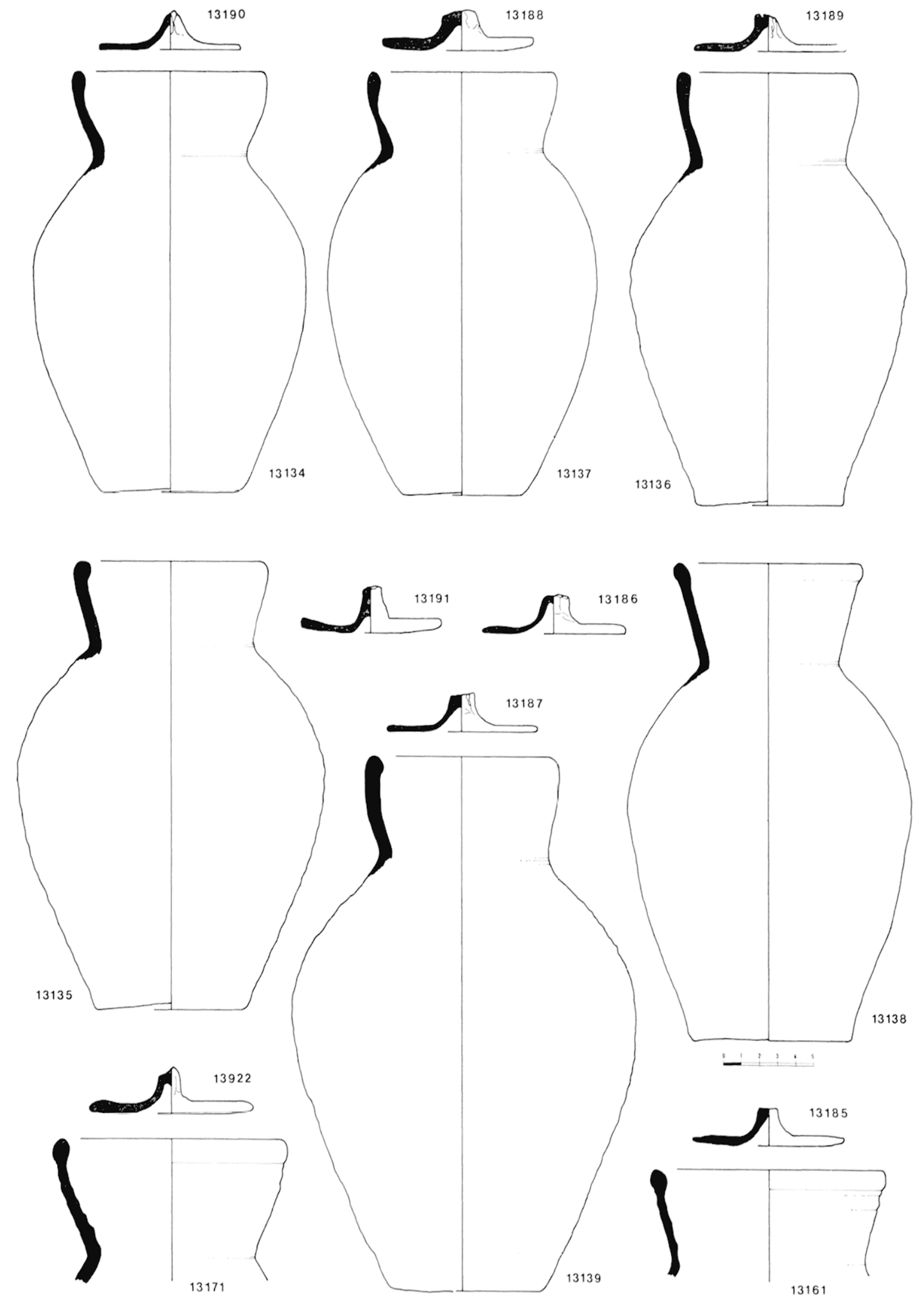

fig. 16 A. - pots du remblai de L'espace l (CF. fig. 15) 


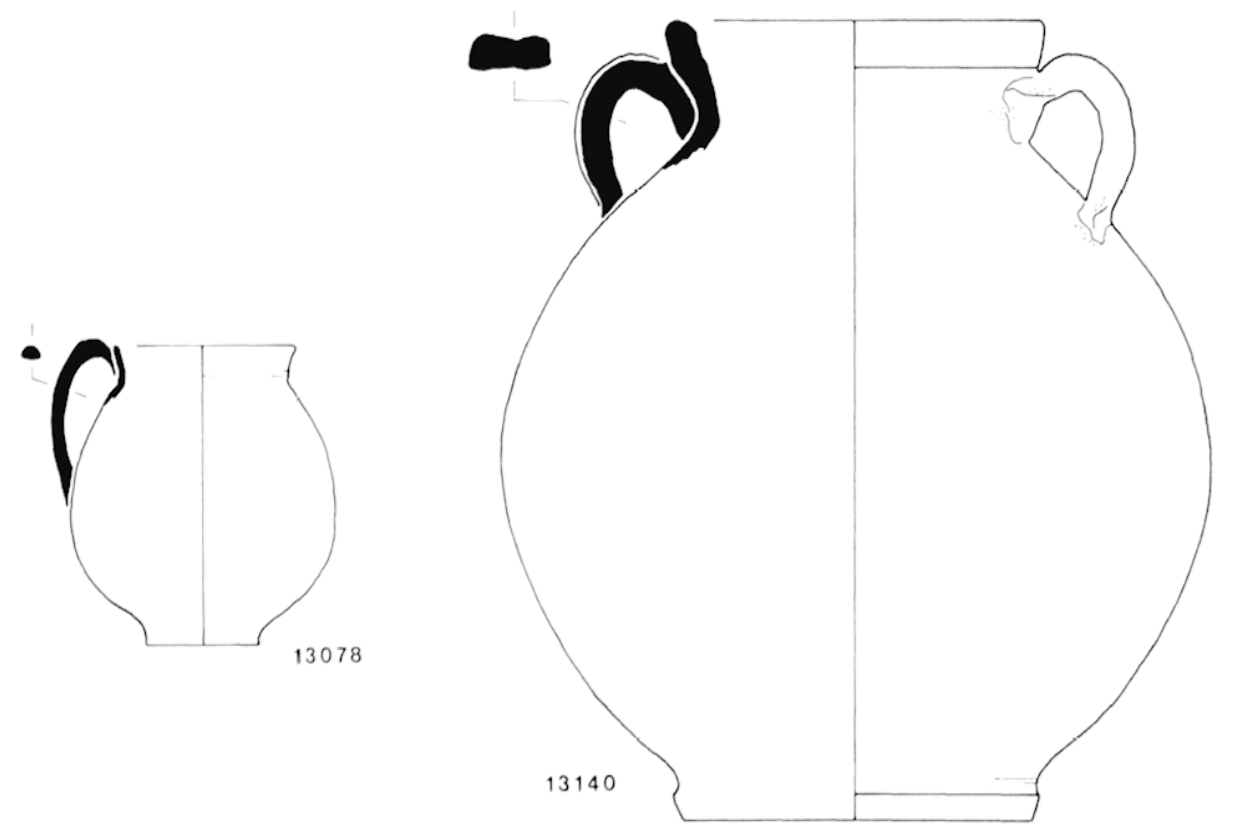

Fig. 16 b. - pot fé CÉramique commune a páte Claire et gobelet fi paroi fine PROVI:YANT DU REMBLAI DE L'ESPACE L

Par chance, d'autres secteurs du site ont livré des tessons identiques à ceux de cette zone en P5, C7 et surtout C2 (536 fragments), dans des couches 8 a ou $8 \mathrm{c}$ bien datées par le reste du matériel céramique vers le milieu du second siécle (cf. tableaux V et VI); c'est donc autour de cette période qu'il conviendrait, semble-t-il, de placer la production de l'officine.

La datation pourrait valoir par récurrence pour le remblai de la pièce $L$ qu'il faut peut-être interpreter comme le support particulièrement soigné d'un sol maintenant disparu et dont la cote nous échappe : vers 203,75 m., par analogie avec la situation des salles avoisinantes, $\mathrm{G}$ et surtout $\mathrm{M}$ plus au nord?

Dans la pièce $M$, la séquence stratigraphique est assez comparable à celle de la salle précédente. $\Lambda$ la cote $203,35 \mathrm{~m}$. ont été à nouveau repérées des tuiles - entières cette fois et disposées sur deux files parallèles - qui reposent sur un mince remblai de couleur orangée. La régularité de leur agencement et le soin apporté à leur pose font plutôt penser en ce cas à un véritable sol qu'à une surface de travail ; mais le niveau sur lequel elles ont été posées a été reconnu sur une trop faible surface pour qu'il soit possible d'être affirmatif.

$\Lambda u$-dessus des tuiles en tout cas a été déposé un remblai que le matériel céramique recueilli ne permet pas de dater de façon sûre : il est épais d'une quarantaine de centimètres et supporte un sol fait d'une couche de béton de chaux qui n'est conservé qu'au centre de la salle; en continuité avec cette surface, on trouve au nord de la terre battue et sans doute en allait-il de même dans la partie méridionale non fouillée car un niveau était clairement repérable à même cote - 203,75 $\mathrm{m}$. au long du mur 13, sur la paroi nord du premier secteur ouvert à la fouille.

C'est en tout cas en rapport avec ce même niveau qu'a été aménagé un égout domestique large de $0^{\mathrm{m}} 30$ qui nait au nord du mur 13 et traverse, avec un parcours d'ailleurs contourné, tout le secteur oriental de l'insula avant de passer sous le mur de façade disparu et de continuer sous le decumanus nord (34). Cet ouvrage en pente marquée du sud vers le nord $(2,25 \mathrm{~cm}$. $/ \mathrm{m}$. dans le bâtiment, $7 \mathrm{~cm}$. Im. sous le mur 45$)$ est de facture soignée : le fond est fait de tuiles posées sur une mince couche de mortier hydraulique épaisse de 5 centimètres; les parois, constituées de mœllons et de tuiles lices par du mortier sont épaisses de 20 centimètres et hautes de 25 et elles reposent partiellement sur les tuiles du fond; de la couverture enfin ne subsistent que deux fragments de tuiles (fig. 17).

Le sol de la pièce $N$ a entièrement disparu à cause de remaniements médiévaux d'ailleurs plus ou moins prononcés selon les endroits car la cote d'arasement des nombreux murs qui figurent dans cet espace (cf. fig. 19) est très inégale. Ne fût-ce qu'à cause de la présence de l'égout domestique qui la traverse en oblique, la pièce ressemble beaucoup à la salle $\mathrm{M}$; aussi restituerait-on volontiers ici un sol de même aspect (et de même cote?) que plus au sud.

\section{Les murs et les cloisons.}

I.FS MURS EN appareil tiatsonNé a l'argile.

Leur nomenclature est simple : ce sont ceux que nous avons représentés sur le schéma du premier état de l'insula I (fig. 4). Leurs fondations ont été déposées dans une tranchée large de $0 \mathrm{~m} 65$ environ dont le fond est à la cote $203 \mathrm{~m}$.; à la cote moyenne $203,35 \mathrm{~m}$., un ressaut d'une dizaine de centimètres de large placé le plus souvent sur la face méridionale des murs longitudinaux et orientale des murs transversaux marque le début de l'élévation. Celle-ci est faite de blocs mieux 


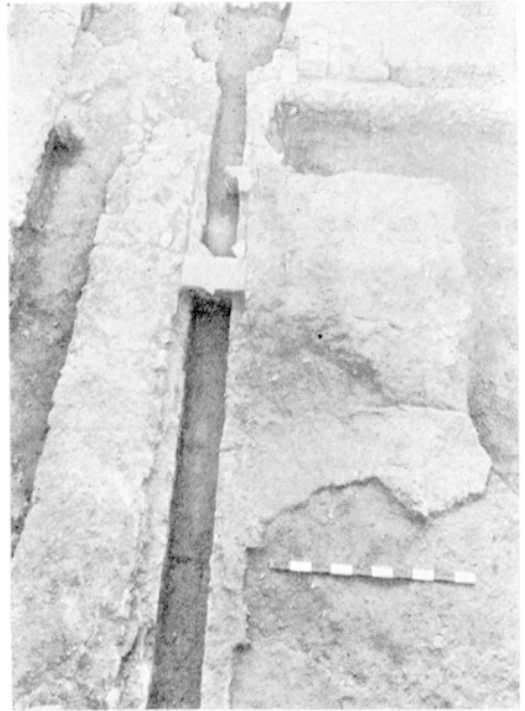

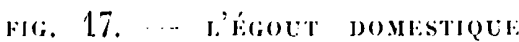

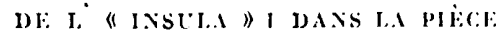
is, VI: De SCI

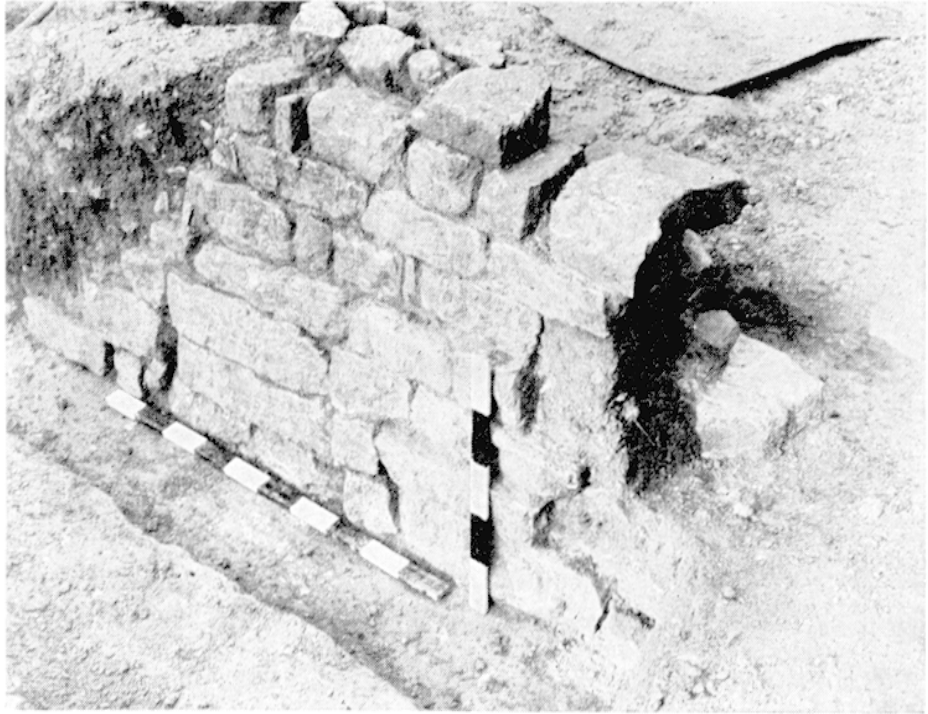

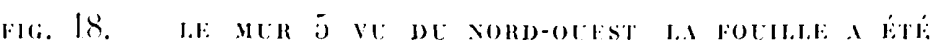

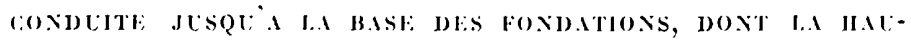

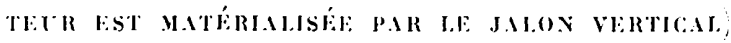

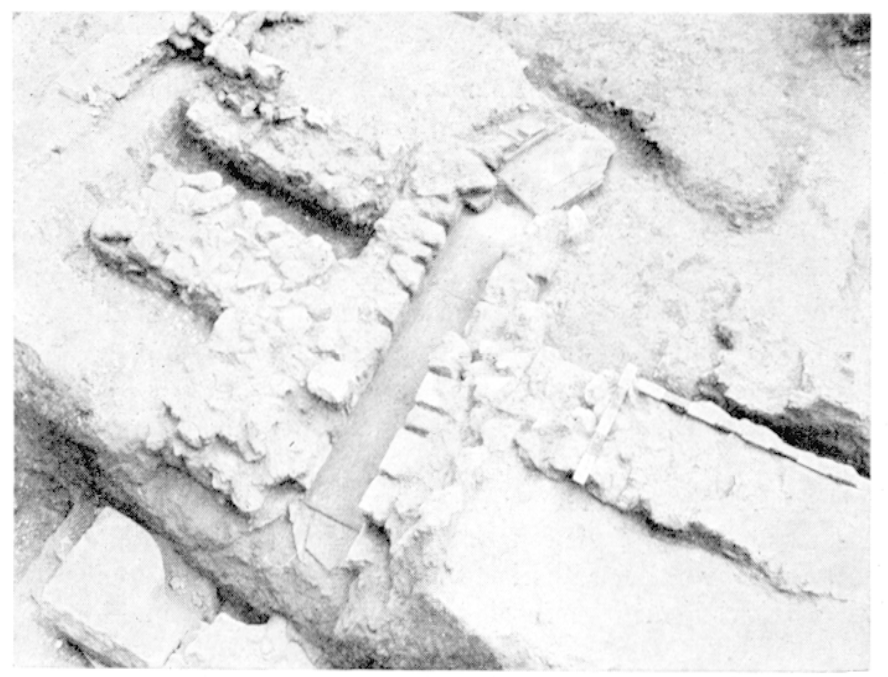

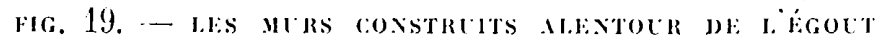

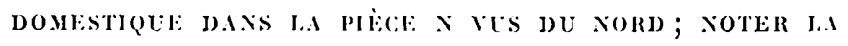

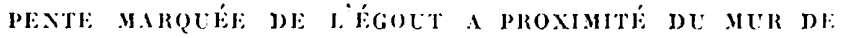
FAÇ.IDE DE L" "INSLIA " I DONT NE SUBSISTE PLCS, AT

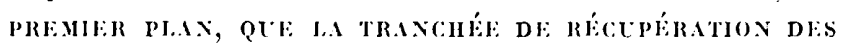
MATT́RAAUX

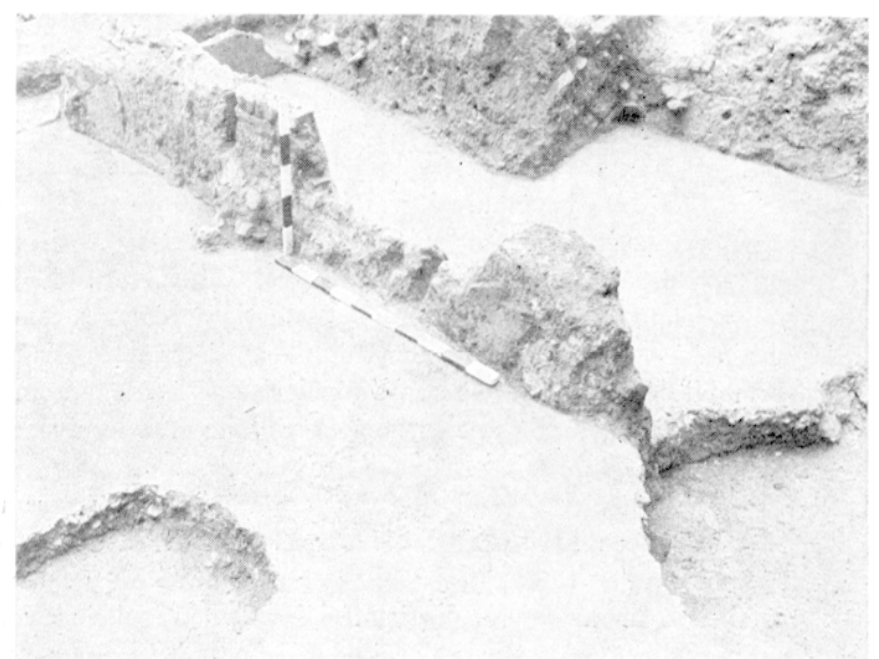

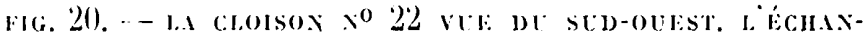

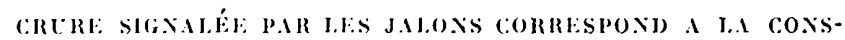

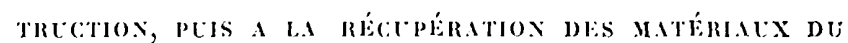
जer Mintivi, 76

équarris que ceux des fondations - mais non de véritables mollons; ils sont eux aussi liaisonnés à l'argile et disposés en assises relativement rigulières (fig. 18).

I,es altres ners.

Ce sont les murs des extensions méridionales et orientales do l'insula. Ils sont collés aux précédents et rarement liés entre eux - au moins au niveau des fondations. La tranchée de fondation est souvent plus étroite $\left(0^{\mathrm{m}} \mathrm{6}, 0\right)$ et toujours plus profonde que celle des murs voisins (en fonction de la pente du terrain et de l'importance des murs, la cote varie entre $202,90 \mathrm{~m}$. et $202,45 \mathrm{~m}$.) ; elle contient des blocs grossiers liés par un mortier de chaux de coulcur blanche ou rosàtre. L'ilévation, large de $0^{\mathrm{m}} 50$ n'est que très exceptionnellement conservee, et rarement sur plus d'une assise de hauteur.

Dans cette série, les murs des espaces .I et $X$ méritent une mention particulière. P'our marquer la séparation entre 
ces deux pieces, on peut hésiter entre les deux murs 21 et 23 dont le node de construction est identicue mais l'histoire différente. Ie mur 21, d'ailleurs fondé moins profondénent, allait initialement jusqu'au contact du mur 28 comme l'a montré un sondage au-dessous de l'égout domestique tandis que le mur 23 est lié au contraire à ce dernier onvage : faut-il penser que la construction du mur 23, pourtant fort étroit (son élévation ne semble pas dépasser en largeur 30 com.) appartient a une restauration contemporaine de l'établissement du systeme de drainage?

Plus au nord, un ensemble de murs médiocrement fondes (entre 203,32 et 203,92 m.; exceptionnellement $202,97 \mathrm{~m}$. pour le mur 37) a été construit autour de l'égout domestique (fig. 19). Les segments situes de part et d'autre de l'écoulement ne sont pas alignés, ce qui interdit de les associer pour en faire des murs de séparation au sein de la piece $X$; tous sont appuyes contre les murs longitudinaux, 28 et 3h mais tandis que les murs 333 et 35 sont aussi collís cont re les parois de l'égoul, 37 et 39 sont lies a elles et le mur 39 fait mene retour vers le nord. Faut-il associer tous ces idiments assez heterogènes? lit comment les interpreter? A cause de l'egout, faut-il penser à un dispositif de latrines?'

l.es cirorsons.

Au nord comme au sud de l'insula, des cloisons ont ete appuyées contre les murs maitres pour séparer les salles. La 1) hupart ont dete tres largement detruites $\left(2{ }^{\prime}, 26\right)$ ou rasees au niveau de leurs légeres fondations $(20)$; seule 22 est conservie en élivation. Latrge d'une dizaine de centimètres, elle est faite en colombage : voir sur la figure 20 l'empreinte laissée par l'appareil en bois disparu; les hourdis étaient constitues d'argile crue liant des matériaux de récuperation (moellons et fragments de tegulae). Sille est revitue d'un enduit de couleur rouge que l'on retrouve aussi, sporadiquement, sur les autres murs et cloisons de l'édifice.

\section{Shitis mincelatiox,}

Quatre seuils sont conservés. Les deux plus au nord ont seulement éte repérés lors de l'aménagement de la partie de la Cour de l'Archereché aujourd'hui occupee par les gradins du thèatre. Placés perpendiculairement l'un à l'autre à l'angle nord-est de l'espace $\%$, ils sont très voisins par leurs dimensions $\left(1^{\mathrm{m}} 6.5 \times 0 \mathrm{~m} 50\right.$ environ $)$ et commandent l'accies aux salles 12 et B. Ies autres seuils, au sud, ont pu être mieux éludiés. Ils ouvent tous deux sur le decumanus à partir de la pièce J'i ; le plus a l'ouest laisse un passage libre de $2^{\mathrm{m}} \mathbf{1}^{\prime} \mathrm{t}$ (pour une ouverture totale de $3^{\mathrm{m}} 2 \mathbf{t}_{\mathrm{t}}$, piedroits de la porte compris : cf. fig. 14t) et se compose de deux blocs; le seuil oriental, reservé à l'usage pieton, est un monolithe de lm 10 de long. Les blocs en pierre froide ont une hatuteur maximale de $0^{\mathrm{m}} 30$ et reposent sur les fondations du mur 1 par l'intermidiaire d'une épaisse couche de mortier; ils gardent l'empreinte des crapaudines el même, pour celui de l'ouest, du dispositif de fermeture.

Il n'est guere possible de reperer ailleurs des arrachements ou des vestiges de seuils - sauf peut-être en deux endroits: sur le decumanus encore, à hauteur de la sallr. 22 où les deux segments conserves en clévalion du mur 1 semblent bien être des têtes de mur et entre les pièces J't et $K$, où l'appareil du mur 4 parait marquer lui aussi un arrêt franc (pour un piédroit de porte?).

\section{b) Datation.}

Pour dater létat acheve de l'insule I, il faut se lourner d'abord vers la rimanique des remblais au-dessous des sols en béton qui ont pu être foullés. Ie tableau suivant en donne la nomenclature.

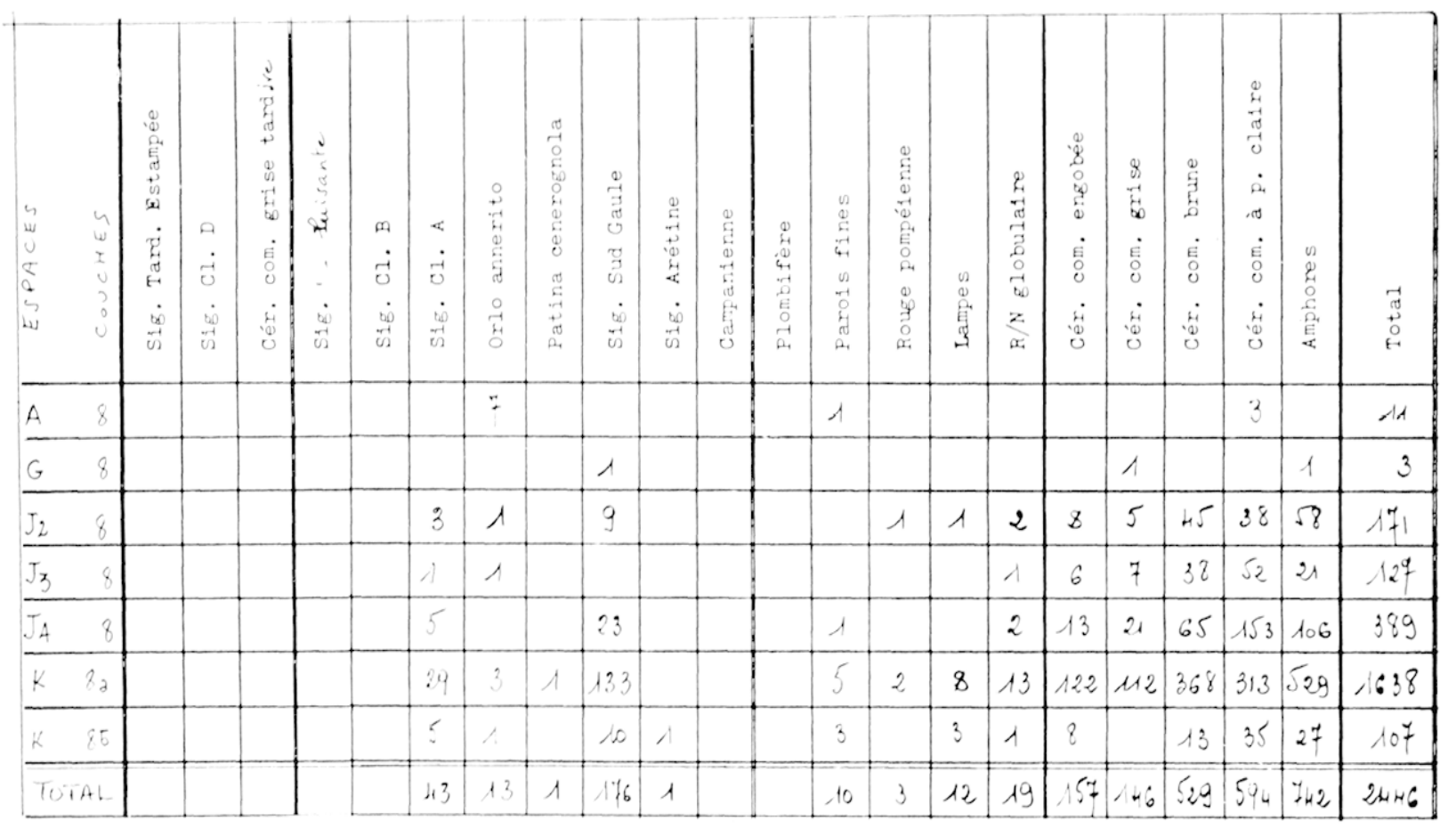

Tabriau IV. Nomenclature de la céramique provenant des couches scellées par les sols en béton de l'insula I. 

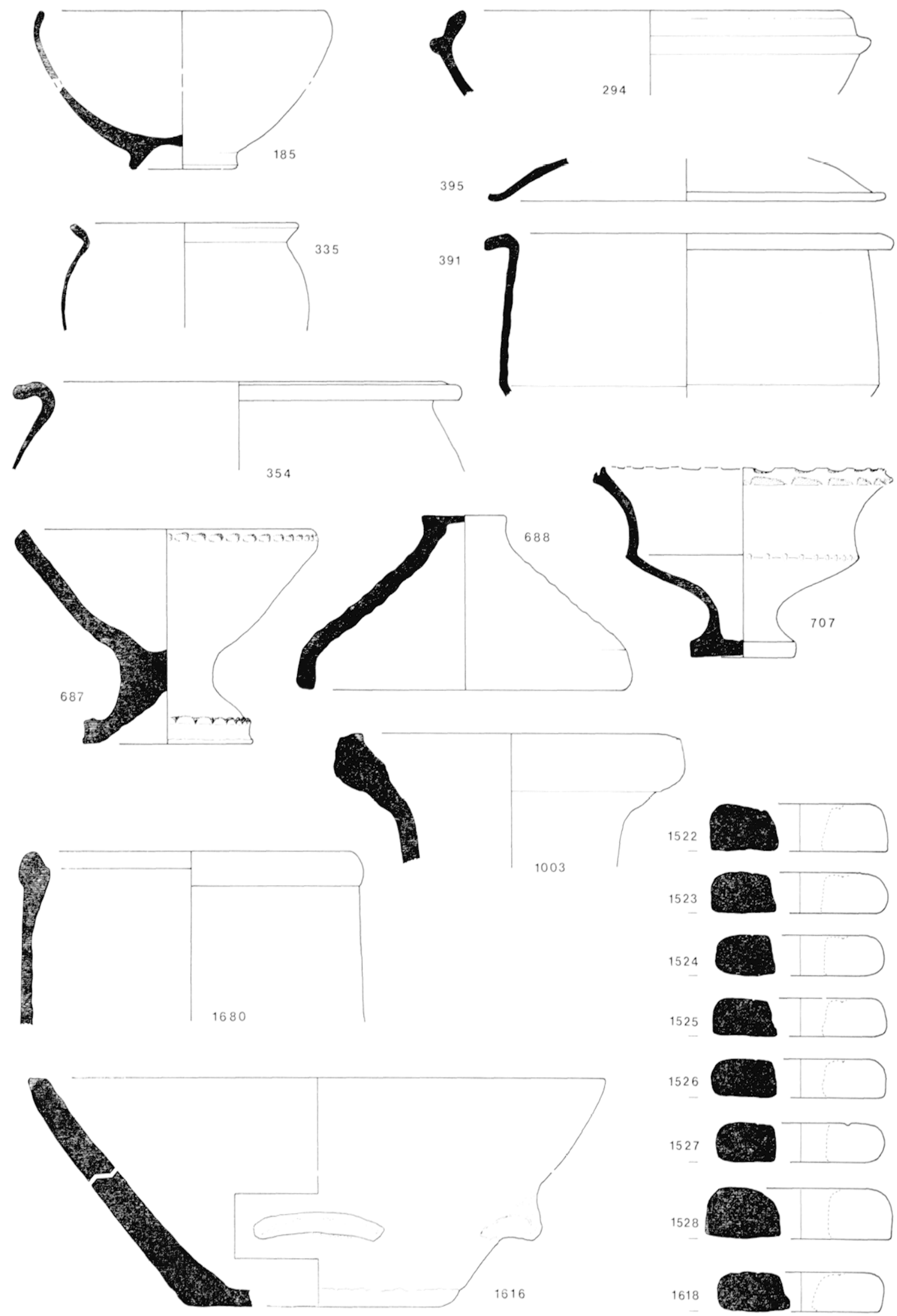

003

1523

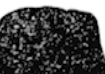

1524

1524

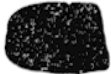

1525

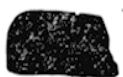

1526
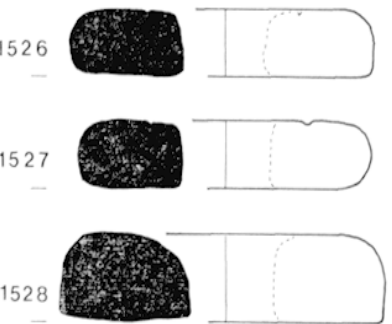

1618

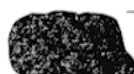

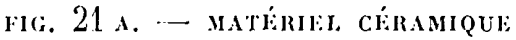

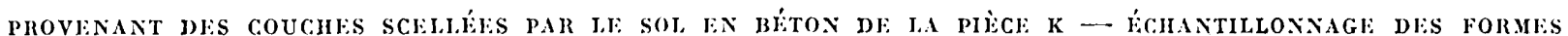



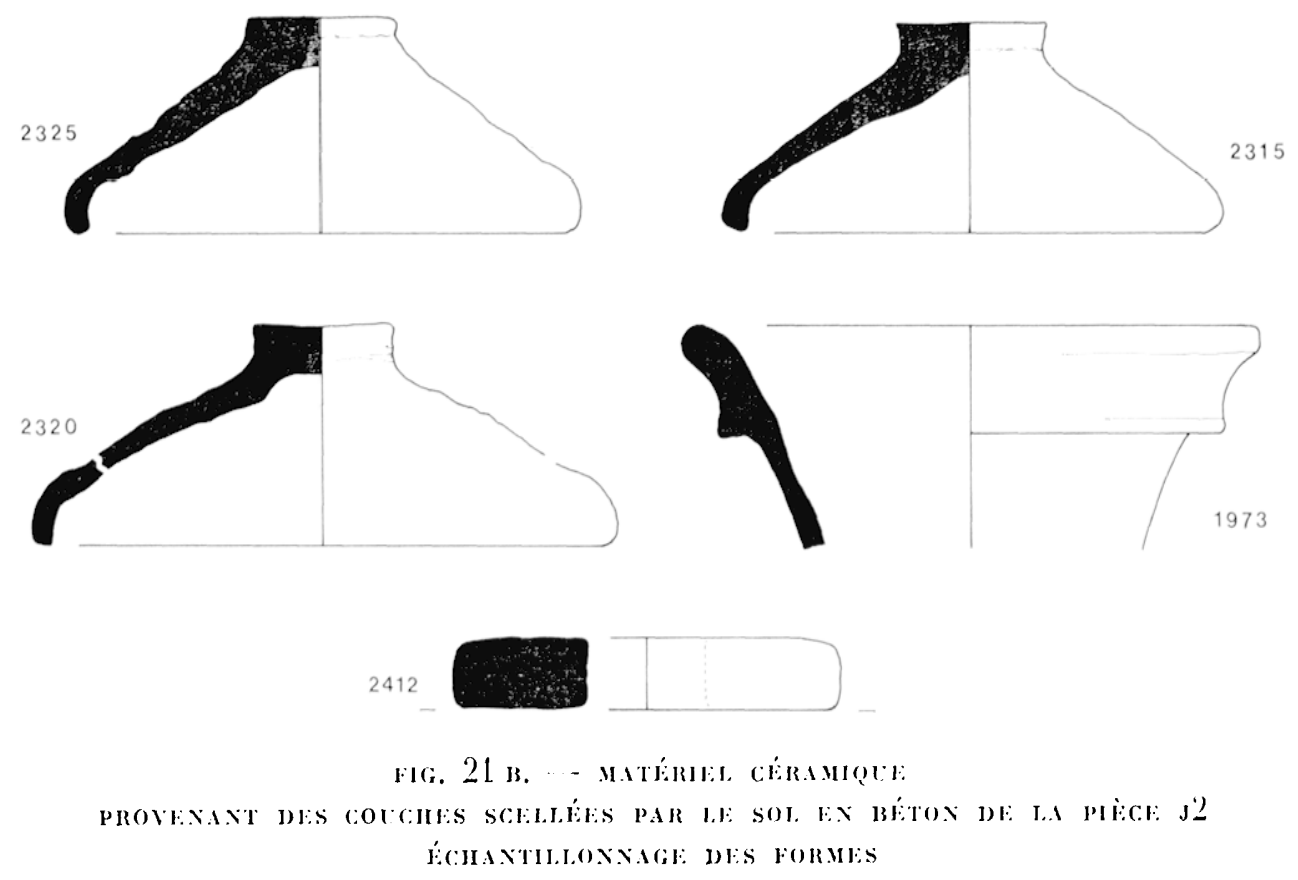

L'essentiel du matériel - dans une proportion de $71,3 \%$ \% - provient de la pièce $K$, la seule qui presente des traces d'un remaniement important des sols 33 j et enci pourrait expliquer relat dans cet espace dailleurs, le remblai 8 a repese sur la couche endrense 8 b qui reeoure largile vierge et que nous avons dijat sommairement

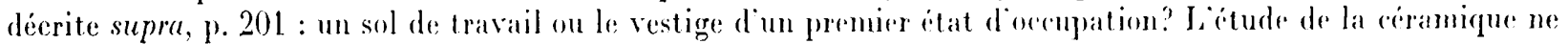
permet pas de trancher entre ces deux interpretations car le faciès très semblable que présentent les deux strates peut s'expliquer par l'intrusion de tessons du remblai dans la mince couche sous-jacente.

Les profils céramiques de la figure 21 A donnent un bon échantillonnage du matériel le plus original. Dans la couche 8 b, on notera surtout lat présence de quatre éléments de bassins en crimamique modelée avee ansers appliquées (no 1610) qui étaient écrasés sur le sol et dans la couche d'abandon du fover de l'angle sud-est, une léve d'amphore (1680) qui peut se rapprocher de la forme Dressel $2 / 1$. Dans la couche 8 a ligurent d'autres fragments d'amphores des formes Crauloise 5 , Dressel $2 / 4,7 / 11$ et 20, des anses de type rhodien lardif at des lères de forme.

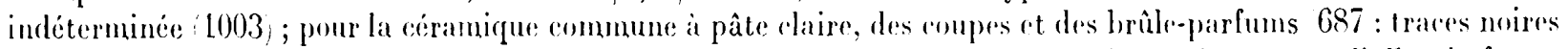
a l'intérieur; 707) ainsi que des eouvereles 688; pour les pâte's brunes, de nombrenx fragments d'ollee de forme:

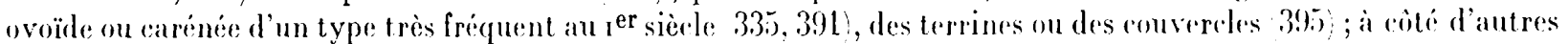
ollue et terrines, la éramicue grise livre une originale coupe a collerette 291 tandis que dans la céramique commune engobee, l'essentiel du matériel est constitué de coupes de tradition augustécone 18:). On remarquera enfin une série de huit disques en terre cuite nos l:222 et suivants - .. sans doute des cales de potiers.

La céramicque sigillese du Sud de la Gaule, non représenties sur la figure 21.1 , fournit un fort contingent de

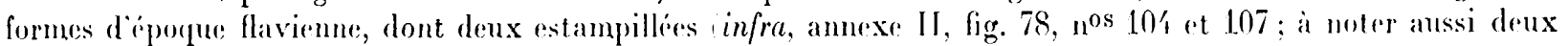
marques sur lampes, nos 165 ) $(166)$; mais les éléments de datation de la rouche 8 a sont fournis par la sigillée claire 1 : denx fragments de forme fermée, des tessons de forme Lamb. fa 10 . $\mathrm{det} 10 \mathrm{~B}$ a strisce et un fragment de couvercle Lamb. 20 -... autant d'écéments qui permeturaient de placer au plus tiot la constitution du remblai à

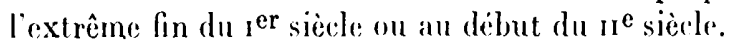

L'étude des couches 8 des autres espaces confirmerait we jugement, car le matériel fig. $21 \mathrm{l}$ y y est ues comparable 36 mais la présence sous le béton de la pièce $\mathrm{J} 3$ d'un as posthume de laustine 1/1-161 infra, appendice numismatique, $n^{\circ} 18$ conduit à rajeunir sensiblement la datation. Loin d'être exeeptionnel, le phénomène est presque une constante sur ce site où nous le retrouverons à propos d'autres couchers, antiques et médiévales ; il conduit à penser que c'est après le milién du ${ }_{1}{ }^{\mathbf{e}}$ siècle de notre ère que less sols de l'insula ont été refaits : non seulement les surfaces a béton que nous venons de dater mais aussi peut-être less sols plus frustes de tout le serteur oriental pour lesquels la reramique commune contenue dans les remblais sous-jarents fournit un terminus ante

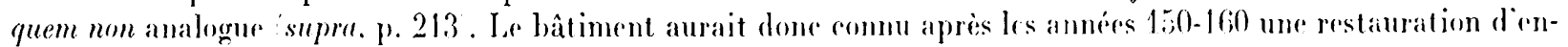
vergure. 


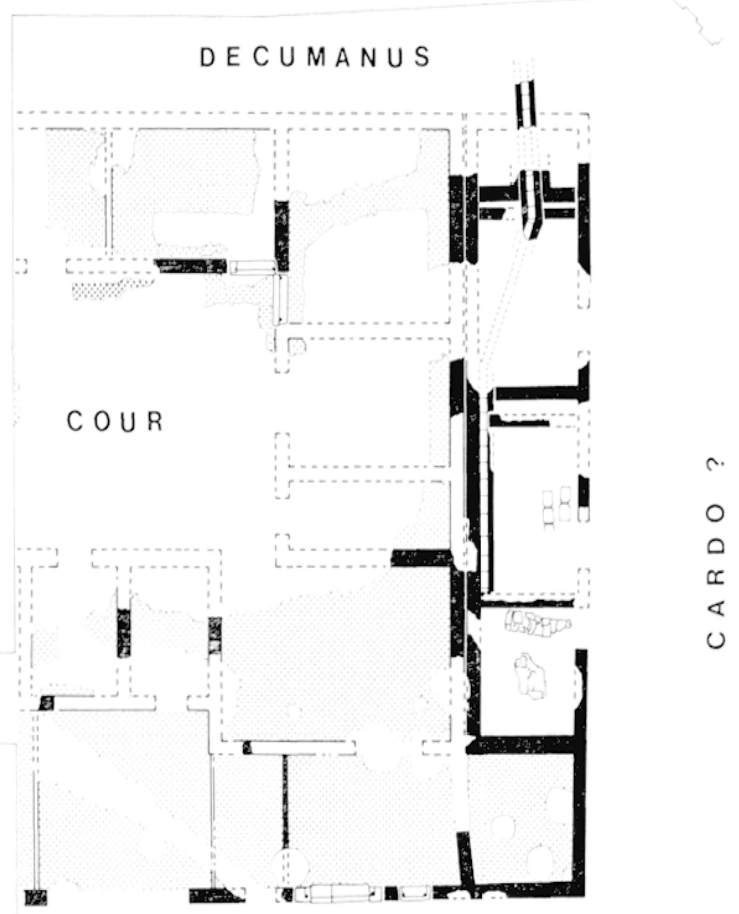

DECUMANUS

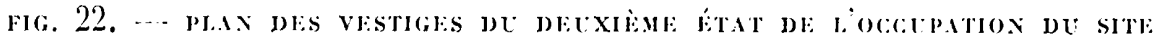

c) Restitution el interprétation.

Restrtertox.

Malgré la récupération des matériaux de la plupart des murs, la bonne conservation des sols en beton permet de lire très largement le plan de l'édifice. Aussi le schéma de la figure 22 ne diffère-t-il guère du relevé général de la figure 2 que par la présence de tout un réseau de circulation, pour partic conserve cet pour partic hypothétique. Nous avons indique quels sont les élements sûrs fou hautement probablesł en ce domaine isupra, p. 21.5, seuils et circulationj; il reste a justifier les partis pris pour nos restitutions.

Deux faits nous ont guidés dans notre reconstitution : la présence des deux seuils à l'angle nord-est de l'espace $\%$ et le doublage des murs 2 et 28 a l'est de l'insula. Ise premier invite d'autant plus à considerer l'espace \% comme un espace ouvert que l'existence d'une cour est nécessaire au centre du hâtiment pour assurer a la fois léclairage et la ventilation : aussi la plupart, sinon l'ensemble des pièces alentour de cette cour devaint-elles communiquer avec elle; peut-être seulement le côté est était-il précédé d'un portique dont le mur que nous avons entrevu au printemps de 198 a au sud de la pièce 12 aurait gardé la trace? Ia forte césure marquée par les deux murs 2 et 28 nous a poussés d'autre part à considerer l'ensemble du secteur oriental comme un tout et cest pour- 
quoi nous avons supposé qu'il n'était aceessible qu'a partir de l'est. Lees autres ouvertures ont été dessinées pour permettre la circulation dans le reste d'un édifice aucquel nous n'avons donné, faute d'indices, aucun dégagement vers le nord, supposant le mur $\$ 3$ aveugle.

Line restitution en élévation est plus problématique, s'agissant d'un monument ruiné presque partout jusqu'au niveau des sols; aussi l'axonométrie de la figure 23 n'a-t-elle qu'une valeur purement indicative. Sur ce schéma, nous avons pris le parti de ne dessiner qu'un rez-de-chanssée parce que nous n'avons trouré trace nulle part d'un éventuel étage et qu'aucun espace n'est assez étroit pour que l'on puisse songer à restituer à son emplacement une cage d'escalier. De l'romsemble des toitures qu il est aisé de couvrir par des charpentes du type te plus classique n'omerge done que Ir volume supporte par les murs de la pièce fo auquel nous avons réservè un traitement particulier a cause de ses vastes dimensions 37: : peut-être y avait-il at cent rendroit un grenier acessible a partir d'une échelle?

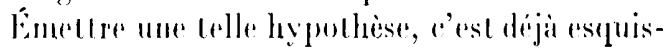
ser une interpretation d'ensemble de la fouille de l'insula.

\section{Ixterpiétatox.}

Pour comprendre la nature des bâtiments mis an jour dans la Cour de l'Arehevêché, il importe de les rapprocher des autres demeures aixoises que le's fouilles ont fait connaître depuis le delubt du $\mathrm{xrx}^{\mathrm{e}}$ sièle ; or, rien n'évocque ici les riches domus de l'lire du Chapitre maintenant parking l'astrure, du Jardin depuis conte de Cirassi on de l'Ecole des Beaux-Arts et du pavillon Vendome 38 qui, toutes, ont des sols décorés de mosaïques et s'ordomenent lo plus souvent autour de cours a péristyle avec lesquelles la cour retrouvée au sein de l'insula I n'entretient que de pâles st

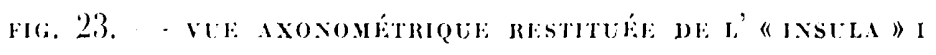
PRISE JU SCD-EST DESSIX : J. BIGOT: lointains rapports d'analogie.

A considérer ici les aménagements soignés mais sans luxe de toute la partie orientale du bâtiment, on songerait assez à une utilisation artisanale ou commerciale de tout ce secteur de limmeuble tandis que les grandes aires bétonnées de toute la partie méridionale conviendraicnt mieux à des cutrepôts qu à une véritable demeure: plaiderait assez en ce sens le pendage régulier et accusé des sols en direction de la cour ou de la rue, qui facilitait leur nettoyage.

Sauf pour la salle G, la même remarque ne s'applique pas cependant aux pièces situces autour de la cour qui ont d'ailleurs pour la plupart des dimensions moindres; aussi est-il possible qu'elles aient servi pour leur part à l'habitation. Comme ces pièces sont toutes situées au sein de l'aire couverte par le premier état de l'insula lef. fig. 4i, il est permis de se demander si les extensions - certaines - du bâtiment n'ont pas consisté surtout à adjoindre entrepôts, ateliers ou boutiques à une première maison d'habitation du type le plus classique dans l'urbanisme romain, mais la fouille nous renscigne trop peu sur les débuts de l'insula et sur ses transformations pour qu'il soit possible de répondre de façon tranchée à cette question.

Dailleurs, l'enquête archéologique du chantier de l'Irchevêché ne nous autorise pas plus à conclure sur l'évolution de ce secteur qu'à brosser de façon définitive le plan d'urbanisme d'Aquae Sextiae à la fin du second siècle de notre ère : sans doute à cette date les bâtiments du centre urbain ont-ils pauvre apparence en regard des villas urbaines qui semblent avoir couvert tous les quartiers au nord de la ville, mais l’impression suggérée par les fouilles, malgré tout restreintes, faites ici et lia doit-elle tout au hasard ou répond-elle à la réalité? Lu moins est-il sûr qu'à la lin du siècle des. Antonins, le plan d'urbanisme réglé des abords du forum abritait certainement, dans 


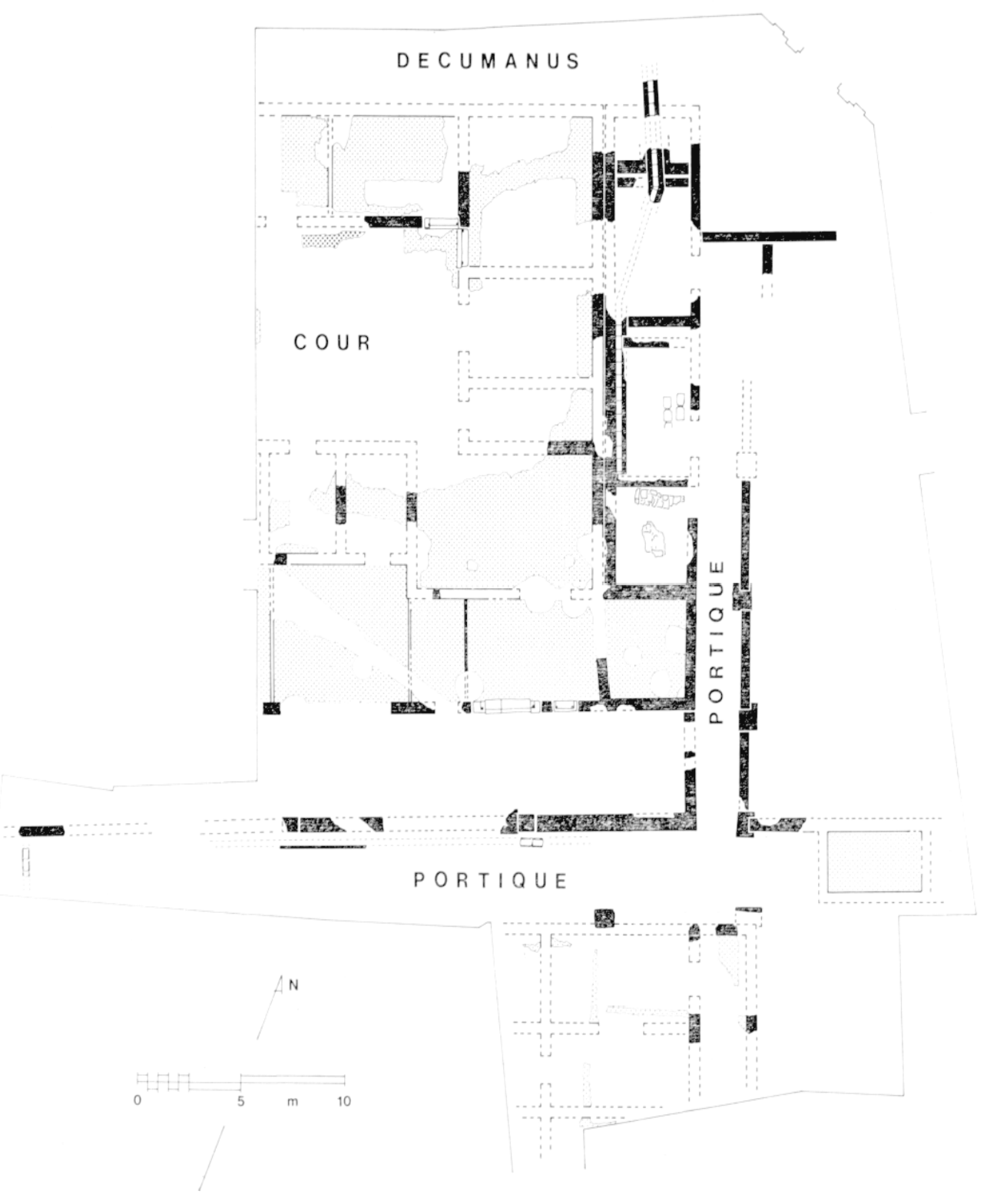

FIti, 24. - PIAX DISS VESTIGIS

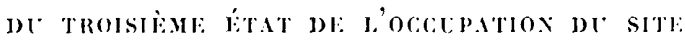

l'insula I, non pas l'otium des plus riches mais la vie de leurs clients ou de leurs esclaves. Le trait peut servir a comprendre l'évolution de la voirie avoisinante.

IV. - LES TRAXSFORMATIONS DI: LA VOIRIE

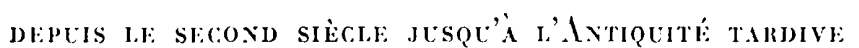

Rien de plus changeant que le paysage d'une rue; nous le vérifions tous les jours. Il c'n allait de même dans l'Antiquité mais pour le percevoir, il nous faut renoncer à pratiquer des coupes chronologiques comme celles que nous venons de faire à la fin du ${ }^{e r}$ siècle ou vers le milieu du ${ }^{\mathrm{e}}$ siècle, à des moments privilégiés où tout un quartier ou un bâtiment entier venaient d'être restaurés. Nous déerirons au contraire maintenant l'ensemble des modifications qui ont affecté les chaussées : ce sera l'occasion de vérifier que les transformations de la voirie ont étí continues, qu'elles ont peut-être commencé avant que ne fùt achevée la restauration de l'insula I que nous venons de signaler et qu'elles se sont poursuivies bien après, marquées à la fois par des exhaussements du sol qui témoignent 
d'un long entretien et par l'apparition de constructions adventices qui ont peu ì peu accaparé l'essentiel du domaine public (fig. 2/).

\section{a) Description.}

Exinussement des chaussírs.

$\Lambda$ u-dessus des niveaux de cailloutis ou de béton grossier de la fin du $\mathbf{1}^{\mathrm{er}}$ siècle (supra, p. 205), nous n'avons nulle part retrouvé sur les chaussées des revêtements d'un faciès comparable mais seulement des sols en terre battue, d'ailleurs assez soignés et dont le profil permet d'autant mieux une bonne évacuation des eaux de ruissellement que la pente du boulevard (?) à l'est du site suit celle du sol naturel : la circulation se fait désormais au nord, en C2, a la cote 203,80 m. et au sul, en ('t', autour de $203,55 \mathrm{~m} \quad(39)$.

Ces niveaux de terre battue seellent de nombremses et rastes fosses qu' il faut des maintenant signaler, tout en renvoyant pour l'essentiel de leur étude au developpenent consacré à la datation, infra, p. 223 sif. Ce somt de viritables dépotoirs qui contiennent, outre des depots céramiques abondants et sourent grossiers iplus du quart des tessons du site, avec une predominanee de fragments d'antphores), tout un matiriel heteroclite : clous en fer fenviron 200), placques ou ielats de marbre (120), tesselles de mosaïgue fplus dr 200), fragments de dolia (une centaine) et rebuts d'instrumentum domestirum, fibule, fil a plomb, plateau de balance, miroir, ipingles, etc. $(40)$ : sans doute des recharges pour le sol des chaussees dont nous avons signali la relative qualité.

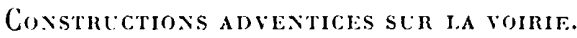

- Les dés en maçonnerie.

La fouille a révélé l'existence sur les chaussées ou leurs abords de six (et peut-être sept) massifs de maçonnerie (cf. fig. 2't) : de section carrie ou rectangulaire, ils mesurent

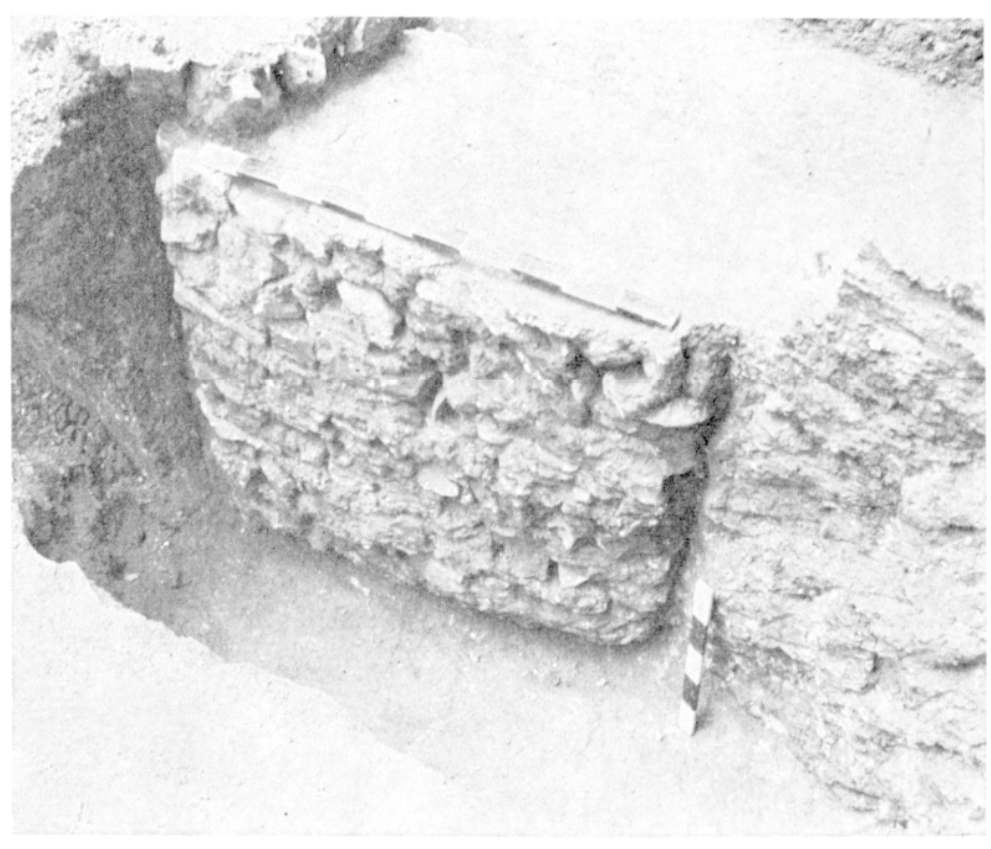

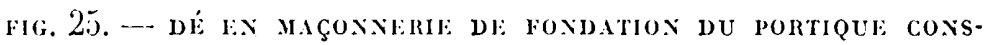
TRLT DEVANT IA FAÇADE FST DE L' "INSLLA " I VU DU XORD-

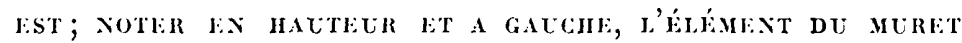
36 coxstrutT AU-DEssus dU DÉ DE FONDATION environ 1 mètre carré de superficie et sont profondément fondés entre 202,65 et $202,35 \mathrm{~m}$. (fig. 25 ) ; leur arase supérieure, de mêrne, est généralement à une cote assez hasse - autour de $203,35 \mathrm{~m}$. : il s'agit manifestement de dés de fondation.

Les massifs dessinent trois alignements. Celui de l'est est de direction nord-sud et comprend quatro éléments : les deux premiers sont placés devant la façade orientale de l'insula I, 2 mètres environ à l'est du mur 30 et sensiblement au droit des murs 3 et 7 ; le troisième est presque dans l'axe du decumanus sud et le dernier, à l'emplacement de l'angle nord-est restitué de l'insula II ; il n'a d'ailleurs été que particllement reconnu sous le mur sud des écuries du xvin ${ }^{e}$ siècle, qui passe à cet endroit et recouvre également en partie un second massif situé environ 6 mètres plus à l'ouest : la direction générale du mur moderne dessine donc un second alignement sensiblement perpendiculaire au précédent. Un dernier alignement, également orthogonal au premier, est constitué par le (ou les) dés placés entre les différents segments du mur 51 (41) qui occupe en gros l'axe du decumanus, au droit du troisième élément de la première série.

. Murs et sols en liaison avec les dés.

Ces alignements sont matérialisés aussi par des murs qui semblent associés à chaque fois aux massifs maçonnés. Le doute est seulement permis pour la série méridionale en lisière de l'insula II mais la présence des lambeaux des sols en béton de cet édifice qui ont été repérés presque jusqu'au contact des massifs (cf. fig. 2'́t) incite à penser que le mur moderne des écuries (sous lequel nous n'avons pu fouiller) était fondé, au moins pour partie, sur le mur de façade de l'insula et si l'interprétation est juste, il faut admettre que le mur antique lui-même, 57, recouvrait, au moins partiellement, les massifs maçonnés (ce qui suppose plusicurs étapes - dont la chronologie nous échappe totalement - dans la construction ou les réfections de cette insula).

Dans le premier alignement de direction nord-sud en revanche, le doute n'est pas permis car des segments de murs de largeur et de direction d'ailleurs légèrement différentes, 36 , empiètent partiellement sur les massifs maçonnés : il s'agit de constructions légères, peu profondément fondées (vers 203,10/203 m.), destinées à relier entre elles les élévations portées par les dés et à séparer nettement deux zones au sein de l'espace qui s'étend à l'est de l'insula I. Tandis qu'à l'est du mur se 


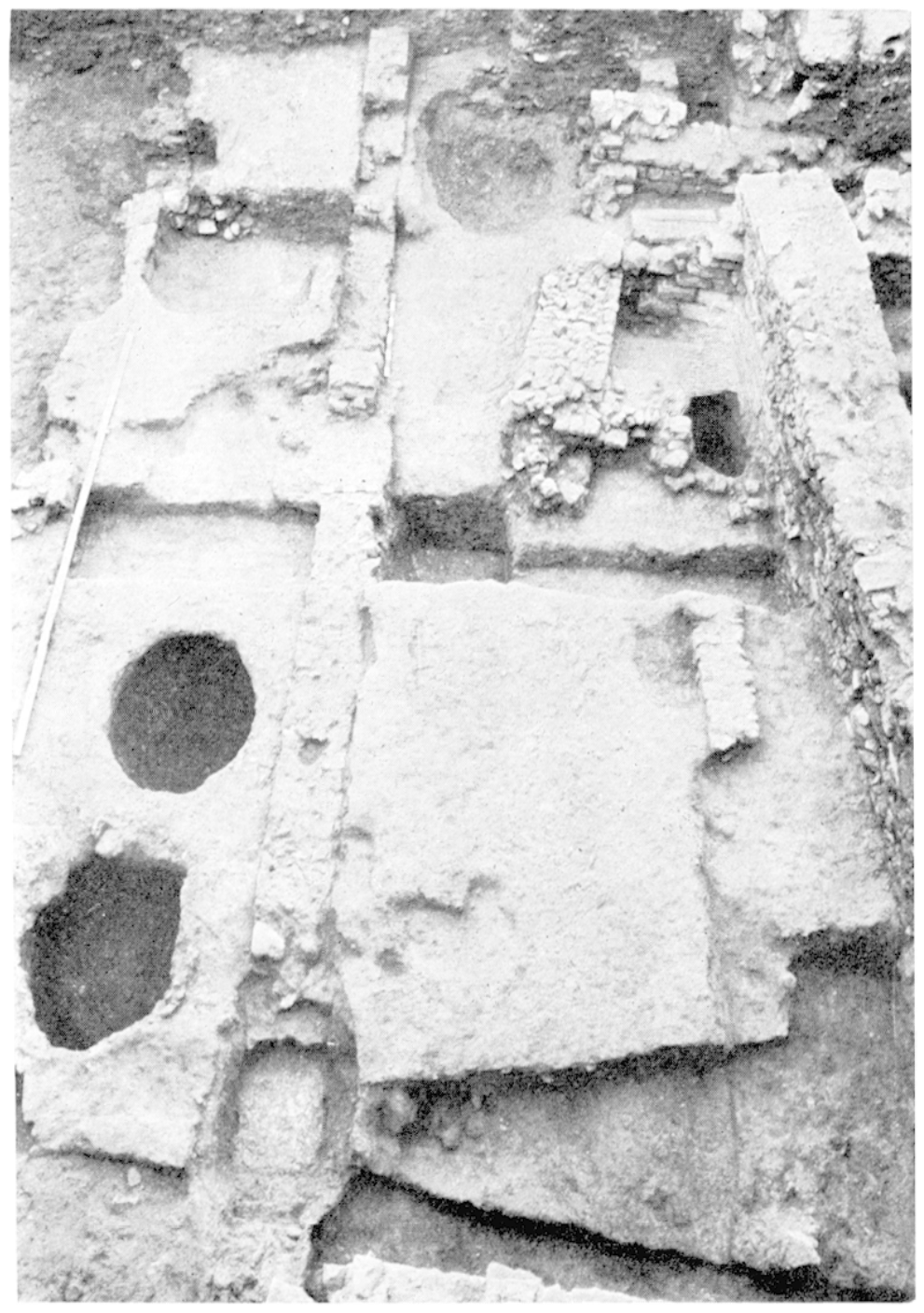

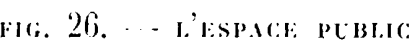

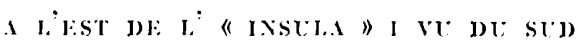

I gauche (jalon), mur de façade de l'insula et sols du portique; au centre dis de fondalion et mur bahut 36 du portique; a droitr, chaussee en terre battue du boulevard (a) partiellement masquie par des constructions plus tardives (noter en hatut la direction oblique du mur 68, qui est celle du passage médieval à cet endroit; en b. et a droite, le mur 52 et à l'extrême droite, les restes du mur de direction nord/sud représenté sur le dessin de Belleforest : cf. fig. 70).

retrouvent en effet les niveaux de circulation en terre battue que nous avons déjà décrits, à l'ouest au contraire ont été repérées deux surfaces chaulées directement superposées au cailloutis de la chaussée du ${ }^{\text {er }}$ siècle (fig. 26) : ces niveaux, d'apparence soignce, correspondent à des aménagements successifs d'un sol situé d'abord à la cote $203,47 \mathrm{~m}$. puis à $203,57 \mathrm{~m}$. (donc en contrebas de la chaussée dont le revêtement a été excavé à leur emplacement) et ils sont présents aussi bien à l'est de l'insula que sur le decumanus, dans l'cspace P5.

Nous avons d'ailleurs retrouvé des sols comparables par leur aspect et par leur niveau dans tout le secteur méridional de la rue au sud du mur 51 , surtout dans l'espace P7 où les couches étaient bien préservées mais également en P6 où les transformations médiévales et modernes ont laissé subsister des traces d'un niveau au moins, à la cote $203,49 \mathrm{~m}$. I.e mur 51 paraît avoir ainsi marqué une césure aussi importante que l'alignement des murets 36 car la situation, dans ce cas encore, est différente de l'autre côté de l'ouvrage où semble subsister un niveau de circulation autour de la cote 203,11/203,50 m.

Le mur 51 difière sensiblement pourtant des murets précédemment décrits : il est profondément fondé (autour de $202,15 \mathrm{~m}$.) et ses différents segments sont parfaitement alignés avec les dés qu'ils réunissent - deux traits qui suffisent à expliquer que l'individualisation des dés soit parfois problématique ici (d'autant que nous avons dû nous contenter souvent d'une reconnaissance superficielle des appareils) ; il s'agit en outre d'une construction très soignée qui fait retour vers le nord pour s'appuyer contre l'angle sud-est de l'insula I : l'élévation, conservée en deux endroits, est large de $0^{\mathrm{m}} 30$ environ seulement et les deux parements sont revêtus d'un enduit rouge analogue à celui qui recouvre le mur de façade de l'immeuble (fig. 27).

Un égout longe le mur au sud; à cause de l'importance des récupérations médiévales, il n'en reste que quelques traces : deux tuiles posées à plat dans l'espace P7, une partie du parement sud à hauteur de l'espace D2 ; sans doute s'agissait-il d'une construction assez comparable à l'égout domestique de l'insula I, donc large dans cuvre d'une trentaine de centimètres (cf. fig. 17). Son écoulement semble avoir été dirigé vers l'ouest : faut-il le mettre en relation avec une autre section d'égout, de direction nord-sud cette fois, que nous avons aperģue à l'extrême ouest du chantier, en P6 lors d'une reconnais- 
sance très rapide? et où placer son origine : à l'angle du mur 51 ou plus à l'est, où se trouvent d'autres coustructions de même alignement qui ont empiété plus largement encore sur l'espace ouvert situé ì l'est de l'insula $\mathrm{J}$ ?

Autres constructions.

L'espace ouvert de l'est est en effet barre au sud par un muret, i9, situe dans l'exact prolongement du mur 51 , qui semble bien avoir maré lui aussi une césure importante daus le site. Ce jugrment relive plus pourtant de l'interprétation que de la simple description car le muret n'est conserve - et mediocrement - que sur une longueur de moins de 2 mitres a son extremite ouest où il empiète sur l'un des des de fondation du premier aligrnement que nous avons décrit; mais il suffit te le prolonger idealement en direction de l'est pour observer que sa limitr mépidionale correspond exactement a l'arrêt frane d'une aire couverte d'un sol en biton de tuileaux, qui a bli reconnue dans des conditions tries difliciles sous la piree surdest des eccuries du xvere siecle : il anrait doue pu barrer an nord

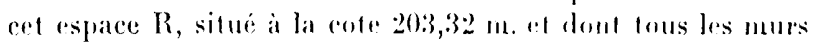
limitrophes ont d'ailleurs disparu.

L'are mise an jour lef. fig. 2) pourrail correspondere

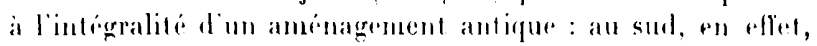

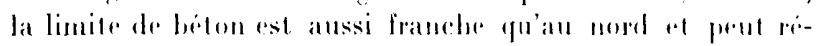

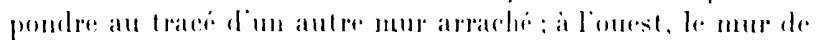

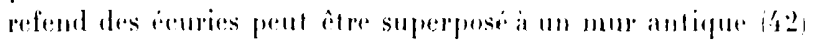
tamlis qu'al'mst on le sol se poursuit crertainment au-lessous de la paroi moderme, lat prísence diun bloc de grand appareil pris dans le beton pourrait marguer la limile primition d'une construction ulterieurement intondue an direction de l'est.

Il est inutile de s'altarder sur de's vestiges aussi mal conmus et d'ailleurs difficiles a dater ('33) et l'on observera surfout qu'au nord du mur 49, mulle aire bétomne analogure n'a ilir retrouvier : de la, la supposition que le mur marque bien une crisure importante sur le cardo. Ce n'est pourtant qu'une supposition car les perturbations midievales, nombreuses dans ce secteur, peurent expliquer une lacune; mais pour-

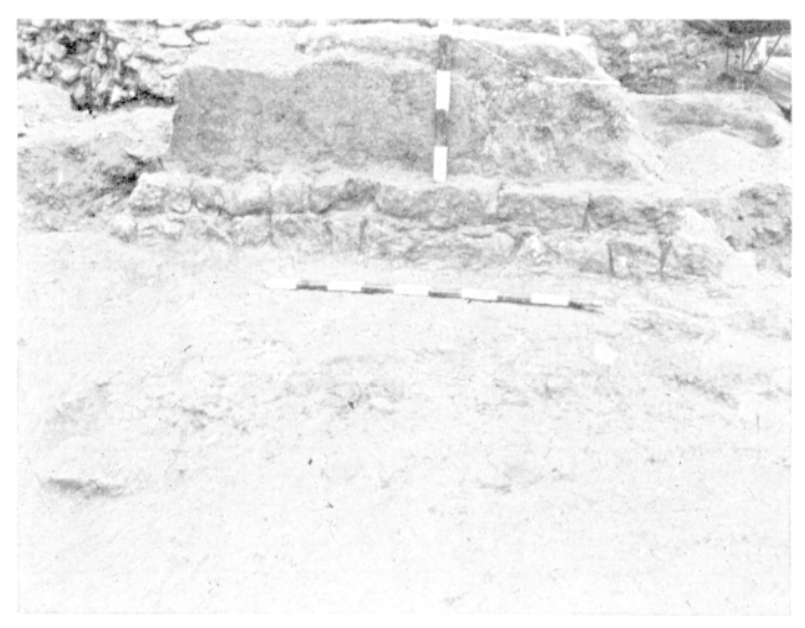

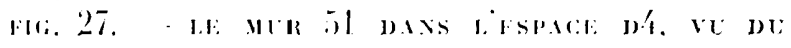

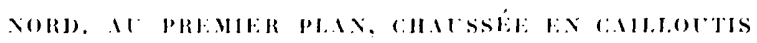
me " Decromen" guni, si aire betomene il y avait eu au nord, l'auraient-elles fait disparaitre entierement alors que de l'autre côte du mur, les restes de l'Antiguite, lien qu'rutames ici ou la, sont assez conservés pour rester lisibles?

Au demeurant, l'existence d'antimagements qui débordent aussi largement sur le domaine publie ne doit pas étomer

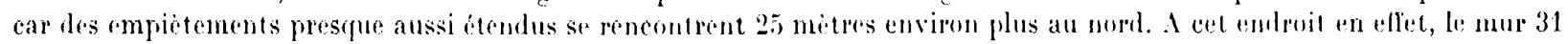

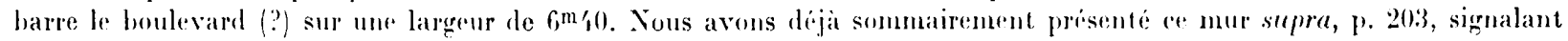

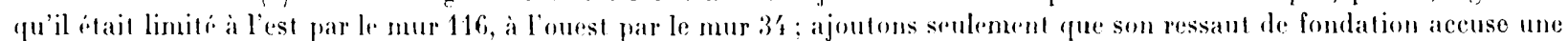

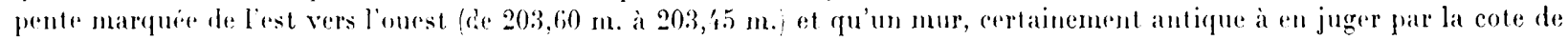

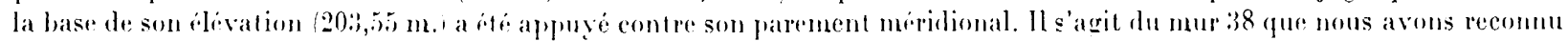
sur une longuener de 2 mètres seulement : c'est trop peu pour propreser une restitution. sulfisant pour ètre sûr que lon a elevé ici aussi, sur l'ancien boulevard l? a l'est du site, une autre construction eomplexe et itendur.

\section{b) Dotution.}

Lexhaussement des chanssées est bien daté par labondant materiel contenu dans les differents sols en terre battue et surtout les vastes fosses - dépotoirs sous-jacentes; mais a la cromique inventorié ri-dessous, il faudra ajouter le témoignage des momnaies.

le trait le plus marquant est que les fosses 8 a fournissent a elles seules plus de la moitie du matériel $51 \%$ o avec une forte proportion de fragments damphores plus de $60 \% \%$ : voir sur la figure 28.1 le bouchon $10^{\circ} 7797$, deux exemplaires entièrement reconstitues de fabrication regionale de la forme Dressel $2 / 4$ / 8972.8973 , on encore une amphore au profil curieux qui rappelle par certains traits la forme Dressel 20 5/190: fig. 28 B: ; la criramique commune à pâte brune est représentie par de nombreuses ollae (7891), celle à pâte claire par des cruches a une ou deux anses $8 / 12$ et 8/13! et la fouille a livré aussi son contingent d'objots estampillés ivoir infra, annexe IIj. Le facies des couches superposees $8 \mathrm{~b}$ iremblai d'aplanissement et 8 a sols en terre battue, parfois rendreux comme en Dyi n'est guère différent.

La datation est fournie par 150 tessons de ciramique sigillée claire 1 ides formes de la première génération, Lamb. 2 a, $1 / 36$ a feuilles deau et 10 1/10 B a strisce et les 18 tessons de sigillée claire B avec des formes Iamb. $8 /$ Darton 4', un couverele Desbat ' et un bord à gorge Desbat 66 ) qui conduisent à placer la refection des sols après les années 1/10-150 et ce jugement est pleinement confirmé par l'étude des sept momaies associés infra, anmexe numismatique nos $5,7,10$ à 13 et 17 qui vont d'un as posthume d'Auguste frappé apres 22 à un as posthume de Faustine I lik1-161), avee une forte concentration d'émissions sous le règne d'lladrien. 


\begin{tabular}{|c|c|c|c|c|c|c|c|c|c|c|c|c|c|c|c|c|c|c|c|c|c|c|}
\hline $\begin{array}{l}3 \\
y \\
0 \\
0 \\
j \\
0 \\
0 \\
0\end{array}$ & 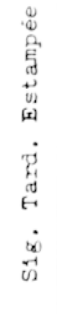 & $\begin{array}{l}A \\
\dot{u} \\
\dot{0} \\
\vec{\omega} \\
\dot{\omega}\end{array}$ & 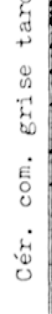 & 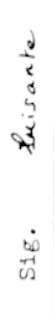 & $\begin{array}{l}m \\
\dot{u} \\
\dot{u} \\
\dot{\omega} \\
\dot{v}\end{array}$ & $\begin{array}{l}4 \\
\dot{u} \\
\dot{0} \\
\dot{\omega}\end{array}$ & 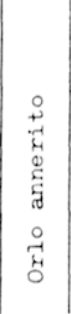 & 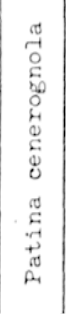 & 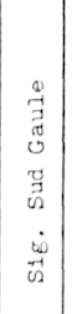 & 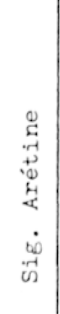 & 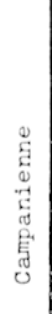 & 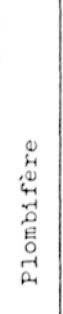 & 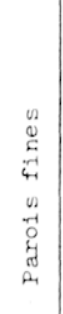 & 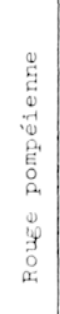 & 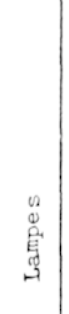 & 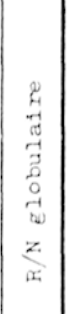 & $\begin{array}{c}0 \\
8 \\
8 \\
0 \\
0 \\
8 \\
0 \\
\dot{0} \\
0 \\
0 \\
\dot{8} \\
0 \\
0 \\
0\end{array}$ & $\begin{array}{l}0 \\
0 \\
0 \\
0 \\
6 \\
0 \\
\dot{E} \\
0 \\
0 \\
\dot{H} \\
0 \\
0\end{array}$ & 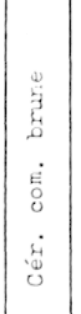 & 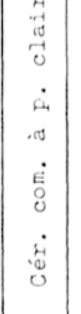 & 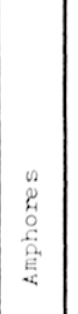 & $\begin{array}{l}\stackrel{\jmath}{d} \\
\stackrel{0}{0} \\
\stackrel{0}{0}\end{array}$ \\
\hline$i_{2} 80$ & & & & & 5 & 9 & 23 & 2 & $H$ & & & & & 3 & 2 & 1 & 21 & 10 & 124 & $i \sqrt[3]{3}$ & 122 & -1004 \\
\hline Id. $: b$ & & & & & 1 & 2 & & 8 & 6 & & & 1 & & & & & 6 & & 10 & 58 & 2,1 & $\mathrm{H}_{3}$ \\
\hline Id $\quad l_{c}$ & & & & & 3 & 12 & 1 & 10 & $\mu$ & & & 1 & 3 & 2 & 6 & 12 & 38 & $M$ & $\mu 7$ & 2,2 & $5 i 0$ & 491 \\
\hline$c_{3} \quad 8_{a}$ & & & & & 1 & 3 & 9 & 1 & $M$ & 3 & & & 5 & & 1 & 2 & 18 & 18 & 54 & $86^{\circ}$ & 84 & 246 \\
\hline Id. $\varepsilon_{b}$ & & & & & 4 & 48 & 21 & 17 & 45 & & & & 5 & & 14 & 53 & 75 & 38 & 348 & 461 & . $1:$ & $1: 45$ \\
\hline Id. $\quad c$ & & & & & 1 & 14 & 10 & 6 & 14 & & & 1 & 4 & 1 & 18 & 21 & 23 & $M$ & 236 & 280 & $33 \%$ & 922 \\
\hline$C_{-4} \quad 8$ & & & & & & 13 & 11 & 12 & 5 & & & & 1 & & 2 & 11 & 17 & 19 & :3 & 39 & 35 & 228 \\
\hline $2 d \quad 8 b$ & & & & & & 13 & 9 & 8 & 2 & & & & & & 1 & 30 & 28 & -11 & ig & 73 & 68 & 322 \\
\hline Id. $8 \mathrm{c}$ & & & & & 1 & 1 & & 2 & 1 & & & & & & & 1 & 9 & 3 & 31 & 26 & 73 & 147 \\
\hline c5 80 & & & & & 1 & 10 & 10 & 18 & 20 & & & 3 & 4 & 1 & & 5 & 46 & 22 & 81 & 186 & 1060 & 1467 \\
\hline$c_{6} \quad 8 b$ & & & & & & 12 & 6 & 4 & 12 & & & & 1 & 1 & 1 & 11 & 20 & 17 & 48 & $\mu_{4}$ & 86 & 383 \\
\hline Id. $i_{c}$ & & & & & & 7 & 4 & 4 & 3 & & & & & 1 & & 3 & 6 & 7 & 33 & 62 & 24 & $1 \sqrt{9}$ \\
\hline$C 7 \quad 8 c$ & & & & & & 4 & & 2 & 4 & 1 & & & 1 & & & 3 & 7 & 4 & 65 & 44 & $6: 2$ & 747 \\
\hline $\mathrm{O}_{2} \quad 8$ & & & & & & & & & 1 & 1 & & & $\lambda$ & & & & 8 & 3 & 2 & 6 & 1 & 23 \\
\hline $\begin{array}{ll}23 & 8 a \\
\end{array}$ & & & & & & 1 & 1 & & 99 & & & & 5 & 1 & 1 & 1 & 6 & 1 & 8 & 13 & 24 & 161 \\
\hline Id. $8 b$ & & & & & 1 & & & & & 1 & & & & 2 & 1 & & to & 7 & 13 & 3 & 9 & 47 \\
\hline DH 8 & & & & & & 1 & & 1 & 4 & & & & 1 & & 1 & 1 & 7 & 1 & in & 15 & 41 & 87 \\
\hline TOTAL & & & & & 18 & 150 & 111 & 95 & 242 & 6 & & 6 & 31 & 12 & 43 & 155 & 345 & 183 & 1376 & 2,341 & $35: 2$ & 8647 \\
\hline
\end{tabular}

Tabliau $V$. Nomenclature de la céramique provenant des couches de recharge des chaussées, jusqu'à la cote $203,55 / 203,80 \mathrm{~m}$.

Les indices chronologiques sont très voisins de ceux que nous avons relevés sous les bétons de l'insula I et il n'y a pas lieu de s'en étonner : la réfection des chaussées aurait accompagné (ou suivi de peu?) l'importante restauration du bâtiment voisin. Plus important pour notre propos est de remarquer que certains au moins des empiétements sur le domaine public sont en revanche postérieurs : en C.3, les fondations d'un élément du muret 36 ont en effet entamé l'une des fosses placées sous la chaussée et le blocage garde encore l'empreinte des amphores contre lesquelles il a été coulé (fig. 29). Notons pourtant que l'indice vaut pour le seul mur 36 et non pour l'ensemble de l'alignement auquel il participe car les murets ont pu être appuyés dans un second temps sur les dés de fondation placés devant la façade est de l'insula I; en revanche, l'indice est important pour interpréter les indications chronologiques fournies par l'étude du matériel recueilli sous les niveaux chaulés qui s'étendent continûment entre les murs 30 et 36 .

Le lecteur se souvient qu'il s'agit de deux niveaux superposés, également installés en contrebas de la chaussće après excavation de son revêtement. Le niveau inférieur est à la cote $203,47 \mathrm{~m}$. et le tableau suivant donne la nomenclature de son matériel céramique. 


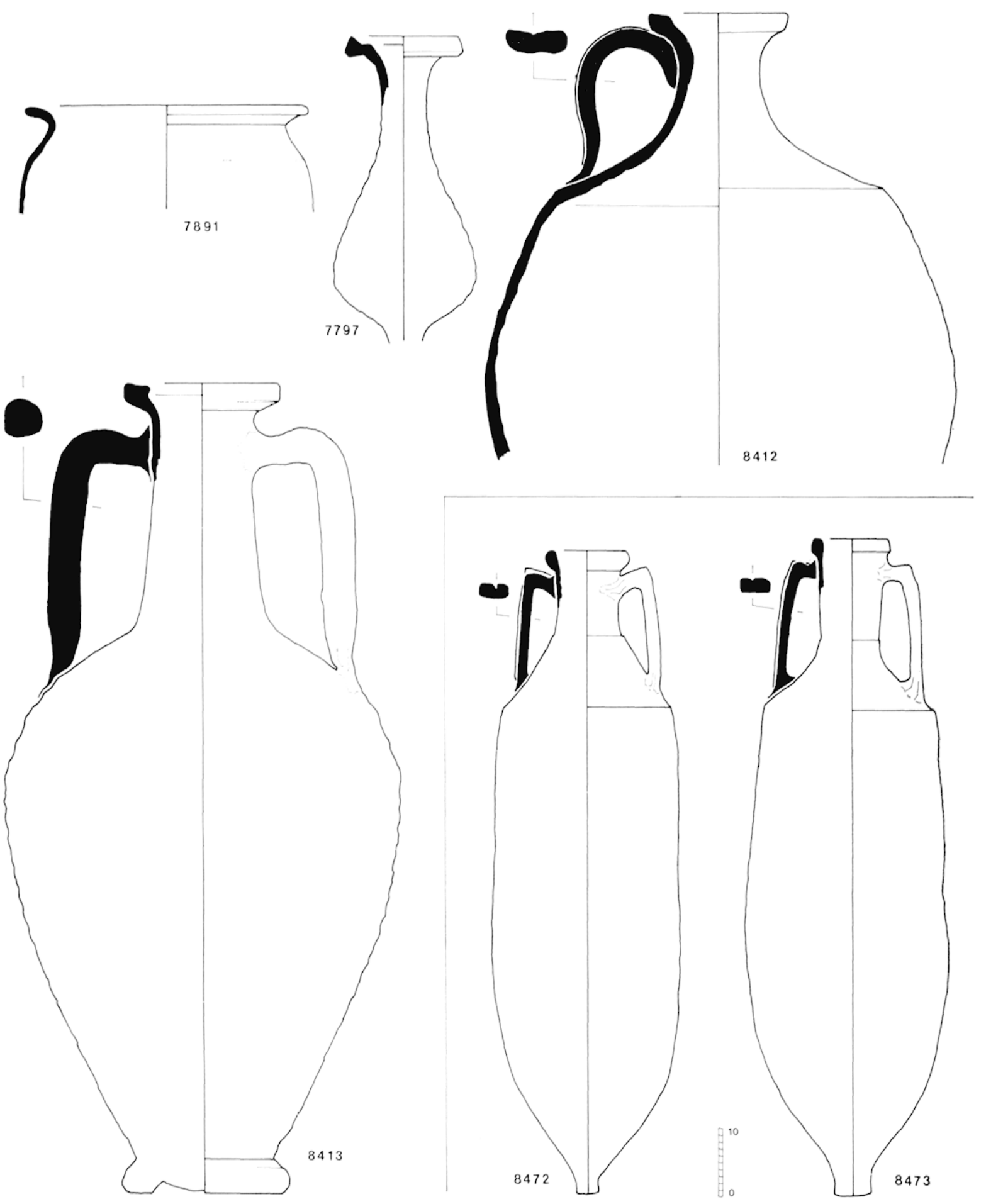

fig. 28 a. - Matériel céramique provexant des couches de recharge drs chaussétes ÉCHANTILLONNAGE DES FORMES

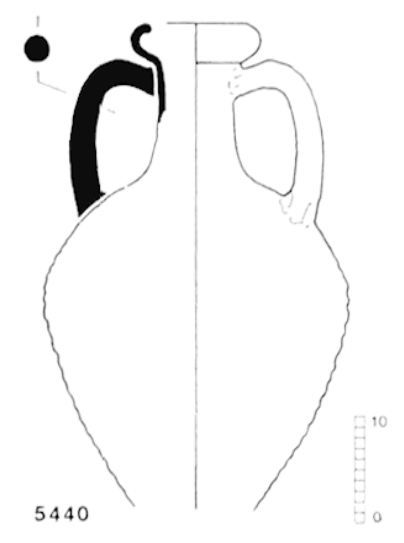

FIG. 28 B. - AMPHORE De fabrication hocalf, RAPPELANT TA FORME DRESSEL 20, APPARTENANT AU COMBLEMENT D'UNE FOSSE DE LA CHAUSSÉE 


\begin{tabular}{|c|c|c|c|c|c|c|c|c|c|c|c|c|c|c|c|c|c|c|c|c|c|c|}
\hline $\begin{array}{ll}u & y \\
w & y \\
u & w \\
\alpha & y \\
a & y \\
y & j \\
w & 0 \\
& u \\
\end{array}$ & 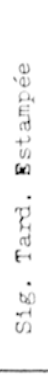 & $\begin{array}{l}\text { A } \\
\dot{u} \\
\dot{u} \\
\dot{u} \\
\dot{u}\end{array}$ & 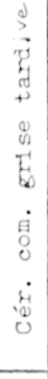 & के & $\begin{array}{l}m \\
\dot{u} \\
\dot{u} \\
\dot{\omega} \\
\vec{u}\end{array}$ & $\begin{array}{l}\varangle \\
\dot{u} \\
\dot{0} \\
\dot{0} \\
\text { of }\end{array}$ & \begin{tabular}{l}
0 \\
+ \\
\multirow{4}{4}{} \\
0 \\
5 \\
तु \\
0 \\
के \\
मे
\end{tabular} & 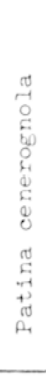 & 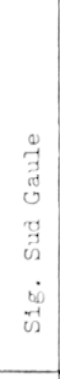 & 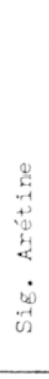 & 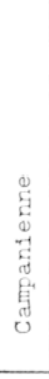 & 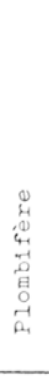 & 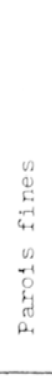 & 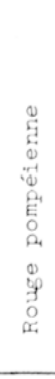 & 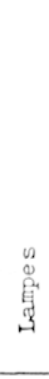 & 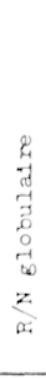 & $\begin{array}{l}0 \\
8 \\
0 \\
0 \\
8 \\
0 \\
8 \\
0 \\
0 \\
0 \\
\dot{0} \\
0 \\
0\end{array}$ & $\begin{array}{l}\mathscr{8} \\
\overrightarrow{6} \\
\dot{0} \\
\dot{E} \\
0 \\
0 \\
\dot{\phi} \\
\dot{0}\end{array}$ & $\begin{array}{l}\text { D } \\
\text { 胳 } \\
\dot{E} \\
\dot{0} \\
0 \\
\dot{0} \\
\dot{0}\end{array}$ & 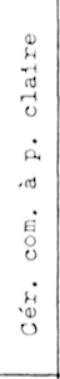 & 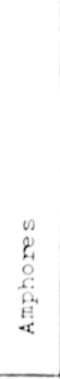 & $\begin{array}{l}\overrightarrow{0} \\
\stackrel{0}{0} \\
0 \\
0\end{array}$ \\
\hline$P_{3} \cdot P_{4} \quad 8_{C}$ & & & & & & & 1 & & 2 & & & & $\lambda$ & $\Lambda$ & $\lambda$ & $\Lambda$ & 8 & 2 & 42 & 52 & $\bar{i}$ & 202 \\
\hline Id $\quad 8 d$ & & & & & & & & & 12 & & & & 2 & 1 & & & 4 & 4 & 7 & 28 & 33 & 91 \\
\hline Id. $8 e$ & & & & & & & & & & & & & & & & & 2 & & 139 & 3 & H & 148 \\
\hline is ic & & & & & & 1 & & 1 & 4 & & & & $\lambda$ & & & & 7 & 11 & 20 & 21 & 3.9 & 105 \\
\hline Id. $\quad 8 d$ & & & & & & & & & 5 & & & & 1 & & & & 10 & & H & 9 & 27 & 56 \\
\hline$P_{7} \quad$ red & & & & & & & & & 3 & & & & & & & & 3 & & 6 & 8 & 20 & 40 \\
\hline TOTAL & & & & & & 1 & 1 & 1 & 36 & & & & 5 & 2 & 1 & 1 & 344 & if & 218 & 121 & 204 & 642 \\
\hline
\end{tabular}

'Tablea U VI. Nomenclature de la réramique provenant des couches placées au-dessous du premier niveau de circulation du portique.

I tes strates 8 c correspondent à l'assise du sol tandis que les couches $8 \mathrm{~d}$ sont des recharges ou des fosses sous-jacentes et 8 e, le foyer déja signalé, supra, p. 205, qui a entamé le sol en caillout is de la chaussée de la fin du ${ }_{1}^{\text {er }}$ siècle mais est secllé par le niveau chaulé. Ce foyer est important car il recèle une monnaie émise en 85 infra, annexe numismatique, no 9 qui fournit un indice de datation sensiblement postérieur à ceux que suggère l'étude des seules ceramiques datables : cinq fragments de sigillée du sud de la Craule répondant a des formes produites a partir de Néron et un unique tesson de sigillée claire 1 (de la forme précoce Lamb. 10 Aj qui ne peut être antérieur aux années 60-70. Le remblai est cependant sùrement très postérieur an règne de Domitien puisqu il est appuyé contre le mur 36 dont nous avons place la construction après le milieu du ne siècle : nouvel exemple d'une discordance entre données céramologiques, numismatiques et architecturales dont l'étude du niveau sus-jacent offrira encore une illustration.

La recharge chaulée constituéc à la cote $203,58 \mathrm{~m}$. (couche 8 a) et les fosses ou remblais inférieurs (8 b) contiennent en effet un matériel différent selon les secteurs comme le montre le tableau suivant.

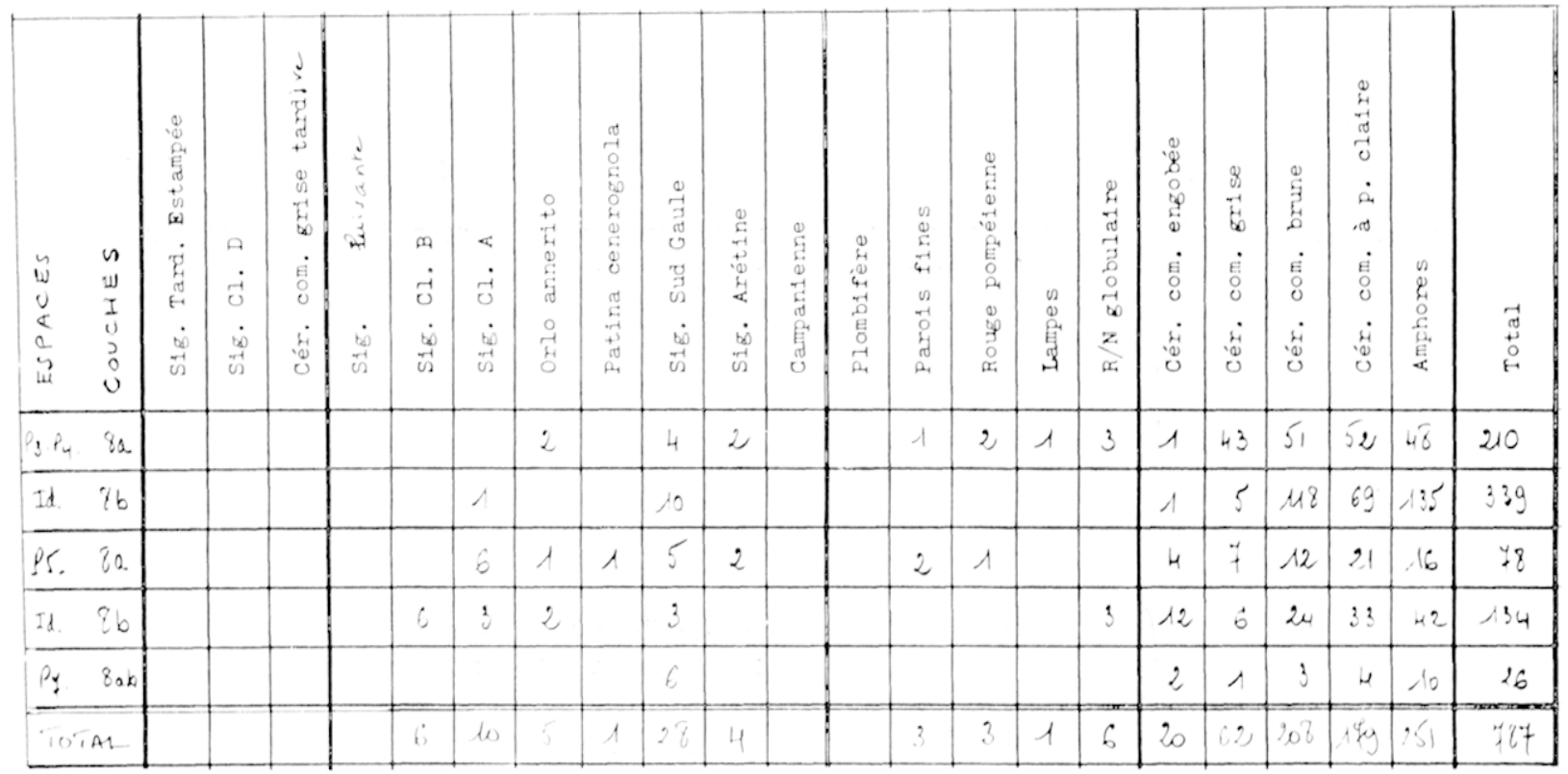

Támilu VII. Nomenclature de la céramique provenant des couches placées au-dessous du second niveau de circulation du portique. 


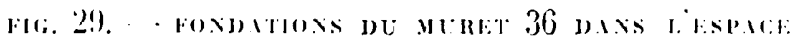

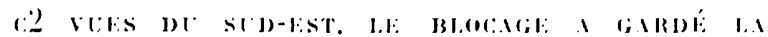

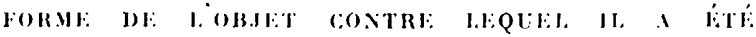

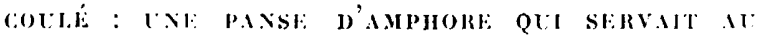

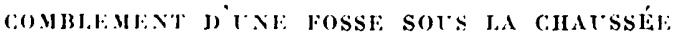

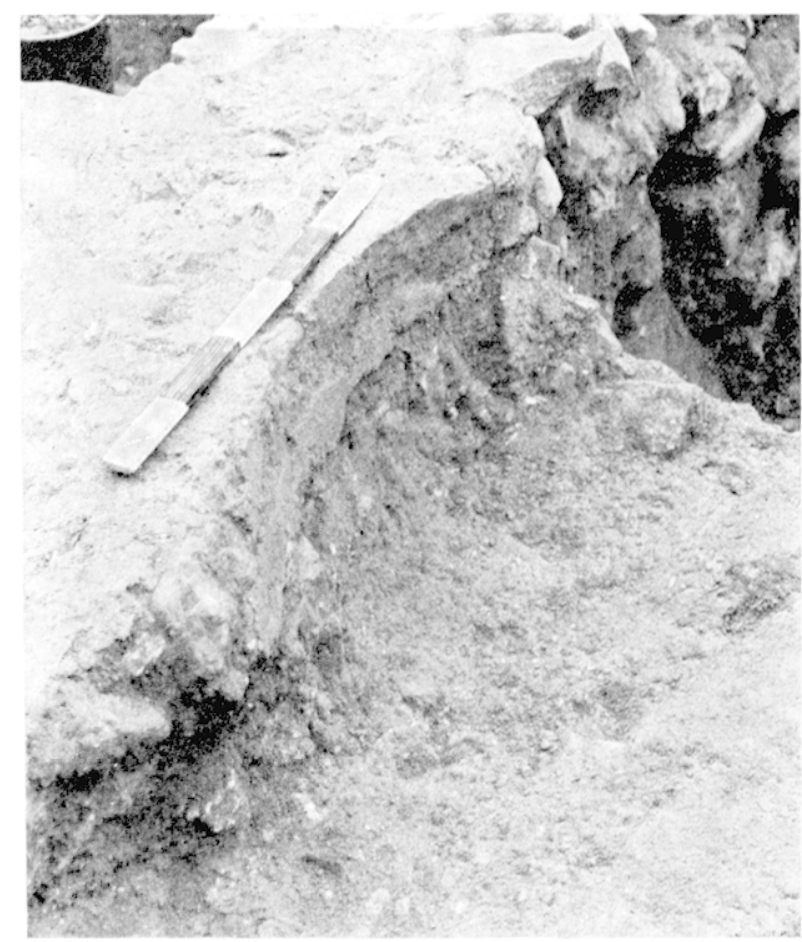

I considerer la plupart des espaces, on retronve un faciès comparable à celui du niveau inférieur, avec de la sigillé du sud de la Gaule produite sous les règnes de Tibère et Claude, mais la présence dans l'espace P5 de six fragments d iune forme ferme de sigillée claire 3 produite après 140-150 et l'existence dans les remblais du secteur P7 d'une monnaie émise en 166 n० 20 de lannexo mumismatique) sullisent dans le cas present à repousser la datation du remblai a une date compatible avec celle de la construction du muret contre lequel il a été appuyé ; nul doute cependant que cette fois encore, la couche est plus récente que ne l'indique le matériel puisqu'elle répond à une recharge et non au premier niveau du sol associé au mur 36.

\section{c) Restilution.}

C'est sans nul doute à l'est de l'insula I que se trouvent le's éléments les plus clairs pour une restitution : les des massifs en maçonnerie servaient certainement d'appuis aux supports d'une couverture, poteaux, pilastres ou colonnes et les différents éléments du nur 36 constituaient un mur bahut qui séparait du charroi de la chaussée voisine un cheminement piéton sous le couvert d'un portique. Comme la situation est tres comparable au long de la façade nord de l'insula II où se rencontrent non seulement des sols identiques à la fois par leur cote, leur facture et leur datation mais aussi des dés de fondation au sein de l'appareil du mur 51, il faut sans doute imaginer à cet endroit, après le milieu du ${ }_{11}{ }^{\mathrm{e}}$ siècle, un second portique, d'ailleurs en relation avec le précédent à hauteur de l'espace Pó.

La restitution de ces portiques implique naturellement la condamnation de la chaussée du decumanus fig. 30) mais elle ne gêne en rien la vie au scin de l'insula I; au contraire, clle la faciliterait plutôt. $\Lambda$ l'est en effet où les exhaussements et les réfections avaient porté le niveau de la chaussée au-dessus de celui des pièces de toute la partie orientale du bâtiment, le sol du portique, même une fois rechargé, reste désormais en controbas de la chaussée et de l’insula tout à la fois; sur la façade méridionale de même, l'accessibilité des seuils est préservée a cause du niveau de circulation assez bas qui a été maintenu au nord du mur jl tandis qu'au sud de cet ouvrage, la circulation pietonne est en continuité avec celle de la façade est.

Aussi n'est-il pas itomnant que le bâtiment soit sans doute resté longtemps en fonction comme le montrera la suite de cette étude; les traces d'une quelconque évolution y sont même minimes : tout au plus peut-on supposer que les pièces au nord de la cour ont été mal entretenues ou peut-être même abandonnées) avant le début de l’Antiquité tardive. L’impression se fonde sur la découverte dans les salles $\mathrm{\Lambda L}$ et $\mathrm{\Lambda}$ d'un tas de cendres istérile, mais contenant de nombreuses coulures de plomb fondu qui témoignent d'une activité de récupérationj et sur la présence a proximité d'un tas de terre rougeâtre qui contenait 28 fragments de céramique sigillée claire C. appartenant à deux objets seulement formes Lamb. 35 et 40 ), déjà brisés et qui avaient été abandonnés sur place ; l'association 
à ce dépôt d'un fragment de céramique sigillée claire $\mathrm{D}$ oblige à le dater au plus tôt de la fin du $n^{\mathrm{e}}$ ou du début $\mathrm{du} \mathrm{Iv}^{\mathrm{e}}$ siècle. Nous ne disposons pas de renseignements comparables à propos de l'insula II; notons seulement que le niveau du portique est cette fois supérieur à celui des sols en béton de l'édifice : ce peut être l'indice que le bâtiment comptait peu ou pas d'ouvertures vers le nord (44).

La restitution de portiques sur les rues est donc cohérente et ne se heurte à aucune difficulté majeure. Lille conduit pourtant à réunir dans un même ensemble des éléments assez hétérogènes d'aspect comme le mur 51 et les murets 36 ; aussi est-il presque sûr que la situation que nous avons décrite résulte, non d'une unique campagne de travaux mais de toute une évolution que nous pouvons seulement imaginer.

Nous croirions volontiers que les portiques ont initialement bordé les seules façades des insulae. A l'est de l'insula I, le dispositif très simple que nous restituons ief. fig. 30 s'accommoderait bien du voisinage des installations artisanales et commerciales dont nous avons supposé l'existence dans tout le secteur oriental de l'édifice : espacement irrégulier des supports, médiocrité du mur bahut qui a pu n'être élevé d'ailleurs que dans un second temps notons par parenthèse que si cette supposition se rérélait exacte, l'érection du portique serait probablement antérieure à la dernière restauration de l'insula I que nous avons présentée supra, p. 210-220) (45).

La faģade nord de l'insula II aurait été bordée également d'un portique qui pouvait faire retour à l'origine sur le flanc est du bâtiment. Comme nous l'avons déjà supposé supra, p. 209, le mur 12 marquait peut-être en effet la limite orientale de l'insula de la fin du ser siècle et le dé placé à l'angle du bâtiment achevé pourrait donc être l'unique témoin conservé

FIG. 30. - - vel: Axoxométrique Restitcée de secTEUR SUD-EST DU SITE (COMPARER AVE: ia FIG. 23) (DESSIX : J. BIGOT) d'un portique ultérieurement transformé en salle, L: Hypothèse sans doute, mais qu'il est possible - pour une fois dans l'insula II ... d'ètayer quelque peu par les données de la fouille. Contre le mur 46 qui marque la limite orientale de l'insula achevée et sous le sol en béton de l'espace U subsiste en effet un mur dont l'arasement témoigne à coup sûr d'un remaniement important : peut-être le mur bahut du portique que nous venons d'évoquer (46)?

Après le milieu du ${ }_{11}^{\mathrm{e}}$ siècle en tout cas, ces différents portiques ont sûrement été réunis pour former l'ensemble représenté sur les figures 24 et 30 . Leur réunion interdit tout charroi sur le decumanus, mais pas nécessairement sur le boulevard (?) à l'est du site que ces premiers empiétements ont à peine restreint ; à examiner l'aspect soigné de la chaussée du boulevard, il est même presque certain qu'elle servait toujours alors à la circulation. C'est pourquoi nous jugerions encore postérieures les dernières constructions sur l'espace ouvert (en $R$ et au nord de C1) qu'il est impossible de dater de façon satisfaisante (47).

En présentant cette reconstitution même comme une simple hypothèse, nous sommes conscients de trop céder peut-être à une vision idéalisée de l'Antiquité qui nous pousse à retarder le plus possible le lotissement des rues et l'accaparement du domaine public. Ce lotissement et cet accaparement ont pourtant existé et au terme d'une évolution dont la chronologie précise nous échappe, l'urbanisme réglé de la fin du ${ }^{\text {er }}$ siècle a fait place dans le secteur à une situation beaucoup plus confuse.

Le trait ne doit pas surprendre car dès qu'elles sont menées avec assez de soin, les fouilles urlaines montrent que l'histoire de la voirie est faite d'empiétements successils qui conduisent à terme à l'abandon des rues. Dans le cas présent, cet abandon se comprend d'ailleurs assez bien : dès la fin du ${ }^{\text {er }}$ siècle, la masse imposante des monuments publics du forum voisin avait comme isolé du reste du réseau de circulation d'Aquae Sextiae la portion de decumanus présente sur le site et sans doute provoqué l'asphyxie de tout ce secteur de la ville. Iin quelques générations, les propriétaires riverains ont tiré profit de cette situation, multipliant les constructions adventices qui ont gravement altéré le décor, puis faisant leur la majeure partie de rues qui ne servaient plus guère à la collectivité. La majeure partie, mais non la totalité : un passage pour les piétons restait possible -- et ce jusque pendant l'Antiquité tardive et le haut Moyen Age. 


\section{1. - Les permanexces de a'Antiquté tandive et de hate Moyen AgF}

De nouveaux exhaussements du sol et peut-être un nouveau mur à l'est de l'insula I ; une fosse à l'intérieur du bâtiment et une tombe ì proximité de l'insula $\mathrm{II}$ : tels sont les seuls témoignages assurés de l'Antiquité tardive sur le site. Cela ne veut pas dire pour autant que tout ait éti alors à l'abandon ;'examen des immeubles invite au contraire à placer sous le signe de la permanence l'étude de cette période essentielle de l'histoire du secteur (et surtout du quartier alentour).

a) Les vestiges de l'Antiquité tardive : description et datation.

I l'est de l'insula I, la recherche a mis en évidence de nouvelles recharges qui semblent avoir porté vers 203,93 m. Le niveau du sol, non seulement sur le boulevard rertainement condamné désormais, mais aussi sous le couvert du portique. Les remaniements médiévaux avaient fortement perturbé ce niveau qui n'a été fouillé qu'en quelques endroits seulement.

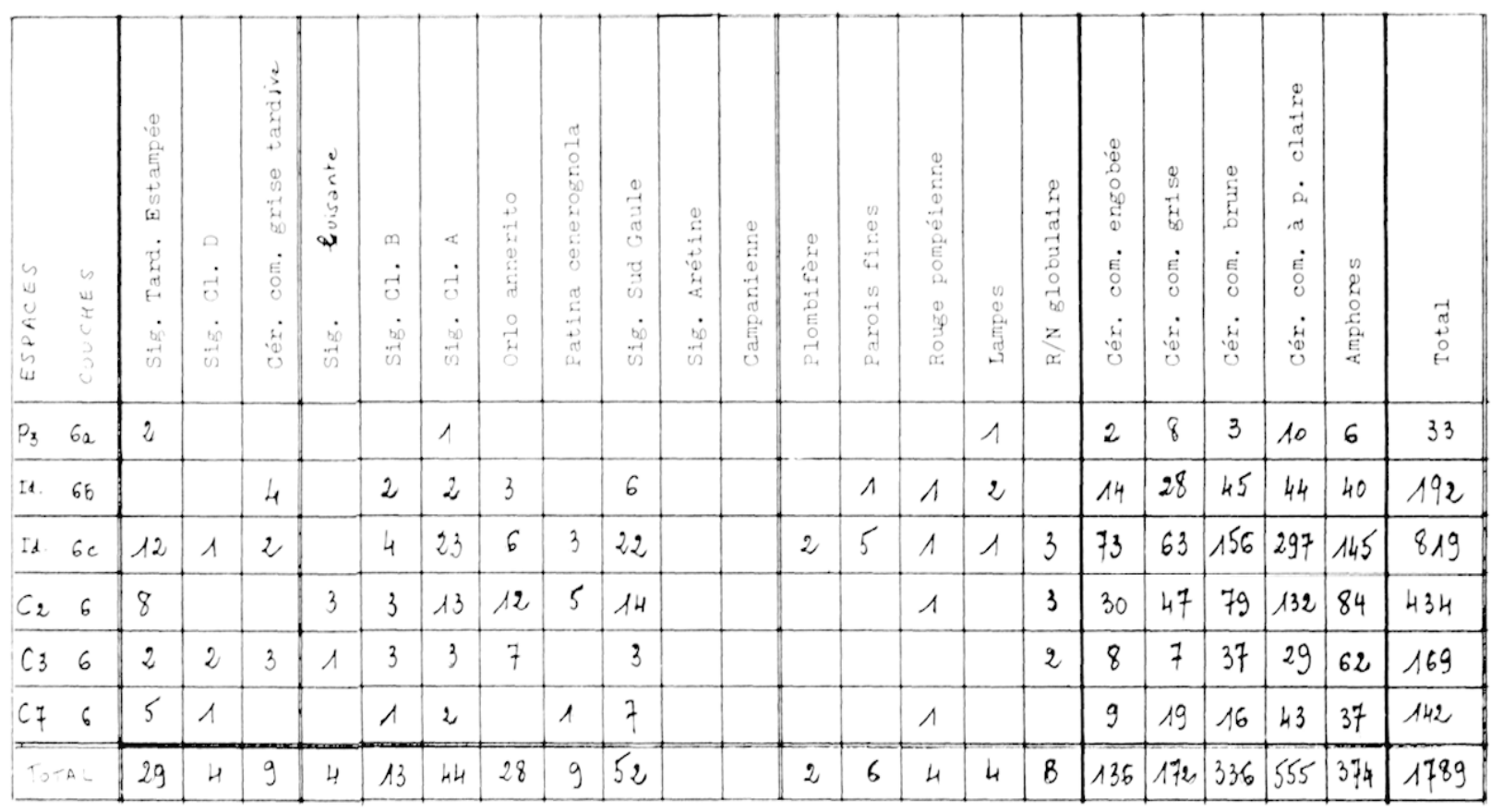

'I'a briau VIII. Nomenclature de la céramique des couches de recharge des chaussées (jusqu'à la cote 203,93 m.).

Le tableau ci-dessus fournit la nomenclature de la céramique recueillie : l'essentiel est constitué par des tessons qui appartienment à l'Antiquité classique mais ils sont associés à 29 fragments de sigillée tardive estampée et surtout à un rebord de céramique sigillée claire D de la forme Hayes $73 \mathrm{~A}$ dont la seule présence suffit à montrer que le niveau a été constitué ou refait après les premières décennies du ve siècle.

Dans la même zone, nous avons également repéré un muret, 52, qui marque la césure entre les espaces C.3 et C6. Il est médiocrement conservé sur une longueur d'un mètre à peine mais on a bien retrouvé, au moins en direction du sud, la tranchée de récupération de ses matériaux et il faut sans doute le relier plus au nord à un élément de mur (qui apparaissait en limite nord du premier secteur que nous avons fouille, en C.7 (cf. fig. 26). Le mur 52 aurait donc mesuré au moins 8 mètres de long, en présentant une direction légèrement oblique par rapport a celle du mur 36. Fin l'absence de tout élément de datation, c'est le niveau très superficiel de la base des fondations $1203, j 7 \mathrm{~m}$. au nord, 203, $77 \mathrm{~m}$. au sud) qui nous conduit à attribuer, sous toutes réserves, à l'Antiquité tardive ce mur qui ne semble d'ailleurs avoir joué aucun rôle dans les constructions médiévales ultérieures.

Dans l'insula I en revanche, la fouille de l'espace I, a livré dans le matériel de comblement d'une grande fosse de sûrs vestiges de l'Intiquité tardive. 


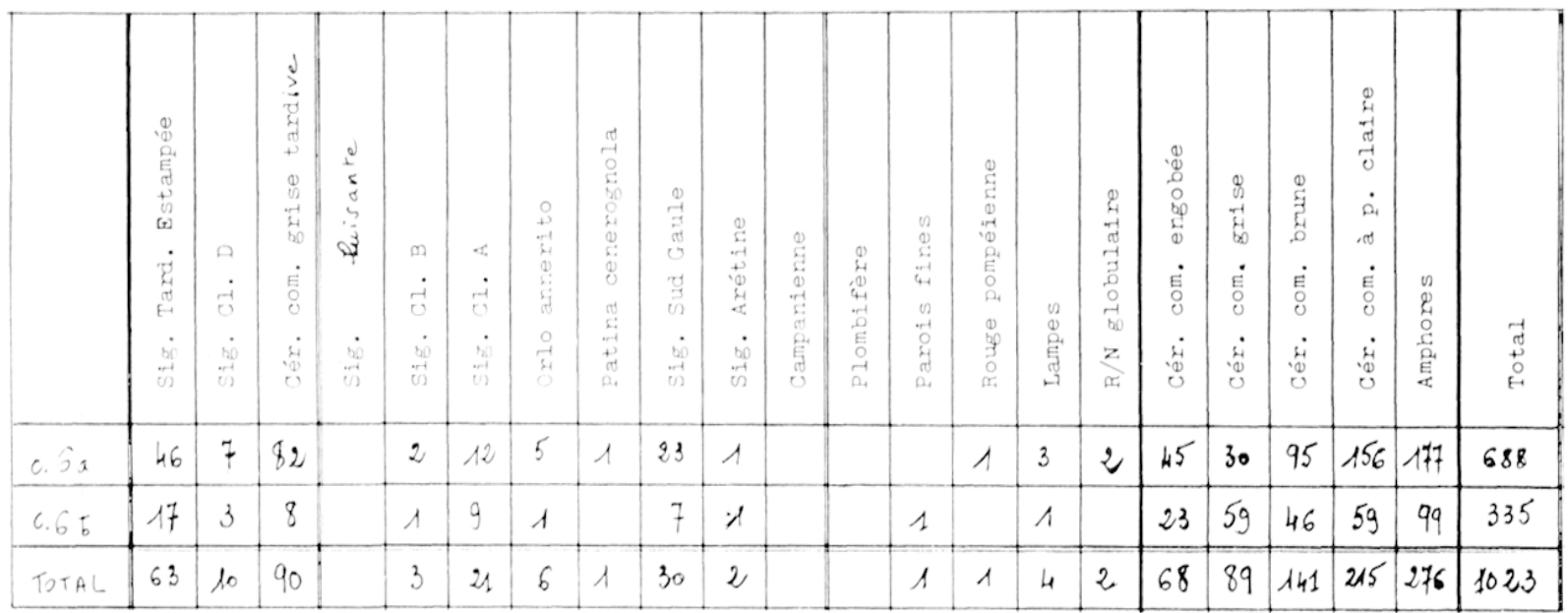

Tabiene IX. Nomenelature de la céramique provenant du comblement de la fosse de la pièce L.

Comme le montre le tableau ci-dessus, où la couche 6 a correspond a la fosse et 6 b à la couche encaissante, les fragments de sigillee estampe tardive lig. 3L sont particulièrement nombreux ot l'on notera, a côté d'une vaisselle du groupe provenģal provenant très probablement des ateliers marseillais, d'autres pièces très caracteristiques de la production languedocienne : un col de forme Rig. 18 guilloché et souligné do rainures 'nº 12 ' 186 , un bord de forme Rig. 6 décore de palmettes 1.3320 et un haut de bord en pâte groscière dont le décor est constitué de rectangles composés de chevrons 125ls; ; cependant, les fragments informes ou swulement décorés dr motifs simples de la céramique sigillée claire $D$ associće à ce matériel ne permettent pas de dater précisément le comblement de la fosse.

A l'est de l'insula II enfin, a été retrouvée une tombe sous tuiles d'ailleurs très remaniée et que les conditions très difficiles de la recherche dans l'expace (a) ont conduit à reconnaître très rapidement. La fouille n'a fourni aucun élément de datation et il serait imprudent de proposer une chronologie trop précise a l'aide de la seule typologie car les tombes en bâtière ont peut-être ité en usage plus longtemps qu'on ne le pense généralement, au moins dans les régions méridionales; il reste qu'une telle inhumation appartient eertainement à l'Antiquité tardive ou au haut Moyen Age.
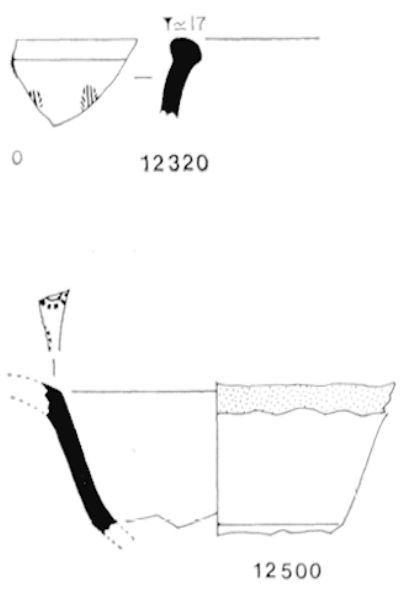
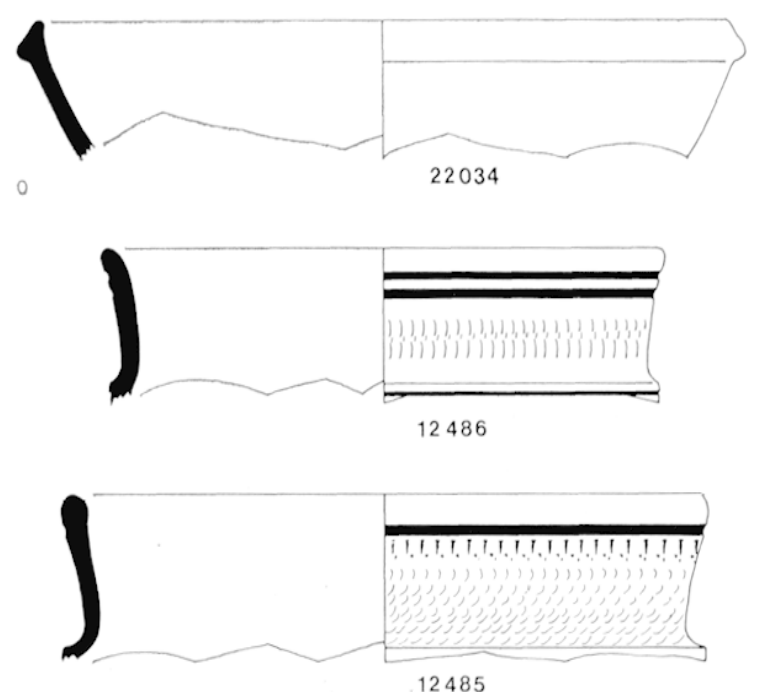
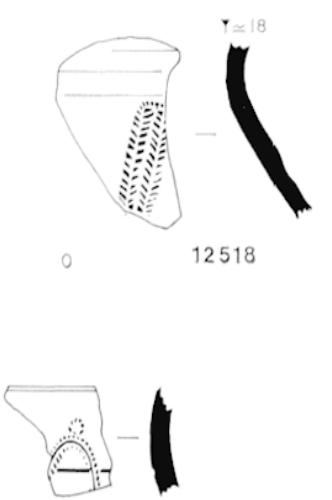

12491

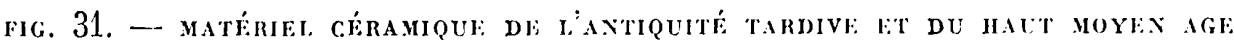

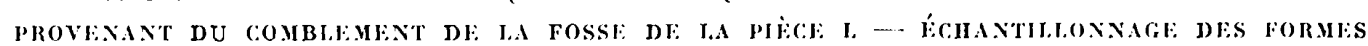

12320 : bord de f. 6 proche des productions languedociennes; 22034 : bord de f. 8 profonde ; 12518 : fragment de panse (de bol?), fabrication rustique; 12486 : col de f. 18 assez caractéristique de la production de Marseille par le profil et par les guillochis; 12485 : col de f. 18 de style different, non engobé, grossièrement lustré à l'intérieur ; 12500 : fragment de bol à marli ; la rectitude de la panse carćnće fait penser à la f. 46 ; forme rare ; 12491 : fragment de panse (f. 18?); pâte rougeâtre qui se retrouve sur des pièces de Marseille; arceau répertorié dans la même région. 


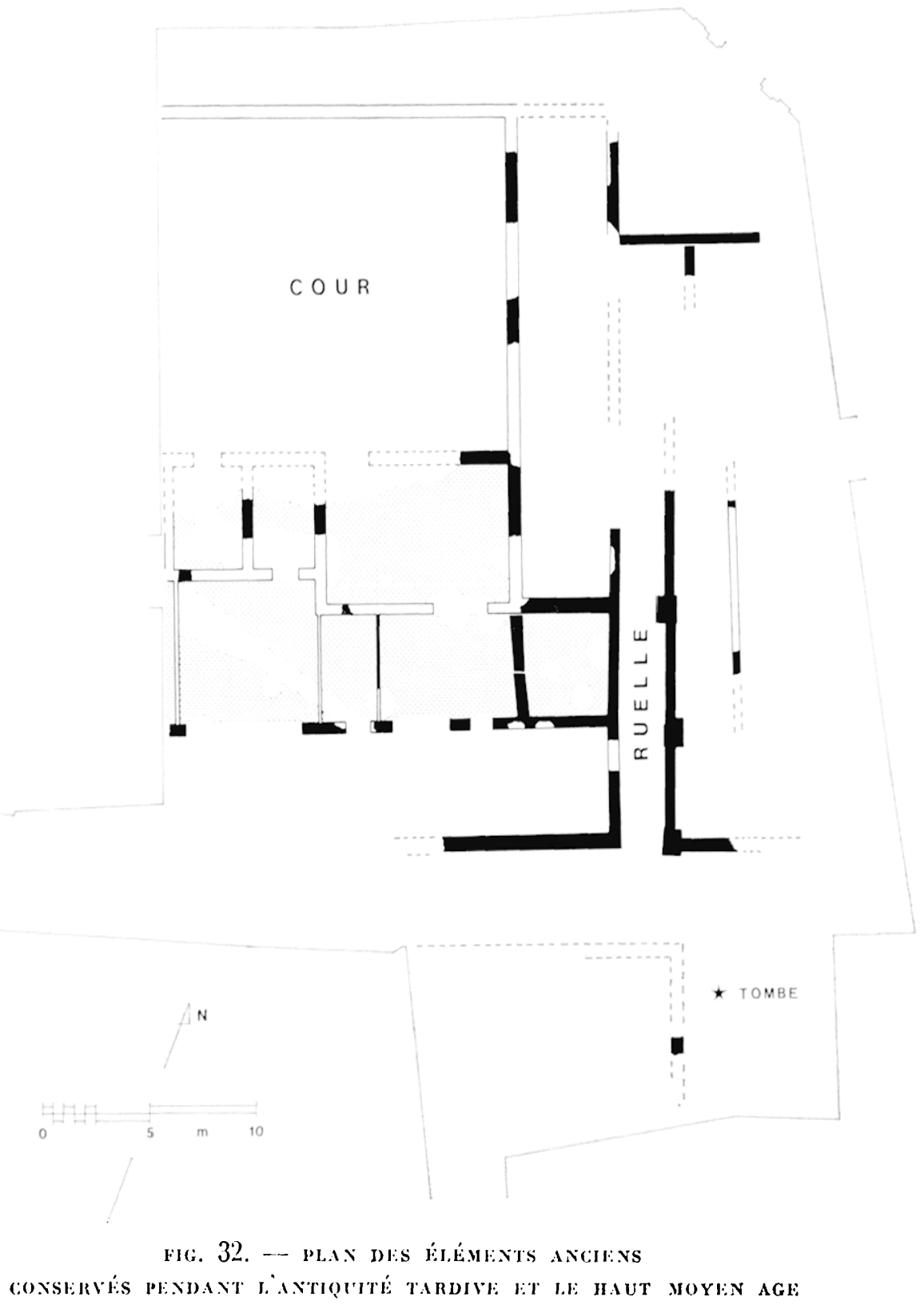

b) La conservation des bâtiments antiques.

A côté de ces sûrs témoignages de l'activité humaine pendant l'Intiquité tardive, il faut compter aussi avec la conservation des bâtiments antiques. La suite de cette étude montrera en effet combien l'architecture du palais archiépiscopal est restée tributaire de l'urbanisme antique pendant presque toute la durie du Mloyen $\Lambda$ ge et les indices sont done suffisants pour repérer quels éléments en ont été conservés, sûrement ou vraisemblablement, pendant toute l'Antiquité tardive et le haut MLoyen Ige : voir sur 'ee point la figure 32 sur laquelle les conventions graphiques indiquent, du gras au simple pointillé, le degré de probabilité de notre restitution.

En noir ont été représentés les murs qui journt certainement un ròle au Moyen Age et parfois jusque dans un Moyen Age avancé) ; en blanc figure d'autre part le mur 4.3 dont l'alignement a persisté pendant une partie du Moyen Age et dont les matériaux n'ont dailleurs été récupérés que tardivement comme en témoigne la présence de céramique vernissée au sein de la tranchée qui nous en a conservé la trace; nous avons représenté enfin en pointillés les contours de l'insula II parce que nous ne disposons pas pour cet édifice de renseignements assez précis, mais le peu que l'on entrevoit ne diffère guère de la situation rencontrée plus an nord : les sols en béton sont aussi bien conservés que dans l'insula I "t surtout des murs médiévaux ou modernes ont été construits ici enrore au- 


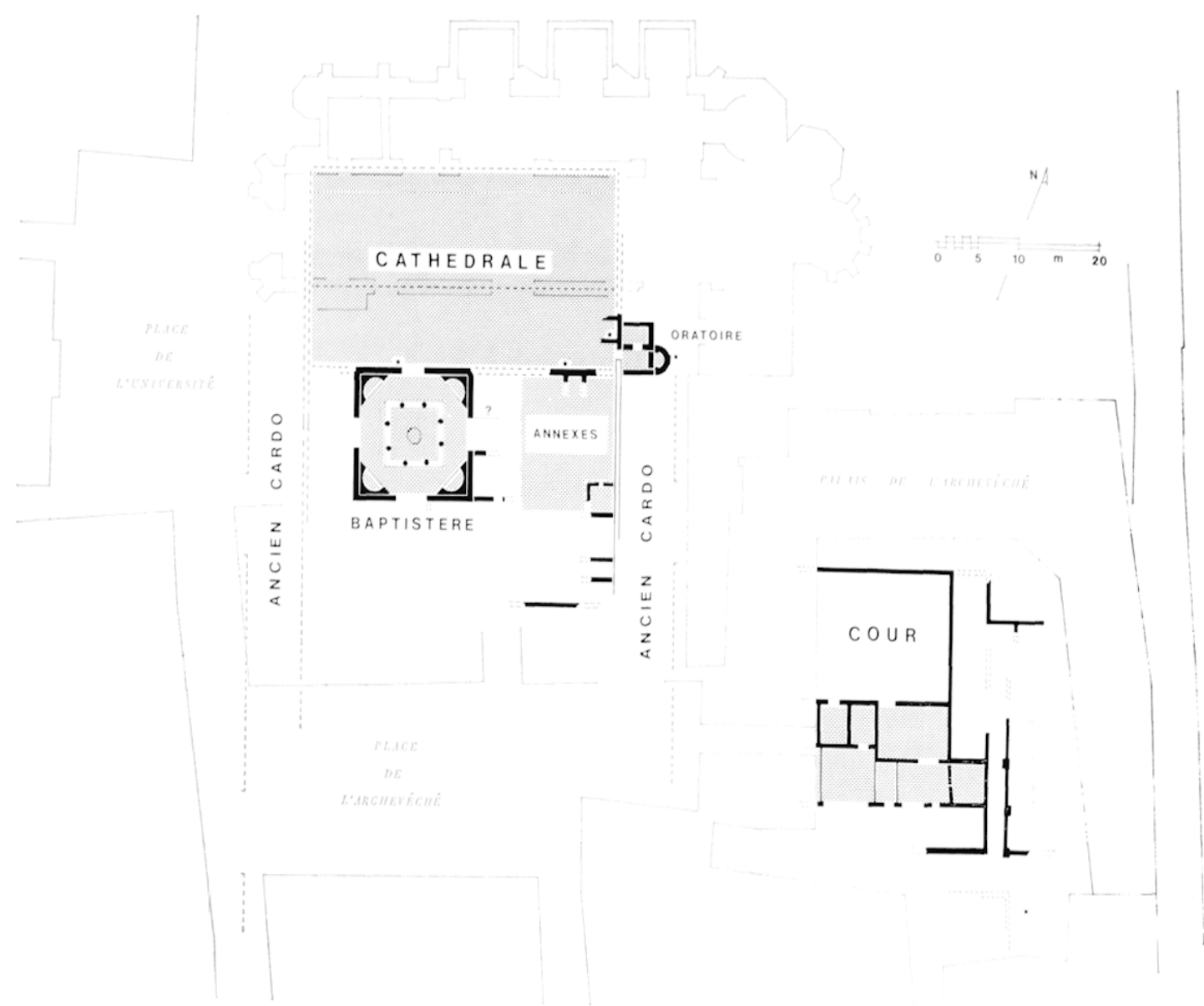

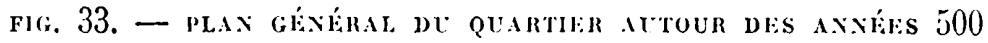

I.ES TOMBES SONT INDIQVÉLS PAR DES ÉTOILES

dessus d'élévations antiques (mur des écuries au-dessus du mur $5 \overline{7}$, mur moderne au-dessus du mur 16), ce qui peut laisser supposer que le bâtiment a été aussi largement conservé que l'insula I.

Il suffit en effet de comparer les schémas des figures $2 / 4$ et 32 pour constater que l'essentiel de l'insula I (et même des constructions adjacentes sur le cardo) a été préservé pendant l'Antiquité tardive et le haut Moyen Age; encore l'évolution que suggère le rapprochement de ces deux documents était-elle peut-être moins marquée en réalité car les éléments que nous avons reportés sur la figure 32 sont les seuls dont l'existence nous parait assurée et les importantes lacunes qui apparaissent désormais autour de la cour ou dans le secteur oriental de l'insula antique peuvent tenir aux insuffisances de notre documentation $48 ;$ ou a des remaniements tardifs plus radicaux qu'ailleurs.

Il reste que nous avons pu tout au plus repérer quelques pans de murs ou quelques sols bien conservés et qu'une restitution précise serait aventureuse; il est possible en revanche d'imaginer au moins l'aspect général du site à la fin de l'Antiquité ou au début du Moyen Age.

c) Le quartier pendant le haut Moyen Age.

Nous pouvons prendre une vision d'ensemble du çur même d'Aquae Sextiae autour des années 500 grâce au schéma de la figure 33 qui associe le relevé des vestiges de la Cour de l'Archevêché et celui des fouilles récentes dans le groupe cathédral Saint-Sauveur. lin rapprochant ce dessin de celui de la figure 13, ce n'est plus le sentiment d'une permanence qui l'emporte cette fois, mais bien celui d'un véritable bouleversement de la topographie; au début du $\mathrm{vi}^{\mathrm{e}}$ siècle, le forum a disparu sous un important remblai qui a porté l'ensemble de la place publique au niveau du grand monument qui le dominait autrefois au nord (49) et sur ce monument a été construite une vaste cathédrale peut être composée de deux nefs accolées $(50)$ et d'ailleurs flanquée au sud-ouest par un grand baptistère (installé sur l'angle de l'ancien forumi et au sud-est par d'importants locaux annexes. Rien de comparable sous la Cour de l'Irchevêché, hormis la présence de la tombe près de l'insula II qu'il faut placer dans la même série que 
les sépultures analogues retrouvées à l'intérieur et alentour du groupe épiscopal : n’était ce rapprochement, sur le seul vu des fouilles du site, on inaginerait diffeilement l'importance des transformations qui ont affecté le secteur voisin.

Toutes ces transformations marquent bien pourtant l'émergence d'une ville chrétienne au scin de la cité antique et les seules inhumations suffiraient d'ailleurs a attester un profond changement de mentalite, une rupture decisive avec le vieux tabou qui rejetait les morts hors du pomerium; le plus important est sans doute cependant qu'à l'emplacement de l'ancien forum désaffecté ait été créé un nouveau pôle, éssentiel pour l'avenir de la ville : pòle religieux, mais pôle d'activité aussi dont la seule présence suffit à expliquer qu'au début du MLyen Ige le premier secteur d'Aix qui ait été marqué par la renaissance urbaine soit précisément le bourg Saint-Sauveur.

C'est l'existence de ce pôle d'activité qui nous fait croire que les bâtiments antiques du site de la Cour de l'Archevêché n'ont pas seulement été plus ou moins conservés pendant toute la fin de l'intiquité cet le haut Moyen Age mais qu'ils sont alors restés en fonction. Pour quel usage? C'est une autre question : étaient-ils encore entre. les mains de particuliers ou sont-ils entrés dès cette époque en possession de l'Église d'.lix pour constituer. à proximité du groupe cathédral, comme un quartier ecclésiastique? Faut-il imaginer dès lors ume résidence épiscopale en ces lieux? Comment savoir, quand les incessantes transformations d'un site qui ne fut sans doute jamais totalement abandonné ont fait disparaitre presque toutes les traces de l'artivité hunaine pour cette époque? Au moins semble-t-il que les modifications qui ont ensuite affecté un tissu antique conservé comme intact pendant près d'un millénaire sont déja le fait de cleres -- ou plutôt de leurs serviteurs. L’archevêque. au Moyen Ige, vit de la rente. foncière et c'est pour entreposer le produit de la collecte des grains que ses gens, des le xile sierle, ont perfore les bétons antiques.

\section{L'Éponqe: mímívale:}

On ressent un certain embarras au moment d'aborder l'histoire du site à l'époque médiévale. Cela tient à la complexité de la matière et à la difficulté de juger quel degré de détail il est nécessaire d'atteindre dans l'analyse pour justifier les conclusions. Le caractère deseriptif de la littérature archéologique est rapidement dissuasif. II risque de lasser l'attention du lecteur.

Et puis, la superficie qu’il nous a été donne de fouiller de la manière la plus intensive correspond, pour l'époque médiévale, à une partie de communs, donc de constructions fort modestes, dont l'évolution n'est suscepptible que d'éveiller un intérêt relatif. Ce n étaient, après tout, que des dépendances qui ne sauraient monopoliser l'attention vis-á-vis des constructions de prestige appartenant au même ensemble. Nais tout de même, ces mauvaises bâtisses étaient une partie de ce que l'on proposera d'identifier comme le palais archiépiscopal médiéval. Restituer l'image assez complète d'une telle construction est rarement possible. Aussi, pouvoir donner celle des communs, c'est fournir davantage qu'un contexte ou qu'un complement. C'est éprouver aussi le contraste grandissant entre la construction noble, non seulement par rapport aux habitats voisins, qu'il faut se loorner à imaginer, mais également par rapport à ses propres annexes. Ce contraste reflète bien un aspect de la vie courante à la même période. Il est atténué - ou occulte -..., à l'époque moderne comme l'illustre ici l'achèvement monumental tardif du palais par la construction de l'aile sud, sous l'impulsion de l'archevêque Charles de Vintimille, entre 1715 et 1722 environ (51). Mais cette façade, dont le recul a constitué un enjeu avant la fouille, n'était tout de même que celle des écuries (fig. 34). De technique médiocre, comme on a pu en juger, elle privilégiait l'apparence aux dépens de l'expression architecturale des fonctions. Ecran, elle a certes bien joué ce rôle, retenant l'attention jusqu'a devenir mur de scène, ce qui est un retournement spectaculaire de l'intérêt respectif des ailes du palais. Pourquoi dès lors ne pas porter une attention égale à la disposition qui l'a précédér et à des constructions qui assumaient des fonctions voisines au sein de l'ensemble monumental?

Une deuxième raison peut justifier une description attentive. C'est que l'on a eu l'occasion, par cette fouille, de constater des continuités étonnantes entre l'époque antique et l'époque médiévale. Souvent, dans ce domaine de la topographie historique, il ne peut y avoir qu'hypothèses fondées sur la méthode régressive, d'emploi délicat. Iais ici l'utilisation jusqu'à une date avancée du bâti antique en elívation, ou quelquefois des sols eux-mêmes. peut être démontrée, même si, sur les bétons des insulae, il n'y a pas eu sédimentation archéologique et itablissrment de niveaux habituellement témoins d'une occupation. Historiquement, la constatation n'est pas sans intérêt. C'est l'indice archéologique, rare pour les régions méridionales, d'une conservation du tissu urbain antique, ou au moins de certaines parties de colui-ci. I defaut de pouvoir prouver d'autres continuités, institutiomelles par exemple, cela montre que se prolonge dans la ville medievale un certain cadre materiel issu directement de l'Antiquite $: 2\rangle$.

On choisira une illustration rapide de ce phénomène, dans la partie sud-ouest de la cour. J)e l'époque la 


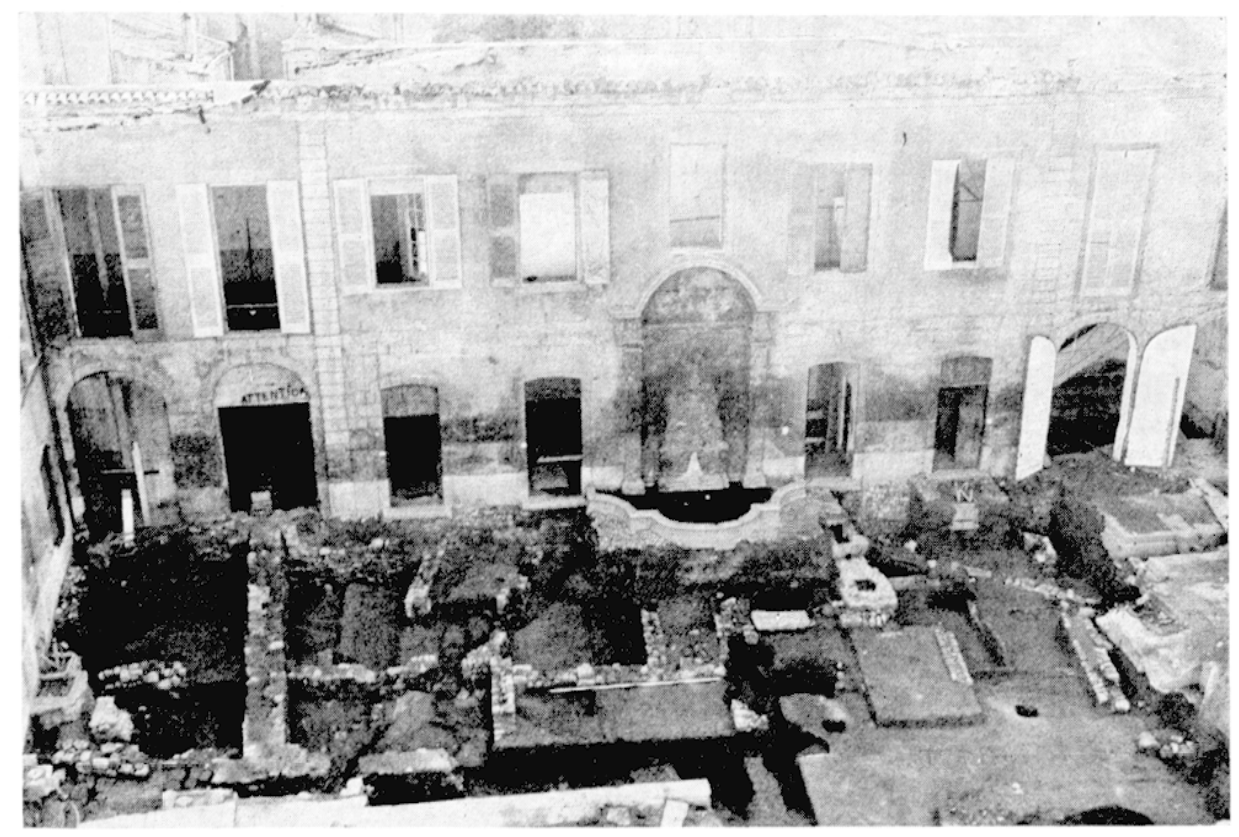

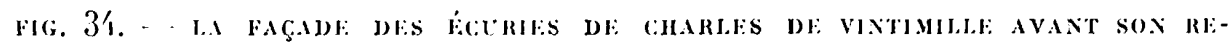

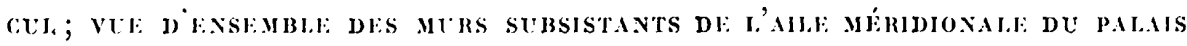
MÉDIÉVAL

plus ancienne du bâti antique jusqu'au bâti moderne, la continuité est perceptible. On se rajpelle que dans cette partie du site, le mur 11 date de l'époque de l'apparition des premières traces d'urbanisation antique. Dans une perspective évolutive, l'implantation du mur 9 semble bien dépendre de l'alignement du mur 11. Cependant, dans le plan achevé de l'insula I, l'espace $G$ est limité au sud par le mur 5 . Il y a done un décrochement des murs maîtres. I une période qu'il est bien difficile de préciser, entre le $\mathrm{v}^{\mathrm{e}}$ et le xul $\mathrm{e}$ siècle, l'angle nord-est de la pièce J2 fut coupé par le mur 56, lié a la terre, construit sur le sol en béton, et qui est apparenté par son aspect à toutes les constructions médiévales lig. 33 et 36 ). Cet aménagement introduisit une direction oblique dans le réseau orthogonal. lille fut ensuite conservée. La construction du mur 76 , dont on verra qu elle ne consiste qu'en un simple recul du mur 5 vers le sud, ne fit que l'accentuer. L'une des extrémités du mur 80 s'appuya sur celle du mur 76 , de la même manière que s'articulaient auparavant les murs 56 et 5 . De fait, le bloc des pièces de cette zone prit un plan irrégulier. Cette direction oblique dont on a suivi la génèse, commande à son tour la disposition d'un mur moderne situé un peu plus au sud, dont la fondation a été retrouvice sous le sol de la pièce occidentale des écuries

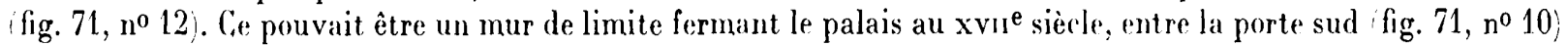
et l'entrée principale situcée déjà à l'ouest. Il fut rasé pour laisser place à la construction des nouvelles écuries et du pavillon d'entrée par Charles de Vintimille, dans une zone où l'on sait quili y eut alors agrandissementent litiges.

Continuités donc et lentes adaptations; après avoir attiré l'attention sur cette longue durée concrètement inscrite sur le site, il faut reprendre l'exposé sélon un plan chronologique.

\section{I. - UNe: probable: " DOMU'S" RoMANE}

a) Line première occupation systématique du boulevard.

Nous avons déjà dit que le Moyen Age hérite de l'Antiquité non pas de bribes éparses du bâti mais du schéma d'ensemble d'un îlot fait de pièces organisées autour d'une cour aux limites bien connues sur trois côtés sauf vers l'ouest. I.es premières modifications sensibles apportées à ce legs apparaissent dans l'espace laissé libre, ou bâti moins densément, à l'est de l'insula I. C'est cette fois une occupation systématique et pas seulement un grignotage timide de l'espace public (fig. 36).

La constatation archeologique du phenomene repose sur l'étude du nur 66 dont l'orientation est sensiblement oblique: par rapport aux lignes directrices du quadrillage antique. Il appartient à une construction relativement légère : large de $0^{\mathrm{m}} 66$, il est lié à la terre. 11 est fondé à la cote 203 sur l'argile naturelle. Cne seule assise de fondation saillante, en talon, invite à chercher le niveau d'occupation correspondant à une cote voisine de 203,20 , bien inférieure aux niveaux de circulation 


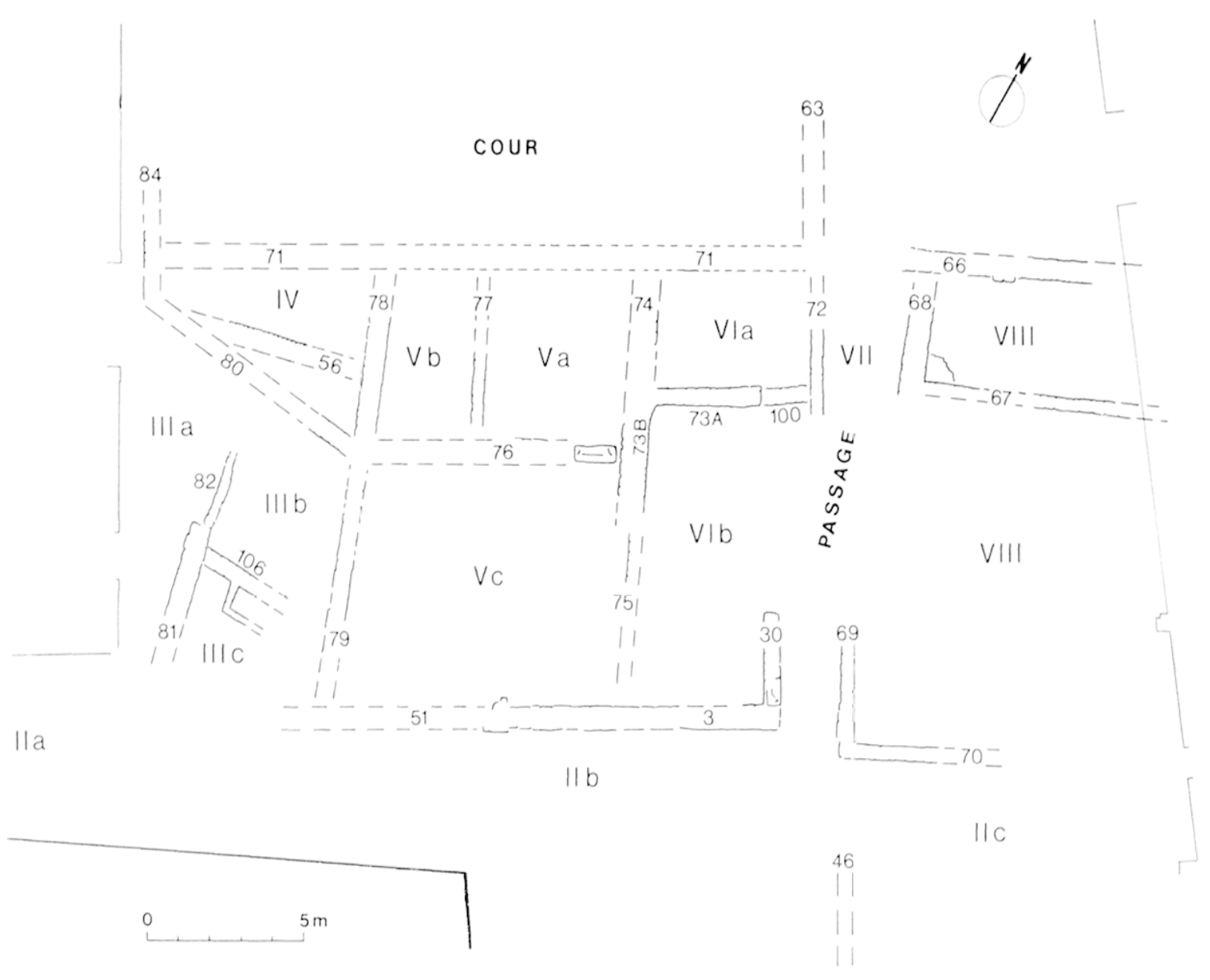

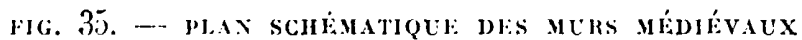

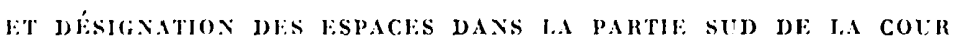

sous le portique à l'époque antique. l)e fait, vers le nord, les niveaux de remblai antique sont aussi plus élevés que le sol d'origine de ce bâtiment, dont l'organisation primitive n'est pas claire. L'un des témoignages assurés de ce volume bâti est donné, sur le parement méridional, par l'assise basse d'un support engagé, de section rectangulaire, faite de deux pierres de taille adjacentes. Elles font saillie de $0^{\mathrm{m}} 12$ sur le nu du mur. I'appareillage se fait donc dès l'assise inférieure, sans autre forme de fondation, ce qui justific aussi la recherche du sol d'occupation d'origine à un niveau has, qui est sensiblement le même que celui des bétons antiquess les plus proches, de l'autre côté du portique. Ce support engagé suggère la présence d'un arc développé vers le sud, sans doute un arc diaphragme destiné à supporter une charpente, celle d'une toiture, ou plus probablement celle d'un plancher d'étage. Cette image est importante car elle évoque une techni(̧ue de construction qui devient traditionnelle en Provence à l'époque médiévale puis à l'époque moderne dans les édifices à plusieurs niveaux (53).

On ne voit pas d'élévation susceptible de donner le symétrique du mur 66. Aussi en est-on réduit à évoquer sa disparition probable. Il ne faut pas le chercher dans le mur 67, construction extrêmement légère, simple cloison dans une adaptation ultérieure.

Mais de toute façon, le mur 67 ne délimiterait ainsi qu'un espace large de 3 inètres, distance qui rend superflue la construction d'un arc. En revanche, l'étude stratigraphique suggère bien la destruction du mur correspondant au mur 66 . Lne coupe nord-sud (fig. 37) montre que l'argile naturelle a été profondément excavée au sud de celui-ci. L.e rebord de la fosse se situe à 5 mètres au sud. Au-delà, le niveau de l'argile naturelle remonte brutalement. Aussi est-ce dans les limites de cette fosse qu'il faut chercher à restituer la largeur de l'espace primitif déterminé au nord par le mur 66, espace qui, sans être une véritable cave, avait un sol dénivelé par rapport à l'argile naturelle et aux bétons antiques. C'e niveau était susceptible de porter un étage sur plancher, étage modeste sans doute, en raison de la médiocrité des murs seulement liaisonnés à la terre et non pas au mortier. A l'est, la limite de cet espace allait au-delà de la surface fouillée. Vers l'ouest, elle n'est pas apparue bien clairement. On n'a pas retrouvé la terminaison occidentale du mur 66, car il a été raccourci ultérieurement. Aussi, la seule extrémité envisageable est-elle celle que donne le bâti antique, c'est-à-dire le mur 36 du portique qui existait encore. Ies témoignages de conservation d'un passage en cet endroit ne manquent pas en effet à une époque ultérieure. Au volume sud-est partiellement défini par le mur 66 s'ajoutaient peut-être d'autres bâtisses vers le nord. Elles restent 


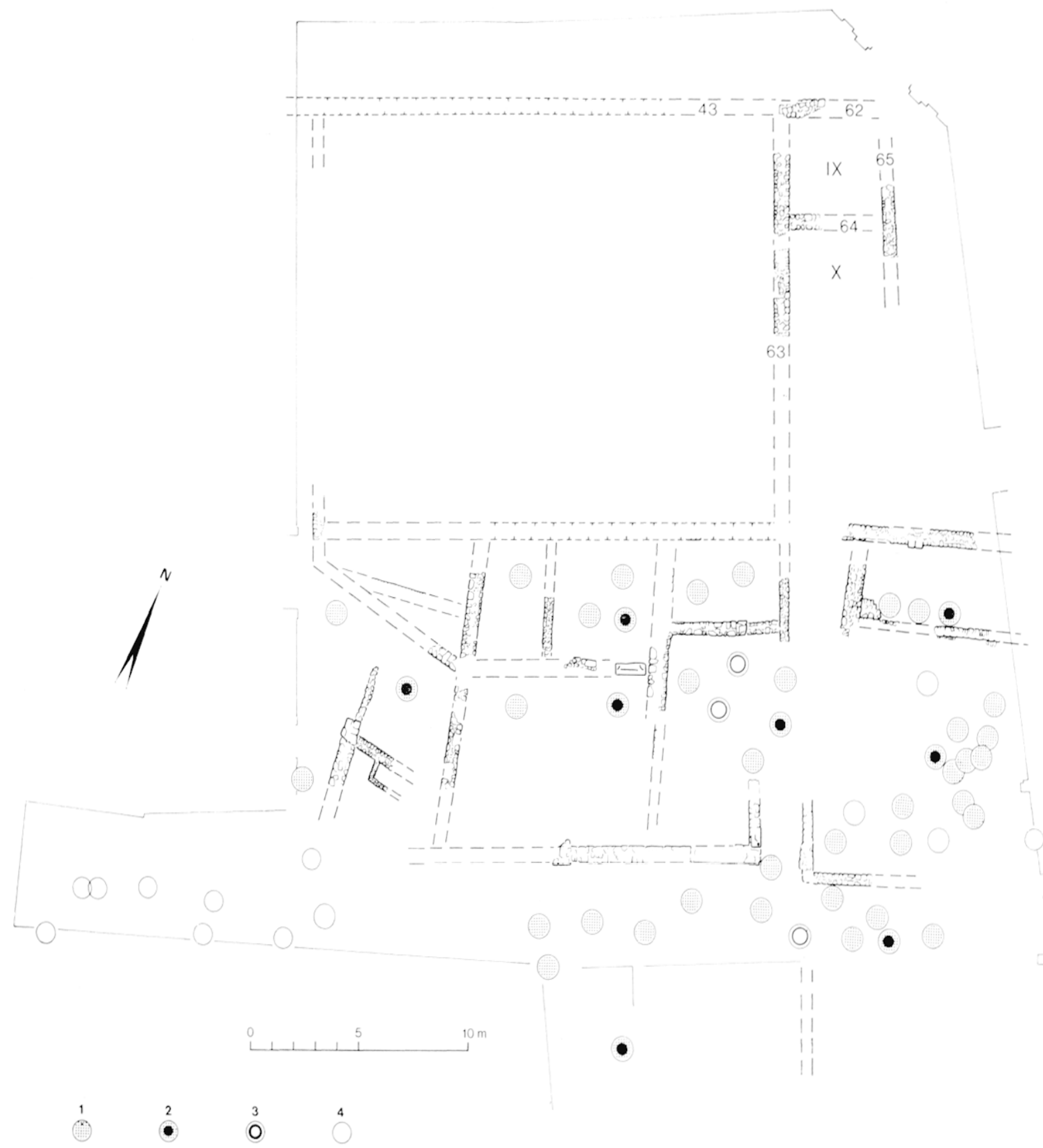

Fig. 36. - plax des constructions attribuables al palils fépiscopat de xile sièche: LES SILOS SONT DIFFÉRENCIÉS SELON LE MATÉRIEL GÉRAMIQUE Rl:CURILI DANS LEUR COMBLEMENT

1) céramique grise exclusivement ; 2) céramique grise et importations rares (vert monochrome); 3) céramique commune glaçurée associée aux types précédents ; 4) silos non fouillés.

toutefois hypothétiques. Un négatif de mur, perpendiculaire au mur 66, a été identifié dans un sondage très limité. A ce court tronçon restitué, il faut en ajouter un second, le mur 113, apparu plus au nord encore, de direction méridienne aussi, appuyé à l'extrémité orientale du mur 31 . Mais rien n'indique un rapport chronologique quelconque entre ce qui semble être un aménagement des bâtisses antiques dans cette zone, et l'apparition au sud-est, d'un projet plus précis. Le mur 66 , en effet, a été à l'origine de la disposition nouvelle de l'aile sud de l'îlot à l'époque romane. Nous verrons bientòt que de son implantation dépend celle du mur 71, élément majeur de la réorganisation médiévale. Cela prouve que le bâtiment auquel appartenait le mur 66 formait un tout avec ce qui demeurait de l'ancienne insula, qu'il en était comme une dépendance, bâtie sur le boulevard où, jusque-là, les traces d'occupation étaient inexistantes. Ce mur 66 marque aussi la naissance d'un pôle bâti dont la descendance fut très féconde. Dans l'état primitif, la présence de ce support engagé le distingue de toutes les autres cons- 


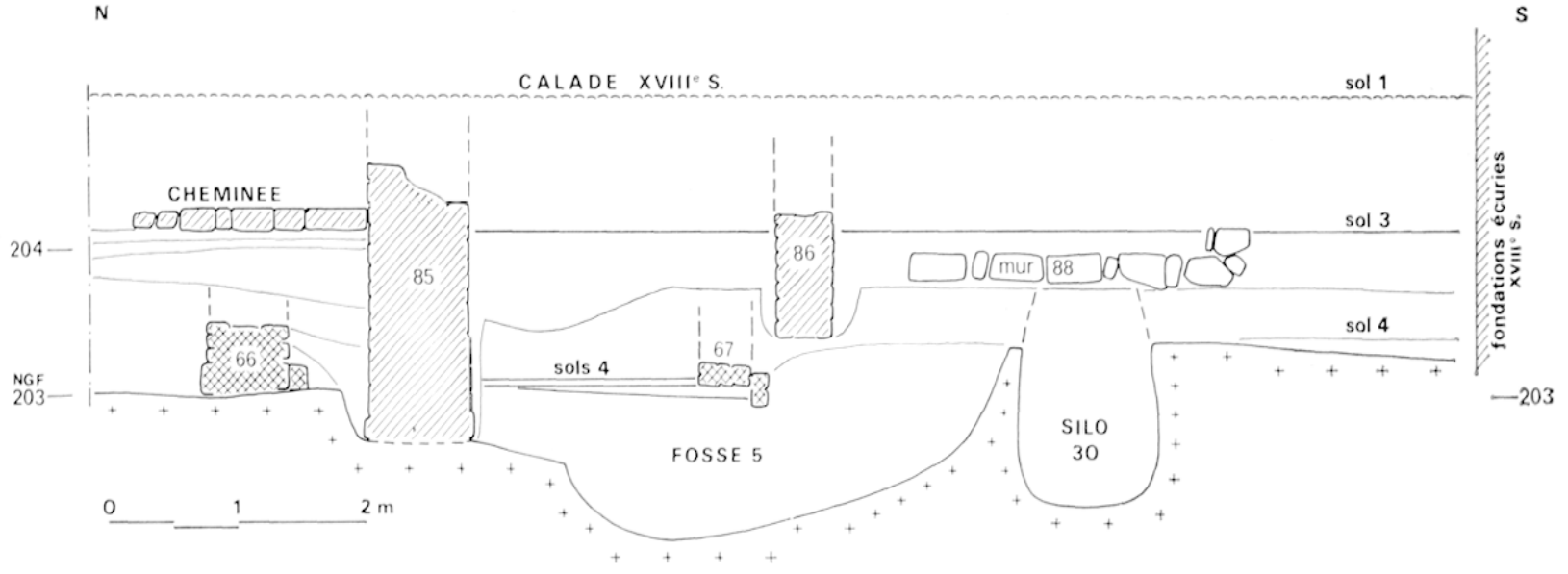

FIG. 37. - COUPE: STRATIRAPHIQUE: NORD-SUD DANS I.'FSPACE VIII ISHCTEYR SLD-RST DE LA COUR DE L'ARCHEVÉCHÉ)

tructions pour en faire un volume privilègie. $\Lambda_{u} x_{n}{ }^{e}$ siècle, nous verrons lui succéder un édifice d'une certaine qualité, dont une partie du système mural, les murs 91 et $9{ }^{\prime} \mathbf{~ e n ~ l ' o c c u r r e n c e , ~ s e r v i t ~ d ' a p p u i , ~ a u ~ x v i r i ~}{ }^{\mathrm{e}}$ siècle, à la partie orientale des nouvelles écuries de Charles de Vintimille.

En l'alsence d'indices plus précis, il faut se demander si, dans ses deux états médiévaux dont la datation sera à préciser plus bas, cette construction dont nous ne possédons plus que le mur 66, et dont nous avons essayé de marquer l'importance dans l'organisation à venir du site, n'avait pas quelque chose à voir avec l'aménagement, voire la privatisation et la garde de l'ancien portique, en l'incorporant à un îlot urbain dont le statut et la fonction étaient tels qu'il allait biontôt pouvoir être traité encore selon un projet d'urbanisme d'ensemble.

\section{b) La cour : sa limite nord.}

Les transformations qui interviennent à l'époque médiévale, pour importantes qu'elles soient, ne bouleversent pas le schéma hérité. Elles en constituent plutòt une amplification générale, suggérant que toute la partie orientale de l'insula antique fut soumise à un projet unique. C'est la raison pour laquelle nous pensons pouvoir dire que dans ce quartier proche de la cathédrale, dans une zone qui n'est pas celle dévolue aux chanoines mais qui en est adjacente, seul un édifice suffisamment important, tel que la domus de l'évèque, était susceptible d'ètre traité ainsi.

Dans son nouvel état, l'îlot s'organise toujours autour de la cour dont les linites anciennes sont repoussées dans deux directions au moins, peut-être dans trois. Ein revanche, dans la quatriène, vers l'ouest, là où dans la surface fouillie, la cour antique paraissail se poursuivre sous le bâtiment actuel, une limite est dorénavant marquée (fig. 36).

Vers le nord, la cour est alors limitée par le mur 43, ancienne façade de l'insula sur le decumanus nord, et par son prolongement, le mur $4_{4}{ }^{\circ}$. On sait qu'il est possible, mais non certain, que les bâtiments antiques des espaces $A$, appuyés au mur $\mathbf{4} 3$, avaient déjà disparu de longue date. Dans ce cas, il n'y aurait pas eu de modification à l'époque romane. Mais pour tenter une restitution du paysage bàti, il faudrait mieux savoir si ce mur d'origine antique - - ou ce qui en restait alors était simplement un mur de clôture ou s'il servait d'assise à la façade méridionale d'une construction occupant l'ancienne voie. Une telle situation n'est pas sans comparaison, au même moment, dans le quartier (fig. 32, 33 et 41). Les conditions de fouilles ont été un peu difficiles dans les zones comprises au nord du mur 43. L'état de conservation lui-même n'était pas excellent. Les pierres de ce mur et celles du mur 45 avaient été récupérées. Il n'en subsistait que les tranchées. La construction de l'aile nord actuelle est responsable de ces pertes. Mais aucun mur qui se puisse rapporter à l'état dont il est question, n'est apparu dans cetle surface, ni dans les sondages archéologiques - très morcelés -- ni lors des terrassements surveillés qui ont ensuite eu lieu.

D'autre part, il n'y a guère eu de traces de sols susceptibles d'être en rapport avec un tel édifice, sauf peut-être à l'est, dans la zone IX, au niveau 203,50 (fig. 58). On peut regretter l'absence de sondages sous le sol de l'actuelle aile septentrionale, mais le travail n'élait pas programmé dans le cadre de ce sauvetage. Restent les traces d'élévation qui subsistent dans le mur nord de la travée la plus occidentale de ce bâtiment. Antérieures à l'état gothique de celui-ci, elles présentent encore une corniche très saillante marquant le départ d'une voûte en berceau disparue, qui couvrait un espace bâti sur le tracé de l'ancien decumanus; c'est la preuve de l'occupation romane de celui-ci, au moins de manière partielle.

Ce n'est qu'en fonction de ces traces, des continuités topographiques observées sur le site, de la proximité des églises, que l'on pourrait imaginer là un grand bâtiment, sans refends inférieurs, occupant la voie antique. La restitution d'une telle aula est tout de même hypothétique.

Outre le fait d'une destruction et d'une récupération tardive des pierres, la preuve de l'utilisation médiévale du tracé des murs 43 et 45 vient de l'observation de la prolongation vers l'est des murs antiques par le mur 62 , lié à la terre comme tous les autres murs de son état. Il fut conçu pour raccorder l'ancienne élévation à la nouvelle aile orientale reportée plus à 
l'est, el pour délimiter celle-ci vers le nord. On mesurera ici l'ampleur du phénomène des continuités. La limite de la cour médiévale s'opère en effet sur l'alignement du mur 43 qui relève de l'époque des plus anciennes traces de bâti constatées sur le site.

\section{c) La façade orientale.}

I. fa f̧ade orientale sur la cour fut - radicalement cette fois - reculée de $12^{\mathrm{m}} 50$. Cette nouvelle façade, identifiée avec le mur 63, occupait l'ancien portique dont elle suivait l'axe. Que restail-il alors des élévations dans les pièces $\mathrm{B}, \mathrm{E}, \mathrm{F}$, $\mathrm{N}$ et M? On doit se résoudre à l'ignorer dans la mesure où, dans cette aile, les constructions médiévales n'ont tenu aucun compte des préexistences. Le matériel céramique retrouvé sur les bétons antiques ne dit pas si l'on se trouve alors à l'intérieur ou à l'extérieur de bâtiments Le mur 63 était large de $0 \mathrm{~m} 80$ pour sa seule assise conservée, mais l'élévation pouvait être plus étroite. Il a été suivi, vers le nord, sur une longueur de $9^{\mathrm{m}} 50$, en deux tronçons. Il a été retrouvé ensuite au contact de la tranchée du mur 45 , lié au mur 62. L'articulation montre la simultanéité de construction des murs 62 et 63 . Elle confirme l'appartenance du mur 62 au système médiéval. De ce mur ne subsistait également qu'une assise, exceptionnellement deux. A 1 m50 à l'est de sa jonction avec le mur 63, il était interrompu par un élément, sans doute un jambage de porte. Sa disparition totale, plus à l'est, est due à la perturbation apportée par le creusement ultérieur d'un atelier de fonte de cloche. Selon les deux restitutions possibles exposées ci-dessus, cette porte aurait donné, soit sur un espace ouvert, soit dans une grande salle de l'aile nord.

Faibles sont les traces retrouvées qui peuvent donner une idée un peu précise de ce qu'était l'aile orientale. Les murs ne sont apparus qu'en fondation et aucun sol correspondant n'a été repéré. Les conditions étant semblables ainsi à celles rencontrées au nord, cela confirme qu'il ne faut pas finalement s'empresser de conclure à l'absence de construction sur l'ancien decumanus. Mais contrairement à ce qui pouvait se produire dans l'aile nord, l'espace intérieur de l'aile oricntale était cloisonné. Il demeure de cela les murs 64 et 65 , liés entre eux et dont les conditions de conservation ne sont pas meilleures que celles des murs de toute la zone. C'est dire qu'il n'en demeurait aussi qu'une ou deux assises parfois, a vec liaison à la terre.

Plus au sud, une assez vaste zone a échappé à la fouille (fig. 2).

\section{d) L'aile sud.}

Les renseignements sont plus précis pour l'aile sud. Il n'y a pas lieu, selon nous, de suspecter que la différence des résultats tienne beaucoup au rythme de fouille, moins précipité ici que là. La différence est due surtout à une meilleure conservation des vestiges et des sols dans une partie du site moins noble sans doute, mais aussi moins sujette aux reconstructions radicales, et dont les élévations, plus longtemps conservées, n'ont subi que des évolutions lentes (fig. 35 et 36 ).

Ia façade nouvelle de l'aile sud coupe au travers de l'insula I. Mais, comme celle de l'aile orientale, elle respecte scrupuleusement le parallélisme par rapport aux axes antiques. Cette façade, c'est le mur 71, essentiellement révélé par la tranchée de fondation, et de récupération, des matériaux. l)ans sa partie médiane, quelques pierres du parement sud ont échappé à la pioche des récupérateurs et confirment la restitution. T'rois pierres aussi, à l'est, au contact du mur 66 précisent l'alignement. Une restitution certaine est donc possible, d'est en ouest, sur une longueur de 17 mètres. Au-delà, dans une zone fouillée plus vite, la trace n'a plus été reconnue. Par rapport à la situation antique, le recul de façade est de l'ordre de $2^{\mathrm{m}} 50$. Il tient compte des élévations existantes. De la plus récente d'abord et, en l'occurrence, du mur 66 sur l'extrémité arrachée duquel le mur 71 vient prendre appui. Il faut maintenant s'arrêter un moment pour justifier ce qui a pu apparaître jusqu'ici comme un postulat, qui est l'antériorité du mur 71, et du bâtiment qui en dépendait, par rapport au remodelage de la cour que l'on est en train de décrire. On objecterait à bon droit que les trois pierres conservées de l'extrémité orientale du mur 71 pourraient appartenir à l'obturation plus tardive d'un espace laissé ouvert à l'origine entre les murs 66 et 71 . Ainsi le rapport d'antériorité du premier sur le second ne pourrait être démontré. Ce serait oublier que la démolition partielle du mur 66 s'inscrit trop dans un projet logique d'aménagement de l'espace qui a pour but le dessin d'une nouvelle cour, tout en conservant un accès vers l'intéricur de l'îlot. La construction du mur 68, nouveau mur de passage, au droit de la coupure du mur 66 , répond à un tel objectif. La nouvelle articulation est soulignée par la mise en place d'une chaîne d'angle faite de deux blocs antiques de remploi. Le résultat permet la conservation d'une circulation, celle qu'offrait déjà l'ancien portique le long de la façade orientale de l'insula donnant sur le " boulevard ", mais en la détournant. L'espace VIII, remodelé mais toujours limité au nord par le mur 66 raccourci vers l'ouest, gardait le rôle qui avait été le sien avant l'agrandissement de la cour. Il flanquait toujours l'entrée - ou un accès - vers l'intérieur de l'îlot. Il conservait le souvenir du système de circulation antique, mais avait èté légèrement déplacé par rapport à lui.

Il n'était peut-être pas inutile de s'arrêter sur ce point ; il peut paraître de détail, mais il a valeur d'exemple. Il montre encore l'ampleur des continuités. Pour qu'un tel remodelage de l'espace ait cu lieu, il fallait que la circulation sous l'ancien portique, privatisé sans doute déjà, existât encore au moment des transformations décrites, et que la nécessité de sa conservation fût ressentie. L'exemple montre aussi comment se modifie, par glissement, la voirie ancienne d'importance secondaire, pour former la voirie médiévale. L'exemple n'est pas exceptionnel dans l'archéologie du haut lloyen Age, il l'est peut-être pour les villes antiques de Provence, où de telles continuités n'avaient été signalées que pour des axes majeurs.

Lors du recul de la façade méridionale de la cour antique au moment de la construction du mur 71, des murs existant encore sur les fondations anciennes de l'insula ont été abattus. Semblable phénomène de conservation des volumes d'origine antique jusqu'à l'époque de l'agrandissement systématique de la cour vient d'être montré pour le portique. On peut le montrer maintenant pour la partie médiane de l'aile sud. En dépit de la nouveauté du projet, le bouleversement fut loin d'être total. In arrière de la nouvelle façade, l'image antique subsista longtemps encore avec force.

I.'héritage était constitué par un corps de bâtiment de forme trapue. Depuis qu'au sud, l'espace de circulation avait 


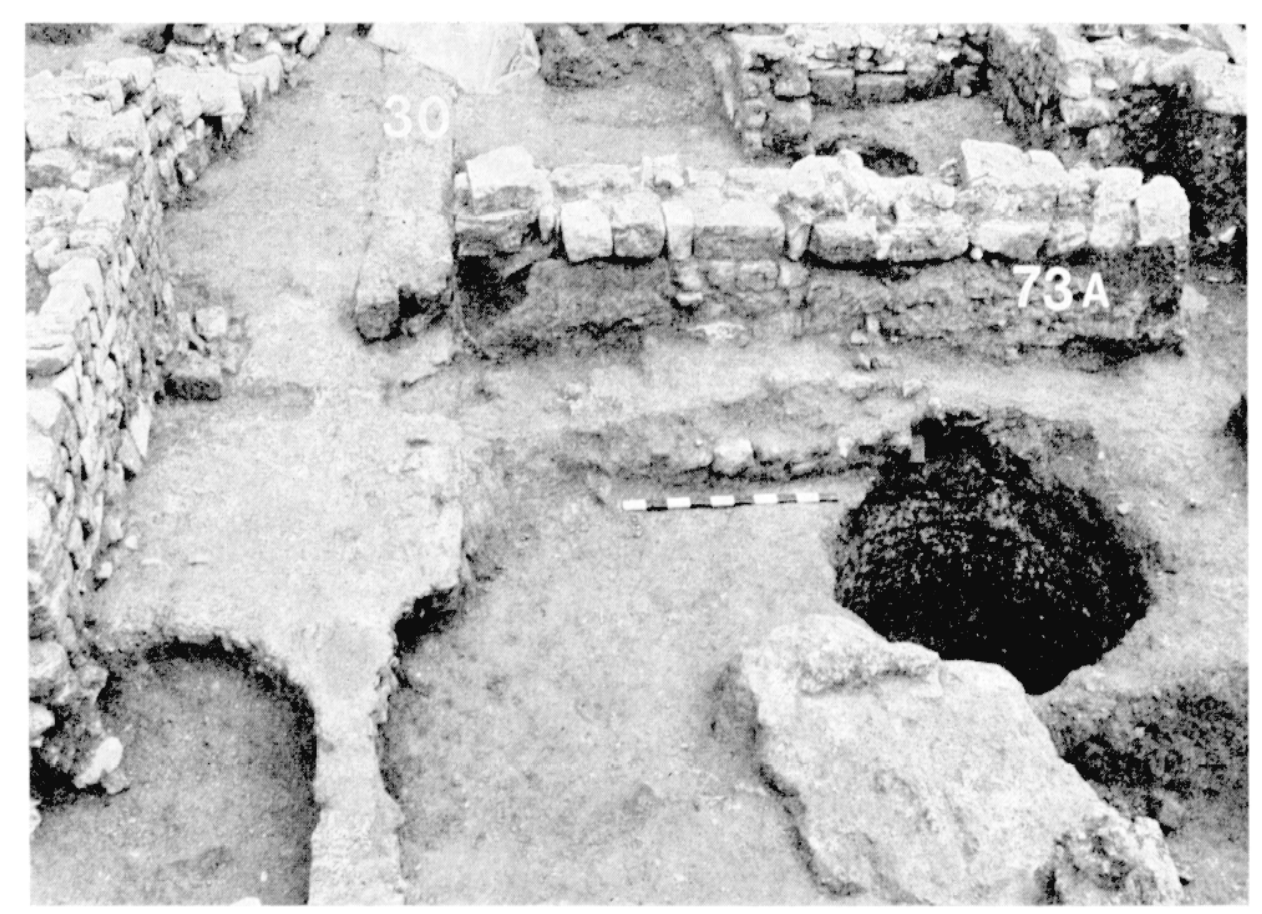

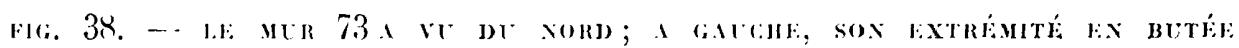

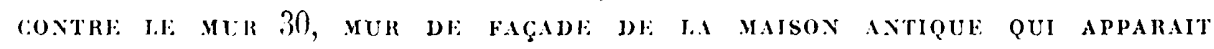

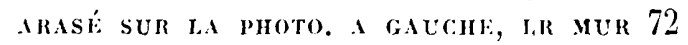

été occupé, la construction comprenait en profondeur trois rangées de pièces. Le mur antique 51 demeurant mur de fond, le seuil de la nouvelle façade réduisit l'organisation des volumes à deux rangées seulement. Mais dans l'espace V, de manière étonnante, on chercha à conserver aux pièces donnant sur la cour la largeur de celles qu'elles remplaçaient.

Aussi leur mur sud, sous la forme du mur 76 , retrouvé en négatif seulement, fut-il bâti en retrait du mur 5 , à une distance identique au retrait du mur 71 par rapport aux murs 15 et 17 . La largeur des pièces $\mathrm{V}$ a et $\mathrm{V}$ b était du coup semblable, à $0 \mathrm{~m} 50$ près, à celle des espaces $J 3$ et $J^{\prime}$ auxquels elles se substituaient. Vieux même, le respect des volumes allait de pair avec la reproduction du dispositif de circulation. Ainsi, entre V c et V a on plaça un seuil, un remploi vraisemblablement, dont l'emplacement se trouvait dans le même axe que le seuil piéton qui, dans le système antique, séparait I)3 et J4. Cela pourrait d'ailleurs suggérer qu'entre-temps, la porte adjacente, plus large, avait pu être condamure entre des espaces devenus tous deux intérieurs et que l'accès le plus étroit suffisait à desservir dorénavant.

Vers l'ouest, la conservation des lignes fut également remarquable. Il en a été question plus haut déjà, à titre d'exemple. I a nouvelle implantation des murs 71 et 76 eut, on le sait, des conséquences sur l'agencement des espaces IV. On a vu comment l'obliquité du mur qui coupait l'angle nord-est de l'espace J2 se trouva accentuée par la construction du mur 80 , dont l'implantation dépendait maintenant - et logiquement --- du mur 76.

L'organisation intérieure de l'espace médiéval héritait donc ici aussi du monument antique progressivement remanié. Jans cette partie occidentale de l'aile sud, cependant, cetle accentuation entraina l'adoption d'un cloisonnement qui est apparemment nettement en disharmonie avec les lignes orthogonales antiques. Ces cloisonnements s'organisìrent néanmoins en prenant comme appui un pilier grossièrement quadrangulaire bâti dans l'arrachement du mur 1. Cette implantation montre que même dans cette partie, la plus étrangère au schéma antique, il y a des réminiscences dont on ne comprend d'ailleurs pas très bien la raison, si ce n'est, peut-être, la conservation de certaines lignes de charpente pour l'organisation des toitures.

L'organisation des pièces, en profondeur, conserve donc suffisamment la mémoire de la disposition antique pour que l'on puisse conclure à la subsistance des agencements majeur's jusqu'à l'époque de l'agrandissement de la cour. On peut même dire que l'on chercha à la prolonger en dépit des transformations. Ia conclusion est aussi claire, plus encore s'il est possible, quand on étudie les nouveaux cloisonnements nord-sud en rapport avec ceux des volumes anciens. Les limites entre les pièces paraissent purement et simplement remplacées ou transposées. $\Lambda$ la technique de construction au mortier et à l'enduit peint sur les parements se substitue simplement une maçonneric qui nous paraitt plus fruste, utilisant des mœllons antiques rícupérés, liaisonnés à la terre.

Commenģons par l'ouest. I. ('s murs 78 et 79 se substiluent respectivement au mur 1't et à la cloison 24. Ieur tracé, un peu oblique, cherche peut-être à s'harmoniser avec l'accentuation de la déviation antique qu'opère simultanément les mur 80 pour des raisons maintenaut bien connues. Mais le respect de la disposition antique se lit jusque dans l'épaisseur respective des murs. De part et d'autre de l'appui du mur 76 , comme c'ćtait le cas naquère de part et d'autre de l'appui du 


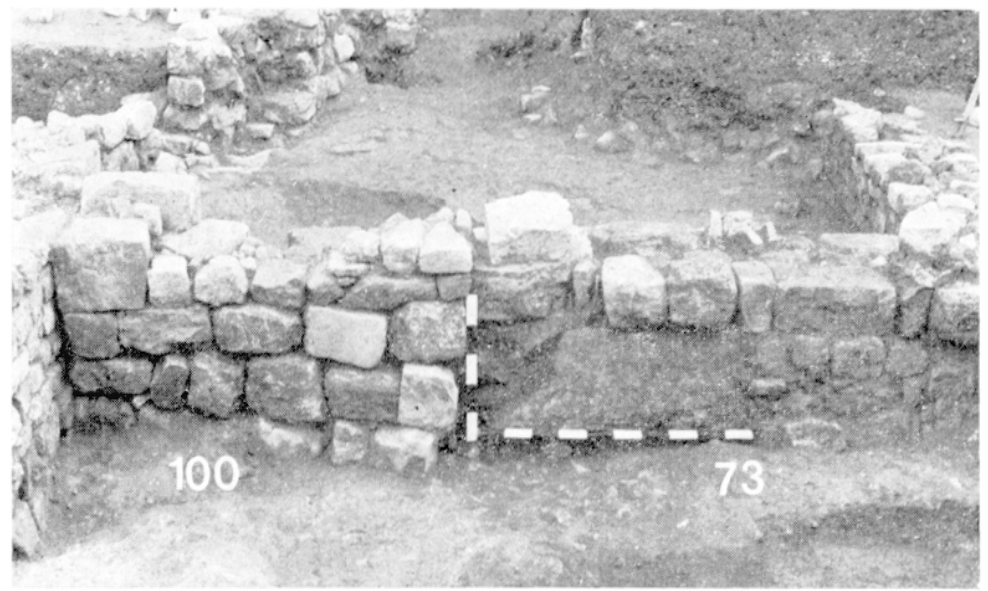

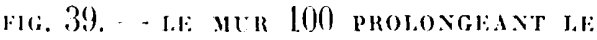

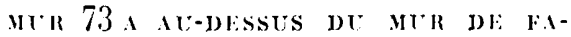

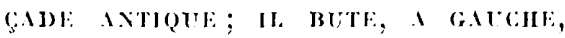

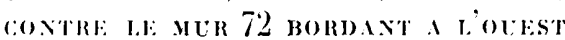

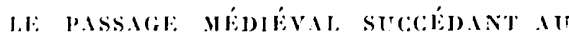
PORTIQT:

mur 5, le mur nord, le mur 78 , est un mur maître, et le mur 79, au sud, n'est qu'une cloison. L'aile médiévale apparaît de plus en plus comme un dicalque de la maison antique. 1)e mème, le mur 77 est une cloison entre les espaces $\mathrm{V}$ a et $\mathrm{V}$ b. Ce n'est que li reprise du mur antique 22, cloison entre les espaces J3 et J/4, transposée au nord du mur 76. Un démarquage semblable par rapport aux données antiques a été déja noté pour le seuil interrompant le mur 76 .

Plus étonnantes encore sont les observations faites dans l'espace VI qui nous ramènent à la topographie du passage, simple démarquage du portique. Il est probable qu'ici, comme dans l'espace VIII pour le mur 66, ou qu'en IV a pour le mur 56, certaines transformations ont précédé la nouvelle délimitation de la cour. Mais nous verrons alors que leur datation nous échappe. Ce que l'on a pu lire daus l'espace VI, c'est que le mur 7, d'origine antique, fut doublé au sud par le mur $73 \mathrm{~A}$. Semblable construction conduisait à reproduire, dans cette partie de l'insula, le système des parois adjacentes existant dans cette zone, en particulier pour les murs 2 et 28 . Vers l'est, le mur 73 A vint buter sur le mur 30 , mur de façade de l'insula (fig. 38). Je cette limite ancienue, le mur $73 . \mathrm{A}$, repris dans les constructions médiévales, a gardé sa terminaison franche que l'on ne doit pas confondre avec un jambage de porte. Vers l'ouest, ce mur 73 faisait angle vers le sud : il venait s'appuyer en biseau contre le mur 4, mur de la génération antique la plus ancienne. l.orsque les pierres des murs 2 et 4 furent récupérées a l'époque midirvale, on ne touchat pas au mur $73 \mathrm{~B}$ dans son retour meridien puisqu'il délimitait l'espace VI b. Aussi a-t-on parfaitement observé, au cours de la fonille, comment la queue des moellons de ce mur venait s'aligner sur le rebord du béton de la pièce $K$, c'est-i-dire contre le parement oriental du mur $\mathcal{A}_{1}$ disparu. Line telle adaptation du plan antique régularisait le tracé un peu oblique du mur 's et conchait plus harmonieusement, vers le sud avee un biseau, l'épaisseur double des murs 2 et 28. Sans avoir élé réalisí au mortier, la liaison à l'argile jaune des pierres du mur 73 individualisait bien la campagne de construction.

Que se passa-t-il au moment de l'agrandissement de la cour? Ici encore les transformations opérées démontrent que le bati d'origine antique subsistait. I.e mur 71, au nord, trancha l'ancien espace pour s'adapter à l'alignement du mur 66. On ne repoussa pas vers le sud la cloison du pelit volume qui résultait comme on l'avait fait pour le mur 76 , dans l'espace V. Cela indique sans doute une hierarehisation des espaces, la pièce VI a étant d'importance secondaire par rapport à la pièce V c. On ouvrit simplement entre les pièces $\mathrm{V}$ a et $\mathrm{VI}$ a en finissant de détruire les murs doubles 2 et 28 qui avaient déjà dû être rasís sur presque toute la longueur lors de l'agrandissement de la cour vers l'est. Puis, pour donner tout de même un certain volume en VI a, on détruisit le mur 7 . Seul alors le mur qui l'avait doublé au sud, le mur $73 \mathrm{~A}$, subsista. La fouille permit de constater un curieux relournement de situation. L'enduit de tuileau qui avait protégé lo parement sud du mur 7 était conservé contre le mur $73 \mathrm{~A}$ avec une élivation d'une cinquantaine de centimètres, preuve s'il en est que les deux murs avaient existé simulaníment pendant un certain temps. lors de la récupération des matériaux du mur 7 , son enduit n'avait pas éte détruil.

Il fallait achever de délimiter l'espate VI a ; lo mur 72 fut élevé dans le prolongement du nur 63 , nur de la nouvelle façade orientale. I a diférence d'épaisseur entre les murs 6.3 et 72 montre qu'il y avait entre eux une interruption majeure que constitue l'angle sud-est de la nouvelle cour a la jonction entre les murs 71 et 63. Le mur $73 \mathrm{~A}$, arrêté naguere contre le mur 30, fut prolonge par le mur 100 jusqu'au mur 72 qui limitait le passage et consacrat, comme on l'a expliqué, le glissement, ou la déviation de celui-ci vers l'est (fig. 39).

T'erminons rapidement avec l'espace VI b. Ie mur 73 formait l'angle nord-ouest de la pièce. On reprit par le mur 75 l'ancien alignement du mur 4 . Ainsi se superposerent un mur du système antique le plus ancien et le mur médiéval, fait encore de mollons de remploi liaisonnis a la terre. Il est probable que de cette époque date la destruction du mur 3 , dans le but de donner une surface suffisante à l'rspace VI b. C'est a ce moment aussi que l'on peut placer l'installation d'un seuil

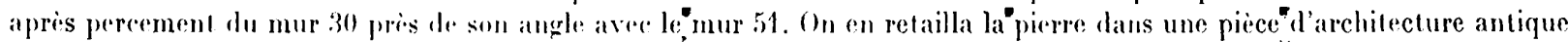

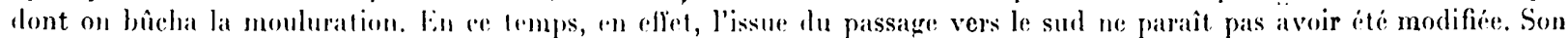
débouché sur le decumamus sernble toujours s'eflectuer ant le les murs 30 et 36 , avec un trace oblique que nous verrons mieux se matérialiser dans l'évolution postipreure. Kn eflet, pour les premiers temps qui suivirent le nouveau dessin de la cour, une incertitude demeure sur le tracé médian du passage et sur l'organisation des espaces adjacents, en particulier vers l'est. 
Rien ne vient structurer le volume compris entre le mur 67, qui n'est pas un mur maître, mais une cloison, et le mur 70 .

La construction de celui-ci témoigne d'une disparition des lignes du bati antique dans cette partie du site. A l'ouest, c'était toujours le mur 51 , d'origine antique qui faisait façade sur l'ancien decumanus. $A$ l'est, en revanche, la reconstruction du mur 70, qui remplą̧ait le mur 49 détruit, avait empiéte sur l'ancienue voirie.

\section{d) Les niveaux d'occupation et les silos.}

A l'emplacement d'une éventuelle aile nord et de l'aile est, la fonille n'a pas donné l'occasion de retrouver des niveaux d'utilisation correspondant aux murs découverts. On a d'ailleurs pu constater que ces murs sont apparus en fondation et non en élévation. Les chantiers médiévaux ultérieurs ont cntrainé, dans toutes ces zones, des terrassements importants aux méfaits desquels s'est ajouté le crensement de nombreuses fosses destinées soit a la fabrication de la chaux, soil a la fonte de cloches. In sol médieval est apparu au nord-est, entre les cotes 203,50 et 203,60, dans la surface circonscrite par la tour noderne. Elle est trop étroite, on l'a dit, pour permettre unc caractérisation quelconque. Et puis, le dégagement rapide des terres au nord de la cour actuelle juscqu'au niveau antique, la conservation imposée d'un talus de terre au pied de la laçade nord pour d'évidentes raisons de stabilité ont gêné les observations. Les explorations ponctuelles dans cette zone n'ont pas donné dans cette recherche des résultats bien nets. Ce ne fut pas la surveillance ultéricure des terrassements qui était susceptible de faire découvrir des sols de terre battue.

Dans l'aile sud, en revanche les donnees furent plus précises. Les sols d'occupation médiévaux lies au remodelage de la cour présentent des situations diverses. Dans la zone centrale, dans les espaces Vet VI a, il y a eu remblaicment sur les bétons antiques. Le remblai ful contenu - coflré - entre les murs 71 et 51 , au nord et au sud, et entre les murs '4, 73 et 72 à l'est,

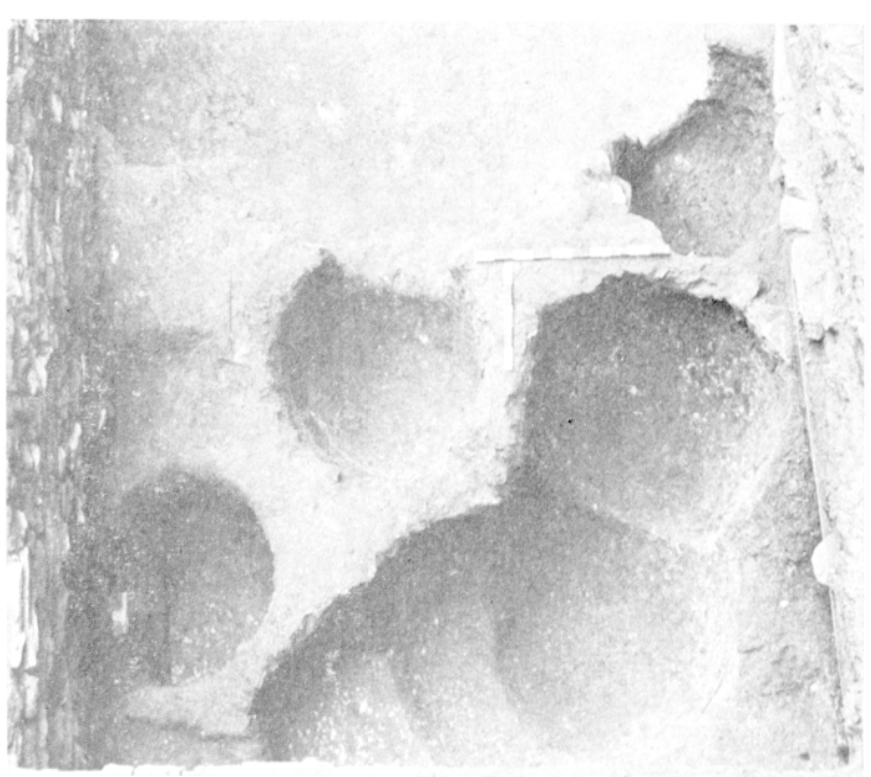

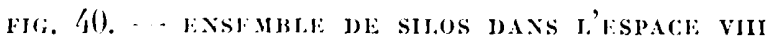
et 79 enfin a l'ouest. Dans le cas des murs 75 et 78 , ce sont, pour ainsi dire les parements extérieurs à la surface remblayée qui ont contenu le comblement, c'est-à-dire le parement vuest pour le mur 78, ou la préexistence du mur 73 pour le mur 75 . l'uis dans les deux cas, les parements intérieurs ont été posés sur le comblement réalisé. Cela a entraîné une disșmétrie de ces murs en coupe, les parements intérieurs étant plus élevés que les parements extérieurs qui se trouvaient posés sur les bétons antiques.

Cette observation confirme la liaison qu'il faut établir entre le comblement et les consiruclions liécs au remodelage de

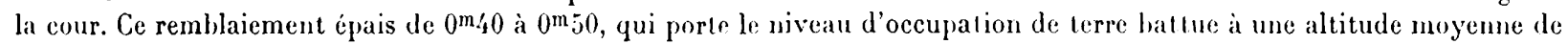
$204 \mathrm{~m}$., s'est effectue en une seule fois, par tes juxtaposiss et nivelés. Le matériau est une argile assez plastique, plus ou moins sombre selon les tas, contenant des débris de dénolition fins, nodules de mortier ou fragments d'enduits. Il y a en tout cas absence de toute pierre susceptible d'ètre réutiliséc dans la construction. Aussi, après le comblement réalisé en une seule fois, l'espace ainsi determiné fut-il recloisonné par la construction des murs $76 \mathrm{et} 77$, et l'on recopia, comme on l'a vu, les volumes détruits. Tout cela permet d'aflirmer que de mème que les elévations antiques, comple tenu de modifications possibles dans les parties hautes, ćtajent utilisées jusqu'à l'époque du remodelage de la cour, de mème, les sols antiques étaient encore en usage et régulièrement nettoyés jusqua la mème période. Wans les espaces III et IV, ou le mur 80 et la cloison 82 sont posés sur les sols de beton, cette utilisation s'est prolongée encore, noins longtemps cependant que dans l'espace VI b.

L'espace V fut donc un temps surelevé par rapport aux espaces latíraux. Son sol d'occupation fait de pellicules ou de lentilles de sol ceudreux formant terre battue passait exactement au-dessus des anciens seuils et des arases les plus élevées du mur 3 qui venait d'ètre détruit.

Autre situation, celle du passage. Du portique ancien, le long du boulevard, le passage VII n'a pas conservé seulement le trace. Il en a aussi repris et accentué la dénivellation par rapport aux espaces arljacents VI b et VIII. $\Lambda$ l'est, de son origine antérieure au dessin de la nouvelle cour, l'espace VIII conservait en effel un sol bas. Apres diffírentes transformations, creusement puis obturation d'une grande fosse énigmatique, et construction d'une cloison 67, l'établissement du nouveau sol n'était que le reflet de celui d'origine, encaissé dans l'argile naturelle. (1n y a fouillé des niveaux feuilletís ou stratifiés de terre battue en accumulation horizontale regulière (fig. 37 ).

Il faut enfin dire que l'aile sud et ses abords meridionaux, par rapport aux aul res espaces ouverts ou fermes, se sont parfaitement individualisés grâce à certains aménagements. Cela a confirmé l'importance de la limite que forme l'alignement des murs 66 et 71 dans l'ensemble du bâti. le mur 71 n'ayant èté, après tout, présenté que comme restitution fondée sur l'observation d'un négatif, un argument supplémentaire n'est pas inutile pour s'assurer definitivement de son existence. Ces aménagements sont des silos.

On sait que l'on ne fouille guicre de site d'habitat médieval, il la campagne comme a la ville, sans rencontrer ces cavi- 


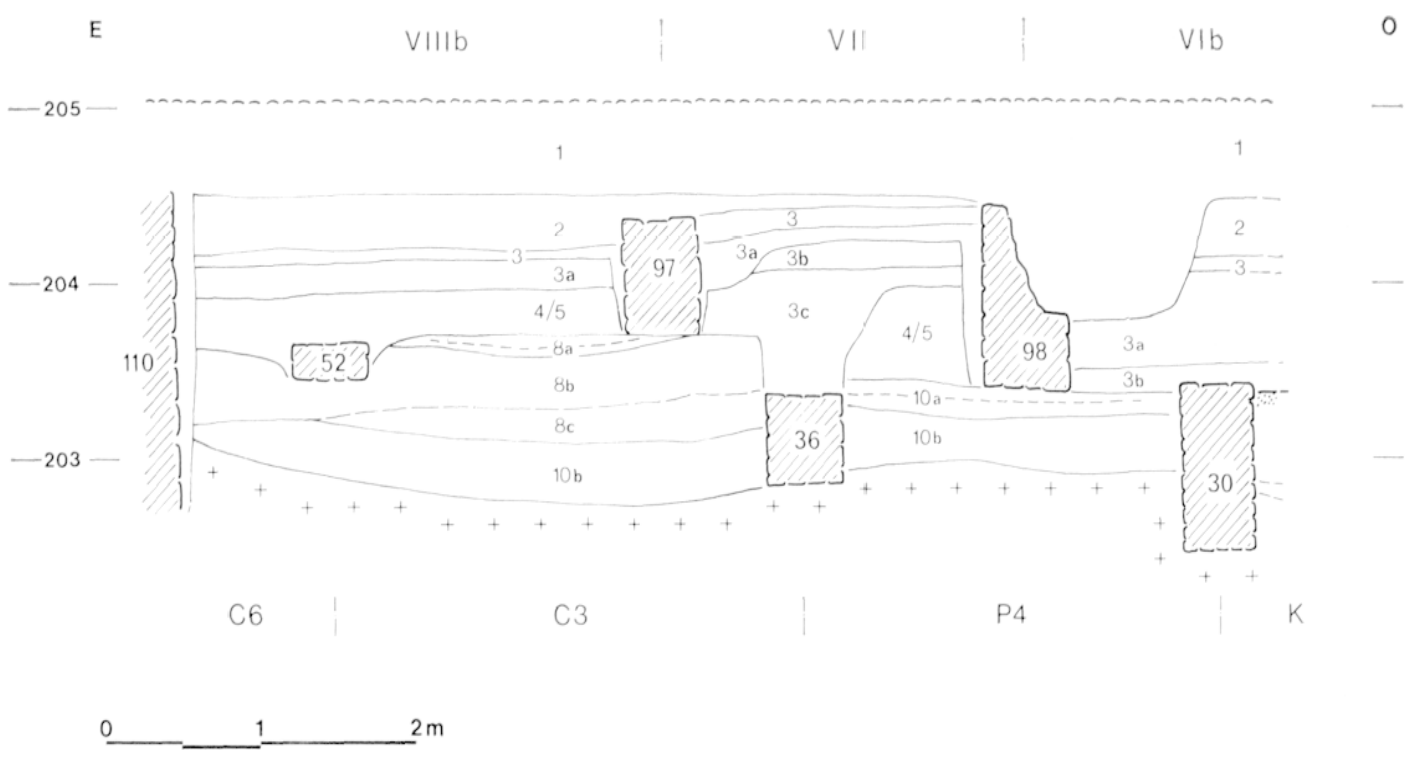

FIG. $41 \mathrm{~A}$

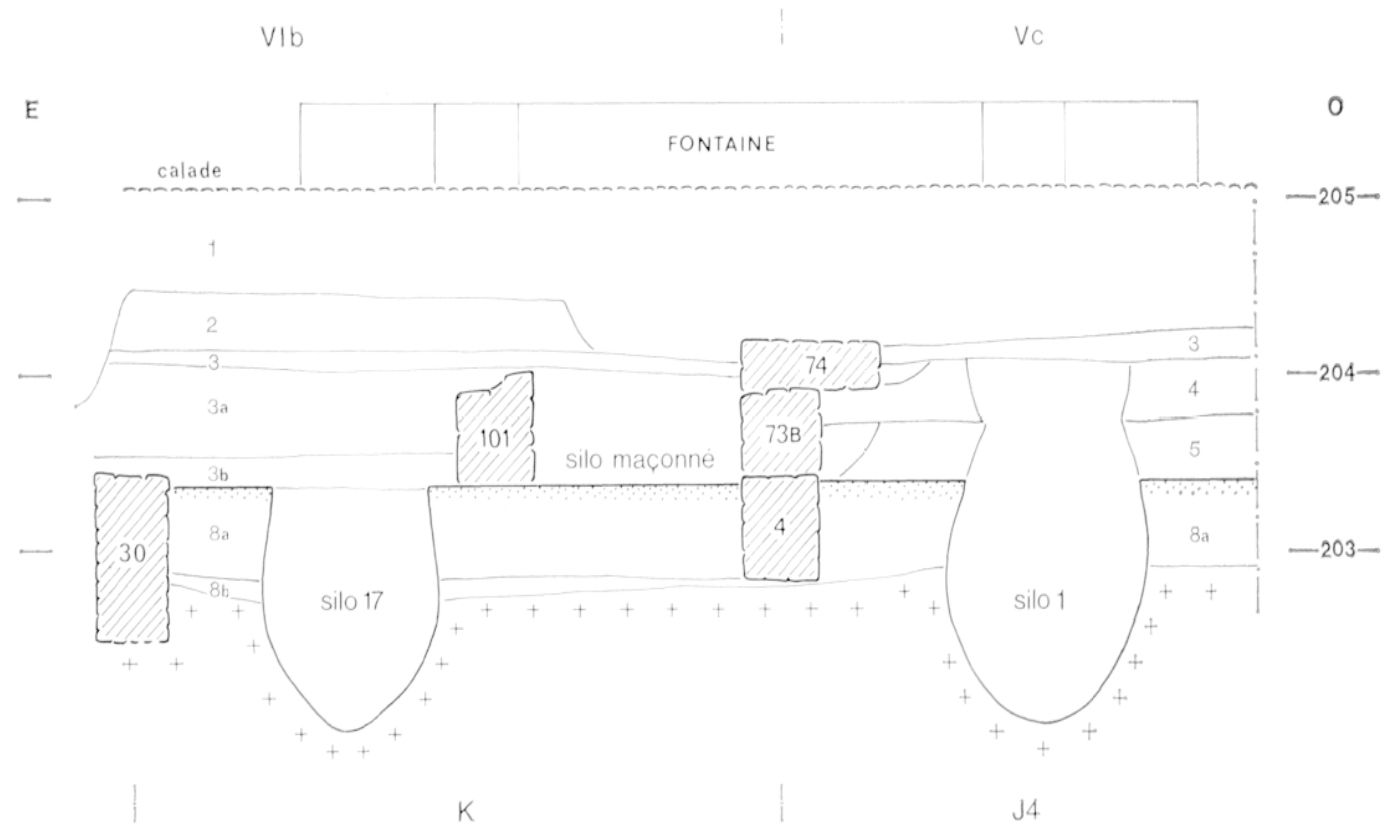

FIG. 41 B

FIG. 41 A FT B. - COUPE STRATIGRAPHIQUE OUEST-EST

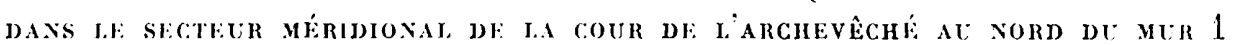

1. Antiquité : 1) Dans les espaces du boulevard (?), entre les murs 110 et 36 , et du portique, entre lcs murs 36 et 4) : couche 10b : remblai brun orangé sur le sol naturel dont le profil montre des entailles; couche 10a : fins remblais supportantun sol chaulé; couche $8 \mathrm{c}:$ fosse comblée de nombreux fragments d'amphores et de dolium; couche $8 \mathrm{~b}:$ remblai sa blonneux ; couche $8 \mathrm{a}$ : niveau de circulation en terre battue, avec recharges $\left(2^{\mathrm{e}}\right.$ moitié du ${ }_{11}{ }^{\mathrm{e}}$ siècle). 2) Dans l'espace $\mathrm{K}$ : couche $8 \mathrm{~b}$ : couche cendreuse, de couleur noire: sur le sol naturel; couche 8 a : remblai sur lequel est aménagé le sol en béton de tuileau. II. Hoven Age : couche 5 : niveaux de remblaiement et de récupération préédant la construction du palais roman; couches 4 : couches portant les sols d'occupation romane; couche $3:$ sol d'argile du $\mathrm{xIV}^{\mathrm{e}}$ siecle (dernière occupation de l'aile sud); couches $3 \mathrm{abc}$ : sols d'occupation et remblaiement dans le courant du xiv siècle; couches 2 : destruction de l'aile sud. III. Époque moderne : couche 1 : remblaiements, tranchées diverses et fondations de la fontaine sous la calade. 
tes de forme régulière, creusées dans le sol, et destinees a la conservation des récoltes, grains ou pois, selon des methodes

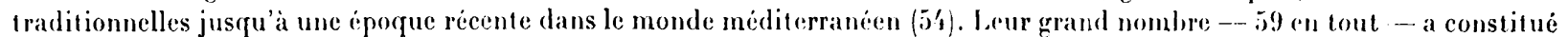
une " rallonge" d'environ 100 metres cubes à la fouille des niveaux medievaux. (1n sait que ces cavitis servent fréquemment de drepotoir quand cesse leur utilisation. Hais, en dépit de cette richesse potentielle, la fouillr a dù en être accilérée en raison des conditions de rapidite exigées.

La ripartition de ces réserves est reguliere dans la plupart des espaces de l'aile sud (fig. 36). Il s'en trouve dans chacun, sauf en IV a. En III c, il en existe une, de type un peu different des autres. Ses parois sont construites en pierres seches, et elle est de plan quadrangulaire. lin général, la repartition exclut tout recoupement, a l'exerption de ce qui se passo dans l'ensemble très dense situé à l'est, en VIll, qui fait contraste d'ailleurs avec la region vide adjacente (fig. 10).

En general la partie superieure de ces silos est crensier dans les niveaux archeologiques. Puis les betons antiques ont ili perforis, sans difficulté, ainsi que leur radier, et la caviti s'ipanouit dans l'areile naturelle compacte (fig. 41). Parfois en maison de la faiblesse diune paroi, celle-ci fut renforce localement, ou sur toute la circonference, par un bati de pierres siches (fig. 42 l. Cela est asse\% frequent pres des embonchures creuses dans des sols de remblais plus meubles que largile naturelle. Les profils sont toujours réguliers, soil ovoüdes, soit piriformes, avec dans ce cas un fond plat (fig. '33! Les parois sont loujours tres lisses. Les contenances sont de lordre de 1 a 2 melres cubes. Lin raison de leur affleurement dans les niveaux archiologigues, les embenchures ne furent pas toujours immediatement pereeptibles, ce qui put entrainer des confusions entre le matériel contenu daus le sol encaissant al celui du comblement du silo. D'autre part, les embouchures se sont trouries usées et dargies. On s'en aperegoit car leur diamitre est beaucoup plus etroit lorsqu'elles furent creusies dans un matériau dur, par exemple dans un sol de tuileau. Des dements de couverture se reneonlrent dans lepaisseur du comblement, souvent vers le fond. Ce sont des dalles plates ou des lauses, dont le petit module peut justement siexpliquer par l'étroitesse des ouvertures - - $0^{\mathrm{m}} 45$ - conservées dans les betons. l.e comblement est souvent cendreux, à cause de la fonction de dépotoir que prennent ces silos a la fin de leur période d'utilisation. ()n ne sait pas déterminer combien de temps celle-ci pouvait durer. la repartition, le plus souvent régulière et sans recoupement à l'intéricur des pieces, implique sans doute que les recreusements ne s'imposent pas fri-

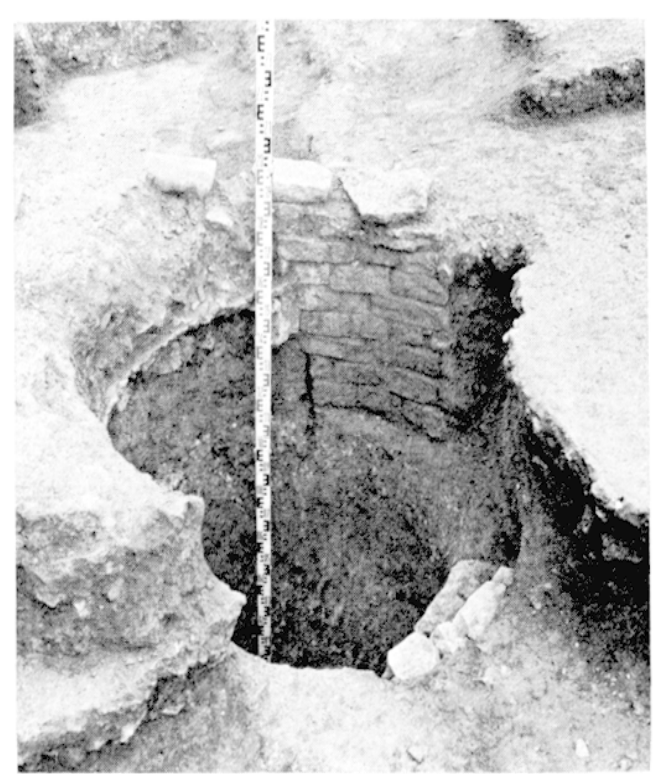

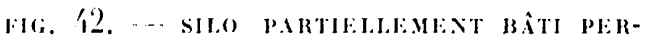

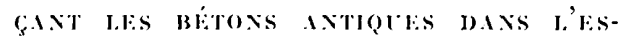
P.IC: 仡 quemment pendant le temps que dure le bàtiment. Mais les silos dattut-ils de son origine? Rién n'est évidemment moins sûr.

On notera aussi que ces silos se répartissent normalement soit a l'intérieur de piéces, soit à l'exterieur, ce qui est de constatation courante. Ici, un certain nombre d'entre cux est aligné le long de la façade méridionale, sur le tracé de l'ancien decumanus. Cela en ferait supposer l'appropriation par le détenteur des hatiments adjacents. Enfin, on remarquera que tout à fait à l'ouest, dans une zone non fouillée, qui explique un vide médian dans lá répartition, un silo a été repéré dans le prolongement du tracé du mur 51. Cela implique une modification du plan médiéval selon une direction non restituable dans cette zone un peu à part déjà en raison des orientations obliques des murs.

La répartition de ces silos nous conduit enfin à penser que les bâtiments à l'intérieur ou aupres desquels ils se trouvaient, dépendaient d'un ensemble. Dans ce cadre ils assumaient une fonction particulière liée à la vie quotidienne ou à l'activité économique, à la différence de l'espace central, la cour, ou des autres ailes qui répondaient à d'autres fonctions. Celte notion d'appartenance à un ensemble, que nous avons cherché déjà à rendre sensible lors de la description du mur 66 paraît indispensable à garder en mémoire au moment d'aborder les problèmes de datation et d'interprétation.

\section{e) Datation et interprétalion.}

La transformation décrite ci-dessus consiste donc en l'agrandissement d'un espace ouvert, d'une cour, au cour d'une insula antique, autour de laquelle se répartissaient des bâtiments, au moins au sud et à l'est, peut-être au nord. Du nord au sud, la distance est portée de 9 ou 1.1 mètres à $16^{\mathrm{m}} \mathrm{j} 0$. L'aile orientale est, pour sa part, reculée de 12 mètres, ce qui représente un gain considérable.

Mais précisément, s'agit-il d'un véritable accroissement de surface? Dans l'état antique, la cour de l'insula se poursuivait sans doute vers l'ouest. Elle pouvait s’ètendre jusqu à confronter une aile adjacente au cardo bordant le forum. Or, dans le nouvel ètat, la cour connaît une limite occidentale, un mur de bâtiment, ou une clòture. Il n'en a été retrouvé qu'un court tronçon, au sud-ouest du site, au pied de l'aile ouest du palais actuel. Lié à la terre, il repose sur le béton antique de l'espace I. C’est, à l'exception du mur 63, le seul vestige faisant saillie au nord de l'alignement du mur 71. Testige limité, de $1 \mathrm{~m} 20$ de long dont seul le parement occidental était conservé - 


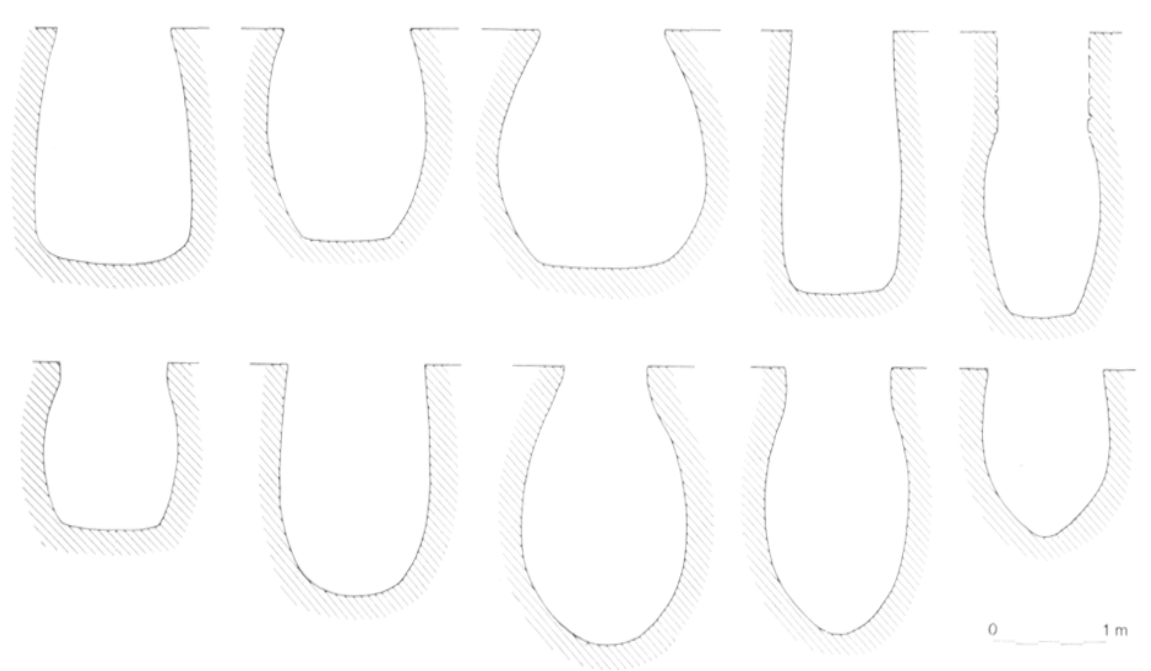

FIG. 13, - PROFIIS DE SIIOS

mais vestige important : il permet de mieux délimiter la nouvelle cour : de $16^{\mathrm{m}} \mathrm{j0}$ de large du nord au sud, clle avait 21 mètres de longueur d'est en ouest, soit une superficie proche de 350 mètres carrés.

De quelles possibilités dispose-t-on pour dater l'aménagement de ce nouvel espace et les restaurations qui l'accompagnent? On rappellera seulement la difficulté que l'on rencontre actuellement pour établir des repères chronologiques précis en se fondant sur le matériel archéologique et en tout premier lieu sur des productions de céramique du haut Moyen Age. Jusqu'à la genèse d'un Moyen Age plus «industriel » en ce domaine, les possibilités de datations offertes restent insatisfaisantes, compte tenu des types - sauf exception - mal différenciables de la poterie à pâte grise, qui est le fossile directeur essentiel des dark ages provençaux (55).

La difficulté que l'on rencontre dans l'interprétation du matériel numismatique est identique à celle qu'offre le matériel céramique. A la circulation prolongée des espèces antiques pendant le haut Mloyen Age, correspond à une date un peu plus tardive, à la fin $d u x_{1}{ }^{e}$ siècle, pendant le $x_{I I}{ }^{e}$ siècle et une partic du xin ${ }^{\mathrm{e}}$ siècle, la frappe et l'usage de types immobilisés. On peut donc envier la précision - à quelques années près - à laquelle permettent souvent de parvenir les repères antiques avec un même matériel.

Pour dater le moment où apparaît le nouveau dessin de la cour, le matériel céramique contenu dans les remblais, en particulier dans les espaces $\mathrm{V}$, ne donne donc que des indications sommaires. Il s'agit essentiellement de céramique antique et de quelques fragments de céramique commune médiévale à pâte grise, fragments de panses impossible à rattacher à des formes caractéristiques, sauf deux fragments porteurs d'un décor de roulette étroite et fine, bien connu sur les poteries de type pégau (56). Ces remblais pauvres portent des sols dont le dernier temps d'occupation se situe encore dans la période de production exclusive de céramique à pâte grise pour les récipients d'utilisation habituelle (fig. 45), formes fermées, pégaus à fond plat et pots à fond bombé, avec parfois des décors à la roulette. Parmi ces ustensiles qui constituent l'immense majorité des découvertes, se distinguent quelques rares fragments de marmite à anses horizontales, marqués sous le rebord plat et sur la panse d'un décor imprimé dans la pâte grâce à une molette.

Les quelques éléments de céramique plus fine et plus colorée se comptent à l'unité. Ils appartiennent à des poteries d'origine étrangère mal identifiée à pâte blanche portant une glaçure monochrome verte. Enfin ont été récoltés dans ces niveaux quelques tessons appartenant à des poteries d'un type inconnu jusqu'ici, modelées ct. non tournées, formes ouvertes à parois épaisses et à glaçure plombifère sur pâte rouge.

Ce faciès, à l'exception des marmites, se trouve dans l'espace VIII, à l'intérieur du remblai de la fosse. Il se trouve identique aussi dans les sols du passage VII, à partir de la cote 204, et dans la pièce V c (sol 4), à la même cote. Dans les espaces III, il inclut les marmites grises à anses horizontales, qui sont aussi représentées dans les sols stratifiés de l'espace VIII. Ce matériel est tout à fait comparable à celui qui caractérise les ensembles périodisés A1-A2 dans la fouille d'habitat de Rougiers. Il marque le tout début de la période d'activité sur ce site de référence, c'est-à-dire a fin du xir ${ }^{\mathrm{e}}$ siècle et le commencement du xinl ${ }^{\mathrm{e}}$ siècle (57). 


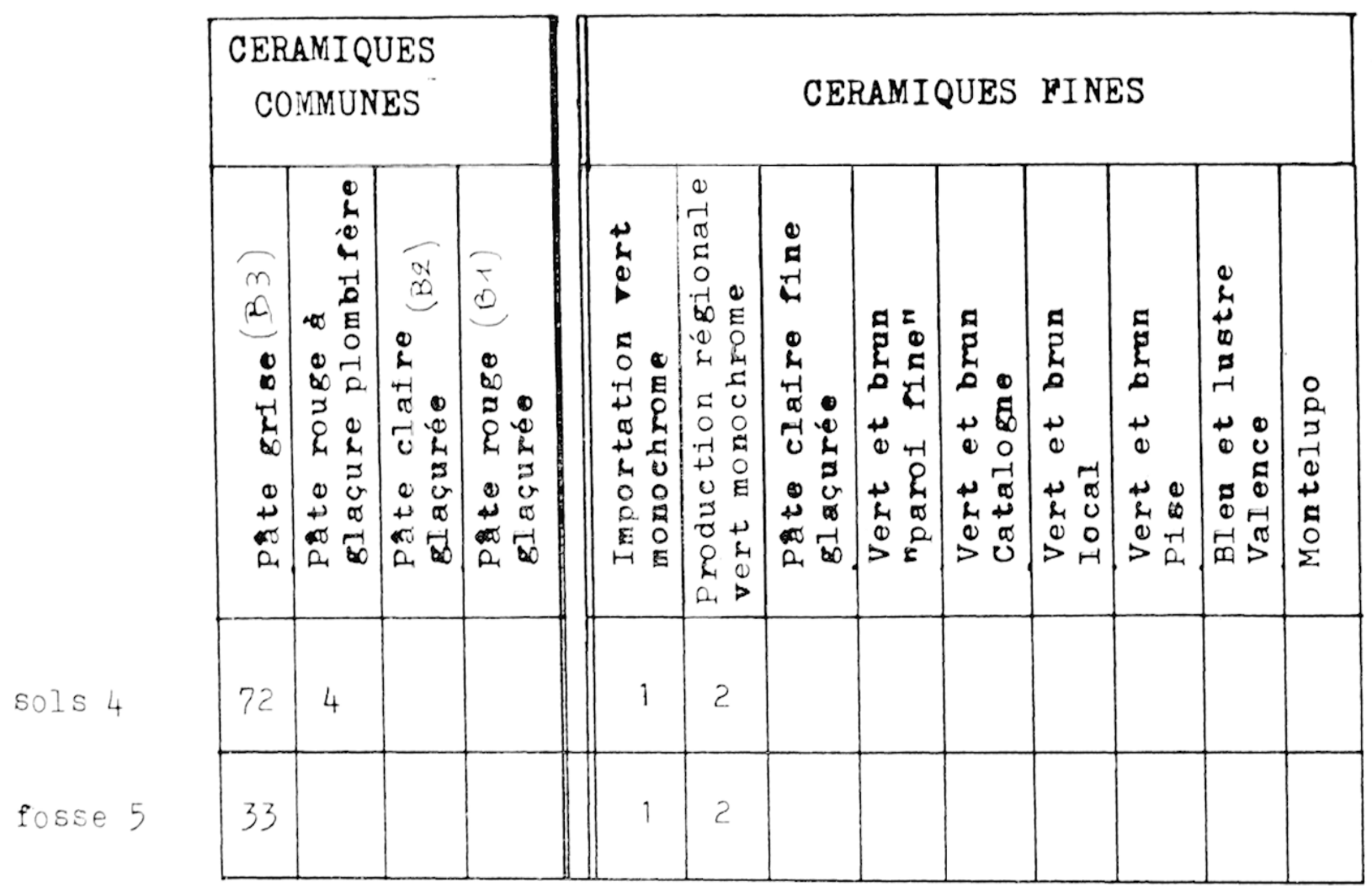

Tabifiu X. Répartition des céramiques dans les niveaux romans de l'espace VIII. (N.-B. La classification des céramiques communes correspond à celle adoptée par G. Démians d'Archimbaud, Les fouilles de Rougiers, op. cit.).

Les indices que domnent les monnaies trouvées dans ces sols correspondent bien à ces dates. Les monnaies 34 et 35 sont des frappes d'Aphonse I et d'Alphonse II d'Aragon. L'obole 34 a été découverte dans les sols stratifiés de l'espace VIII, et le royal coronat 35 sur le sol cendreux de l'espace V a à la cote 203,95. Le denier Guillermin 36 provient également de l'occupation de l'espace VIII.

La même impression se dégage de l'étude des lots de céramique et des monnaies récoltés dans la fouille des silos. La cessation de l'utilisation de la plupart s'effectue aussi avant le moment où se répandent les céramiques communes vernissées à pâte claire. C'est ce dont témoigne la représentation sur le plan de répartition (fig. 36). Les comblements contenaient done, pour la grande majorité d'entre eux, un matériel fait de céramique à pâte grise, ou dans certains cas, d'un ou deux fragments de céramique fine à couverte monochrome verte (58). Là encore, les monnaies confirment l'homogénéité chronologique de ce faciès, avec un fragment de denier du Puy au type dégénéré $n^{0}$ 42), deux oboles de Melgueil $\left(\mathrm{n}^{\mathrm{os}} 39\right.$ et 40) et un denier du même atelier ( $\left.\mathrm{n}^{0} 38\right)$.

Le matériel trouvé dans ces comblements de silos et ces sols marque la période finale d'une utilisation avant des modifications d'ensemble qui s'accompagnent d'une recharge des niveaux d'occupation. C'est donc bien à l'époque romane au sens large que l'on peut attribuer le nouveau dessin apporté à la cour de l'insula. C'est donc aussi jusqu'ì cette période, le $\mathrm{xul}^{\mathrm{e}}$ siècle, que les constructions antiques, avec des modifications de détail, ont donné leur cadre à l'organisation de l'espace et de la vie quotidienne. Lne telle conservation implique à nos yeux une continuité d'utilisation sans rupture, sans l'ałandon propice aux remaniements plus radicaux ou aux destructions motivées par le besoin de matériaux. Cette continuité archéologique implique une pérennité de la vie urbaine.

Quel est le paysage roman qui succède? Au sud de la cour, une aile méridionale a été reconstruite. Le bâtiment était moins profond que celui qui le précédait : il n'avait que 13 mètres de largeur du nord au sud. En dépit du retrécissement operé, on s'est aperçu que la transformation n'avait consisté qu'en une adaptation étroite au plan antique, en un véritable démarquage de l'organisation de l'espace. Une telle affirmation de continuité spatiale ou architecturale traduit une continuité d'affectation. Or, dans la répartition antique, c'était cette région de l'insula qui, dans les limites de la fouille, était la plus riche en volumes bâtis. Il nous semble que la reproduction du plan antique par la construction romane s'expliquerait mal si l'on n'avait affaire qu'à des pièces d'intérêt secon- 


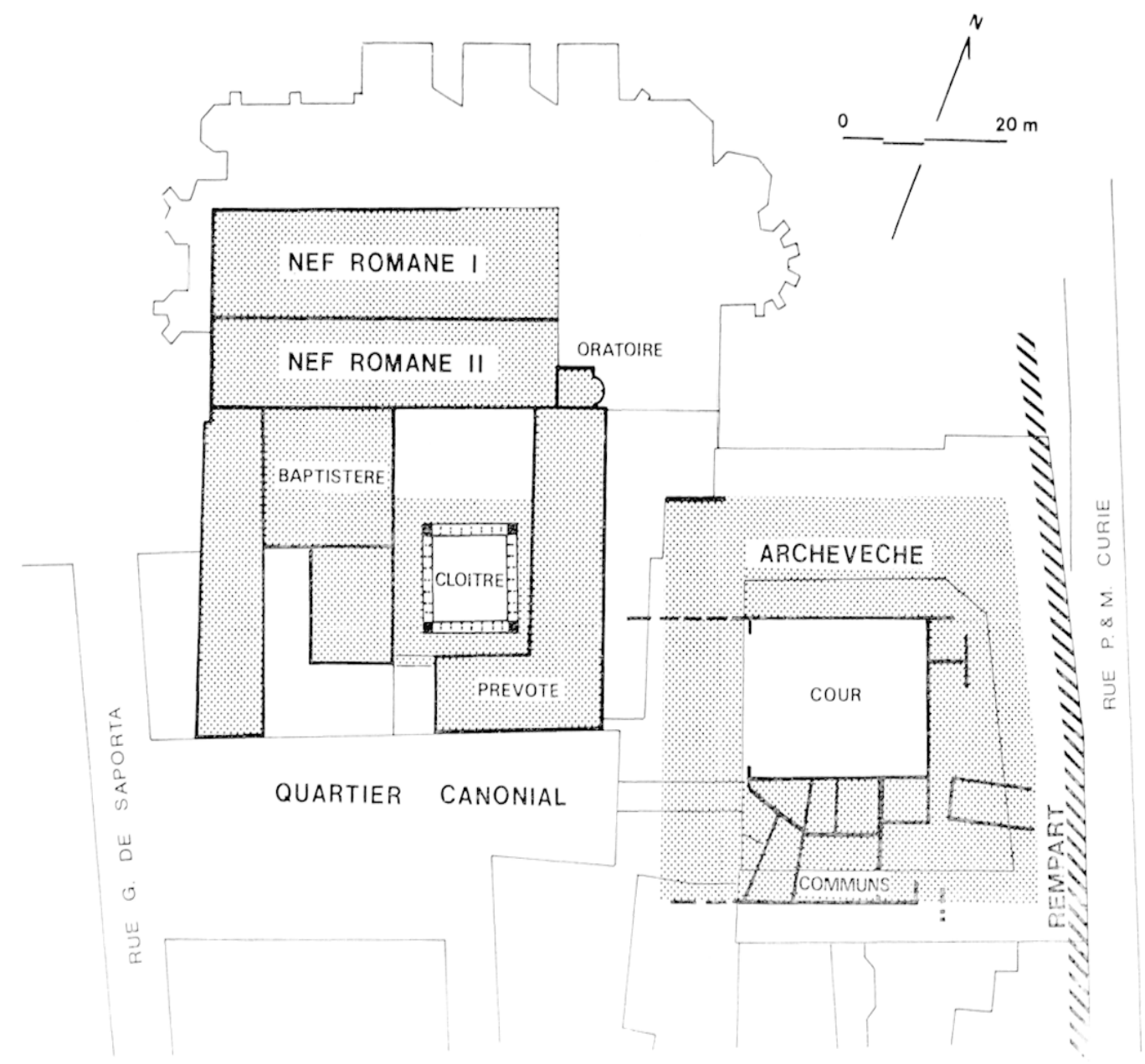

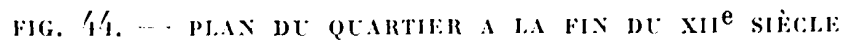

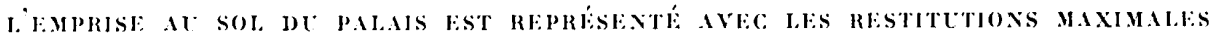

daire. Aussi penserait-on volontiers que l'aile sud aurait pu être, dans un premier temps, une maison avec des dépendances organisées autour de la salle $V^{\circ}$ e, la plus vaste. Dans un deuxième temps, l'ensemble aurait évolué de la fonction d'habitation vers celle de grange et de réserve exchusivement.

Pour l'aile oricntale romane, et son organisation intérieure, nous ne pouvons que déplorer la pauveté de la documontation archéologique. Remarquons toutefois que c'est vers elle que débouchait le passage conservé, sous la forme d'une ruxlle, ou même d'un cheminement couvert. Máas pourquoi la déviation du tracé de lancien portique s'est-elle effectuée vers elle plutòt que vers la cour? Y aurait-il eu là un espace ouvert, galerie ou promenoir? (Quant à la restitution d'une aile nord romane, nous avons déjà fait part de nos perplexités. Quoi quil en soit, l'interprétation proposée doit prendre en consideration limpression qui s'est petit à petit dégagie, selon laquelle la fouille a fait retrouver une partie d'un bâtiment dont le caractère monumental ne peut être nié. Il faut entendre par là que l'on n’a pas affaire à un ensemble de cellules ou d'habitats juxtaposés. Au cours de son remodelage, l'îlot a été traití selon un dessin d'ensemble, avec une répartition spatiale des fonctions selon les ailes. C'est ce. qüindiquent en particulier les silos, si importants de ce point de vue (59).

L'aménagement roman ne prend alors son sens qu'en fonction des modifications qui affectent à la même époque le quartier, cét-à-dire le groupe épiscopal et ses abords fig. 4/4. R. Guild a retracé cette évolution dans un travail resté injustement confidentiel $(60)$. Il a montré comment la restauration du baptistère d'origine antique a été le point de départ, a la fin du xie siècle, d un mouvement de rénovation qui śaffirme dans le courant du siècle suivant, dabord avec la reconstruction de la nef épiscopale Sainte-Marie et celle de l'oratoire canonial Saint: Sauveur. Cet effort monumental était consacré par la restauration de la dignité archiépiscopale dès 1102 et la tenue d'un premier concile métropolitain en 1112. La seconde moitie du xule siècle connaissait le renforcement de la monumentalité nouvelle donnec au quartier. La construction de la nel canoniale, au sud, dans le prolongement 
de l'oratoire du Sauveur, réaffirmait sans doute le schéma de l’église double du groupe épiscopal primitif. Au sud du groupe encore, l'organisation d'un cadre de vie canonial s'opérait selon des modes identiques, c'est-à-dire en conservant la mémoire des lignes directrices héritées de l'Antiquité et en respectant l'esprit d'ordre dans ce qui était innovation. Ces termes justifient d'ailleurs assez bien la notion de Renaissance appliquée à cette période du xne siècle. R. Guild a également montré comment, sur le tracé de l'ancien cardo bordant à l'est le forum, de manière adjacente à l'oratoire du Sauveur, était élevé un grand bâtiment au rez-de-chausséc voûté, destiné aux chanoines, bâtiment de 22 mètres de long au moins et de 8 mètres de large. Son mur occidental étail fondé pour une part sur l'ancien mur périmétral du forum, puis dans l'alignement de celui-ci. Son mur oriental repremait le tracé du mur bordant le cardo à l'est. Dans l'organisation ultérieure cette vaste construction allait devenir l'aile est du cloître. Fille est attribuée à une date un peu postérieure à 1060). Ja bâtisse fut profondément reprise à une époque qui suivit la construction des galeries du cloître; aussi nen comnât-on pas la limite méridionale primitive. A l'origine, elle fut implantée sur la largeur de l'ancien cardo. Eille affectait done dans son plan une bonne partie d'une aile oceidentale qu'il n'est pas abusif de restituer à cette insula antique dont l'extrimité orientale subsistait encore, peu retouchée, à l'époque romane, dans la Cour de l'acturl archevêché. T'oute cette partie fouillée de l'insula a fait l'objet, à cette époque, d'un réanénagement global, et comme nous lavons vu, d'un traitement d'ensemble de l'espace. Or, à chaque extrémité de l'insula, à des périodes assez proches, se produisent, scmble-t-il, des agrandissements symétriques réalisés aux dépens des anciens espaces publics, sur le cardo longreant le fortum a l'ouest, sur le portique et le boulesard à l'est. Cette symétrie s'expliquerait assez bien dans un plan gineral destiné à élargir un espace initial arant sa division matérialisée par le troncon du mur ś. Serait-ee un simple mur de limite ou celui de la façade d'un bâtiment implanté approximativement par lo milieu de l'ancionne cour? Coette question rejoint celle qui a été posée pour la restitution d'une aile au nord de la cour romane. La réponse est aussi difficile et l'on ne peut qu'hésiter. Cependant, après avoir penche en faveur de lidé simple de limite, il faut dire que celle du bâtiment est séduisante. Séduisante d'abord en raison des continuites : ce mur \$́́ est presque a l'aplomb de la façade oecidentale actuelle donnant sur la cour, ce qui lui a dailleurs valu de disparaître presque complètement, et ainsi de risquer de nous échapper. Mais l'idée est séduisante aussi en fonction de ce qui se passe dans la partie nord-ouest du site. On expliquerait mal autrement limplantation médiévale romane puis gothique, de l'extrémité occidentale de l'actuelle aile nord, empiétant sur la division opérée par le mur 8'. Fin revanche, cette implantation s'explique mieux si l'on peut compter avec la largeur d'un bâtiment développé an arrière de ce nur. Ce sont done deux raisons pour ne pas écarter la possibilité d'une aile occidentale sous l’aile actuelle. (Quoi qu'il en soit, on voit comment la Cour de l'Archevêché d'une part, et l'actuelle cour de la prévôté de l'autre, tirent leur origine du partage vraisemblable de la cour centrale d une insula du ${ }^{\mathrm{e}}{ }^{\mathrm{e}}$ siècle.

Il serait évidemment tentant de refermer l'espace au nord par une aile bâtic sur l'ancien decumanus, de la même manière que l'aile orientale du clổtre occupait le cardo. Les vestiges dans le mur nord de la travée occidentale de cette aile en sont la trace. Le mur est plus épais en cet endroit et une corniche très saillante pourrait marquer un départ de voûte en berceau maintenant disparue. Cette architecture renverrait à celle des longs espaces romans voûtés en berceau, que ce soit celui qui apparaît au $x_{1}{ }^{\mathrm{e}}$ siècle à l'est du cloître, transformé ou allongé plus tard vers le sud, ou encore le bâtiment occidental à bossages qui Jonge l'ancien cardo _- l'actuelle rue Gaston de Saporta -... à la fin du xir e siècle.

Semblable division accompagnée d'une réaffirmation de l'ensemble monumental issu de l'insula antique, avec division de part et d'autre d'un mur ou d'un bâtiment, suggère un grand projet architectural. En raison de tout ce qui a été dit, on proposera d'identifier la partie orientale de l'insula, avant et après sa transformation, avec la domus de l'évêque. Dans la division qui s'opère, ce serait donc lui qui en aurait la meilleure part. De l'autre côté s'organise le corps canonial qui dispose d'une partie de l'ancien forum, et la séparation pourrait être la marque d'une autonomie acquise autour de la personne du prévôt. Chronologiquement, on ne se situe peut être pas très loin de l'époque où l'archevêque Pierre II cède au prévôt Benoît edificia domorum et loca Ricardi Bernoini et Odonis canonici filii sui quae circa claustrum Beatae Mariae Virginis Aquensis sedis, inter domum nostram et ejusdem Genitricis Dei ecclesiam fundata sunt (61), maisons qui pourraient correspondre encore à des pièces subsistantes dans la partie occidentale de l'insula ancienne, sous dépendance canoniale.

Vers l'est, l'occupation systématique de l'espace aux dépens du portique et du boulevard antique dans le courant $\mathrm{du} \mathrm{xII}^{\mathrm{e}}$ siècle à coup sûr, rappelle que ces surfaces se situent alors à proximité du lieu où passe le rempart médiéval (62), sur le tracé du rempart antique peut-être. Il est possible aussi d'imaginer que le recul tienne compte de la présence de ce butoir.

On ne connaît pas, en effet, la profondeur de l'aile orientale. Comme l'occupation de l'espace public ne pose pas de problème particulier à l'évêque ou l'archevêque dans le Bourg Saint-Sauveur, on verrait très bien cette aile orientale s'étendre jusqu'au pied du rempart qui était sur le tracé de l'actuelle rue Pierre-et-Mlarie-Curie, ou laisser seulement la place pour une circulation. 
Cette disposition du palais, adjacente au rempart, sans l'intermédiaire de jardins comme on l'a cru parfois, rappelle de nombreuses dispositions identiques. Le palais archiépiscopal d'Aix respecte ici une topographie habituelle dans laquelle ce batiment est adjacent au rempart de ville.

Organisé autour d'une cour centrale d'un peu moins de 350 mètres carrés de superficie, il s'apparente régionalement à d'autres résidences épiscopales de mème période ou un peu plus tardives. Ainsi pense-t-on à celle de Fréjus qui se présente au xır e siècle autour d'une cour de 250 mètres carrés environ, limitée semble-t-il par un portique sur les faces ouest et sud (63). Mais la cour autour de laquelle s'itendent les ailes du palais d'Aix est beaucoup plus petite que celle du palais d'Arles dont l'aile sud s'appuie également au rempart médiéval. Selon la restitution proposée par C. Sintès, elle occupe environ 1.200 mètres carrés, et la différence est d'ailleurs significative" de l'importance respective des sièges, tt des villes (64).

Reste à évoquer une question qui a beaucoup agité les historiens d'Aix. Pour tous les auteurs, avant que ne paraisse l'étude de J. Pourrière (05), aucun palais épiscopal ne pouvait avoir existé à l'emplacement actuel avant le second quart du xive siècle, voire la fin du premier quart du siècle suivant. On situait alors le palais antérieur dans la partie occidentale de la ville antique, auprès de l'endroit où l'on plaçait aussi le lieu de la première cathédrale avant un transfert sur le site actuel. Rappelons que ce transfert n'est pas démontré te que les fouilles récentes ont attesté que la cathédrale s’élevait déja au nord de la ville vers $500(66)$. Il paraîtrait done étonnant qu'il y ait eu une si longue dissociation entre la domus de l'évêque et le groupe épiscopal. J. Pourrière montra que dès la fin du $\mathrm{xI}_{1} \mathrm{e}$ siècle, ne serait-ce que selon le texte de Pierre II cité plus haut, la domus episcopi avait de bonnes chances de se trouver à l'emplacement de l'actuel archevêché. Le fait est qu'il y a eu dissociation tcmporaire entre la résidence de l'évêque et le lieu où s'exerçait l'administration du diocèse. La villa des Tours apparaît en effet comme refuge quand les circonstances politiques l'exigent, ou quand un archevêque comme Guy de Fos s'installe dans ce quartier, où sa famille est abondamment possessionnée (67).

En dehors de ces accidents, il n'y a pas lieu de soupçonner que la résidence normale de l'évêque, puis de l'archevêque n’ait pas été auprès de sa cathédrale.

La seconde moitié du xiI ${ }^{\mathbf{e}}$ siècle semble en effet être le moment où l'archevêque prend quelques distances vis-à-vis du Bourg Saint-Sauveur. Cela se concrétise par l'acte de 1185 par lequel le chapitre - et non l'archevêque, reçoit d'Alphonse d'Aragon la juridiction sur le Bourg. Cela engagerait à fixer la genèse du palais roman à la fin du $x_{I}{ }^{e}$ siècle ou dans la première moitié du $x_{I I}{ }^{e}$ siècle. Les constructions en paraissent médiocres par rapport aux bâtiments canoniaux qui s'élèvent dans le courant du xiı siècle. Pierre II habitait-il encore l'insula ou quelque part dans cette nouvelle résidence? En fonction des continuités maintes fois soulignées, il est probable de toutes manières que la domus episcopi occupait l'insula depuis un certain temps. On ne sait que peu de choses du cadre de vie résidentiel méridional au tournant du $\mathrm{xi}^{\mathrm{e}}$ et $\mathrm{du} \mathrm{x}_{11} \mathrm{e}^{\mathrm{e}}$ siècle en dehors de quelques exemples ruraux. Fin fonction de la grande rusticité ambiante, et des observations faites plus haut, on peut se demander si l'habitation de l'évêque à cette époque n'aurait pas pu se trouver dans la pièce $V$ c et ses dépendances, avant la reconversion de l'ensemble de l'aile sud à des fins utilitaires.

\section{II. - LF PAIAIS GOTHIQLF}

On l'a vu, le débat historique sur les origines du palais archiépiscopal s'est trouvé compliqué et faussé. Le respect porté aux auteurs du $x_{n 1}{ }^{e}$ siècle (68) a, semble-t-il, empêché que ne soient étudiés de manière critique les vestiges gothiques encore présents dans le palais actuel. Il est vrai qu'avant la rénovation récente, ils étaient fort encombrés et peu accessibles. Ainsi, pour la fin du Moyen Age, à la différence de la période précédente, l'étude archéologique peut prendre pour objet non seulement le sol et ce qu'il contient mais aussi les élévations. L'intérêt est évidemment plus grand lorsque l'on peut utiliser les deux types d'approche.

a) Le renforcement du schéma roman au sud-est et la préservation de l'ancien passage.

\section{1) Discription.}

Il paraît préférable de commencer la description du palais à l'époque gothique par la partie qui nous est la plus familière, même s'il ne s'agit pas de la plus importante dans le dessin d'ensemble. Il s'y marque des reprises inscrites dans une continuité qui affecte encore le bâtiment moderne récemment reculé.

La plus importante concerne l'angle formé par les ailes sud et est. Une restructuration notable des volumes a lieu à l'est du passage. Ceux-ci étaient déjà privilégiés dans l'état préroman, mais assez difficiles à définir. A l'époque romane, les transformations apportées alors que le passage était dévié n'ont pas laissé non plus une image bien précise. A l'époque suivante, l'importance particulière de ce secteur est réaffirmée. Malheureusement, l'édifice qui résulta de la reconstruction ne se définit toujours pas de manière entièrement satisfaisante (fig. 45). 


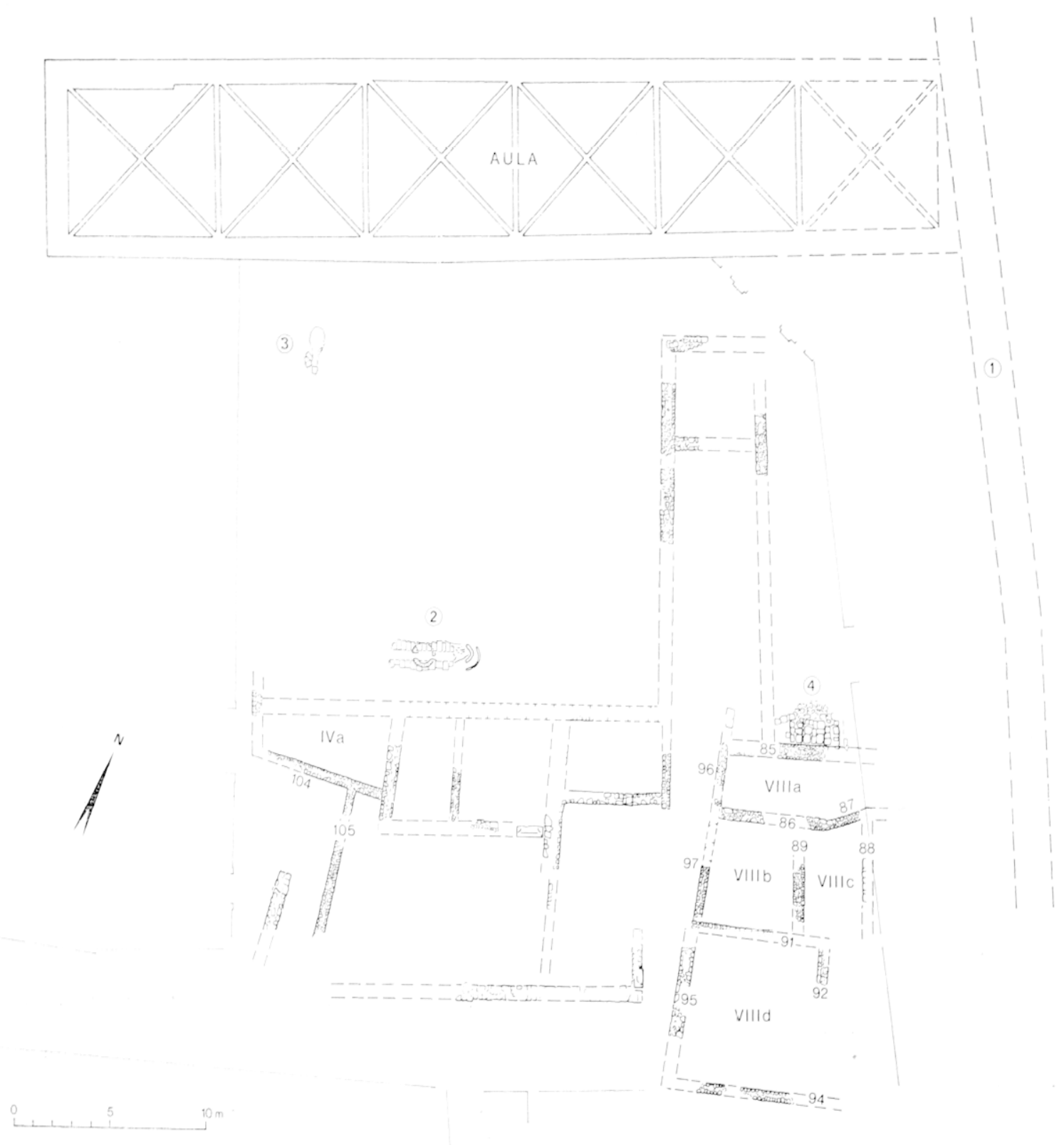

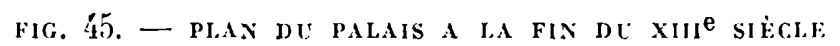

11 rempart; 2) atelier du fondeur de cloche; 31 four de bronzier; 4 l cheminie.

Trois murs d'orientation est-ouest, parallèles entre eux font leur apparition dans cette zone. Ils ont le même aspect, sont bâtis en mœllons très réguliers, parfaitement assisés, avec cette fois du mortier pour liaison. Le mur 85 est le plus septentrional. On peut le considérer cornme une résurgence du mur 66, le mur au support engagé qui est alors détruit. La nouvelle élévation est implantée à $0^{\mathrm{m}} 60$ en retrait, vers le sud, selon un alignement strictement identique (fig. 46). Le mur 85) est lié au mortier depuis ses niveaux de fondation enfouis à la cote 202,75 dans l'argile naturelle. Il est épais de $0 \mathrm{~m} 70$ et peut supporter une élévation à étage; déjà, on le sait, le mur 66 pouvait, au même endroit, être à la base d'une telle élévation, plus médiocre.

L.e mur 85 séparait deux salles. Celle qui se trouvait au nord dépendait de l'aile orientale du palais. Son niveau d'occupation est situé beaucoup plus haut que les niveaux romans. Partout les transformations s'accompagnèrent d'une recharge des sols. Ici la terre battue de la pièce était à la cote 204,15. Immédiatement sur le sol étaient posées les dalles rougies et rongées par la chaleur d'une cheminée adossée au mur 85. L’âtre formait un carré de 1 mètre de côté (fig. 47). Il était limité par la base des jambages. Chronologiquement, cette cheminée constitue le premier fover construit ou non, intérieur ou extérieur - retrouvé sur le site. Elle se siluait près de l'angle de la pièce dont le mur occidental, tout proche, prolongeait 


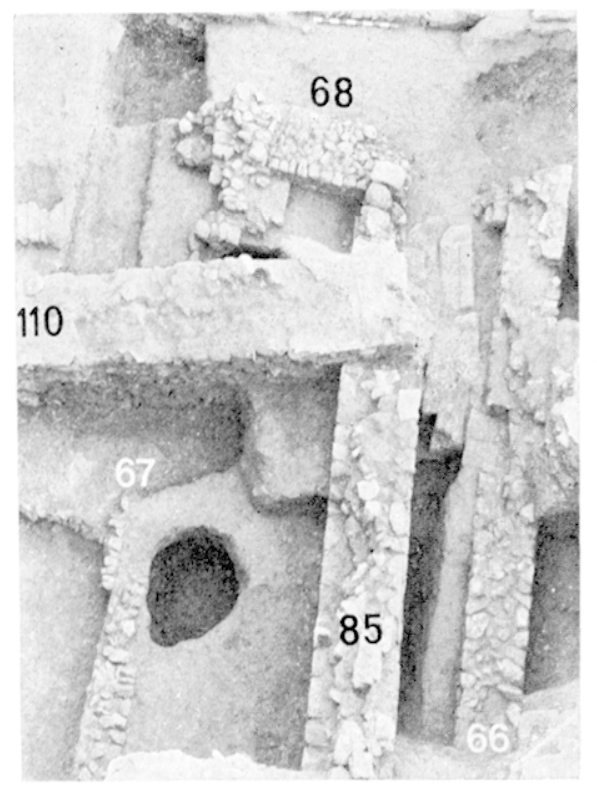

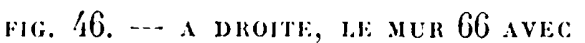
SON SUPPORT EXgaGé. PARALIĖ.FMENT A SA GAUCHE, LE MUR $8 j$ QUi I.UI FST SUBSTITUÉ AU XIII $\mathrm{e}$ SIÈCLF.

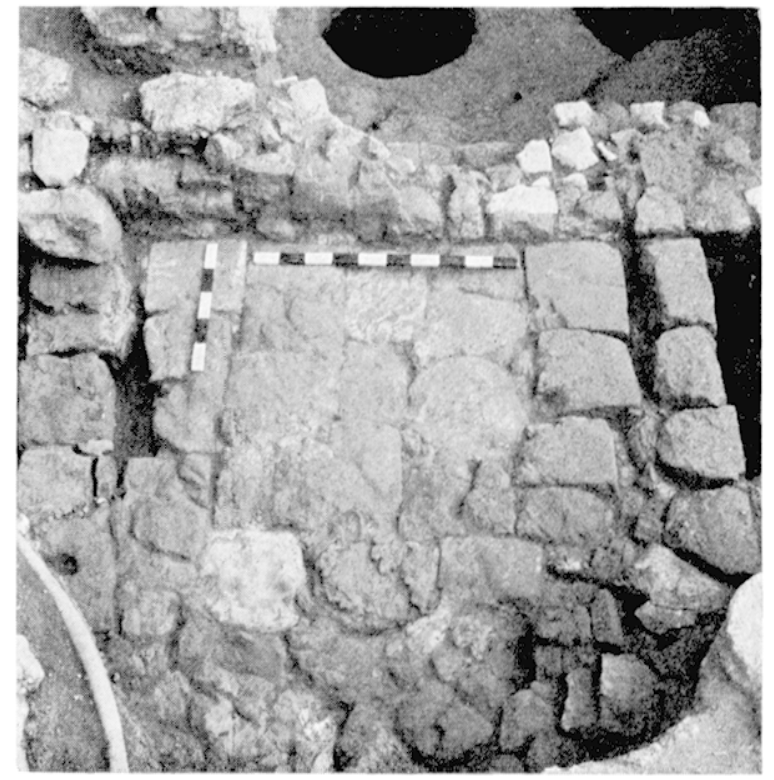

FIG. 47. - LA CHEMIXF́E ADOSSÉE AU MUR 8j

le mur 65, contemporain de l'âtre; mais le mur ouest était fondè à une cote plus élevée, sur les remblais dont l'accumulation avait accompagné la destruction du mur 66.

Dans cette situation, la cheminée devait être décentrée par rapport à l'axe de la pièce. On y verrait volontiers un espace ayant servi de cuisine, fonction que l'on retrouve à une date plus récente aussi dans l'aile orientale du palais. Mais la chronologie est longue, et il faut se méfier de continuités seulement apparentes : l'aile orientale a reçu d'autres fonctions au cours des temps.

Le mur 91 appartient au mềne système, avec toutefois une légère déviation. Il n'en est apparu qu'un tronçon long de $l_{\mathbf{m}}^{\mathrm{m}} 50$. I)es constructions datables du $\mathrm{xvI}^{\mathrm{e}}$ siècle, puis celle des nouvelles écuries de Charles de Vintimille, entre 1715 et 1722 , l'ont endommagé. lin particulier, au cours de cette dernière campagne de construction, le mur de façade de l'aile méridionale en a repris l'emplacement, à défaut de l'alignement précis. Est-ce un effet du hasard? Une reprise identique s'est produite dans le cas du"mur 9', exactement parallèle au sud, élément du même syvstème médiéval. les deux derniers murs, 91 et 94 , sont fondés à la cote 203,35. L'implantation de ce dernier consacre l'occupation définitive de l'ancien decumanus, que nous avons déjà décrit dans un état vraisemblable d'appropriation à cause de son occupation par les silos. Sur lui empiétait déjà le mur 70 .

Les extrémités orientales de ces trois murs sont indéterminées. Les constructions modernes en sont la cause, à des titres divers. lin revanche, leurs extrémités occidentales, et la limite du volume double qu'ils déterminent, posent le problème de la conservation du passage issu de l'ancien portique. Or ce passage était encore préservé. On peut s'assurer de sa trace même si, dans le détail, l'évolution des constructions est parfois malaisée à suivre. La continuité de son utilisation est marquée à l'est par un alignement de murs assez hétéroclites sur lesquels s'articulent les extrémités des murs 85,91 et $9{ }^{\prime}$, dont la qualité de construction tranche avec la rusticité de ceux qui ferment à l'ouest les volumes VIII. Parmi ceux-ci, le mur 96 forme un alignement précis, reprenant partiellement pour fondation celle du mur 68 , qui relève de l'état antérieur. Le mur 96 est prolongé au sud du mur 91, par le mur 95, plus large, mais bâti dans le même alignement. C'est ainsi que se trouve régularisée la paroi orientale du passage selon un tracé entièrement oblique par rapport à celui du portique. Il n'est peut-être pas nécessaire d'entrer davantage dans le détail de la description. Remarquons simplement que le niveau d'utilisation du passage s'établit alors au-dessus de la tranchée de récupération des pierres de fondation du mur du portique. C'est la raison pour la* quelle on peut penser que celui-ci subsistait encore à l'époque romane, supportant une élévation (fig. 41 et 48 ).

Les deux volumes majeurs de l'espace VIII furent recoupés par des cloisons plus légères, faites de murs liaisonnés à la terre. Parmi elles se trouve le mur 86, dont l'implantation est issue du désir de réaménager des espaces en tenant compte des volumes antérieurs. Par rapport à la cloison 67 , la nouvelle cloison 86 fut reportée un peu plus au sud, de même que le mur 85 avait été bâti au sud de l'ancien mur 66. Aussi, en dépit de la reconstruction, la surface précédente était-elle exactement conservée (fig. 37, 45 et 46). Mais ce déplacement n'affecte pas tout l'espace. 4 l'extrémité orientale du sondage, le mur 87 , en biais, vient rattacher le mur 86 à l'ancien alignement du mur 67 qui devait subsister plus à l'est. De telles continuités résultant du report des volumes ont été naguère observées, pour l'époque précédente, dans l'espace V.

Insistons enfin sur une observation effectuée sur les tranchées de fondation des murs dont les pierres ont été récupérées à cet endroit : le mur 86 venait bien buter contre le mur 96 , sans se prolonger au-delà, au travers du passage. Celui-ci demeurait donc bien utilisable, soit vers l'aile orientale encore, et par son intermédiaire peut-être vers la cour comme à l'époque 


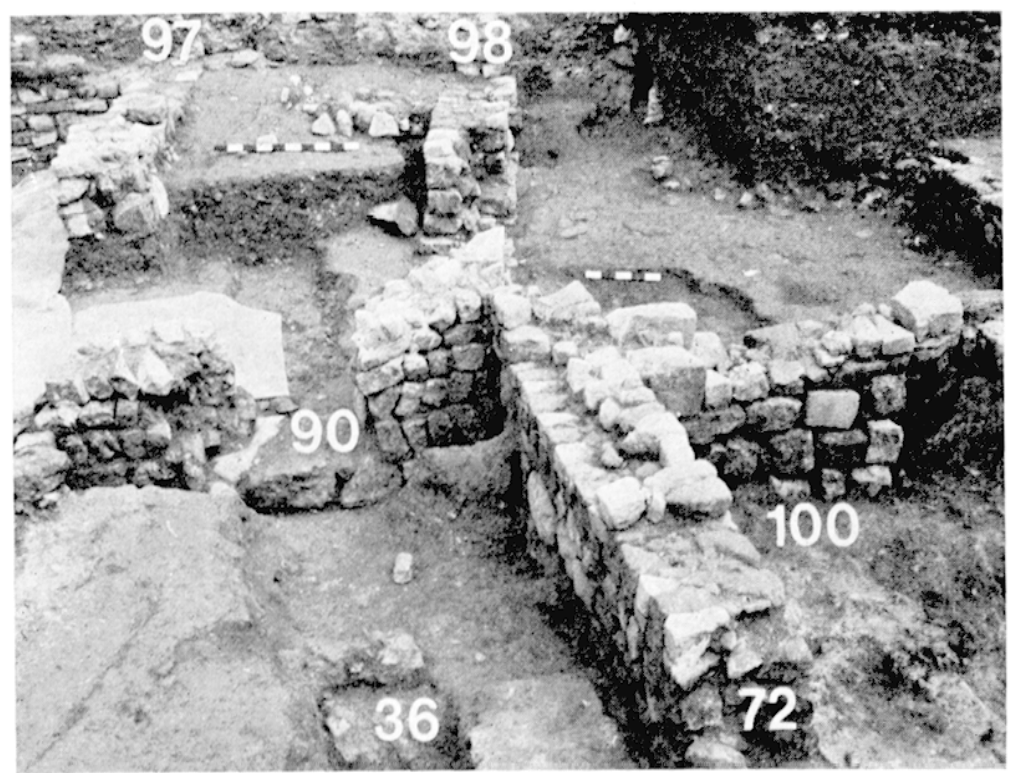

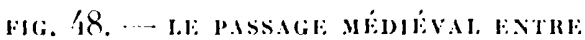
L.es ners 72 lat 96 pels 98 nT 97.

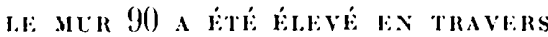

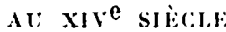

précédente, soit seulement pour desservir les pièces adjacentes au passage. Mais aucun seuil, aucun emplacement de porte n'a été trouvé qui permette de préciser le système de circulation.

\section{2) Datatron.}

En dépit de l'étroitesse du sondage au nord du mur 85, la fouille faite ici a été précieuse pour dater ces transformations. La netteté du sol de terre battue autour de la cheminée, la cheminée elle-même, recouvraient les volumes de remblais liés a la destruction du mur 66. Le décalque de l'espace VIII a, dans l'un et l'autre état, rend certaine une succession immédiate.

La modification se situe done encore dans la période de fabrication et d'usage presque exclusif de la céramique commune grise $(69)$.

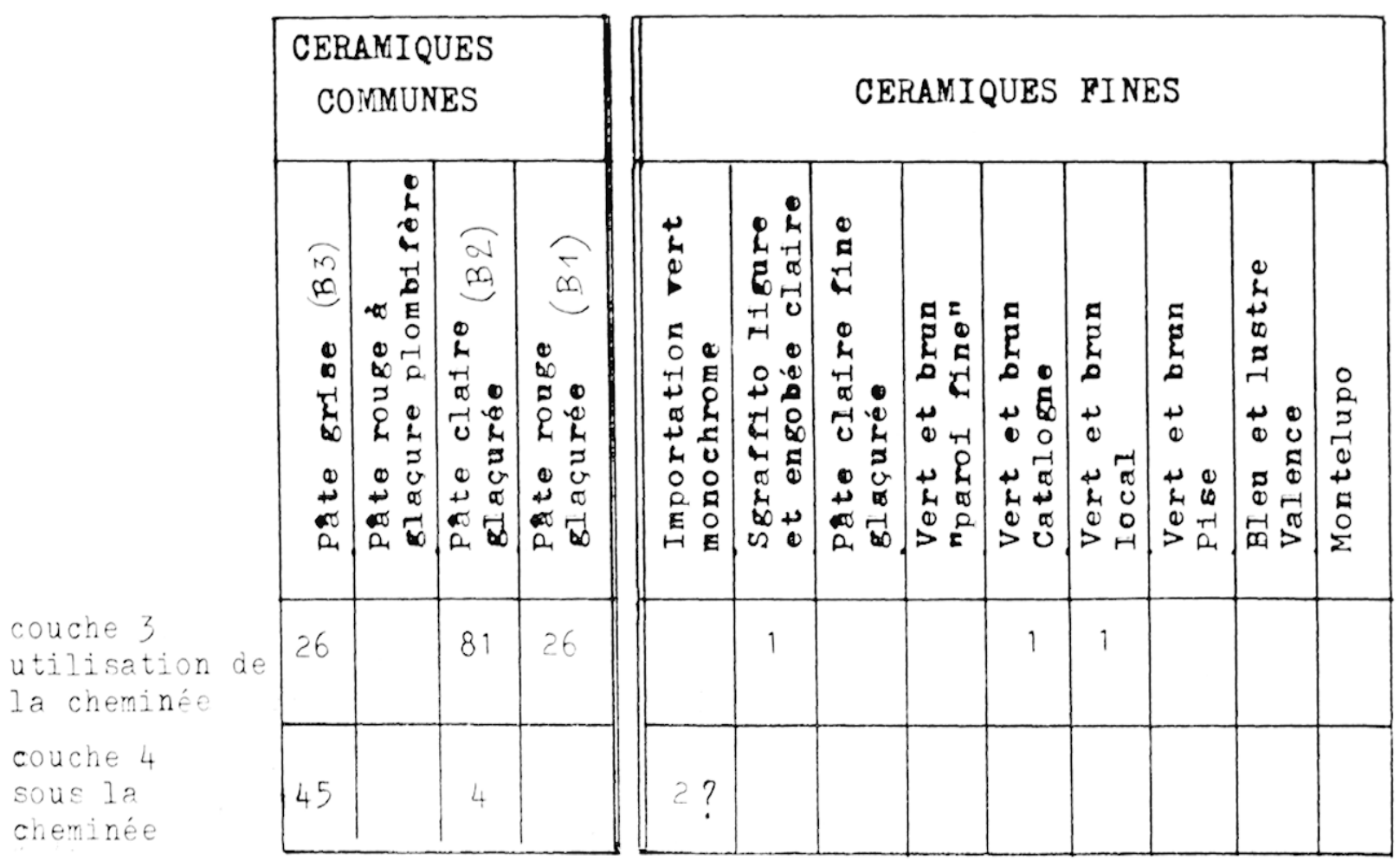

Tabifac XI. Datation de la construction et de l'utilisation de la cheminée de l'aile orientale d'après la céramique. 


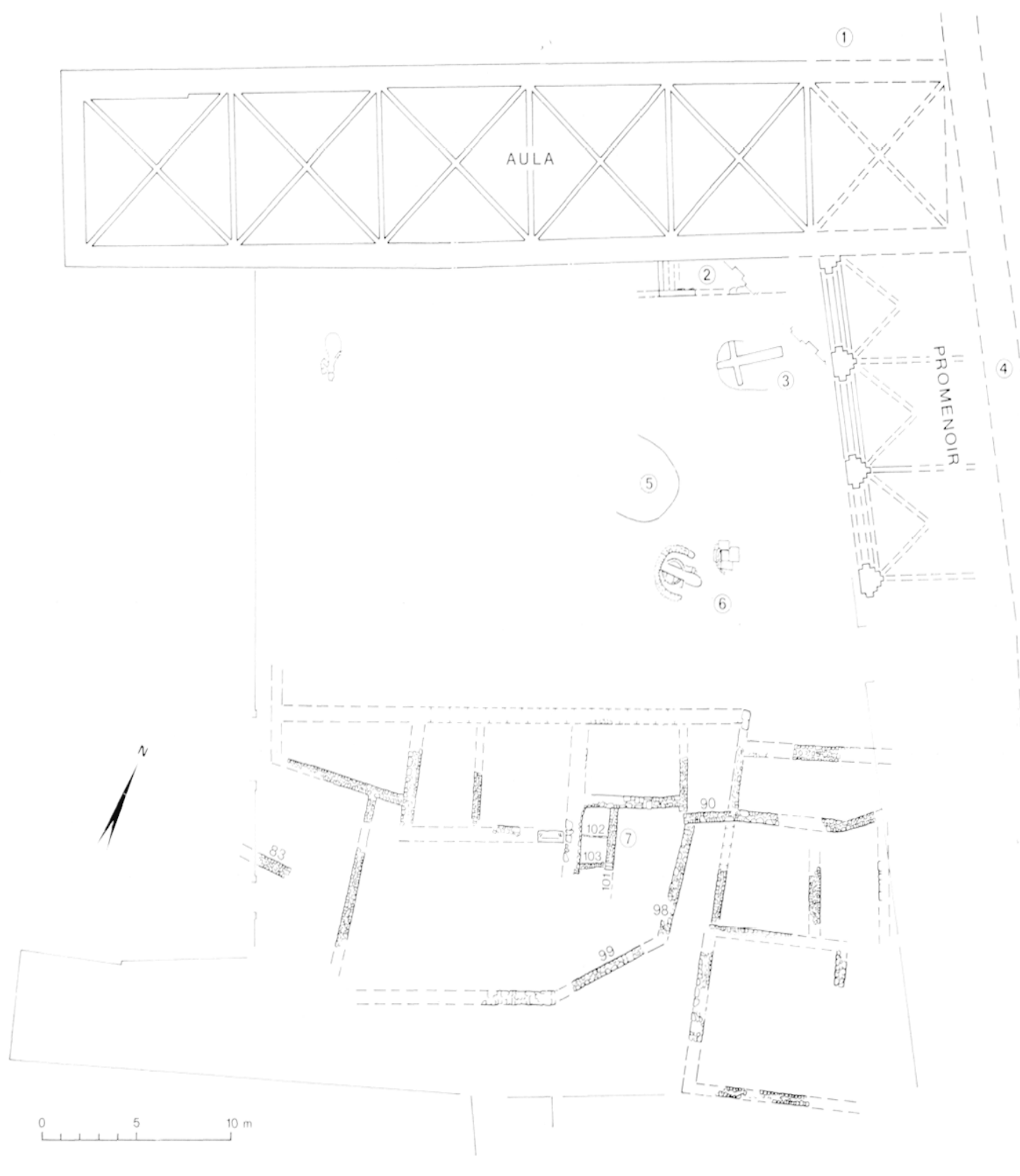

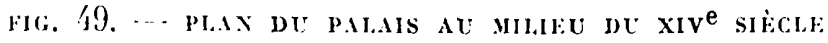

1) emplacement de la chapelle; 2) escalier droit ; 3) et 6) ateliers de fondeurs de cloches ; 4) rempart ; 5) four à chaux ; 7) silos maçonnés.

Lin indice monétaire concordant est donné par la découverte d'une obole de Plaisance d'un type immobilisé, en usage encore au $\mathbf{x}^{\mathbf{e}} \mathbf{e}$ siècle $(70)$. La cheminée murale donne elle-même un indice de datation : en ville et dans un palais, elle trouve parfaitement sa place dans le courant du xmile siècle (71).

Ponctuellement encore, cette datation est confirmée par le démontage du mur 96 et la fouille de sa tranchée de fondation. La même céramique à pâte claire glaçurée est attestée - - avec un seul tesson également; un denier de Melgueil à légende dégénérée y a été également recueilli ( $\left.n^{0} 37\right)$. La céramique commune à pâte claire est aussi attestée dans la tranchée de fondation du mur 91 .

Tous ces indices mous renseignent sur la date de la modification intervenue par rapport au plan primitif mis en place au temps de la cour romane. On constate dorénavant la présence de céramique commune glaçurée qui était absente du comblement des silos. C'est donc vers le milieu du xnI ${ }^{\mathbf{e}}$ siècle que s'opèrent dans l'aile sud des modifications substantielles, touchant en particulier l'extrémité orientale. 


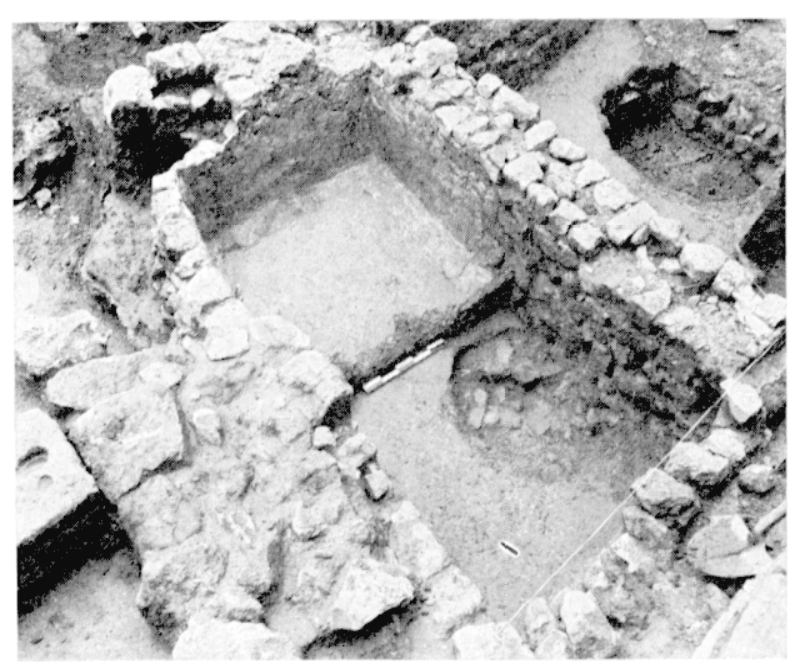

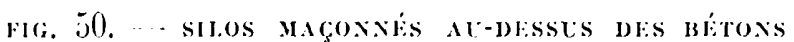

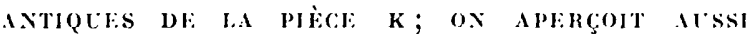
I. FMBOUCHCRE DE DETX SIIOS ANTÉRIETRS

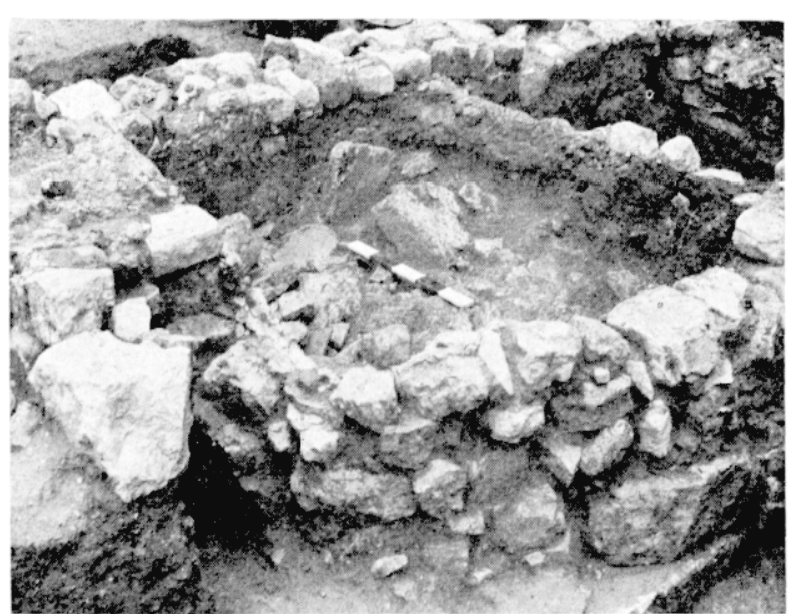

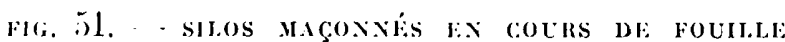

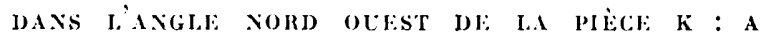

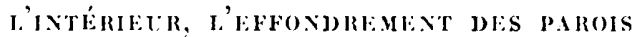

\section{3i IAE: DEVENIR DE: I.'AIHE SUD.}

Ise cadre architectural hérité de leépoque romane et du commencement de l'époque gothique était destiné a demeurer, dans ses grandes lignes, jusque vers la fin du xwe siècle, avec des modifications de détail (fig. 49). Parmi elles, on insistera d'abord sur le cloisonnement du passage dont l'extrémité nord est isolée par la construction du mur 90. Celui-ci vient s'appuyer sur la tête du nur 72. Mais le passagre subsiste encore vers le sud. Son usage semble cette fois limité à desservir ces petites pièces des communs. Son issue, jusque-là étroite au sud, est élargie par la construction du mur 99 qui coupe l'angle ancien formé au point de rencontre des portiques du cardo et du decumanus. Puis le mur 98, au-dessus des silos déjà comblés, vint s'appliquer sur le mur 90 . Il fut fondé au niveau des bétons antiques encore en usage dans l'espace VIb. Sa conception achève de donner une orientation oblique au passage. Cette modification progressive laisse penser que le débouché se faisait vers un axe créé au ILoyen Ige, qui allait devenir l'actuelle rue des Ayguesiers. Celle-ci n'a jamais été barrée par le palais comme on l'a toujours écrit ; elle en était l’issue normale à partir des communs.

Sauf dans l'espace VI b, un nivellement général s'est peu à peu opéré. Le cloisonnement du passage fut suivi d'une recharge générale des sols qui plaça toutes ces petites pièces au même niveau d'occupation situé à la cote moyenne 204 (couches 3 b et 3 c, fig. 41). Le niveau était approximativement celui auquel se trouvait déjà la cheminée à la naissance de l'aile orientale.

L'espace VI b doit iaire l'objet de remarques particulières. Plus longtemps que les autres pièces adjacentes, il servit à la conservation des grains selon les méthodes traditionnelles d'ensilage. Le catalogue des céramiques découvertes dans deux des silos de cet espace fait apparaître la présence de poteries à pâte claire vernissée, ce qui ne se retrouve qu'une seule fois dans un autre silo plus au sud, à l'extérieur des bâtiments. Ces deux silos creusés

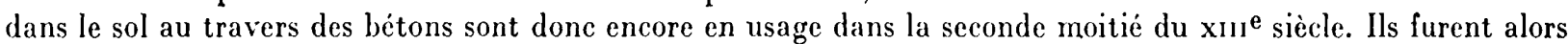
remplacés par des silos construits à même le sol antique, avec des parois maçonnées enduites du même mortier gris qui recouvrit d'ailleurs, au même moment, tous les murs de l'espace VI b. Le mur principal de cet aménagement, le mur 101, fut ancré à sa base dans le mur 73. On imagine que la raison en fut la stabilité recherchée contre la poussíe latérale qui s'exerçait une fois les silos remplis. Ceux-ci étaient séparés par la cloison 102 (fig. 50 et 51 ). Engagé sous la fontaine de Charles de Vintimille, l'espace VI b n'a pas été fouillé exhaustivement. Mais on sait que, dans le courant $d u$ xı $v^{e}$ siècle, les serviteurs de l'archevêque marchaient encore sur le béton antique adjacent aux silos. Les deux alvéoles bâtis, substitués dans le courant du xıv siècle aux silos creusés, font penser, comme la cheminée, aux exemples ruraux du village de Rougiers. Ils invitent à considérer des techniques finalement peu différentes à la ville et à la campagne dans des contextes résidentiels (72).

L'encaissement du sol de l'espace VI b par rapport au niveau des pièces adjacentes et par rapport au passage fut bientôt supprimé par la destruction des deux silos maçonnés et le comblement qui suivit (fig. 41). Cette égalisation fut marquée par la mise en place d'un sol argileux de $0 \mathrm{~m} 10$ à $0 \mathrm{~m} 20$ d'épaisseur contenant un matériel caractéristique du courant $\mathrm{du} \mathrm{XIV}^{\mathrm{e}}$ siècle, dans lequel apparaissent les productions de céramique à pâte rouge vernissée, de rares importations soit de Catalogne, décorées au cuivre et au manganèse, soit de la région de Valence au bleu de cobalt et au lustre métallique, et quelques productions régionales au décor vert et brun (73). 


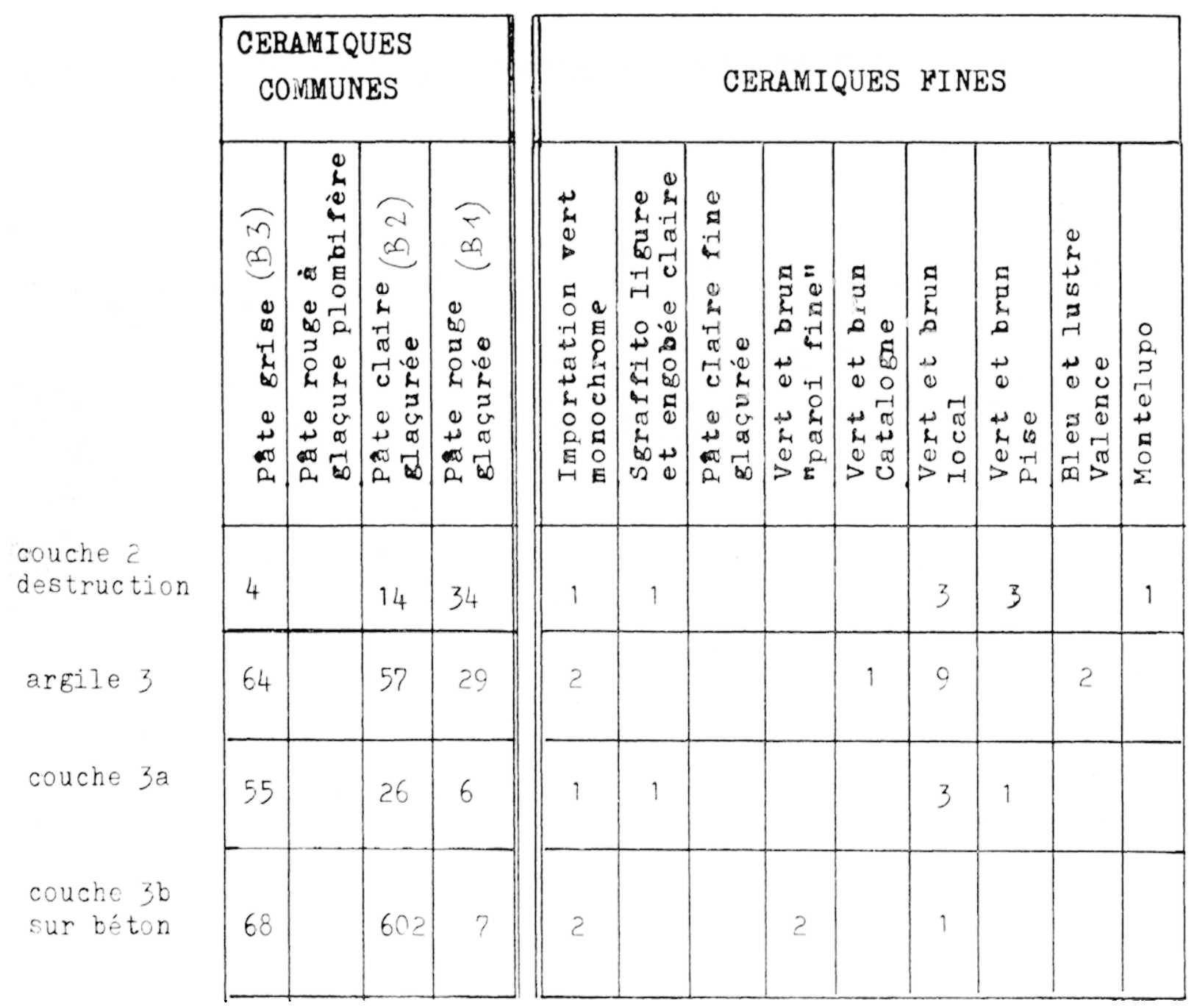

TabLEAU XII. Répartition des céramiques dans les couches de l'espace VI b.

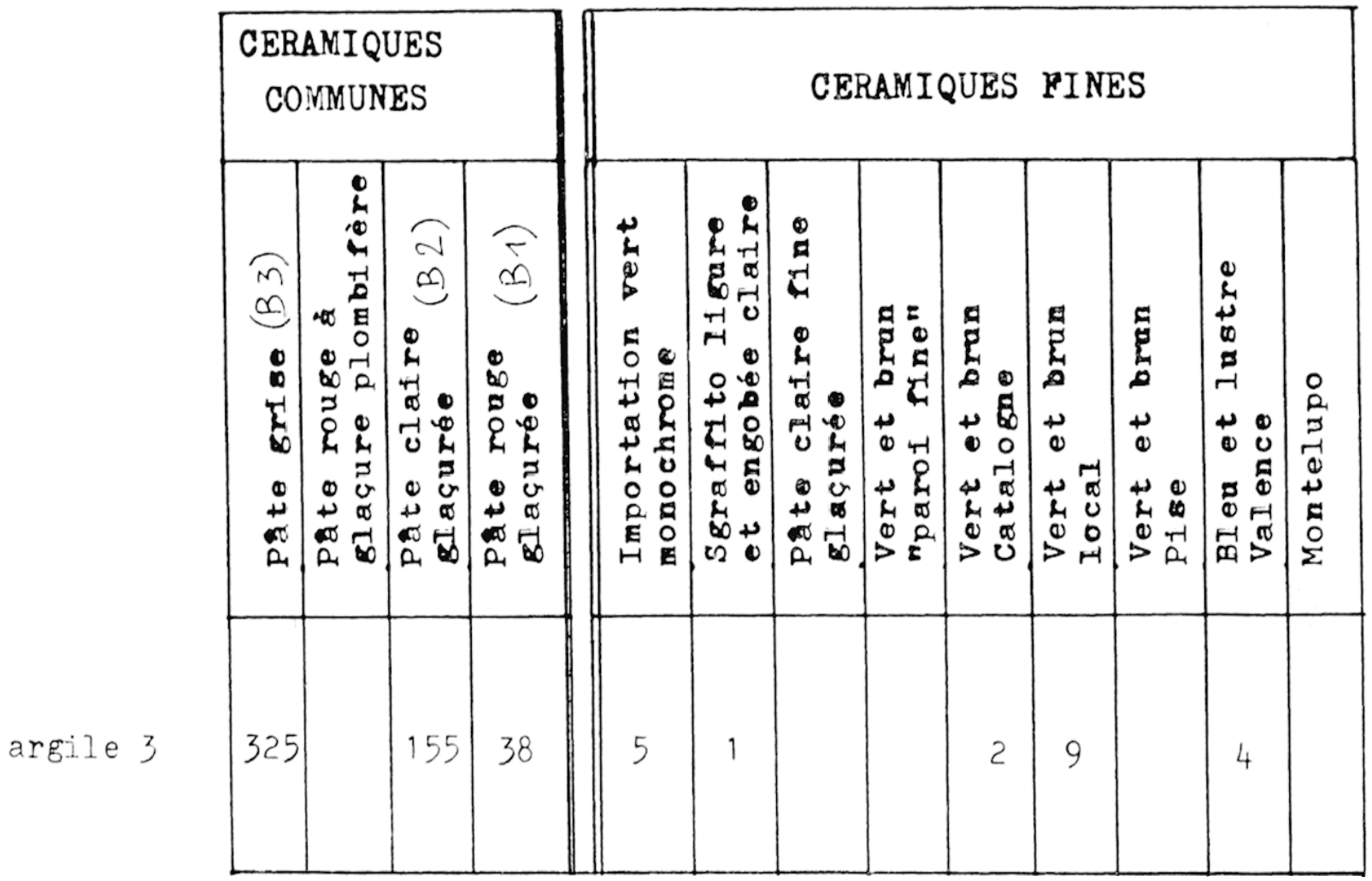

TABLfau XIII. Répartition des céramiques de l'argile 3 dans les espaces $\mathrm{Va}, \mathrm{Vb}, \mathrm{VI}$ a, VI b et VII. 
Les indices monétaires ne sont pas très abondants, et les renseignements qüils apportent domnent des témoignages moins précis. La monnaie f́1 évoque la fin du xun èècle. Lin jeton de hanquiers lombards, identique

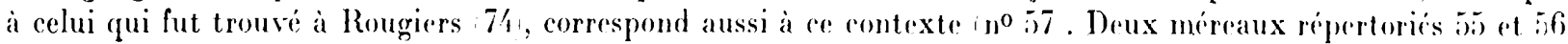
n'apportent rien de plus. On remarquera seulement combien leur décor cqui combline traits et besants sapparente a celui de la céramique dite à fond marqué découverte dans la vallée du Rhòne. à une date il est rrai, anterieure a celle à laquelle ces plombs furent vraisemblablement émis 75 .

La rapidite nécessaire de la fouille des niveaux de surface, limportance aussi des terrassements de toutes sortes comme les canalisations nombreuses de l'épocque moderne ou les plantations d'arheres ne favorivèrent pas toujours la pereeption des niveaux d'utilisation ultime ou d'abandon. Queldques indications peruvent etre cependant suggéries pour finir.

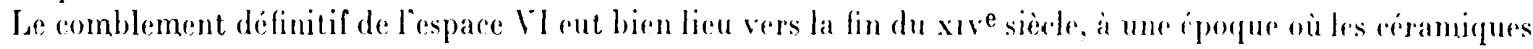
pisanes apparaissent dans les inventaires, dans les mêmes ensembles que les críramiques à décor au bleu de coblalt et au "lustre métallicque " en provenance de la région de Valence, mélés avee la production locale décorée au vert de cuivre et au brun de manganèse. Céest la même association que l'on trouvait dans une couche de terre grise et fine recourant larase superieure du mur 91 après sa destruction. Iussi peut-on penser que vers la fin du xire siècle, dans son état médiéval. l'aile sud du palais avait véeu.

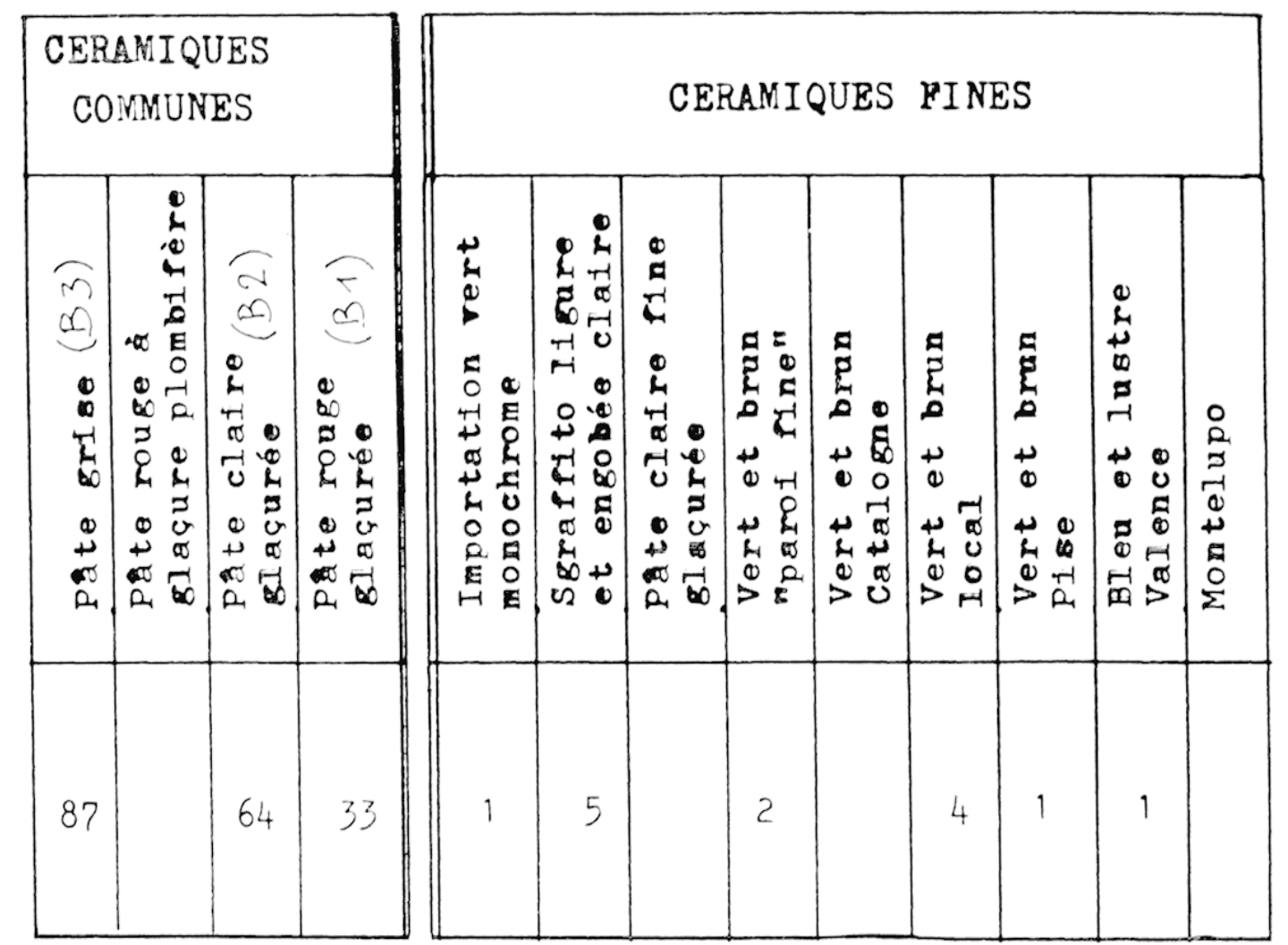

T'anteac XIV. Répartition des ciramiques dans les couches dabandon au-dessus des murs 91 , 97 at 98 lispaces Yll sud et VIII b sud.

b) Liaula de la seconde moitié du XIII e siècle.

\section{1) Description.}

Au nord de la cour, l'alignement roman d'origine antique fut abandomné apres la date d'apparition des premières céramiques à pâte claire vernissée. C'est ce dont temoigne la fouille de la tranchée de récupération des matériaux du mur ${ }^{4} 3$. In drain prit place alors, à l'est, au fond de celte tranchée. La fouille de la zone IX montre également une reprise de l'ancien angle nord-est de la cour romane. Le mur $\$ 3$, bàti dabord dans le prolongement du mur antique, fit dorenavant retour avee le mur de la façade orientale de la cour. On fit, comme réparation, une sorte de chaîne d'angle (fig. 45 ).

Ces deux indices laissent penser que le mur nord de la cour - ou l'aile nord - a alors disparı, sans doute pour être reporté plus loin. In espace sípare dorenavant l'aile orientale et l'aile septentrionale. Il y a donc eu agrandissement de la cour romane. A quelle date, et pour quelles dispositions nouvelles? 


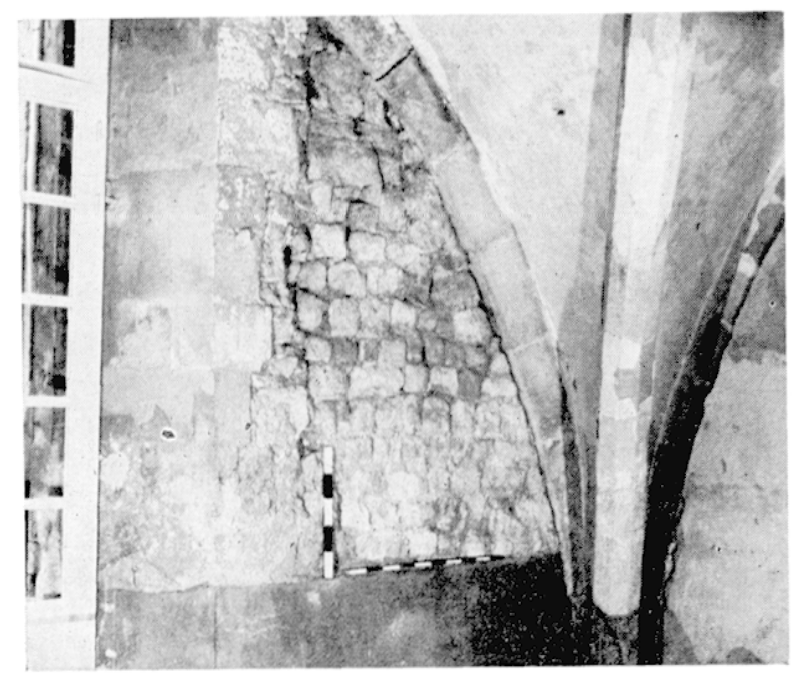

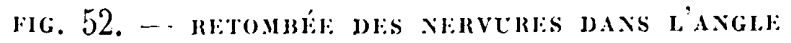

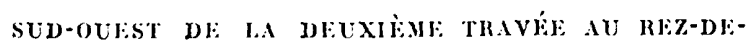
ChaUsSíte DE l.' "ALTA " A GAUCHE, L'UNE DES BAIES OCVERTLS PAR I'ARCHEVk̂QUE DANIEI. DE $\operatorname{cosiaC}$ A IA FIN DU XVile SI ÈcL:

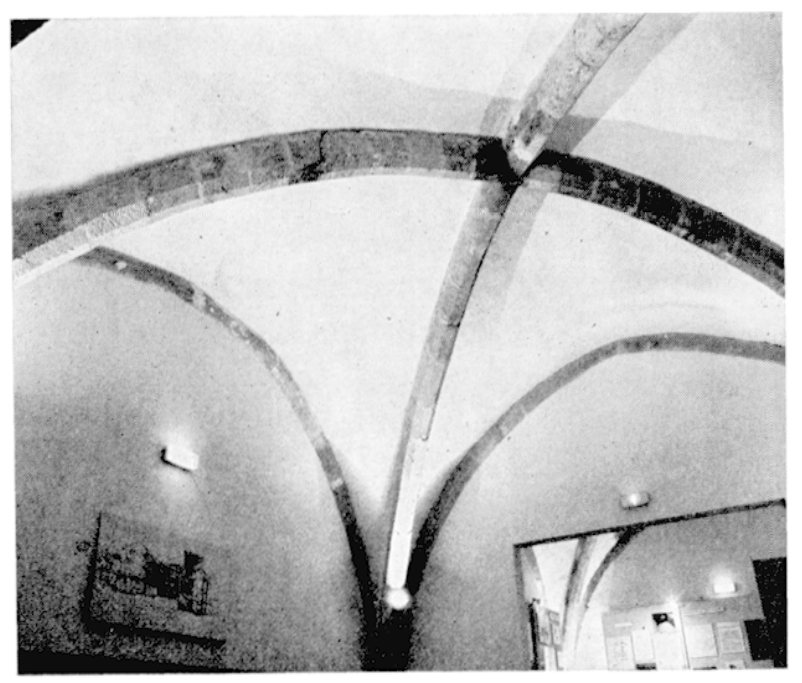

FIG. 53. … vô̂tr:Ml:NT

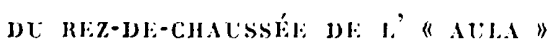

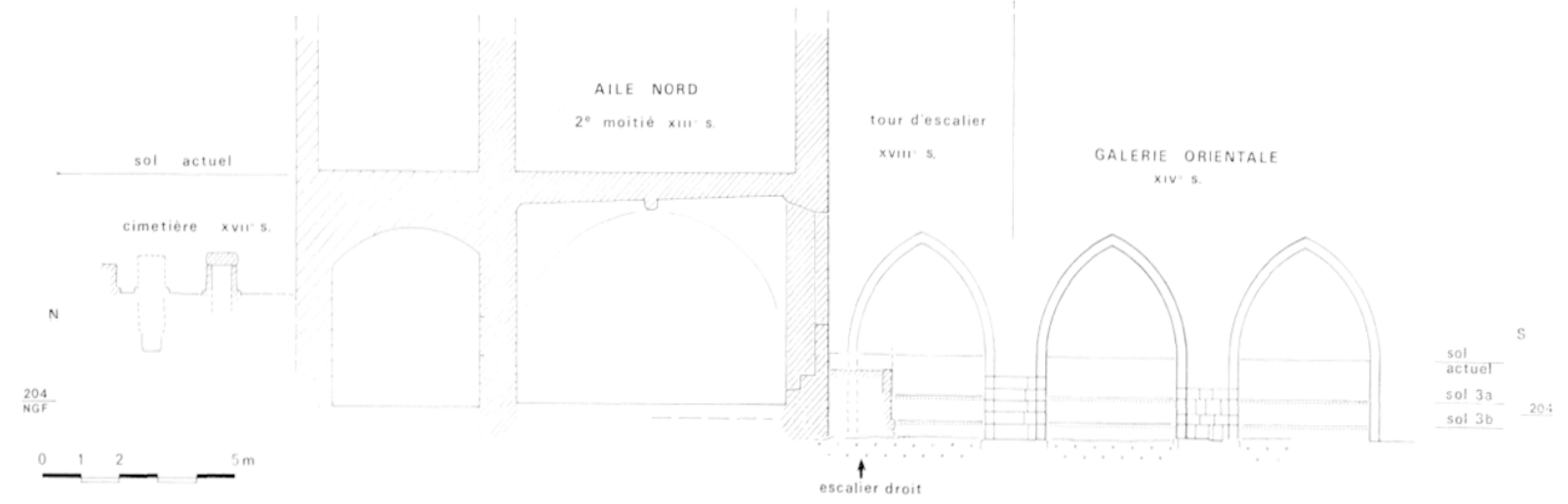

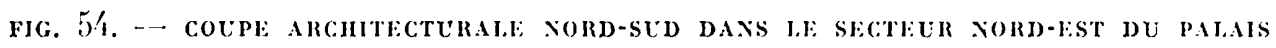

La fouille de ces parties a été incomplète à l'extérieur des bâtiments actuels, et exclue à l'intérieur où une chape de béton a maintenant recouvert les sols malonés, préservant ainsi les données pour le futur...

Je rez-de-chaussée de l'aile nord du palais actuel n'a attiré jusqu'ici que peu de commentaires. Sale, encombré, l'accès en était peu attirant avant les restaurations du printemps 1985. Le sol moderne de cet espace est situé en contrebas de la cour. C'était déjà la situation au xvir ${ }^{\mathrm{e}}$ siècle, alors qu'une partie servait à l'archevêque de salle à manger (76). Il s'agit en réalité d'un vaste volume, complant encore cinq travées voûtées sur croisées d'ogives. Des cloisons ont été montées à l'aplomb des doubleaux. Lne sixième travée se restitue sans difficulté vers l'est : son mur sud a été reconnu il y a pen de temps sous le pavillon d'escalier attribué à l'archevêque Daniel de Cosnac, et même la retombée des arcs a été portíe sur le plan du palais dressé en 1973 par les géomètres du cabinet Arragon (fig. 76). Cette dernière travée est adjacente à l'actuelle rue Pierre-ct-Marie-Curie, dont elle est séparée par un mur très épais. Il faut restituer là un superbe volume de 45 mètres de long, 7 mètres de large et une hauteur sous clef de $\ell_{4} \mathrm{~m}_{50}$ à partir du sol actuel. Jes murs latéraux ont une épaisseur moyenne de $1 \mathrm{~m} 10$. Ils sont bâtis en mollons très régulièrement assisés, visibles dans les lacunes de l'enduit jusqu'à la base du premier étage. $\Lambda$ l'extérieur, la fouille, puis les terrassements n'ont rien révélé : le mur est lisse (fig. 55)).

A l'intérieur, le volume est conçu pour donner une impression de simplicité et de légereté. Le rapport des arcs formerets et du mur montre que les voûtes sont contemporaines de l'élévation Un décroûtage a été réalisé dans les murs sud de la seconde travéc pour le vérifier (fig. 52). Le profil des nervures est austère. Le parti d'abattre seulement les arêtes releve des principes simples du découpage en facettes cher aux architectes du gothique méridional (fig. 53). Elles sont ramenées jusclu’à terre par trois supports indépendants, mais sans l'intermédiaire de chapiteaux. Cette absence est d'autant plus remarquable 


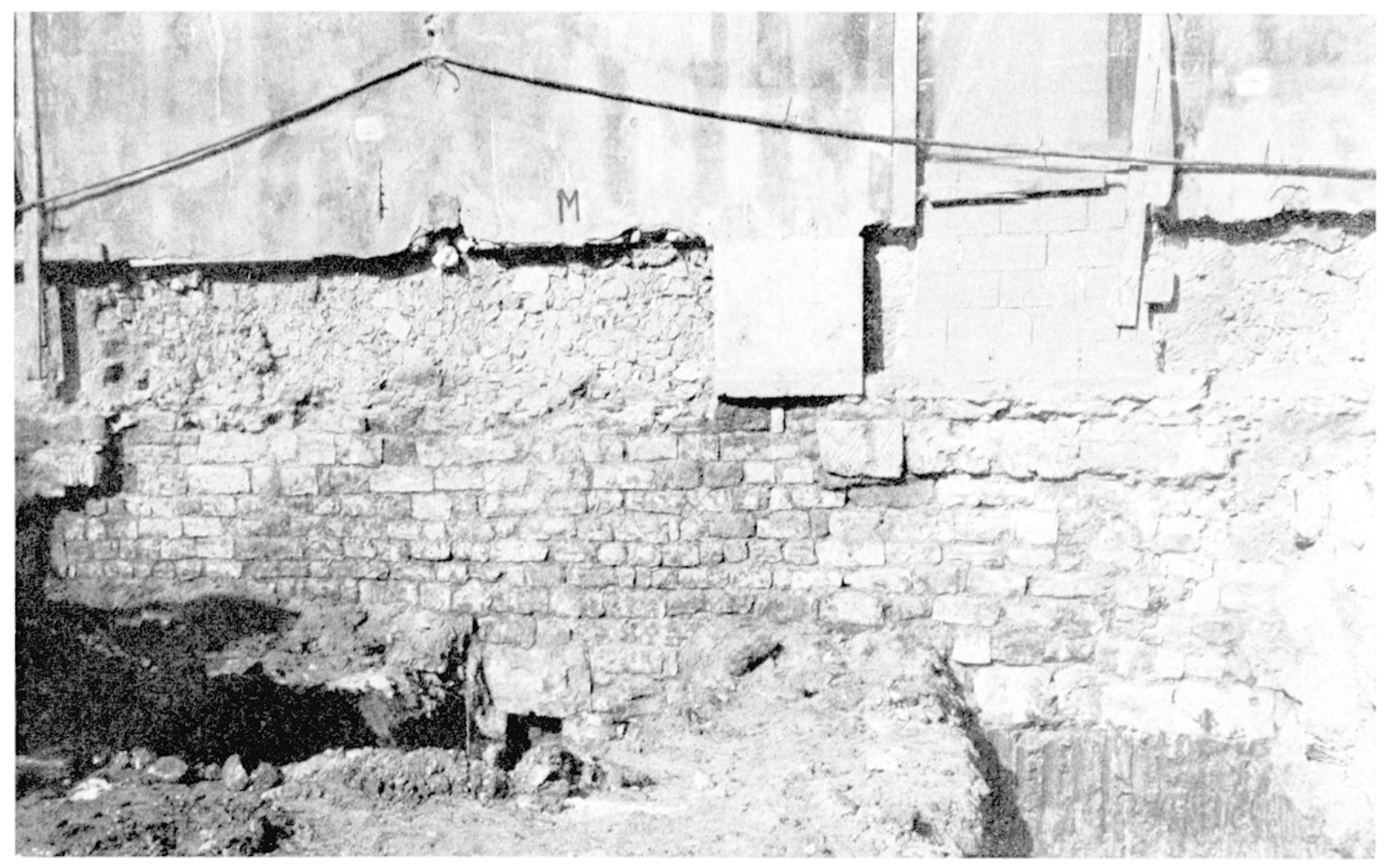

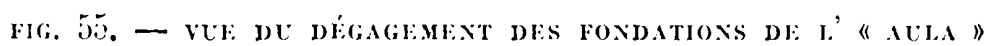

qu'elle a donné lieu à une certaine maladresse. Le support proprement dit est formé de trois assises; au-dessus, le départ des arcs se marque par une brisure nette, et la continuité est imparfaite. Les clefs, quant à elles, ne sont pas décorćes, dans cette salle dont l'aspect dépend des seules lignes architecturales en l'absence de tout décor sculptí.

Vers le sud, elle est éclairée par quatre baies percées dans chacune des travées donnant sur la cour. En 1708, ces ouvertures avaient été faites "depuis peu ". Elles doivent appartenir aux travaux elíectués sous l'impulsion de l'archevếque Daniel de Cosnac, tout comme le grand escalier dont il a été question plus haut, et qui occupe la sixième travée. Larges de 2 mètres au maximum de leur ébrasement intérieur, montant jusqu'à l'intrados du formeret, elles ont fait disparaître toute trace de baies antéricures, à l'exception d'une seule qui toutefois n'est sans doute pas d'origine (fig. 71). Aucune ouverture n'est visible non plus dans l'état actuel du mur nord, que ce soit de l'intérieur ou au revers du mur. Avant 166't, on sait qu'il n'était encore percé d'aucune porte.

Le sol maloné semblait être en place depuis le xvmi ${ }^{\mathrm{e}}$ siècle. Il était à la cote $20^{\prime}$, et c'était déjà la recharge de sols antéricurs. Les bases des supports des arcs sont ainsi ennoyées. C'est donc en fonction d'une cote situce approximativement vers 203,70 qu'il faut raisonner et établir des comparaisons avec le nivellement général (fig. 5'́l). Cé niveau n'est qu'à quelques centimètres au-dessus de celui des sols antiques dans les espaces $\Lambda 1$ et $\Lambda 2$ silués tout près de là, au sud. L'étage que portait cette salle, au début du xvir ${ }^{e}$ siècle, était "voûté à croisillon ". A l'extrémité orientale, adjacente aux dernières travées, se trouvait la chapelle, dont le voûtement était alors en mauvais état.

Deux sondages préalables aux terrassements ont été faits pour étudier les fondations. Celles-ci sont posées sur l'argile naturelle, à des cotes variables selon les campagnes de construction, à 203,30 à l'est, à 202,85 à l'ouest. L'assise la plus basse est faite de blocs de libage, grossièrement équarris, hauts de $0^{\mathrm{m}} 30$ à $0^{\mathrm{m}} 45$ environ. Sur eux sont posées, en retrait, une ou deux assises qui rachètent l'irrégularité, puis commence, avec un nouveau retrait, une élévation parfaitement régulière. Les fondations ne sont donc pas très puissantes. Mais l'épaisscur des murs, l'étroitesse relative des volumes intérieurs permettent évidemment d'imaginer un étage.

On remarque différentes campagnes de construction. De la première, il a déjà été question : elle est visible dans le mur nord de la première travée, et il est sûr qu'elle ne correspond pas au projet du monument que l'on a sous les yeux. La paroi est plus épaisse, atteignant $1^{\mathrm{m}} 30$; elle est surmontée par une corniche saillante, marquant vraisemblablement le départ du voûtement en berceau. $\Lambda$ l'est, dans la travée suivante, le mur est encore un peu plus épais que dans les autres travées.

Sur le mur nord, des interruptions affectent aussi la construction. La première se trouve à $11 \mathrm{~m} 50$ de l'angle nordouest de la cour actuelle. En plan, elle est soulignée par une sensible déviation de l'alignement. Sous la baie ouverte au $\mathrm{xvir}^{\mathrm{e}}$ siècle dans cette travée, la coupure est matérialisée par l'irrégularité des assises qui résulte d'un rattrapage des lits laissés en attente, et par une différence des mortiers. Une seconde interruption se lit à peu près dans l'axe de la travée suivante par une même irrégularité d'assises et une remontée du niveau des fondations par amenuisement des blocs de libage (fig. 55). Il s'agit là, comme on s'en rend compte, d'interruptions du chantier de courte durée qui n'affectent pas l'unité de 
l'ensemble. Leur aspect indique que la construction a progressé d'ouest en est en prenant appui au départ sur une élévation romane antéricure.

\section{2) Datation et ixterpkétatiox.}

Tout le monde s'accorde à reconnaître que cette partie est la plus ancienne subsistant dans le palais archiépiscopal. On la date volontiers du xve siècle, epoque de linstallation supposée des archevêques dans le Bourg Saint-Sauveur 77. Des préxistences sont parfois suspectées à cause du niveau très bas du sol par rapport à celui

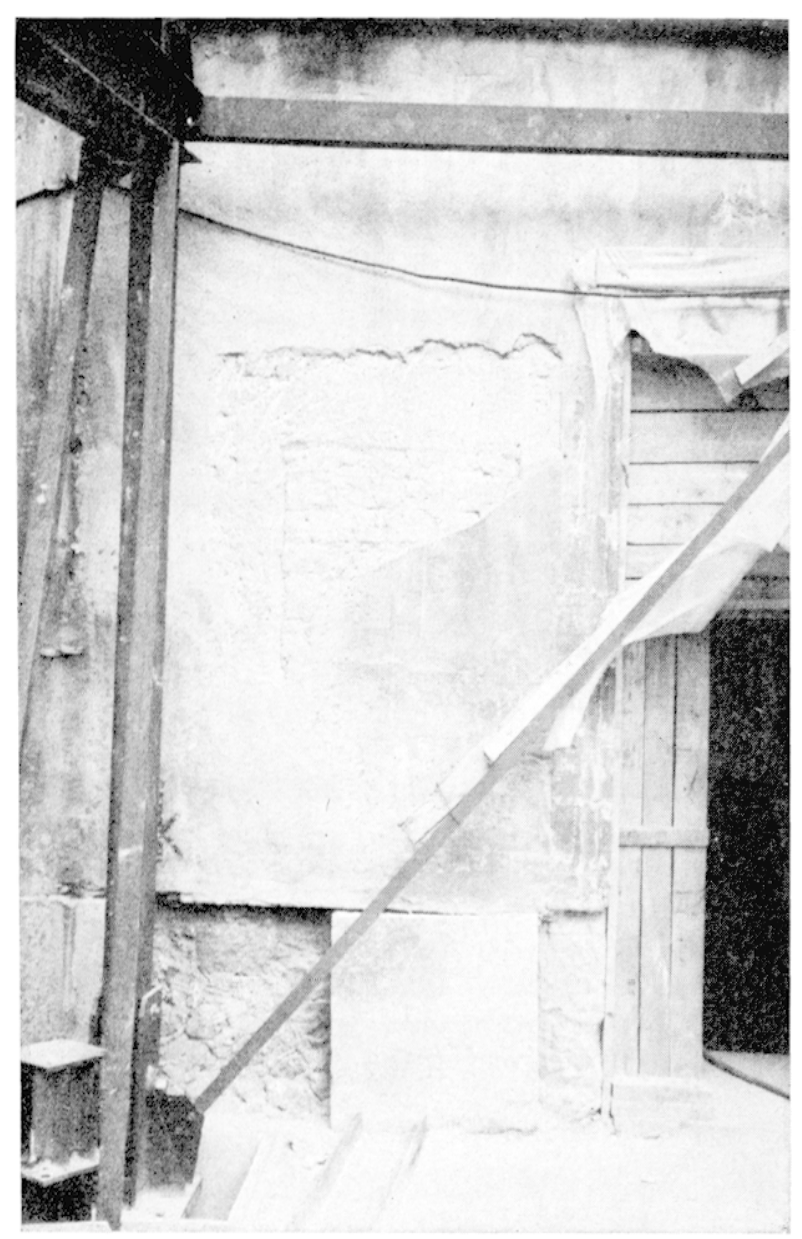

Fic, j6, - TRACE D'OUVERTURE DA.NS r.A TrOI-

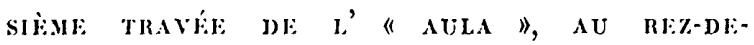
chatssí: Nais le sol 3 b, à la cote 203,81, ne contenait que des céramiques communes grises ou à pâte claire vernissée.

Linsi, le niveau intérieur est-il établi, dès l'origine, très peu au-dessus des sols antiques et à une cote qui, pour l'extéricur, correspond à des couches du courant du xum ${ }^{\mathrm{e}}$ siècle. On peut dire dès l'origine, car s'il y avait eu un recreusentent tardif, cela n'aurait pas manqué de faire apparaître soit la base des supports, soit leur fondation. Fit puis, bien que la distance soit relativement importante, de l'ordre de 40 mètres, rappelons que le sol originel du rez-de-chaussée de cette grande salle est encore à $1 \mathrm{~m} 30$ au-dessus du niveau du dallage actuel de la nef gothique de la cathedrale.

Il est enfin facile d'identifier l'édifice qui a donné naissance à l'actuelle aile nord du palais archiépiscopal avec un type de bâtiment courant de l'architecture résidentielle médiévale, tant laïque que religieuse. L'image répond exactement à celle de l'aula à deux étages, le niveau bas, susceptible de servir de communs, portant l'étage noble, la salle d'apparat de plain-pied avec une chapelle, qui était peut-être déjà située au nord de l'aula, de manière adjacente à ses travées orientales. D'une architecture très simple, l'étage bas paraît avoir été sans accès direct depuis l'extéricur. Vers le nord, on l'a vu, les premiers percements datent du xvir e siècle. Vers le sud, l'enlèvement des terres a fait apparaître le mur, sous la plinthe appliquée à la partie basse de la façade, plinthe qui prend appui 


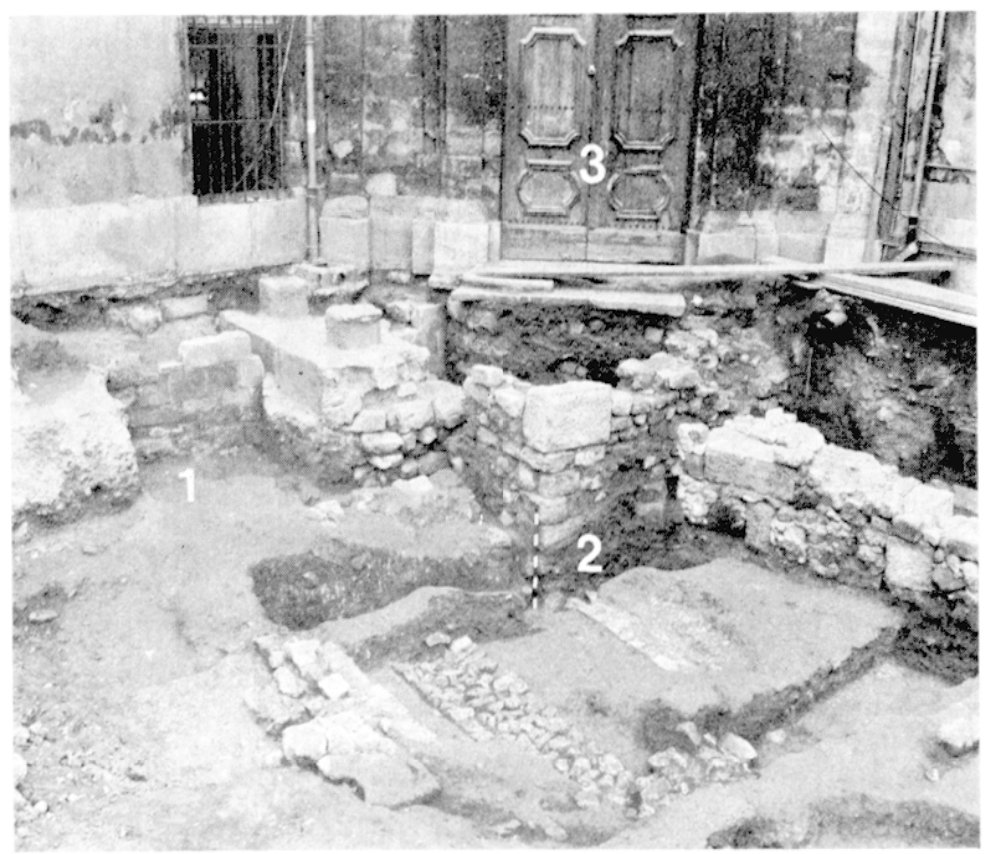

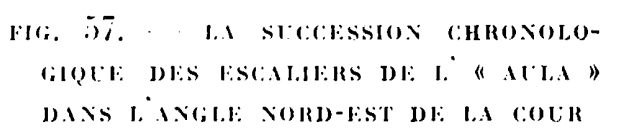

1) restes de l'escalier droit entre les plots en beton du theatre de Cassandre; 2 ) fondalions de la tour attribuéc à l'archevèque Pierre le Filleul $\left(\mathrm{xvi}^{\mathrm{e}}\right.$ siecle); 3 ) porte dans le pavillon d'angle attribué à l'architecte I,aurent Vallon (fin du $\times$ vil $^{\mathbf{e}}$ siècle).

sur la calade du xvme siècle. (1) jusqua à ce siveau, devé environ de $1 \mathrm{~m} 30$ au-dessus du sol intérieur d'origine, aucune trace de porte n'est visilhe fig. joj). Dans la troisième travée une ouverture avait été localisée naguère grâce à une lacune de l'enduit. Cat cilement avait paru susceptible d'appartenir à la bâtisse d'origine. Mais l'étude faite apres decroûtage partiel suggère autre chose fig. 56 : a qui avait été considéré dans de mauvaises conditions d'observation, comme les claveaux d'un are retaille, nest qu'une suite de quatre pierres appartenant a un linteau appareille surmontant une ouverture muré haute de $2 \mathrm{~m}_{4}$ (0), large de $1 \mathrm{~m}_{50} 0$ au moins, avec un appui situé à la cote 205,59. La largeur réelle ne peut pas être rétablie, car l'ouverture faite depuis par l'archevêque Daniel de Cosnac, dans cette travée comme dans les autres, a detruit le montant oriental. Cette baie, ou cette porte, s̈inscrit exactement dans la lunette de l'are formeret. Aucune autre ouverture comparable n'a pu être décelée, quand on a profité de l'enlèvement provisoire de quelques dalles de plinthe. Aussi le caractère exceptionnel qu'elle revêt, son emplacement décentré, l'usage du linteau appareillé, anachronique par rapport à la datation qu'il parâit possible de retrnir, font penser que dans l’état d'origine, le rez-de-chaussée nétait pas directement accessible de l'extérieur. Il pouvait être celairé par d'étroites fenêtres percées dans l'axe des travées; le parti pris de conserver celle de la troisième travéc peut daillenrs expliquer le decentrement de la porte qui fut percée après coup.

I'étude des indices stratigraphiques et de lapparence architecturale justifie la remise en cause des datations traditionnelles, plus fondées sur une interprétation parfois approximative des textes que sur l'analyse archéologique et architecturale. Il faut proposer d'identifier le rez-de-chaussée de l'aile nord actuelle avec la partie basse d'une aula qui doit remonter à l'activité de l'archevêque Guillaume Visdomini, placé sur le siège d'Aix à partir de 1257. J. Pourrière a montré que 'e grand personnage avait marqué son passage par une vaste construction désignée en 1268 comme hospitium, ou stare nosum en 1266. Au xive siècle, cette bâtisse apparaît sous le nom de domus de Crotis, ou hospitium de Crotis, soit la "résidence des voûtes ", et c'était le siège de la curie (79). C'est le rez-de-chaussée de cette réalisation de prestige que l'on a toujours sous les yeux. Son ampleur est digne du personnage qui l'a fait réaliser : il était membre de la famille des vicomtes de Plaisance, neveu du pape Grégoire X et légat du pontife, conseiller de Charles d'Anjou. Cette aula se rattache d'ailleurs à une série de grandes réalisations méridionales contemporaines, dues à un mécénat épiscopal dont le goût pour l'esthétique gothique nouvelle s'exprime dans de grands chantiers métropolitains, à Lix ou à Albi par exemple, bientôt à Narbonne. A ce titre, l'reuvre de Guillaume Visdomini peut s'apparenter, dans le profil des nervures et la simplicité architecturale à l'ceuvre de Bernard de Combret au Palais de la Berbie, dans la salle inférieure de l'Aile des Suffragants (80). Ce sont des réalisations grandioses qui sinspirent de la topographie des palais récemments achevés dans le Nord.

Comme cela a été noté, elles sont l'expression à la fois d'un goût nouveau, mais aussi de réalités politiques nouvelles dans le Midi, au même titre que les cathédrales qui sont entreprises simultanément et souvent concurremment dans cette période de richesse et de splendeur du pouvoir épiscopal (81). Aix apparaît comme un exemple révélateur avec la reconstruction de la nef nord de la cathédrale, provisoirement achevée vers 1285 sous l'épiscopat 


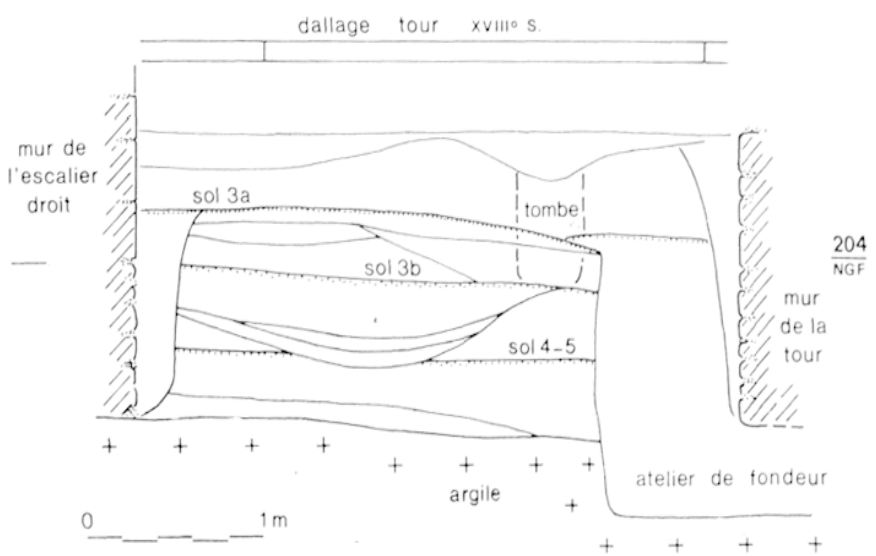

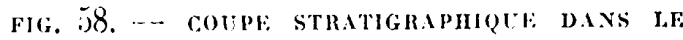

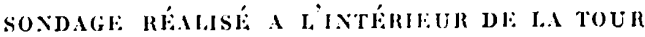
DI: PIIRRL: LE: FILITUU.

de Rostan de Noves (82). Lin effet, à Lix, comme souvent ailleurs, les travaux subirent le contrecoup du fléchissement des revenus, et les transformations traînèrent en longueur.

\section{3) L'iscatien Dhort.}

Si aucun accès direct au rez-de-chaussée ne peut-être altesté dans le schéma d'origine de cette aula, en revanche celle-ci a possédé, comme il est normal dans ce type d'architecture, une entrèe à l'élage accessible par l'internédiaire d'un escalier droit, parallile au mur et appuyé sur lui. Cette construction a été révélée par un sondage effectué dans l'angle nordest de la cour. La trouvaille a mis en évidence de manière impressionnante certaines continuités car cet escalier droit précède deux autres formes que prit ultéricurement la desserte des étages. Réalisé dans la même campagne que la transformation de l'aile orientale, il devait succéder lui-même à un premier escalier contemporain de la construction de l'aula, peut-être bâti en bois (fig. 49, 57 et 74 ).

Il était large de $1 \mathrm{~m} 70$ et on le montait vers l'est. L'entrée, au premier étage, devait se trouver au droit de la cinquième travée. Lee massif de fondation était posé sur l'argile naturelle, à la cote 203,50. line assise de mœllons formait talon, puis suivaient deux assises identiques en retrait, soutenant l'écevation appareillíe a partir du niveau 20',15 (fig. 54 et 58). Cetle différence d'aspect marque sans doute, approximativement, le niveau du sol de la cour au moment de la construction. L'utilisation de pierres de taille distingue cet escalier de l'aula, réalisée plus modestement en moellons réguliers. Le parement de la rampe contenait un blocage supportant les marches. L'état de conservation était extrêmement médiocre : déja affectéce par les escaliers ultéricurs, la maçonnerie avait été détruite lors de l'aménagement du théêtre dit de Cassandre par la mise en place de plots de béton destinés à supporter les gradins. Sur le massif ainsi endommagé, une seule marche était conservée, usée par le passage. Fille ćtait large de $0^{\mathrm{m}} 28$ avec une contremarche haute de $0^{\mathrm{m}} 18$. Siluée au niveau $20^{\prime}$ t, 13 , ce n'était pas la première marclıe de l'escalier. La trace de la précédente était visible au-dessous.

\section{c) La réfection de l'aile orientale : le promenoir.}

Nais déjà, lors de la construction de cet escalier, les bâtiments constituant l'ancienne aile orientale du palais roman avaient été détruits, et une nouvelle façade avait été créée vers l'est (fig. 49). L'aile ancienne, dont le réaménagement se marque, vers le milieu du xirre siècle, par la construction du mur 85 et l'installation d'une cheminée murale, ne fut ainsi qu'un temps contemporaine de l'aula. Lá nouvelle façade adoptait le tracé qui est encore le sien à l'époque actuelle, tracé qui rompt avec le respect des lignes directrices anciennes issues du plan de l'insula pour s'adapter à d'autres contraintes topographiques, sans doute à celle du rempart tout proche, ou même adjacent. Même si, dans son état antérieur, l'aile orientale avait pu ne pas être attenante à la muraille, et conserver ainsi un espace de circulation, il est probable que celui-ci avait déjà été interrompu par la construction de la sixième travée de l'aile nord. lans le nouvel agrandissement de la cour issue de celle de l'insula, tout passage entre l'enceinte et le palais devait être condamné.

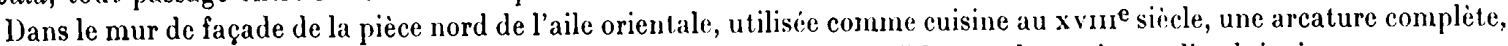
en arc brisé, était visible (fig. 59). La naissance d'une seconde était perceptible vers le nord sous l'enduit, interrompue par la masse oblique du pavillon d'escalier élevé vers 1690 dans l'angle nord-est du palais. U'u troisième arc, perpendiculaire aux deux précédents, se voyail, dans le mur sud de la pièce. L'expertise faite à la mort de Daniel de Cosnac précise que dinns cette cuisine "le sol est pavé de caillou, le haut est voûté à croisillon avec des ogives ett formerets de pierre de taille ". l'ne troisième travée existait au sud : " de cette cuisine, disent les visiteurs, nous sommes entrés au garde manger où étaient autrefois les archives... le haut est une voûte, partie de laquelle menaçant ruine doil être refaite ". Cétte travée était encore sous voûte en 1729 à la mort de Charles de Vintimille; elle ne semble pas avoir été alors réparée. Le rapport de 1708 domne encore ce renseignement un peu sybillin : «Il nous a été assuré que tout autour et au fond il y avait des contre-murailles 


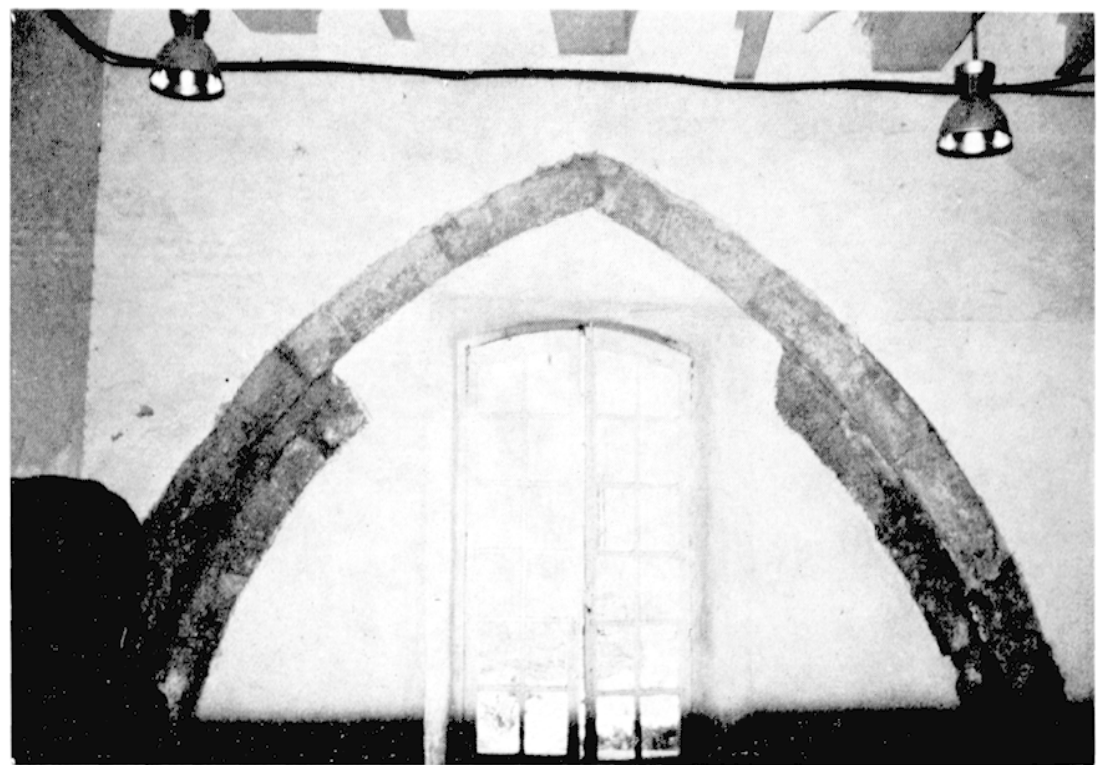

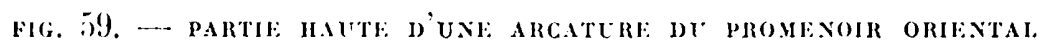

de pierre froide qui ont été tirées de l'ordre dudit feu seigneur archevêque ». Ces notations confirment d'ailleurs, en passant, le danger de toute surinterprétation d'indiés de continuté dans l'affectation précise des pièces, puisqu'ici, aux archives succède un garde-manger. Aussi la présence de la cheminée dans l'ancienne aile orientale ne peut-elle être mise en rapport avec la disposition postéricure et la présence de cuisines au $\mathrm{xvrrI}^{\mathrm{e}}$ siècle. Dans l'état ancien, il pouvait s'agir autant d'un chauffoir que d'une véritable cuisine.

Au commencement du $x_{v i n}{ }^{e}$ siècle, enfin, le même document atteste la présence de pièces, également voûtées, situées à l'étage, selon une disposition identique à celle de l'aile nord adjacente. les visiteurs notent qu'il y a là une galerie et un petit cabinet dont "le haut est voùté a croisillon avec une corniche autour, ayant des ornements d'architecture et quelques peintures aussi hien qu'aux ogives, formerets et compartiments... ". L'ossature d'un tres beau hâtiment, en trois travées, sans doute, apparaît, défiguré depuis, méconnaissable surtout à la suite de la destruction des voûtements.

Les parties hasses des arcatures reprises dans la façade ont été retrouvées lors de la consolidation récente des fondations. Autant d'ailleurs leur hase est apparue profonde par rapport au niveau de cour actuel, autant leur obturation, très haute, traduit une réfection de l'aile consicutive à un exhaussement général de l'espace central. Deux jambages, correspondant bien à trois arcatures, ont éte mis au jour, séparés par un intervalle de $33^{m / 10}$. Ils sont en pierre de taille, de mème aspect et de mème qualité que la rampe de l'escalier droit tout proche (fig. 5't et 60\%. Les denx ouvrages apparaissent ainsi contemporains. Reposant sur un massif de fondation implanté dans l'argile naturelle, ces supports sont appareilles a partir de la cote $203,3{ }^{\prime}$. Celle-ci est trés basse par rapport au niveau du sol d'origine restitué de l'aula. Iin fait, il est improbable que le sol correspondant ait été plus bas que la cote 203,80 , les murs rasés de l'ancienne aile orientale aflleurant tous à ce niveau, juste au-devant. Cette cote est celle du sommet de la première assise appareillée. Mais les trois assises inférieures semblent avoir été préservies trés löt par un remblai de terre montant jusqu'a la cote 204,20 . Aulant les parements sont nets audessous, avec des angles vifs, autant au-dessus ils portent des traces d'usure et de dégradatious. Cette cote correspond bien à celle à partir de laquelle l'escalier droit appuyé à la façarle nord a étí appareillé. Il est donc probable que les trois assises inférieures des arcatures ont été vite remblayées, le niveau d'occupation en terre, éclats de lauses, pierrailles et mortiers pulvérulents s'établissant vers la cote 20\%,20, environ $0^{\mathrm{m}} 50$ au-dessus du sol du rez-de-chaussie de l'aula, et sclon un rapport logique d'ensemble, puisque les comparaisons s'effectuent tres bien avec les cotes relevées dans l'aile sud. C'est à ce niveau que non loin de là, dans le sondage effectué à l'intérieur de la tour moderne de l'angle nord-est de la cour, se situe également un sol de consistance identique qui marque l'époque de la construction de l'escalier droit.

Le profil des supports est assez complexe (fig. 61). Comme dans le rez-de-chaussée de l'aula, il reproduit encore la composition des ares dans l'élévation, à l'exception toutefois de la retombée des ares diagonaux dont il faut imaginer l'amortissement dans les angles des travées. Vers l'extérieur, la pile présentait deux ressauts aux arêtes chanfreinées correspondant au double rouleau qu'on devine. Vers l'intérieur, plus richement animé, le triple rouleau de l'élévation conduit, en l'absence de fouille, à restituer trois ressauts auxquels s'ajoute un ressaut supplémentaire destiné à recevoir l'are doubleau. Comme dans l'aula, le principe de la continuité des supports et des arcs, aux arêtes simplement abattues, a été respecté.

La transformation suivait done les principes de l'architecture résidentielle de la fin du Moyen Age. Ine galerie largement ouverte sur la cour, voûtée, portait un étage d'appartements. lille semble avoir été limitée à trois travées alors que semblables espaces couverts, aux palais épiscopaux de Narbonne ou de Frejus, occupent une ou deux ailes. Ils peuvent aussi occuper les quatre côtés, au palais d'Avignon, mais parfois se limiter aussi à trois travées dans l'aile de la Grande Audience de Clément VI. Comme à Narbonne, la galerie occupe toute la profondeur du rez-de-chaussée du bâtiment (83). Complé- 


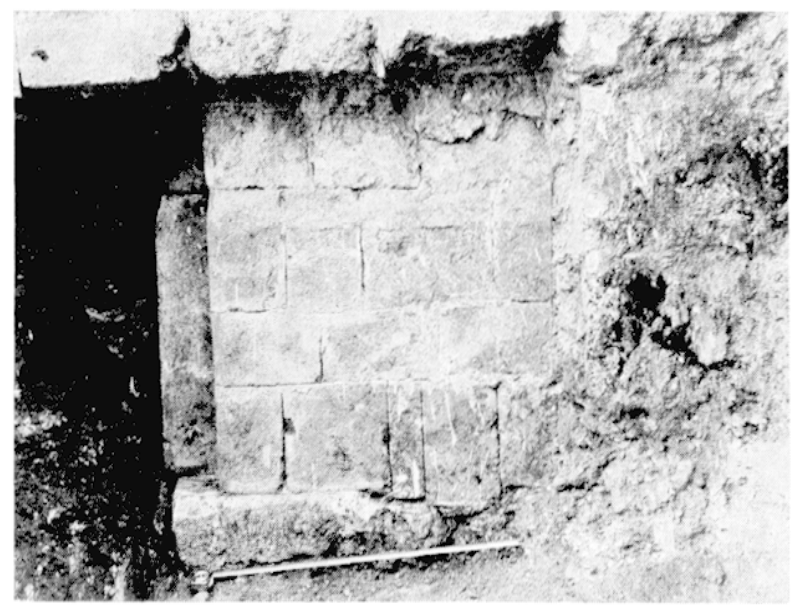

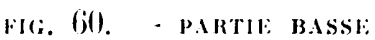

I) ITE: PILE DL PROMEXORR ORHENTAL

C. G. Lemaire.

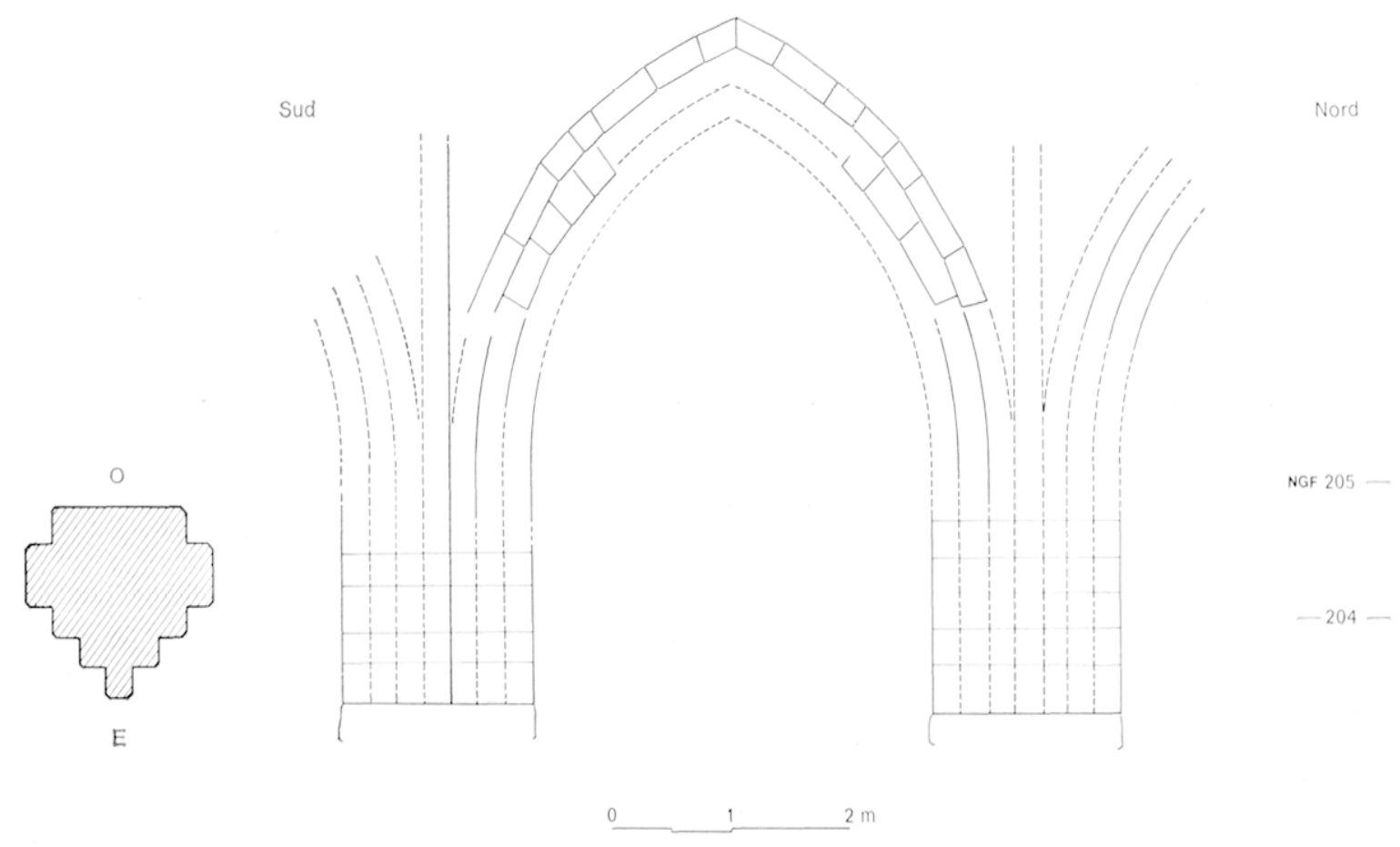

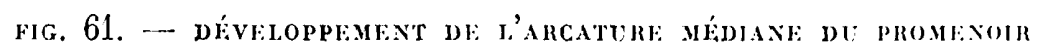

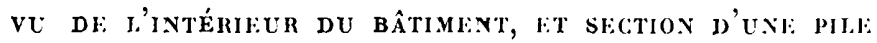

ment de l'aula, le promenoir et son étage donnaient au palais épiscopal d'Aix une allure monumentale, le mettant au goût du jour.

d) La cour.

Avant d'envisager plus précisément les problèmes de datation, il faut considérer l'espace central progressivernent agrandi et les traces de l'activité qui s'y déroule. Ce sera d'ailleurs l'occasion de recueillir de nouveaux indices chronologiques.

Par la démolition de l'aile orientale et la création du promenoir, la longueur de la cour, d'est en ouest, était portée à 30 mètres environ, pour une largeur de 20 mètres. Cette surface de 600 mètres carrés n'est vraie que si l'aile occidentale n'a été affectée par aucune transformation ou destruction. De cela il n'y a aucune trace. Mais la conservation de l'emplacement médiéval par l'aile actuelle semble bien indiquer la permanence de celte limite, toute cxpansion du palais vers l'ouest venant buter sur le quartier canonial, et en premier lieu sur la maison du prévôt el sur ses dripendances. Outre sa fonction normale de circulation et d'apparat, la cour assumait de temps à autre une fonction utilitaire. Trois ateliers de fonte de cloches ont été retrouvés. Outre l'intérêt archéologique que présentent ces cmplacements, ils apportent aussi des indices de datation, puisque deux d'entre eux furent établis sur les ruines des constructions romanes de l'aile orientale. Ia technique de fabrication, bien connue, n'a guère varié dans ses principes. C'est une activité qui est fréquemment constatée dans les 

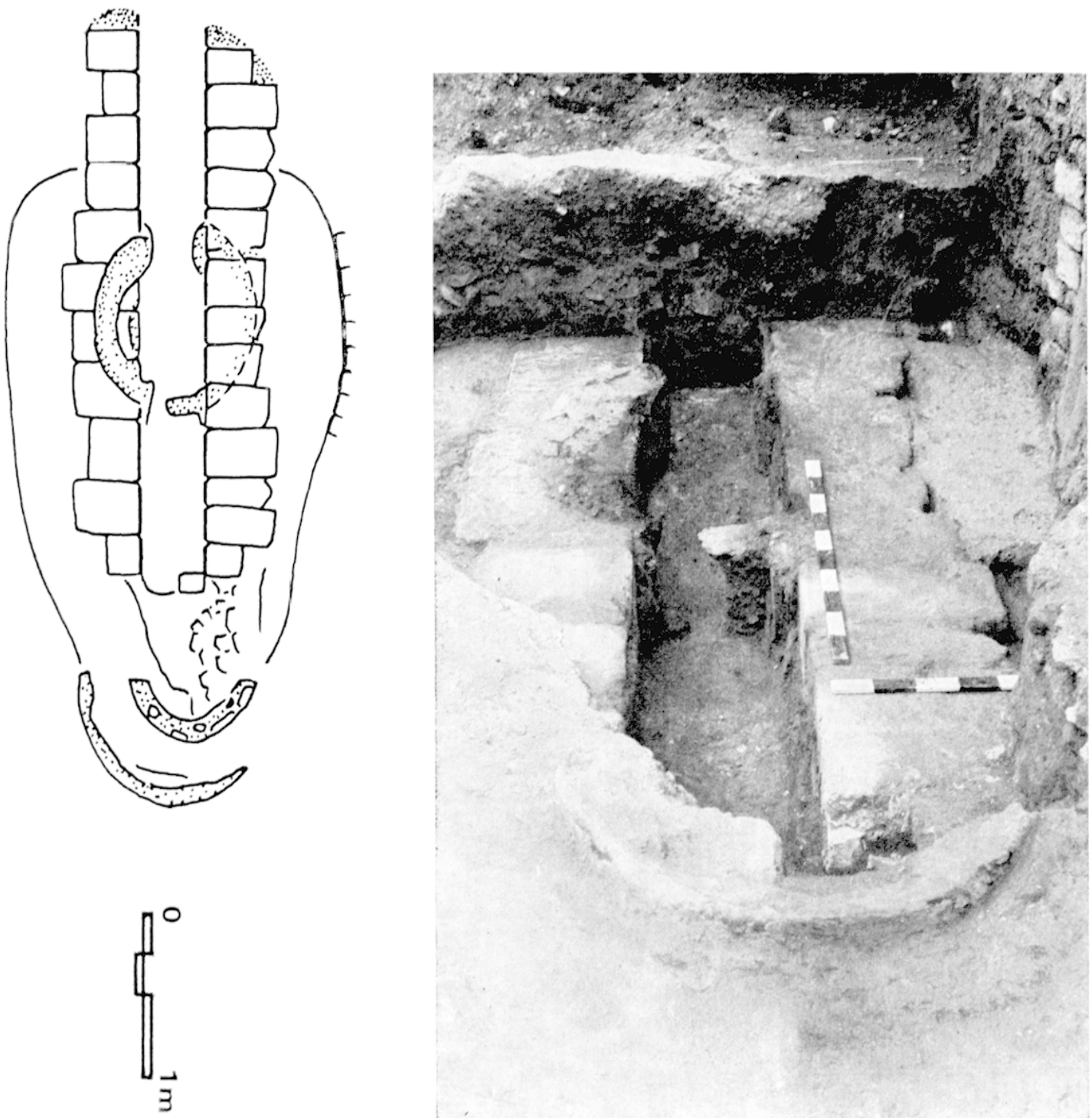

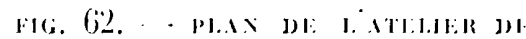

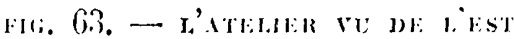

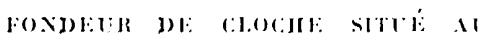

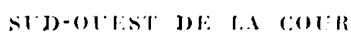

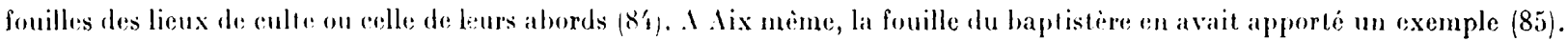
Les textrs illustrent a leur tour celte richesse par les fricfuentes mentions de cloches el de clochers qu'il n'apparait pas toujours facile de situer en mison de leur abondance. ("'est aussi la raison pour larquelle, en l'elat actuel do la documentation, toute attribution de ces cloches à un édifice particulier est sans doute illusoire (86j).

Le premier emplacement ful choisi juste devant la façade de l'aile mérielionale, dans la partie mediane de la cour

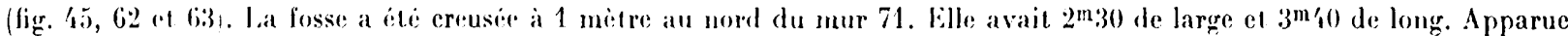

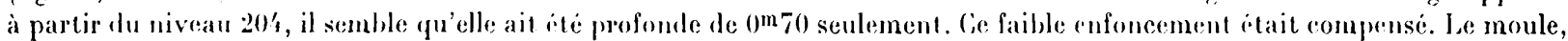
en effel, au moment de la fonte, avait pu cetre enterre sous un tumulus de terre contenu au sud par le mur du hatiment et au nord par un muret de pierre hâti a cet effet. Triss soigneusement devee avee des merllons de remploi antigues, sa paroi

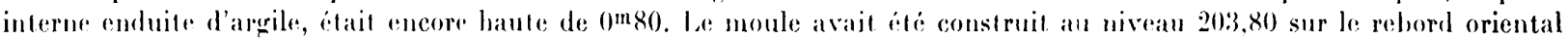
de la fosse. (11 sait que celui-ei est diabord forme du noyan, de forme troneonique (87). II en a cte retrouvé une partie de lat base, d'un diametre de 0 m90, faite de briques mal cuites. Celte construction ibail ensuite habille d argile mise en forme gràce a un gabarit tournaut sur un pieu d'axe vertical. sur ce noyau était appliquée la cire de la fausse cloche, puis à nouveau, l'argile du manteau. L'empreinte de cette derniere itait encore visible. Le diametre total du moule elait de 1 mato environ. l'ensemble itait ensuite séché. On le transportait done sur un foyer a l'endroil oi chait prévue plus tard la conlé du métal. Pour cela, un long alandier arait été construit qui tranchait les nurs antiques 11, 9, 1't et 16. I)isposé d'est en ouest, il était 


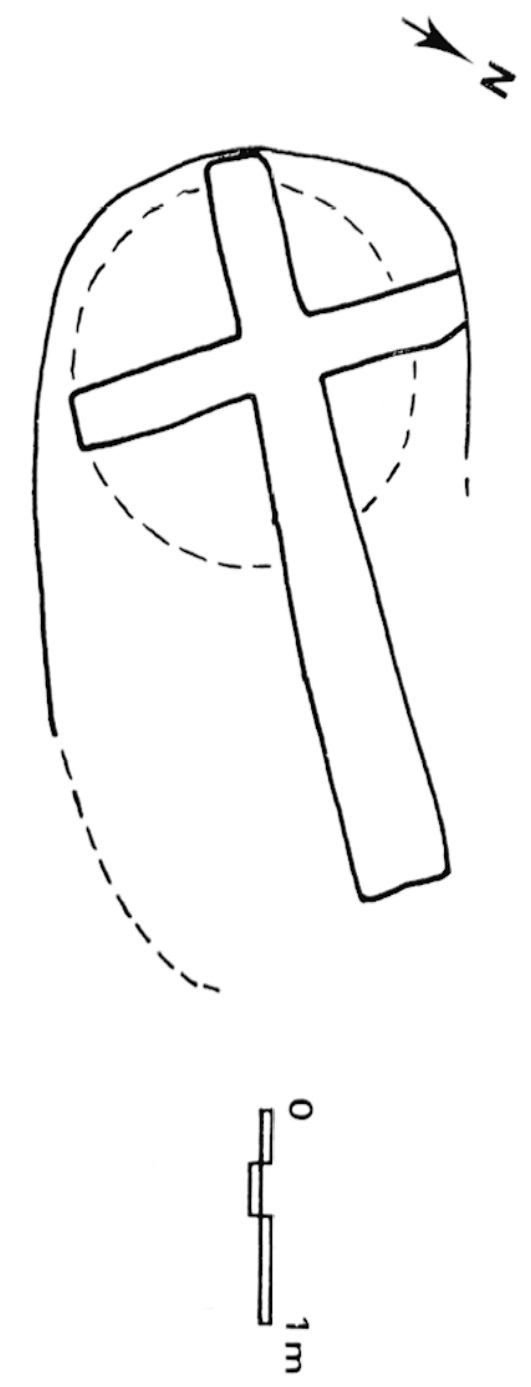

FIG. 64. - PLAN DE L'ATHLLHR DE FONDEUR SITUÉ AU NORD-EST DL: LA $\operatorname{COU} R$

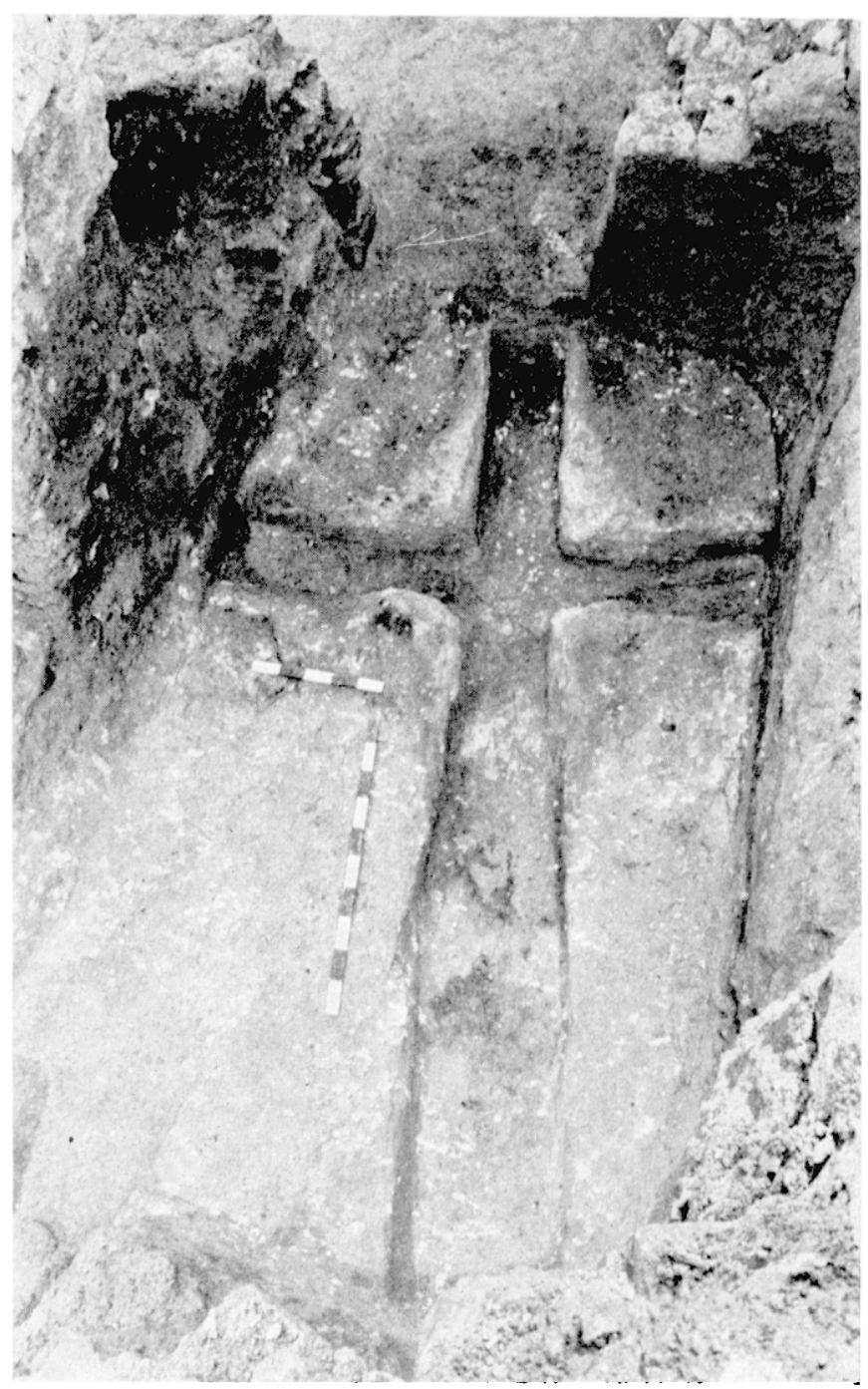

FIG. 6J. - L'ATELIER VU DE L'EST

conçu comme un couloir de $3^{\mathrm{m}} 50$ de long, $0^{\mathrm{m}} 40$ de large, $0^{\mathrm{m}} 30$ de profondeur. Le tiers des matériaux utilisés était constitué de blocs d'appareil roman avec les traces de taille et le module caractéristique. La face des pierres exposée au feu avait été enduite d'une pellicule d'argile, très épaissie à l'aplomb de l'endroit où devait venir reposer le moule, jusqu'à former une sorte d'arc diaphragme à l'ouest. Sans doute le but était-il de mieux conserver la chaleur. Lors de sa mise en place, le moule était basculé à l'intérieur de la fosse. Pour cette opération, il semble qu'on ait sapé le sol sous le moule préparé. C'est ce qui expliquerait la disparition d'une partie de la trace du noyau dont il ne restait qu'une forme en croissant en arrière d'une encoche dans le rebord de la fosse. Une fois le moule séché et la cire fondue, il était enterré comme on l'a vu, et le métal était coulé. Après refroidissement, l'ensemble était déterré, le moule cassé et la cloche nettoyée. Cette opératión explique la nature du comblement de la fosse, qui consiste en déchets de moule noircis par la chaleur, portant l'empreinte du métal, ainsi que des déchets de métal issus de coulées intempestives entre les parties basses du manteau et du noyau par exemple, puis du nettoyage de la cloche. Certains fragments de moule, parties correspondant aux colombettes, portaient la tracé d'un décor torsadé fait au doigt. Le bâti de l'alandier, très long par rapport au diamètre de la cloche et du moule, montrait d'autres traces de rubéfaction. On s'est donc demandé si, contrairement à ce qui se passe d'habitude, l'emplacement n'avait pas resservi. L'alandier était en effet plus long que la fosse faite à l'occasion de la fabrication de la cloche dont l'empreinte subsistait.

Le second four a étẻ retrouvé dans l'angle nord-est de la cour. La fosse est cette fois beaucoup plus profonde (fig. 58). Elle a été creusée depuis un niveau de cour, situé à la cote 204,20 , matérialisé par des déchets de construction, mortier pulvérulent et éclats de lause. Elle atteint une profondeur de 2 mètres à l'est, $2^{\mathrm{m}} 50$ à l'ouest. Comme la précédente, elle est large d'un peu moins de $2^{\mathrm{m}} 50$ et longue de 4 mètres. La cavité, grossièrement occidentéc aussi, a été faite sur l'emplacement du mur 62 , mur nord de l'ancienne aile orientale. L'alandier était cette fois de type un peu différent (fig. 6's et 65). D'abord, 

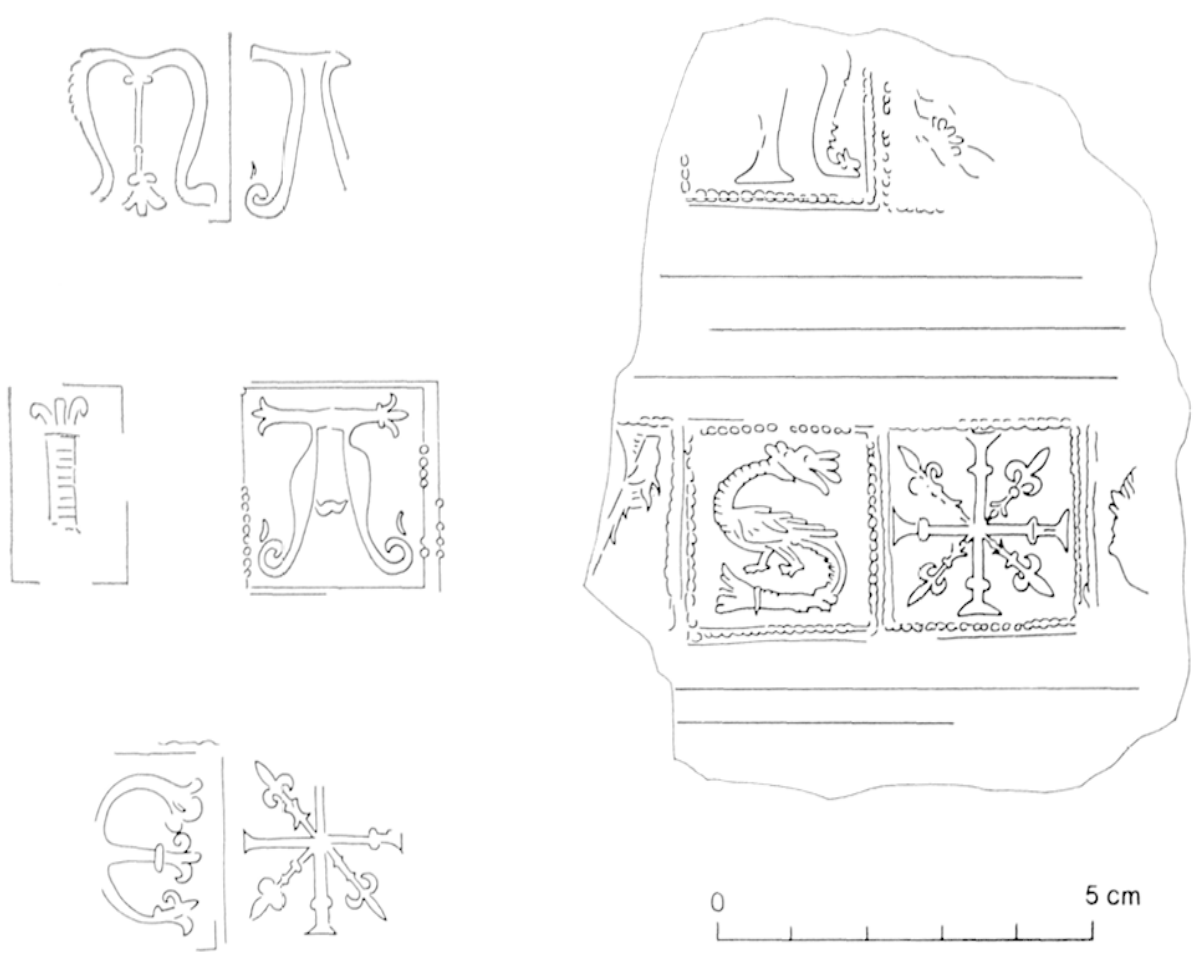

FIG. 66. -- FRAGMENTS DE DÉCOR DE CLOCHF:

DESSINÉS A PARTIR DE FRAGMENTS DF MOCLES

il était seulement façonné dans le substrat d'argile naturelle compacte, sans aucune pierre. Son plan était en forme de croix latine. L'axe principal a reçu des dimensions qui rappellent celles du premier four, $3^{\mathrm{m}} 50$ pour la longueur, $0^{\mathrm{m}} 50$ pour la largeur à l'est, $0^{\mathrm{m}} 30$ à l'ouest. L'axe transversa! avait $0^{\mathrm{m}} 25$ de large. Il n'y avait pas, dans les parages, trace de fabrication du moule. Son emplacement pour le séchage, à la croisée, avait laissé une auréole rubéfiée de $1 \mathrm{~m} 10$ de diamètre, ce qui correspond à une cloche sensiblement égale à celle qui a été fondue dans le four sud. Le comblement de la fosse était également composé de déchets de moule et de déchets de métal. Ici un certain nombre de fragments du manteau portaient des traces de décor, soit décor torsadé des colombettes imprimé par pressions successives du pouce en oblique dans l'argile fraîche, ou pour la panse lettres associées à des motifs rayonnants s'achevaut par une fleur de lys (fig. 66). Le comblement a été par la suite perturbé par la fondation de la tour d'angle.

Un troisième four se situe au sud-onest de celui-ci, et son implantation a encore eu lieu sur les restes enfouis des mêmes bâtiments romans (fig. 49). C'est, cette fois, l'ancien mur de façade, le mur 63, qui s'est trouvé coupé. I'image de la technique de fabrication est encore assez complète. Les limites du sondage correspondaient ici à un tracé imposé; aussi les rebords de la fosse ne sont-ils pas apparus clairement. L'emplacement de fabrication du moule était conservé (fig. 67 et 68 ). Il était marqué par l'assemblage en carrí de quatre grosses dalles de récupération, épaisses de $0^{\mathrm{m}} 10$ à $0^{\mathrm{m}} 15$, disposées à plat de manière à laisser entre elles un espace libre, au centre, pour l'enfoncement de l'axe portant le gabarit, ou planche à trousser. La surface ainsi soigneusement arrangée avait $1^{\mathrm{m}} 50$ d'est en ouest, et $1^{\mathrm{m}} 30$ du nord au sud, et se trouvait à la cote 203,05 . l.a fosse, au fond de laquelle avait été fait cet aménagement, paraît avoir été protégée au sud par un mur de pierre sèche, ayant une fondation asymétrique posée à 203,14 au nord, du côté de la fosse et à 203,84 au sud, du côté de la paroi de terre. Le sommet du muret, large de $0 \mathrm{~m} 50$, affleurait à $204,44 \mathrm{~m}$. Sur l'aire de sa fabrication, le moule avait laissé une trace d'argile fine. L'emplacement de séchage, ou de cuisson, et de fonte proprement dite était adjacent à 1 mètre à l'ouest. Une aire circulaire de $1 \mathrm{~m} 80$ de diamètre était délimitée par un murel à un seul parement intérieur, enduit d'argile, laissant un accès en glacis par l'est, comme dans les autres cas. Les assises conservies culminaient à 203,85. Ln alandier rectiligne de direction est-ouest avait été construit. Il était long de 2 mètres, large de $0^{\mathrm{m}} 40$, profond de $0^{\mathrm{m}} 35$, bâti en pierres de taille profondément rubéfiées, encore des remplois. L'une des pierres avait une arête chanfreinée, avec des faces layées à la gradine. Le rebord de cet alandier était à la cote 203,20. l'emplacement du moule était marqué par la rubéfaction, et aussi par l'empreinte circulaire de l'argile. Ia cloche avait des dimensions comparables a celles des deux autres, de l'ordre de 1 m10 de diametre. Certains fragments de moule issus du comblement porlaient egalement des décors imprimés, en particulier des lettres et des motifs rayonnants achevés par des fleurs de lys, jdentiques à ceux retrouvés dans la fosse du four voisin. la présence du même décor torsadé utilisé pour les colombettes n'est pas surprenante.

1 chaque fois, la fouille a seulement permis de retrouver les endroits où la cloche elle même avait été fondue, et dans deux cas, le lieu adjacent de fabrication du moule. Jamais il n'y a eu trace, à proximité immédiate, de lieu ou s'effectuait 

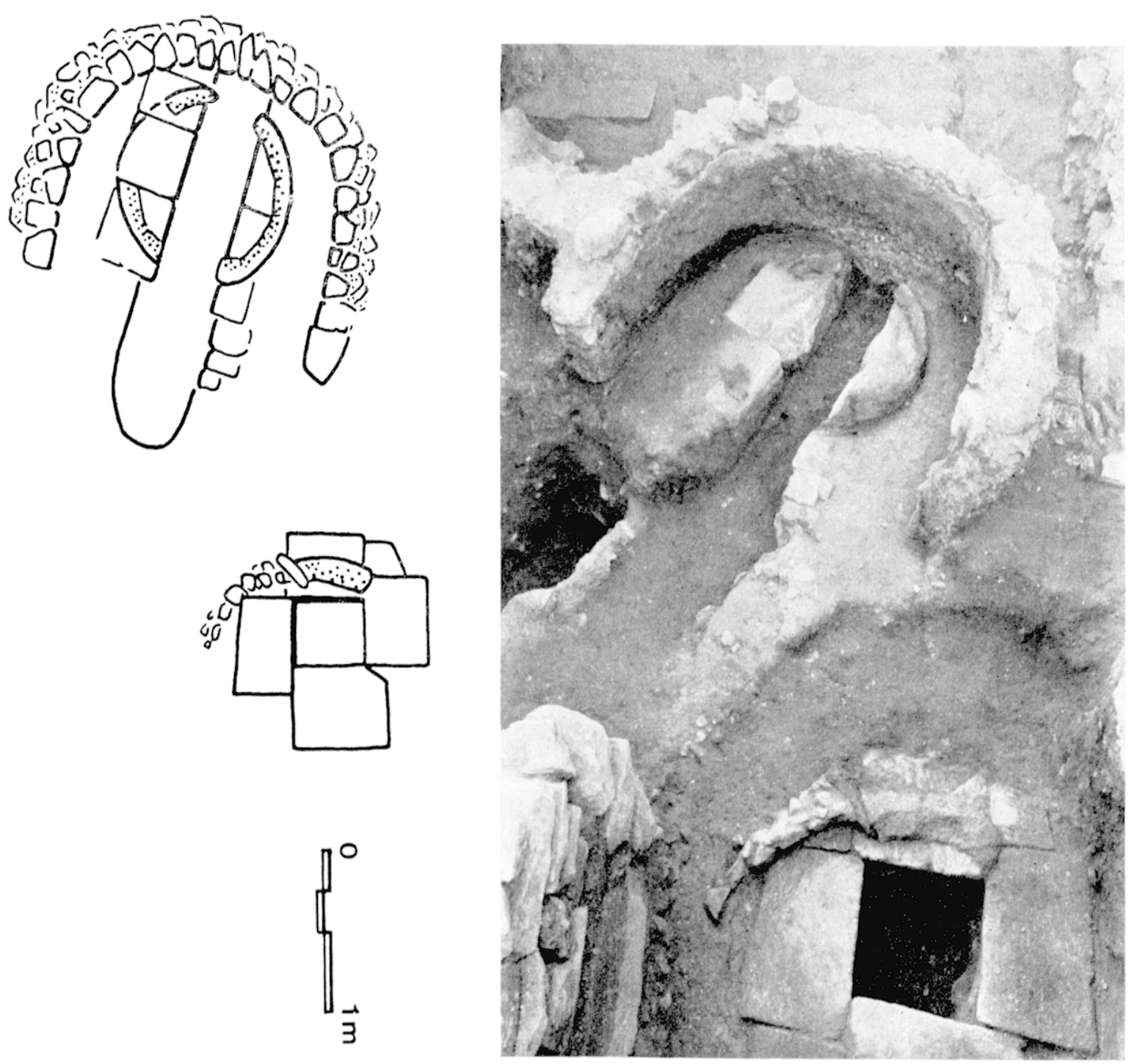

Fili. 67.

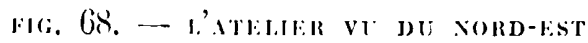

PLAN J)E LATELIIER DE: FONDETR STTUÉ A I'EST DF: LA COUR

la fusion du métal. Iin revanche un tel emplacement a éti découvert dans l'angle nord-ouest de la cour dans une tranchée de récupération de pierres, sur le traci d'origine antigur du mur 13 qui avait limite la cour du palais roman. Il est forme d'un petit alandier ovoïde de 1 mètre de longrueur sur $0^{\mathrm{m}} 80$ de large (fig. 45 et 69 ). Il a été retrouví avec un comblement de cliarbon de bois très pur. A l'extrémité méridionale du plus grand diametre une sole était construite au niveau du sol de tui-

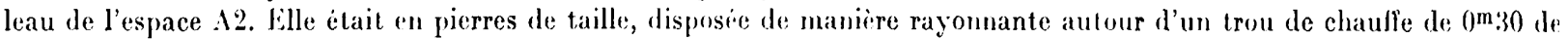
diamètre. laa sole et l'intérieur du trou de chanffe élaient tapissćes d'une pellicule d'argile.

Mais l'absence de cuisson de l'argile laisse sceptique sur son fonctionnement, même si le charbon de bois prouve que le feu a bien iti allumé.

Un cinquième emplacement a été découvert dans la partie orientale de la cour, destine celui-là a la fabrication de chaux (fig. 19). Le four grossierement circulaire, se présenlait sous la forme d'une fosse de t mètres de diamètre. Le four n'avait pas été entierement vide de sa charge apres la calcination du contenu. L'ensemble n'en a pas éti fouillé. Les parois

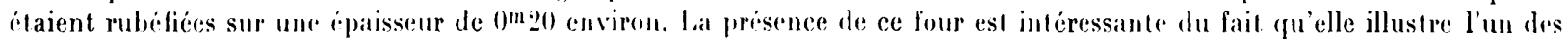
aspects des chantiers de construction et explique la recuperation systematique des pierres des idifices anterieurs et l'etat avancé de bien des destructions. Si clles no rataient pas remployées telles quelles, les pierres pouvaient done recevoir, sur place, d'autres utilisations. 


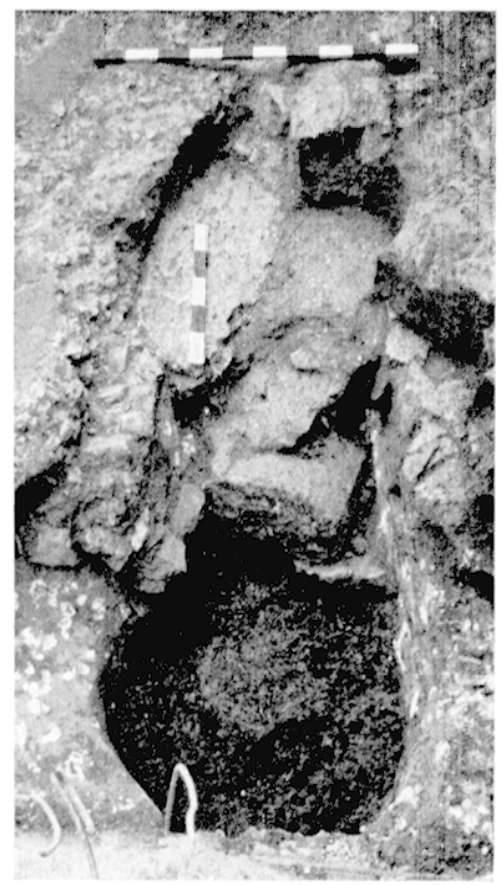

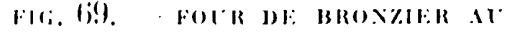 (1)R-(1) EST bl: l. lotr, I

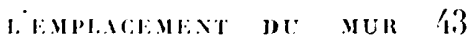

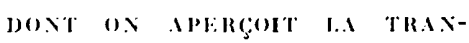

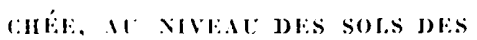

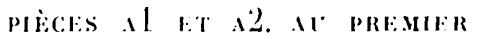 PIAX. PIOT lix BÉTOX ARME DI: THËTRR: DI: CASSANDRI:

\section{c) La datation du palais gothique.}

(lu a commencé a evoquer plus baut l'image d'un palais archiépiscopal datant de la fin du Moyen Age sur l'existence duquel les historiens de la ville se sont longtemps interrogés. Il était constitué d'une aile d'apparat, au nord, avec grande salle et chapelle adjacente a l'ilatre, sur un rez-de-chaussce dont on ignore la destination. Ia construction de cette aula serait contemporaine, selon l'interprelation proposie, de modifications intervenues dans l'aile sud qui, jusque-là, avait pour fonction de concentrer les reserves alimentaires. lin l'absence de fouilles, on ne peut que se demander s'il faut elablir une relation entre le connblement des silos et la conception d'un cellier au rez-de-chaussie de la sala. De l'état roman, seule cette aile sud demeurat. L'aile orientale, cutre-temps, avait éti détruite pour laisser la place à la conception d'une autre aile monumentale obéissant aux normes de l'architecture résidentielle bien représentée au xrye siècle dans la région, qu'il s'agisse des palais des evègues, des cardinaux ou du pape 1881. Appupies à la sixième travie de l'aula, les trois travées d'un promenoir voûté, portant un itage, fermaient la cour du còté du rempart, le long de la portion dite recta linea en 1292. De cette manière, le palais s'alossait sans doute à lit muraille du Bourg Saint-Sauveur.

Quelles dales suggère la fouille, en particulier, pour l'aile orientale qui achève le palais gothique?

Ln premier indice pourrait être fourni par l'abandon des salles de l'aile romane, abandon préalable à la destruction. Il existe en effet des données susceptibles de préciser la fin de l'utilisation de la cheminée adossée au mur 85. Cette désaffectation est matérialiste chronologriquement par le bris, sur place dans la cendre, d'un objet entier, une cruche en pâte claire portant une glaçure jaume, vaisselle représentée dans les périodes B2 et Cil de la fouille de Rougiers c'est-à-dire à la fin du $\mathrm{x}_{11} \mathrm{e}^{\mathrm{e}}$ sieccle et dans la première moitii du siècle suivant (89). Tne telle datation est confirmée par le catalogue des céramiques issues de la fouille des deux fours à cloche, implantés aux dépens de l'ancienne aile orientale.

Dans les deux cas, aucune céramique qui soit apparue après le début du xrve siècle ne peut être identifiée. Les céramiques communes glaçurés sont toutes du tỵpe à pâte claire, à l'exception d'un exemplaire de poterie à pâte rouge et glaçure plombifère, d'un tỵpe déjà mentionné ici pour se trouver associé avec des milieux exclusifs de céramiques à pâte grise. Parmi les céramiques fines, sont uniquement reprisenties des tessons de cruches à pâte claire et glaçure ocre-jaune, mal connus jusqu'ici mais en séries assez abondantes dans le matériel de cette fouille. (quant à l'autre four, situé au sud de la cour, il présente encore une grande prédominance de pâtes grises.

C'est donc, au plus tard, dans la première moitié du xrve siècle qu'il faut situer la disparition de l'aile orientale romane avec ses aménagenents intirieurs réalisés deux ou trois générations plus tôt. Cette destruction ne concerne pas le mur 85 qui, apris a voir séparé l'aile est de l'aile sud, divise maintenant de la cour l'extrémité des bâtiments méridionaux.

Vers le nord, la construction du promenoir nous a paru liée à un sol situé à la cote 204,20 . Les terres sous-jacentes à ce niveau ne contenant que quelques fraqments de céramique commune à pâte rouge, c'est donc sans doute à la première moitic du $x_{1} v^{e}$ siecle qu'il faut rapporter cette réfection de l'aile orientale. 


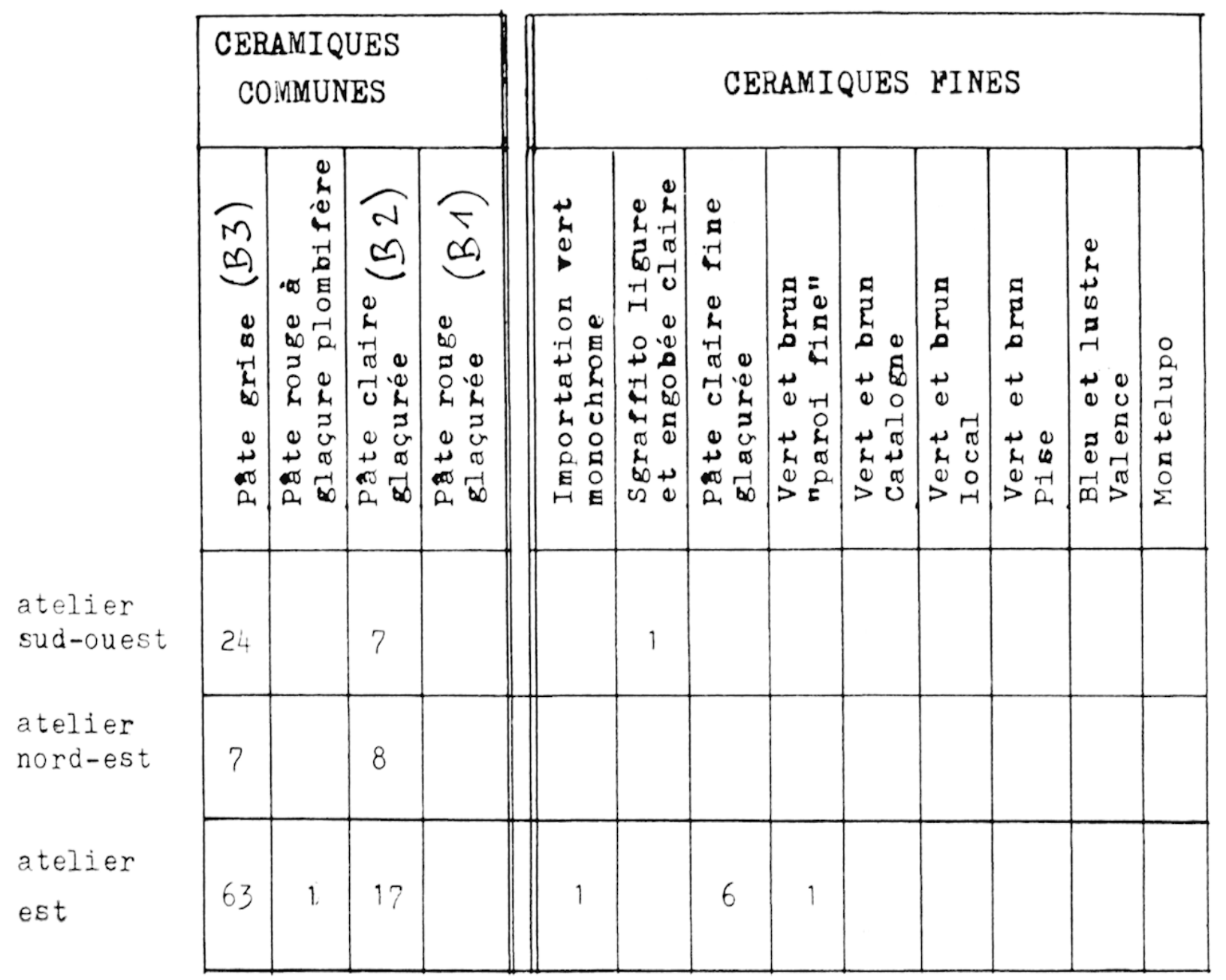

Tableau XV. Répartition des céramiques dans le comblement des ateliers de fondeurs de cloches.

Quant au four à chaux, il semble avoir été aménagé à une date plus tardive encore, puisque la partie supérieure do son comblement associe céramiques glaçurées diverses à pâte rouge, productions locales décorées au vert de cuivre et brun de manganèse, et importations valenciennes décorées au bleu de cobalt et au lustre métallique. Il appartient aux réfections postérieures à la fin du xıv ${ }^{\mathrm{e}}$ siècle, c'est-à-dire à la date à laquelle les historiens aixois ont longtemps situé les "fondements du palais 1.

Sans doute faut-il encore une fois revenir sur ce point. J. Pourrière a inontrí que de même que l'archevêque disposait d'un nouvel hospitium à l'emplacement du palais actuel dès le commencement de la seconde moitié du xirr ${ }^{\mathbf{e}}$ siècle, des réparations ou des travaux sont aussi attestés vers le milieu du siècle suivant, travaux interrompus daus la seconde moitié du $x_{I V}{ }^{e}$ siècle (90). C'est au cours de ce siècle d'ailleurs que les archevêques cherchèrent à rapprocher leur résidence du Bourg Saint-Sauveur afin de réunir les différentes fonctions d'un palais, non seulement lieu d'apparat, de représentation et d'administration, mais aussi résidence privée. Avant cette réunification l'archevêque put résider dans une maison qu'il possédait dans la Rue Droite; cette demeure se trouvait déjà tout près de la cathédrale, en vis-à-vis de l'entrée actuelle du palais, à l'emplacement qu'occupe maintenant l'hôtel Maynier d'Oppède. Pour se rapprocher, il vécut même dans la maison du prévôt, mais ce ne sont là que péripéties par rapport à une réalité qui est l'existence d'un lieu monumental, expression du pouvoir épiscopal, où fonctionne la curie pendant l'époque gothique comme pendant l'époque romane, et peut-être même avant celle-ci. L'évêque ou l'archevêque partageait son temps entre ce lieu et sa résidence des Tours, entre autres.

Semblable séparation, ou dissociation, n'est pas exceptionnelle. On peut se demander si l'évêque d'Apt, par exemple, n'a pas un certain temps vécu de la même manière entre un lieu où fonctionnait la curie, proche de la cathédrale et un autre, fortifié comme à Aix et situé aussi dans la ville, la Turris episcopalis, sans compter, dans un cas comme dans l'autre, une résidence suburbaine qui n'est pas en cause ici (91). Cette dissociation assez fréquente semble avoir pesé sur l'historiographie locale au point de faire méconnaître la réalité monumentale des constructions proches de la cathédrale. 


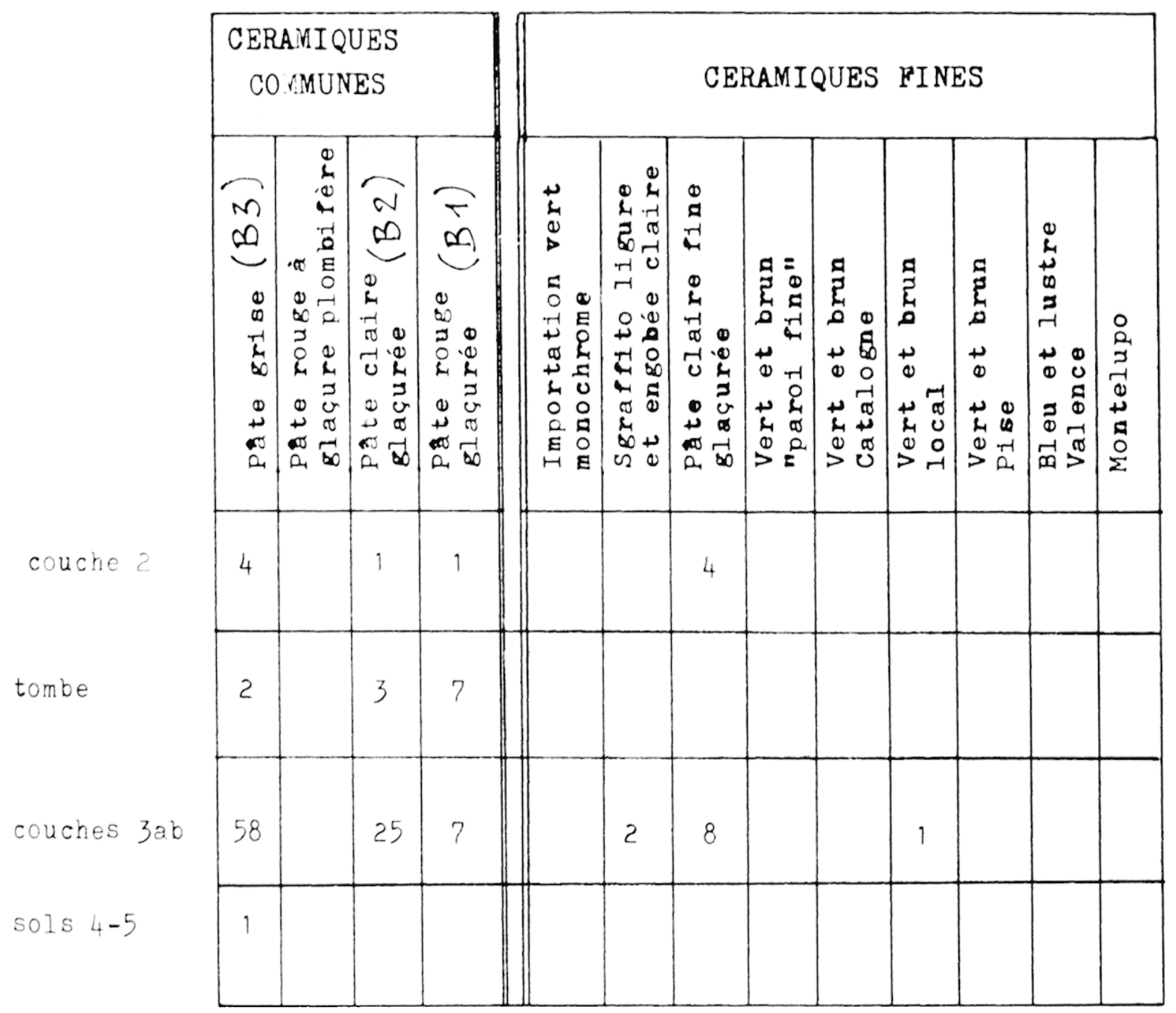

Tabreau XVI. Répartition des céramiques découvertes dans le sondage réalisé dans la tour de Pierre le Filleul.

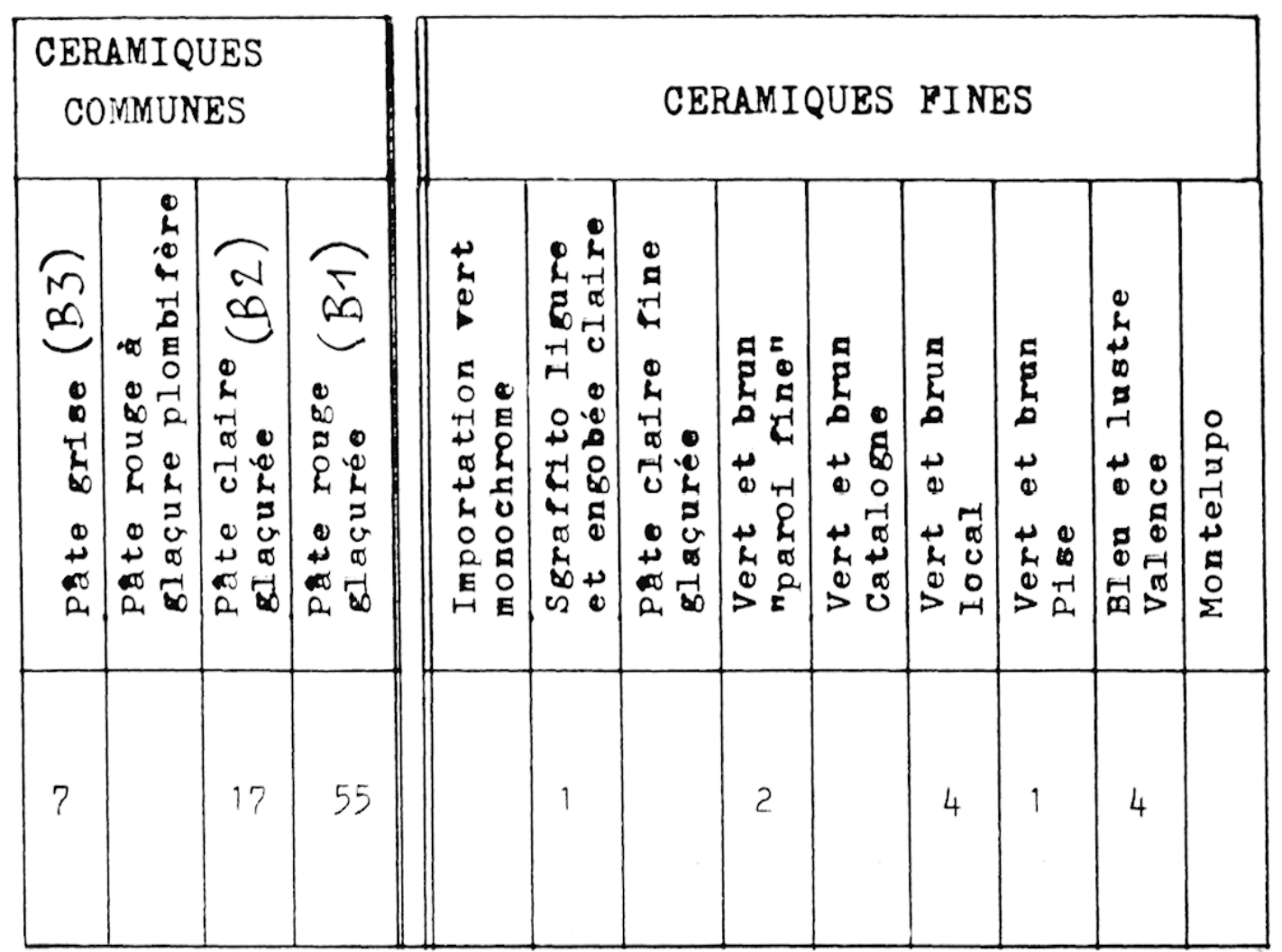

TABLEAU XVII. Répartition des céramiques découvertes dans le comblement du four à chaux. 


\section{III. - VERS I.'ÉPOQUE MODERTE}

Notre travail n'avait pas pour but d'étudier le palais a travers les temps, d'autant qur pour l'aporue muderne, il est assez bien connu. Cétait done surtout le problème de sa genese qu il paraissait intéressant de résoudre. Mais la présence d'un spécialiste des Temps Mfodernes n cut pent-être pas été inutile, parfois, pour ruregistrer des données au moment de la destruction des écuries de Charles de Vintimille, ou au moment de la refection ret du remodelage intérieur des pièces de l’aile est, dont tous les volumes ont été repris, at les murs décroûtés, à l'exoreption malheureusement de la partie médiévale. La fouille napporte done ici que quelques matcianx complementaires susceptibles d'enrichir un dossier.

\section{a) La réorganisation des parties sud de lu cour : du mur crénelés aux nouvelles écuries.}

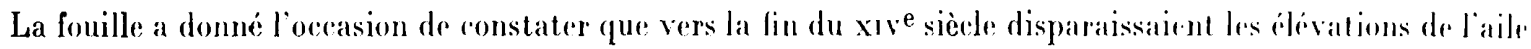
sud et que les niveaux d'occupation du courant du siècle noutaient pas remplaces. Cerla rorrespond a co que lon sait de l'abandon ou de la ruine de certaines parties du palais. Je manvais étal d'ensemble est invorque an commencement du xve siècle, en 1424, pour justifier linstallation de l'archevếque, sans doute Avignon Nieolaï, dans la maison du prévôt à qui était domnée, en échange, la demeure de la Rue J)roite. Je plan de Belleforest, dresse en 1573, premier document iconographique concernant la ville d'. lix, se fait le temoin de la simplification radicalo des parties méridionales (92). Ise palais arehiepiscopal n'est évidemment qu un détail dans cette vur gémírale. Il n'a pas partieulièrement intéressé le dessinateur, moins en tout ras que la cathédrale voisine, que la mouvelli enceinte avec ses tours, moins que la tour de la ville ou que certains couvonts fig. 70 . C̈est donc avec prudener: qu’il faut se servir de ce document qui noffre a priori pas plus de certitudes, pour son exploitation, que les dommés archéologiques avec lesquelles il faut maintenant le confronter.

Sur le plan, le palais, vu du sud, est organisé en trois ailes bâties autour d'une cour ecentrale planté diarbres. Au sud, un mur crénelé ferme l'espace. Laile nord est representie sans etage. Fille est entièrement aveugle. Cela ne serait pas gênant, on l'a vu, si seul le rez-de-chaussée etait alors eomservé. lin simple graphisme marque le mur et la toiture. C'est une image étrange si les deux niveaux existaient encore, car le dessinateur nia jamais hésité, au palais ou ailleurs, dans la vue rapide des maisons, a représenter des ouvertures, fenêtres ou portes. Deux baies, en revanche, animent la façade de l'aile orientale représentée elle aussi sans étage. J)ans l'une et l'aut re partie, certte absence est bizarre compte tenu de ce que nous savons des modeles architerturaux que reproduisent res ailes du palais, et aussi par rapport à la visite de 1708 qui décrit bien lesétages voùtés d'ogives, ainsi que la chapelle adjacente. Jais si l'on prête attention à ces seules ouvertures de l'aile orientale, on remarque une organisation con deux travées. Une troisième peut être restituée, dissimulée par limplantation d’une tour qui a fait son apparition dans l'angle nord-est de la cour, en avant des façades. On retrouverait bien alors les trois traveres du promenoir. Majs faut-il aller si loin dans l’interprétation du dessin? Serait-il a la fois précis pour crertains traits ot indifferent pour d'autres?

Sur le même dessin, en direction du sud, l'aile orientale s'articule avec un mur crénelé, par l'intermédiaire d'un mur méridien joignant aussi deux corps de maison organisés en équerre, au sud du mur crénelé, dans un espace de jardins. Dans cette partie, l'absence de la rue des Ayguesiers doit renforecr motre circonspection 993 .

Quels éléments retrouvent leur place dans une confrontation avec les domnés archéologiques? C"est sans doute, et de manière assez certaine, le mur crénelé dont la présence consacre la disparition de l'aile sud du palais roman, en rayant d'un trait la surface qu'elle occupait fig. 71 . Cette partie de commuss est finalement, parmi les trois ailes que nous connaissons, la dernière à avoir subsisté dans son état roman, tandis que les ailes mobles avaient été plus vite remplacées.

La construction du mur crénelé marquait donc un aboutissement dans cette évolution de la fin du Moyen Age; il donnait au palais une image monumentale finie, en faisant disparaître derrière un ceran co qui pouvait rester de communs trop ostentatoires. On peut proposer lidentification de ce mur énele aver une fondation retrouvée sur 5 mètres de longueur seulement a $0 \mathrm{~m}^{5} 0$ au sud du mur de la façade romane de laaile sud mur 71 . Aussi la surface de la cour ancienne ne fut-elle pas sensiblement modifiée par cette nouvelle construction. Vers l'ouest, la fouille n'a permis que d'en reconnaître le négatif. I la hauteur de som arase supérieure conservée, le:

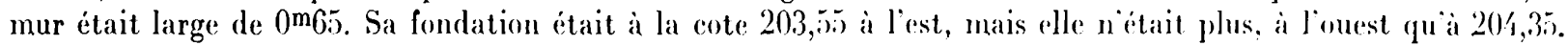
Ja construction dut être accompagnée d'un apport de terre pour finir de discimuler les ruines romanes. Au plus haut niveau observable, a 204,25, on se situait encore a la hauteur d'assises de fondation. De constitution assez légère, ce mur semble avoir surtout été conçu de manière ostentatoire. Vers l'est, il s'achevait en surépaisseur à un point de rencontre de trois autres murs. Cette jonction s'opérait en un endroit qui, depuis l'époque romane, constituait une zone d'articulation ancienne entre l'aile sud ot l'aile est.

Parmi ces murs retrouvés, il en est un second, le mur 110, qui paraît identifial)le sur le plan de Belleforest. 


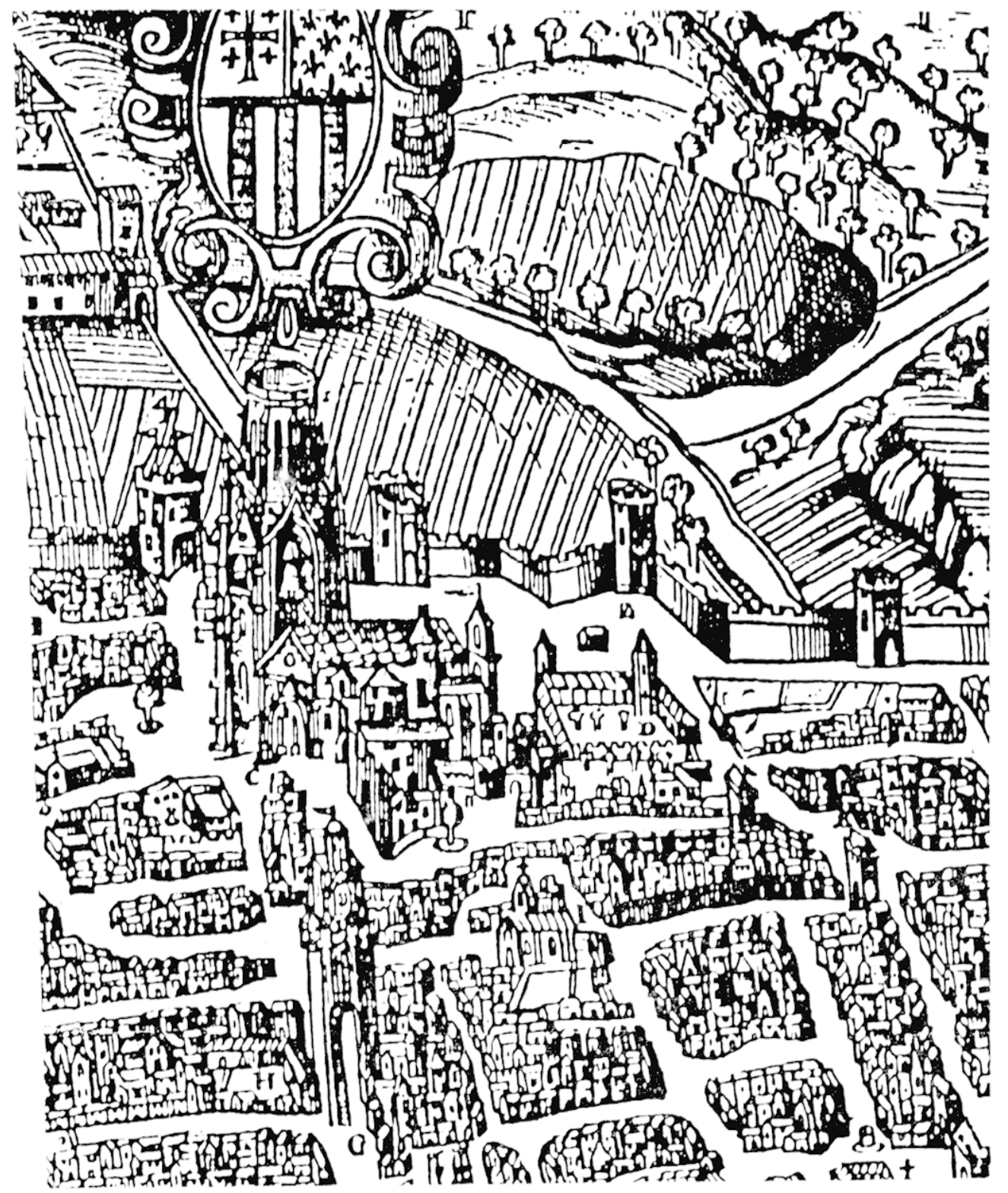

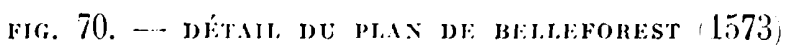

MONTRAYT IE QUARTIER DE TA CaTHÉDRALE

C'est celui dont on voit nettement le tracé, perpendiculaire a celui du mur crénelé. line fondation puissante de direction méridienne a été dégagér. Son arase culminait à la cote 201,55. Cette altitude, qui correspond encore à relle de niveaux de fondation, confirme l'apport de remblais au-dessus des sols des anciens communs détruits. La largeur, 0m90, est plus importante que celle des arases supérieures des fondations du mur crénelé. Vers le sud, re mur rejoint le mur 9' qui appartient au système gothique. Mais il tranche toutes les autres constructions rencontrées, en particulier les murs 86 et 91 , contemporains du mur 9'́. Or, sur le plan de Belleforest, cre mur 110 rejoint aussi, vers le sud, deux corps de bâtiment orthogonaux - peut-être trois - - représentés aver: une précision qui n'est pas l'effet du hasard, car ils se distinguent nettement des autres maisons du voisinage.

Il faut chercher a situer ces bâtiments par rapport aux nouvelles écuries de Charles de Vintimille, dont

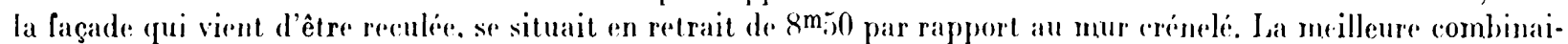
son pessible des donnés de fouille et du dessin fait coüncider le mur nord du groupe de bâtiments en question avec le mur 9/, lequel s'articule en effet avec le mur 110.

La façade occidentale de ces maisons pourrait, alors, correspondre encore à une ligne directrice de l'urba- 


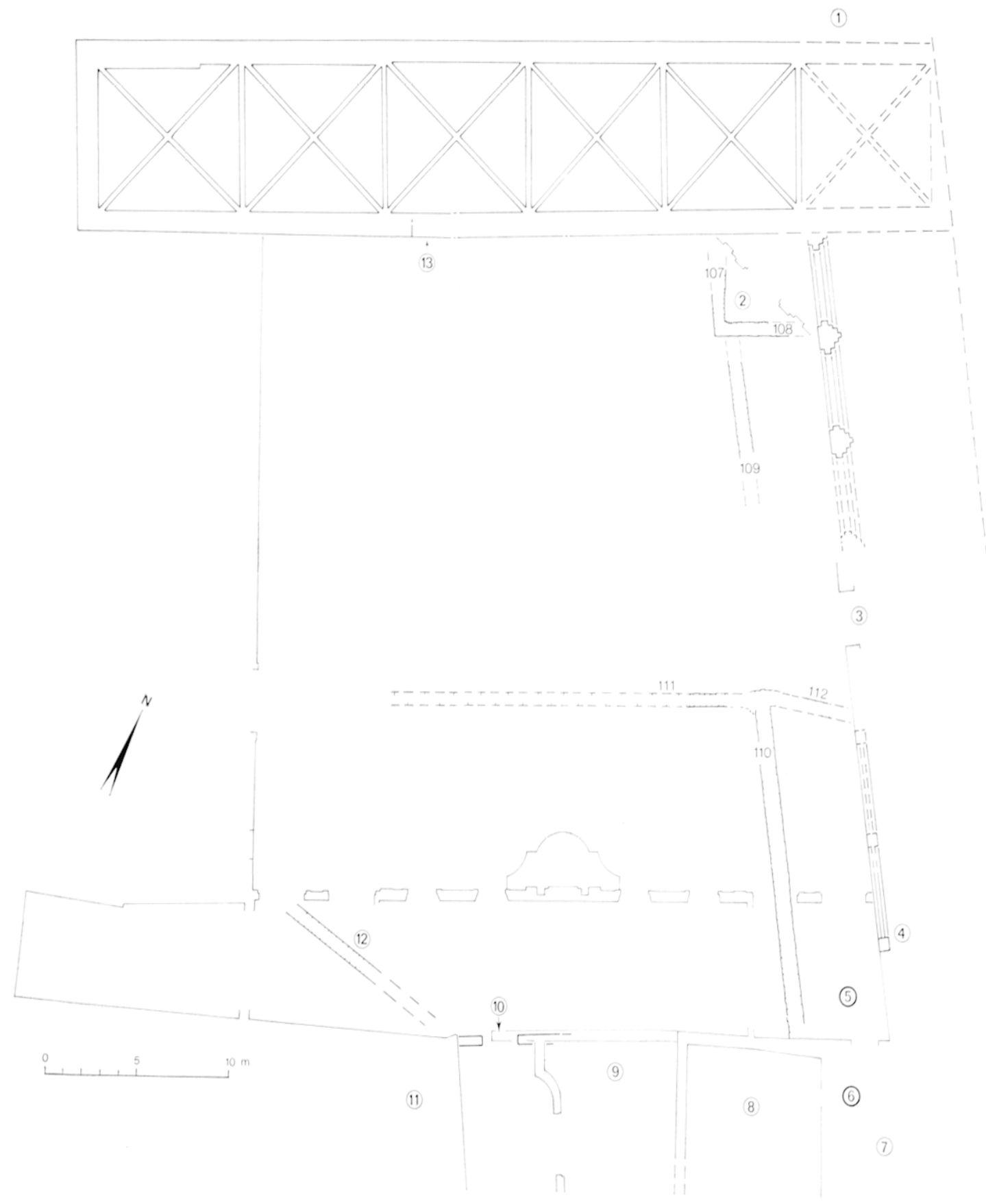

FIG. 71. - RFPÉRAGE DES VFSTIGIS DE I ÉPOQLE MODERNE

1) emplacement de la chapelle; 2 tour de l'escalier d'angle attribuce a Pierre le Filleul ; 3 f emplacement d'un premier accès oriental a la cour du palais ; 4) pile et arcature reprise dans la façade orientale donnant sur la cour : j) et 6) puits ; 7) emplacement de la maison d'A rguillosy ; 8/ maison tabre ; 9/ cave sous le lavoir ; 10) porte méridionale antérieure à la construction des nouvelles écuries et situce dans l'axe de la rue des $A$ yguesiers ; 11) Ifôtel des Ayguesiers; 12 mur oblique de limite retrouvé sous les nouvelles écuries. 


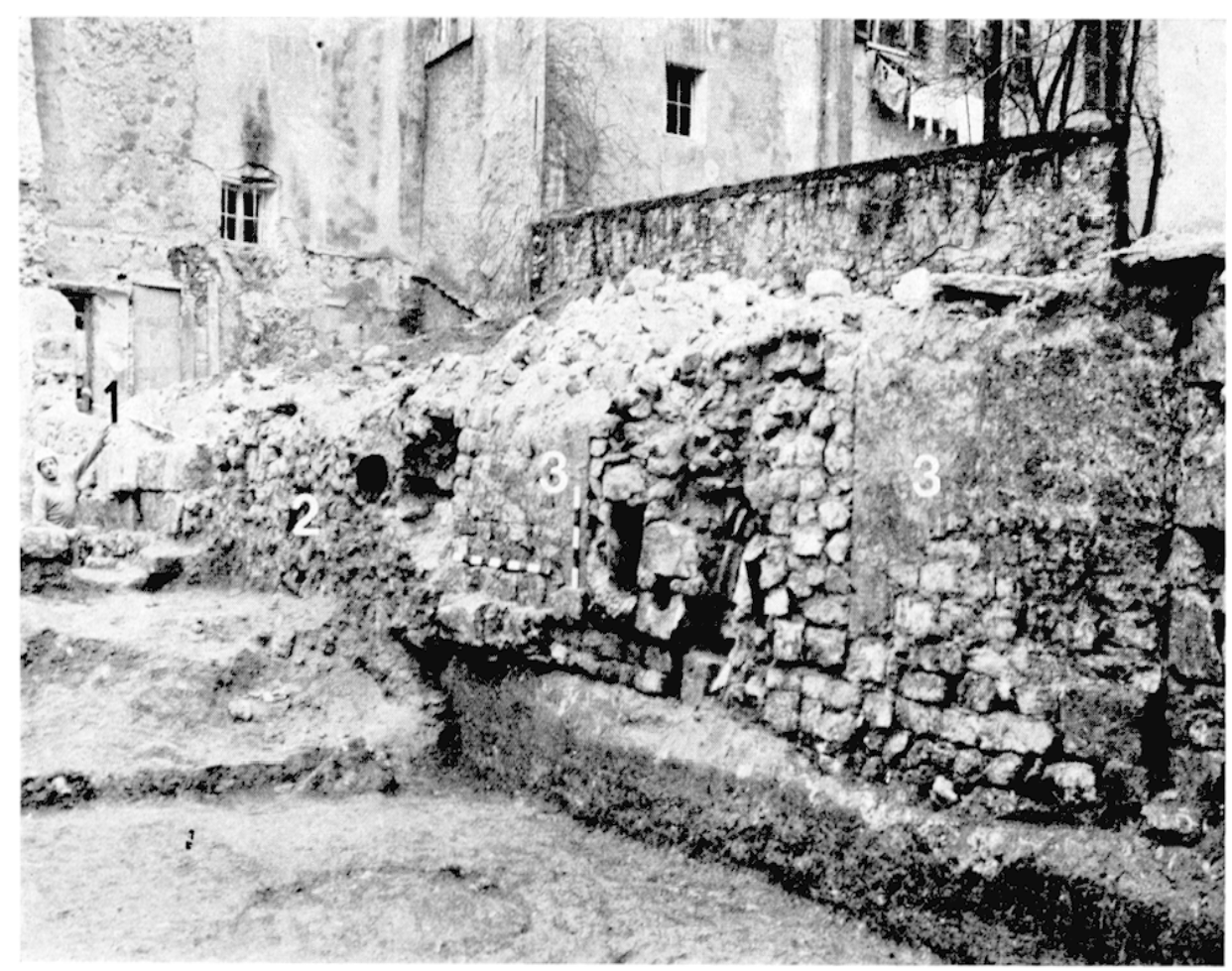

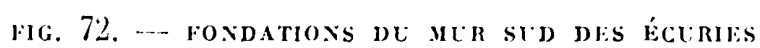

1) blocs de libage superposés au mur medieval 9' ; 2) revers du mur de la cave supportant le lavoir ; 3) mur avec porte charretiere au-dessus du massif de beton hydraulique en façade nord de l'insula II (cf. fig. 5).

nisme antique. Avec des conditions de fouille assez médiocres, dans des terrains très remaniris où aucun niveau médiéval ne subsistait, on s’est aperçu que le mur \$6, mur de la façade orientale de l'insula sud, avait été réutilisé comme fondation d'une élévation non datable, mais dont la disposition etait mitoyenne, entre une maison avec cave et un espace adjacent à l'est. Ainsi, en dehors des limites du palais médiéval, dont les agrandissements successifs avaient progressivement gommé le canevas antique sous-jacent, ces lignes directrices anciennes réapparaissent, respectées encore par des maisons de particuliers, moins susecptilles sans doute d'occuper l'ancien espace public, ou du moins prenant un certain retard dans le processus d'aliénation. Du corps de bâtiments représenté sur le plan de Belleforest, il deviendrait donc possible par la fouille d'identifier deux murs orthogonaux, l'un étant la façade nord constituée par le mur 94, l'autre la façade ouest élevée au-dessus du mur 46.

Il suffit maintenant, pour obtenir confirmation de cette interprétation, de se laisser conduire à travers la partie méridionale du palais par les visiteurs de 1708 et de 1729 qui expertisèrent les bâtiments à la mort de Daniel de Cosnac puis lors de celle de Charles de Vintimille, c'est-à-dire avant et après la construction des nouvelles écuries. Chemin faisant, quelques aspects du palais moderne réapparaîtront, révélés par la fouille. Ein 1729, entrés dans l'aile sud par une " demi-porte ", les visiteurs empruntent un passage pavé de cailloux, où l'on reconnaît le couloir oriental du bâtiment détruit, avec sa calade à la cote 205,20 en continuité avec celle de la cour. Ils se trouvent alors au pied de l'escalier rampe sur rampe dommant accès à l'étage de l'aile orienlale, dans un espace à ciel ouvert. Ils décrivent un puits, qui a été retrouvé (fig. 71, no 6). Ce puits et sa margelle, disent les visitcurs, ont été faits "aux dépens de Monscigneur l'Archevêque de Paris en remplacement d'un autre qui était dans la grande cour ». De fait, cet autre puits a été aussi retrouvé sous le passage oriental dont il vient d'être question ifig. 71, no 5 ). Il fut obturé par conséquent lors de la construction des nouvelles écuries. La phrase indique par ailleurs qu'après la destruction du mur crénelé, à une date que l'on ignore, mais sans doute dans le courant du xvin siècle $(94)$, la cour s'est trouvée agrandic. Ellle fut diminuée à nouveau par l'implantation des nouvelles écuries.

"De là, contimuent les experts, nous sommes entrés à une cour où l'on repose le fumier, par un passage-que nous avons souvent emprunté au cours de la fouille -- qui est dans une des maisons acquises par Monseigneur l'Irchevêque de Paris de la dame d'. Irguillosy... par acte du \& septembre $1721 \ldots$ et une autre acquisition de Monseigneur de Paris, de Pierre Fabre, d'une maison attenante à celle de la dame d'Arguillosy le 16 mars 1720 ... Cette basse-cour est composée de partie du sol de maison de la dame d'. Irguillosy, de tout le sol de la maison du dit 


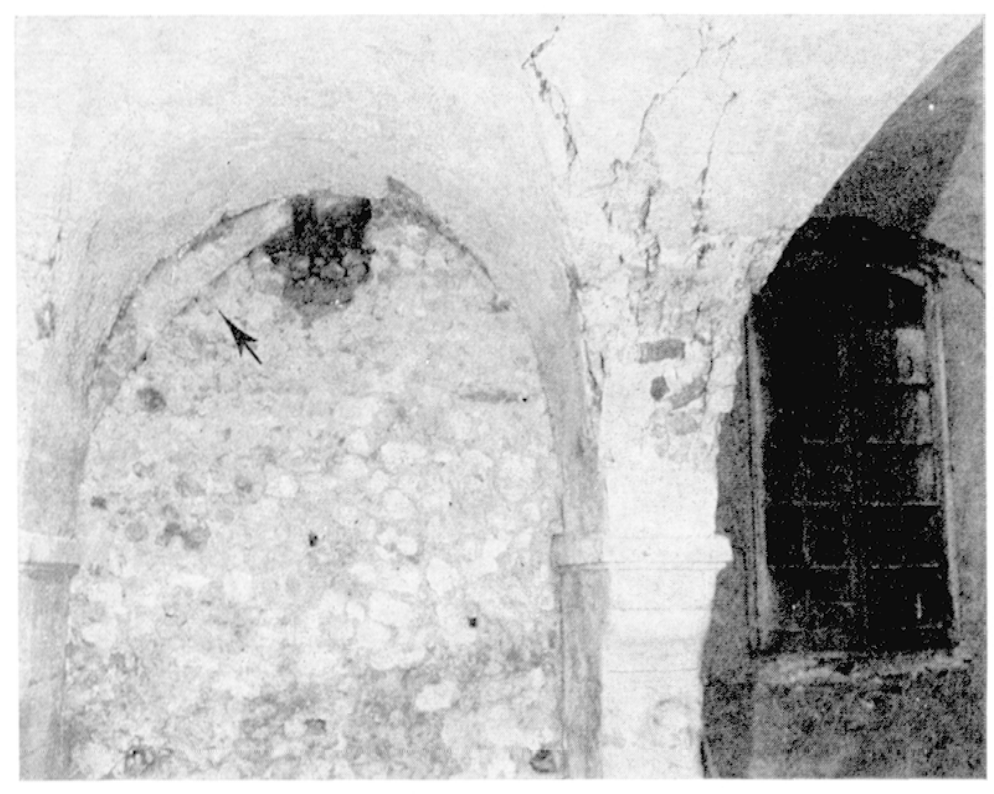

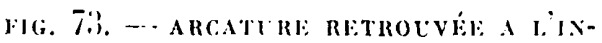
TERHETR DU MLR ORILXTAL DE: T.A

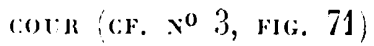

Fabre qui a été entièrement abattue et du sol d'un petit bâtiment qui ètait de l'archevêché et qui servait à l'usage des lessives abattu par l'ordre de. Monseigneur de Paris ". Or par la visite de 1708, nous savons que ce lavoir alimente par la surverse de la fontaine qui était à cette date au centre de la cour, se trourait au-dessus d'une cave, certainement la cave retrouvée, puis détruite aussitoot en 1985) qui jouxtait au sud les nouvelles écuries (fig. 71 , $n^{0}$ 9. Aussi la disposition antérieure a la construction des écuries parait-elle restituable : le noyau de la maison de la dame d Arguillosy préservée en partie paraît bien correspondre à ce qui, sur le plan de Brileforest, est dessiné sous la forme du hâtiment est-ouest, dont le mur nord est sans doute le mur 9/1. lit c'est la fagade occidentale de la maison labbre qui sölevait sur le mur antique -46 séparant cette propriété de la cave, dépendance de l'archevêché. Pour la construction des nouvelles écuries, Charles de Vintimille fit réutiliser, pour fonder le mur sud, le mur gothique 9', anciemne limite des dépendances du palais depuis que celui-ci, dans le courant du $\mathrm{xu}^{\mathrm{e}}$ siècle, avait compris le tracé de l'ancien decumanus dans ses espaces couverts. Déjà, cette surface devait être annexée, comme cour, à l'épooque de l'aménagement des silos. C"est contre re mur 9', toujours mur de limite, que s'était appuyée, au sud, la maison appartenant au début du xvme siècle à la dame d'Arguillosy. Sur cette maģonnerie ancienne, la reprise, commencée vers 1715, se marqua par la mise en place de deux très grosses assises de libage, en pierre de Rognes. C'est de la même façon qu'avait été fondé l'appui des baies percées dans la façade de l'aile nord par Daniel de Cosnac.

Au-delì de l'ancien angle sud-ouest de l'espace VIII, encore matérialisé par une chaîne d'angle posé en fondation sur ce qui restait ici du béton antique, un court tronçon de fondations nouvelles fut mis en place, en liaison entre l'alignement d'origine gothique et un autre alignement existant aussi déjà, appuyé sur le rebord de l'égout antique longeant le mur 55 (fig. 72). Ainsi, à l'extérieur de l'emprise des constructions de l'ancien palais roman, comme dans le cas du mur 42 , l'alignement existant au commencement du xvir ${ }^{\mathrm{e}}$ siècle, reprenait encore, à peu de choses près, le tracé antique du decumanus. Avant les récents travaux, dans la cour sud-ouest, qui était l'emplacement du bûcher, le mur sud rappelait encore cette vieille disposition. Cet espace, déformé, évoquait l'annexion de la voirie antique par des cours et des espaces ouverts, dépendances du palais. Ainsi, la partie oceidentale du mur sud des nouvelles écuries reprenait une ancienne élévation, mur de limite peut-être, plus que de bâtiment, percée par une porte charretière (fig. $71, n^{0} 10$ / qui fut alors murée. Celle-ci faisait communiquer la rue des Ayguesiers avec des offices qui semblent avoir existé là encore en 1708. Aménagée dans l'axe de la rue, sa construetion avait sans doute consacré l'abandon de l'ancien passage issu du portique.

Dans cette rígion, mal documentée archéologiquement, les nouvelles écuries franchissaient le mur oblique (fig. $71, n^{\circ} 12$ articulé au sud-est à l'angle de l'Hòtel des Ayguesiers. On sait que l'orientation de ce mur est due a une déviation progressive du réseau des murs antiques; il pouvait marquer la limite de l'emprise du palais dans une région de l'ancienne insula qui échappait peut-être déjà à la dépendance épiscopale. lin dépit d'acquisitions faites non loin de là au xvise siècle par ses prédécesseurs, Charles de Vintimille eut qquelques difficultés pour joindre les nouvelles écuries avec l'aile ouest et construire le nouveau pavillon d'entrée. 


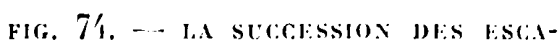

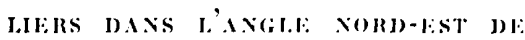
LA (c): R

1) escalier droit médiéval entre les plots de béton du thêtue de Cassandre; 2) fondations de la tour attribuce à Pierre le Filleul; 3) pavillon en pan coupé attribué à laurent Vallon; 4) mur 109.

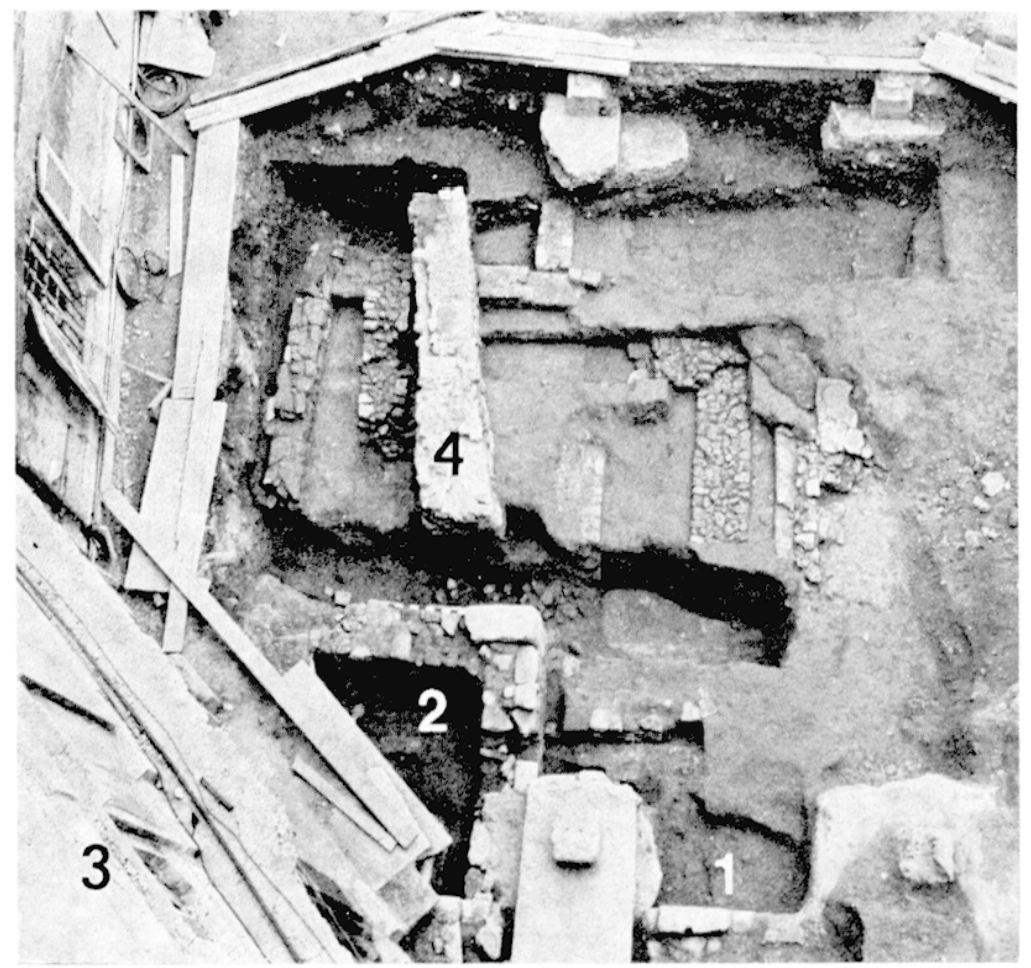

\section{b) L'aile orientale.}

L'exploitation du plan de Belleforest apparait moins facile en ce qui concerne l'articulation du mur crénelé et de l'aile orientale, encore limitée, semble-t-il, au promenoir. Sur le dessin, celle-ci paraît s'achever par un mur pignon qui se dresse à une certaine distance au nord du mur crénelé. Cette façon de voir correspond à la réalité archéologique, mais plus étonnante est la disposition du mur méridien par rapport à crtte extrémité de l'aile est. Il semblerait venir frapper dans l'axe du pignon, à moins que dans la tache noire qui marque le dessin à cet endroit on ne puisse restituer un passage vers l'est, passage précédant celui qui existe au début du $\mathrm{xvil}^{\mathrm{e}}$ siècle sur le plan de J. Maretz. C'est ce que pourrait bien suggérer la découverte, par la fouille, de la forme en tau de l'extrémité nord de ce grand mur méridien, près de l'endroit où il rejoint le mur crénelé. On imaginerait assez bien que ce qui a été retrouvé puisse correspondre au flanc méridional d'une telle porte, contemporaine d'une réfection rustique du mur 85 par le mur 112.

Au moment d'abandonner ces parages du mur crénelé, il faut avouer que la fouille de sa fondation n'a pas donné de critères pour le dater. Si le plan de Belleforest donne un terminus ante quem, l'autre terminus n'est fourni que par les niveaux d'abandon et de comblement de la fin du xıve siècle, au-dessus des bâtiments romans disparus. Il faudrait alors le dater au plus tôt des réfections dues aux archevêques du $\mathrm{xv}^{\mathrm{e}}$ siècle, Robert Damiani ou Olivier de Pennart. Le plan de J. Maretz semble encore indiquer la présence de ce mur divisant la cour du palais en deux parties. Il aurait alors perdu son crénelage.

Le même plan montre l'aile orientale prolongée dorénavant au sud de la porte qui ouvre vers le séminaire. L'espace est bien décrit lors de la visite de 1708 : « du passage nous sommes entrés dans les écuries... le sol est pavé de cailloux et le haut voûté à croisillons soutenu par des piliers de pierre de taille de chaque còtè ". Or, le décroûtage des murs a montré que cet état ne correspondait pas à la disposition primitive. $\Lambda$ la quatrième travée en partant du nord, un grand arc en plein cintre est apparu dans le mur ouest, arc dont le diamètre ne correspond pas au rythme des travées actuelles (fig. 73). On peut se demander s'il s'agit seulement d'une porte. Car compte tenu de la restitution proposée, dans la partie nord de l'aile, d'un promenoir médiéval ouvrant sur la cour par une suite d'arcatures, il est possible d'imaginer un premier agrandissement reproduisant vers le sud ce parti. Dans un deuxième temps serait intervenue la fermeture de cet espace et son voûtement "à croisillons ", ce qui sans la mention d'ogives ou d'autres arcs désigne la voûte d'arêtes qui s'y trouve encore. Ainsi, avant le xvin e siècle, la façade de l'aile est prenait son aspect définitif non seulement avec l'obturation de toutes les arcades. mais aussi l'adoption d'un seul alignement corrigeant les décalages entre les deux états précédents. 


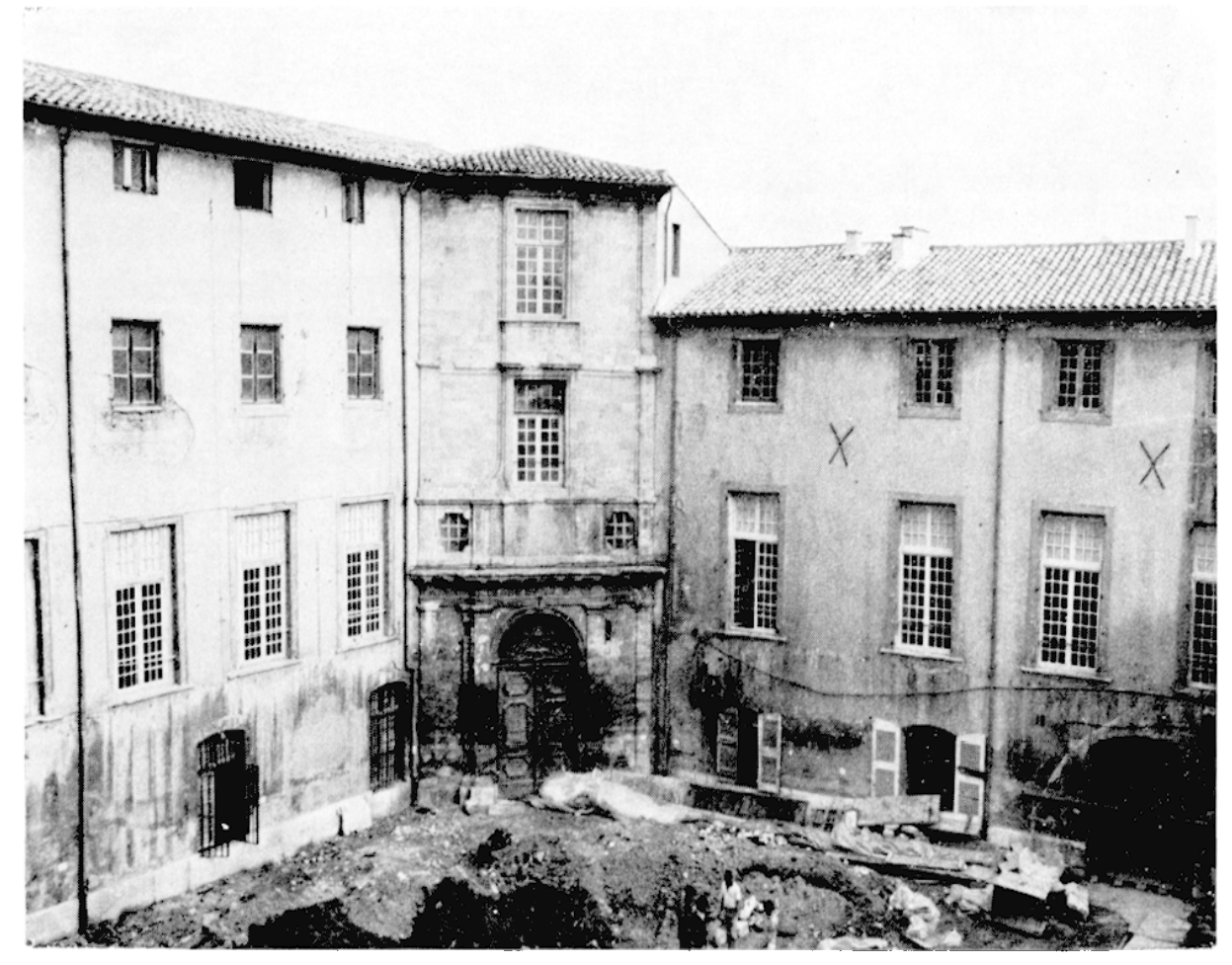

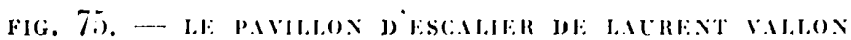
AL NORD-EST DF: IA COUR 1690 ,

\section{c) La tour nord-est.}

Les données concernant la tour de l’angle nord-est de la cour sont plus facilement utilisables, encore que le plan de Belleforest ne prenne une partie de son sens que grâce a la fouille. La tour est representír hors aruve, montrant, semble-t-il, trois faces, ce qui suggère un plan polygronal. (or il n'en rest rien. I l'emplacement préris indiqué par le dessin ont été retrouvées les fondations assez frêles diune construction de plan quadrangulaire et

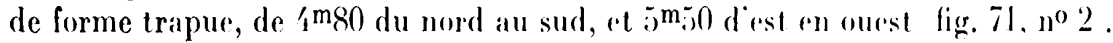

La fondation senfonce jusqua a la cote 203,35. Ville est en mollons lies au mortior. lípaisseur des parois

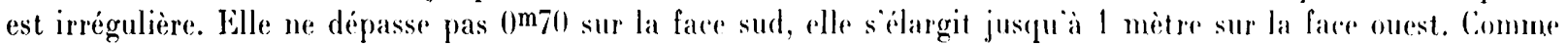
chaîne d'angle ont été remployés des blocs de pierre froide dorigine anticue, dont l'un à bossage.

Dans son implantation, ere massif tient compte du rythme des través du promenoir. Il sappuie sur liangle du ressaut interieur de la pile séparant lareature nord de l’areature médiane. On pent, sans ristue, lëdentifier

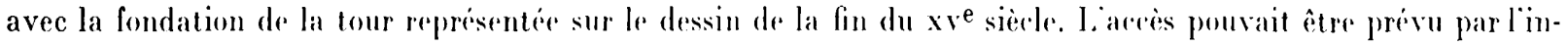
térieur du promenoir. Eat de fait, le dessinateur na pas represente de porte ouvrant sur la rour, alors qu il a mont ré des fenêtres à l'etage. Cette tour fut détruite a la fin du xvo siède pour laisser la place au pavillon deangle en pan

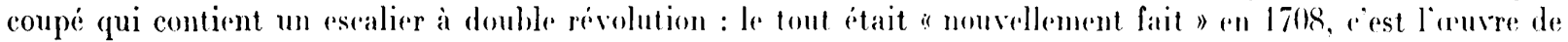
l'architecte Laurent Vallon i9j, vers 1690 lig. 75). Par le même document, on sait que la tour contenait un escalier, un belvédère et un pigeonnier. Liescalior a évidemment remplacé l'escalier droit médieval. Avee celui qui fut bâti par Daniel de Cosnace, et en tenant compte d'un escalier primitif en bois desservant l'étage noble de l'aula. on trouve là une singulière continuité et un resume de l'histoire de l'escalier nomumental, du xme an xvme sièrle (fig. 74). De quand datait la tour? Les magonneries irregulièrement assisées nont aucun caractère "médiéval ». Il est fort probable que la tradition qui en fait une auve de l'évêque Pierre le Filleul entre 1506 et 1541 soit exacte (96). On sait que les armes de ce prelat liguraient sur l'élévation. Compte temu de son implantation, rette tour laisse supposer que les deux dernières arcatures du promenoir médiéval étaient encore ouvertes.

La fouille a permis d’éclaircir en outre un détail du dessin. Contre la face occidentale, déjà la plus forte en fondation, a été dégage un gros massif de magonnerie dont l'interpretation peut etre domée grâce à lieonographie. Il s'agit sans doute du soubassement d'un gros contrefort appliqué sur cette face, ret dont la représentation sur le plan de Belleforest avait fait croire à une tour polygonale. lin revanche, napparait pas sur le document une maçonnerie appuyée à l'angle de cette tour, sur la face sud. C'est une fondation peu profonde de direstion méridienne, 


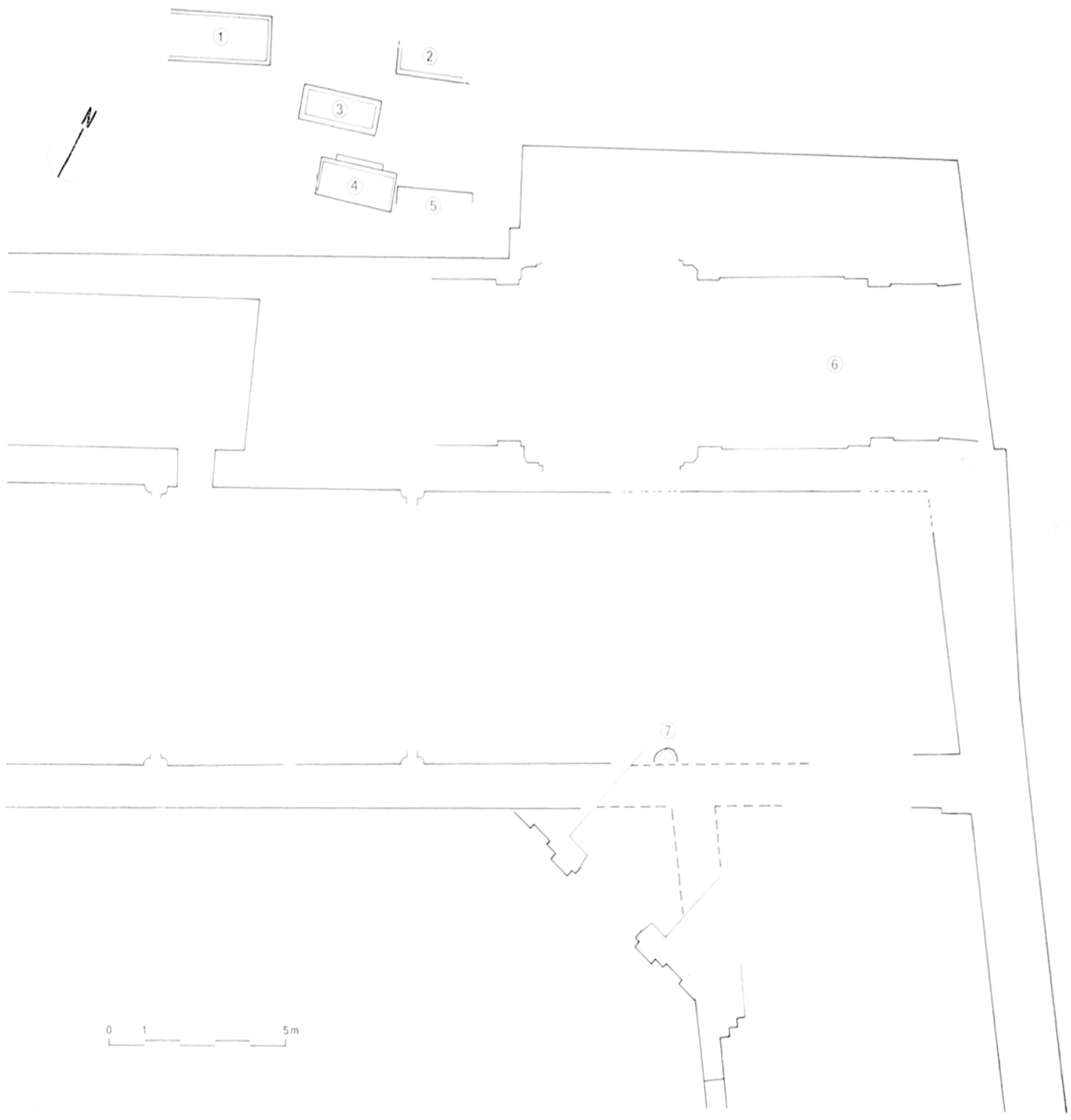

FIG. 76. - A.NGIE NORD-EST DU PALAIS

1) à 4) sépultures découvertes lors du sondage dans le cimetière Saint-Sauveur; 5) chaperon de mur en pierre froide ; 6) chapelle ; 7) trace de retombée d'arcs et mur reconnu en 1973 sous le pavillon d'escalier de Laurent Vallon.

le mur 109 ; elle a été retrouvée sur une longucur de 6 mètres. Son tracé est exactement parallèle à celui des arcatures du promenoir, à $3 \mathrm{~m}^{\mathrm{j}} 0$ en avant d'elles et se situe aussi dans le prolongement du mur méridien perpendiculaire au mur crénelé. Dans son démontage ont été retrouvés des éléments remployés de moulurations gothiques tardives, et une assise complète d'une pile polygonale. A quelle élévation correspond une telle fondation? Elle ne supportait pas un mur de façade. L'iconographie montre que celle-ci fut toujours à l'aplomb des arcatures du promenoir. S'agit-il, dans ce cas, de la trace d'un mur bahut destiné à porter une galerie couverte, en bois ou en pierre, qui remplaçait le promenoir médiéval une fois fermé (97)?

Une dernière trouvaille faite dans cette zone ne doit pas être passée sous silence. Elle a trait aux coutumes funéraires. En fouillant les sols à l'intérieur des fondations de la tour, une sépulture a été découverte. C'est celle d'un adolescent de dix-sept ans environ, vraisemblablement de sexe masculin. La céramique et la stratigraphie permettent d'en proposer la datation, en montrant son intercalation entre le temps de l'escalier droit et celui de 
l'escalier contenu dans la tour. C'est donc au pied de la rampe du premier que le corps a été déposé, vraisemblablement dans le courant du $\mathrm{xiv}^{\mathrm{e}}$ siècle (cf. tableau XVI). Sépulture privilégiée? Pourquoi un traitement spécial isole-t-il ce défunt de la communauté des morts, pourtant toute proche, au nord de l'aula?

\section{d) Un sondage dans la nécropole Saint-Sauveur.}

L'aménagement pour le Théâtre du Festival d'un escalier de secours donnant sur la face nord de l'aile septentrionale a fait ouvrir une étroite fenêtre sur le cimetière qui s'étendait au chevet des églises cathédrales. On sait que cette nécropole fut définitivement désaffectée en 1729 pour être mise en culture. En 1733, une bonne partie était accordée à l'archevêque pour agrandir son jardin. Mais déjà, dans le courant du xvir e siècle, ce cimetière avait été rétréci, soit par la construction de

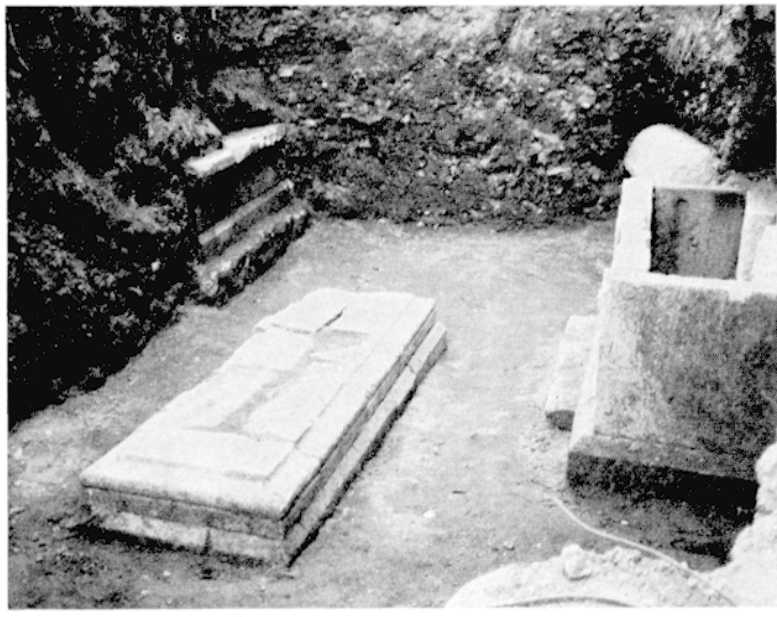

FIG. 77. - TOMBES ET CAVEAUX DANS LI: SONDAGE DU CIMETIËE chapelles au nord de la nef gothique de la cathédrale, soit par l'autorisation qu'avait donnée en 1656 le chapitre à l'archevêque Grimaldi d'en prendre une partie jouxtant la galerie nord du palais, pour en faire son jardin. C'est donc vraisemblablement dans la surface concernée par ce texte qu'ont été effectués les travaux (98).

La mise en culture a été à l'origine d'un important remblaiement au-dessus des niveaux de la nécropole. Le jardin a été établi pour être de plain-pied avec le premier étage de l'aile nord. Une couche de $1 \mathrm{~m} 80$ de terre cultivable a donc été rapportée. Puis à la cole 207,20 se trouve le sommet de terres contenant de nombreux ossements épars. C'est le niveau du sol au moment de l'abandon du cimetière. $\Lambda$ cette période correspond un certain nombre d'inhumations pauvres, en pleine terre. De fait, à l'aube de l'abandon de la nécropole au chevet des églises, en 1715 , c'est " pour les pauvres " qu'un nouveau cimetière est prévu extra-muros, dans les lices de l'enceinte.

Mais cet état en recouvre un autre, antérieur, qui dans cette partic présentait un aspect monumental, avec des tombeaux de pierre. Les travaux se sont limités à une surface irrégulière, large de 6 mètres du nord au sud, longue de 8 mètres.

A l'intérieur, quatre tombeaux ont été découverts. Le sol, au moment de l'usage de la nécropole était à $2^{\mathrm{m}} 70$ sous le niveau du jardin, soit à un peu plus de $2^{\mathrm{m}} 50$ au-dessus du sol actuel de la salle basse de l'aula (fig. 54). Celte altitude est-elle due à l'accumulation des sépultures, ou à une remontée du sol naturel dans lequel cette salle serait taillée en encoche? Il n'était pas possible de faire une véritable fouille, mais simplement de constater l'état de ce qui allait être détruit. Les suls inférieurs n'étant pas menacés, ils n'ont donc pas été explorés.

Les quatre tombeaux étaient orientés (fig. 76 et 77 ). $\Lambda$ u sud se trouvait un caveau ( $\left.n^{\circ} 4\right)$. Les dalles verticales de l'encadrement avaient $0^{\mathrm{m}} 57$ de hauteur. Elles reposaient sur un socle chanfreiné. L'intérieur était aménagé en pourrissoir : deux dalles transversales étaient destinées à soutenir le cercueil, les os tombant au fond du caveau au fur et à mesure de la décomposition. De fait, s'y trouvait un amoncellement d'ossements, sans connexion anatomique, qui n'a pas été fouillé. Il est probable aussi que de tels caveaux ont servi d'ossuaire au moment de l'abandon du cimetière. La dalle de couverture de celui-ci était encore en place avant son enlèvement par la pelle mécanique. Au sud-est, engagé sous la coupe de terre, un tombeau ( $\mathrm{n}^{\circ}$ 2) n'a pas été ouvert. Il portait encore une belle dalle de couverture monolithe anépigraphe à l'arête inférieurt chanfreinée. La pierre de socle était aussi ornée d'un simple chanfrein. Le tombeau s'élevait de $0^{\mathrm{m}} 60$ au-dessus du sol. C'était peut-être comme le précédent, un caveau. Un autre caveau se trouvait au nord-ouest ( $\left.n^{\circ} 1\right)$. Scul le socle était encore en place, portant les traces d'arrachement des dalles verticales disparues; il était orné d'un tore surmonté d'un congé; ce monument avait servi d'ossuaire. La dernière tombe $\left(n^{0} 3\right)$ était une sépulture individuelle dont il restait un encadrement en deux assises formant socle, émergeant du sol. L'arête supérieure de l'assise la plus basse était seulement chanfreinée. L'assise superposée s'ornait d'un bandeau, d'un tore et d'un congé. La combinaison de toutes ces moulurations produisait un effet riche. Au centre de l'encadrement se trouvait un blocage qui avait dû être dissimulé par une dalle disparue. Deux lits superposés correspondaient aux deux assises moulurées. L'ensemble était posé sur une couche de mortier débordant largement sur le sol alentour. Au-dessous se trouvaient encore deux lits de pierre en radier, séparés par une couche de terre. La tombe proprement dite était recouverte de trois grandes lauses posées à la cote 203,20, maçonnées entre elles, et liées aux dalles levées formant les flancs d'un colfrage. Celui-ci était de forme anthropomorphe avec une courte cavité pour la tête. Les flancs étaient constitués chacun de trois dalles posées de chant. Il était profond de $0^{\mathrm{m}} 35$, long de $1^{\mathrm{m}} 80$ et contenait un corps d'adulte superposé à un squelette remanié.

Aucun matériel n'a permis de dater cette tombe que l'on ne saurait faire remonter à très haute époque. Compte tenu du contexte historique connu, elle peut avoir été creusée dans la première moitié du xvil ${ }^{\mathrm{e}}$ siècle, ou un peu avant. Mais, mise à part l'élévation au-dessus du sol, une telle sépulture présente peu de différences avec les tombes médiévales de qualité, que ce soit par le plan du coffrage, la couverture de lauses, la superposition de plusieurs niveaux de radicr, et enfin la réutilisation inattendue. 
Dans cette nécropole se trouvaient naturellement des remplois antiques. L'un d'eux était un long chaperon de mur en pierre froide, d'assez bonnes dimensions pour avoir servi de couverture à une inhumation ( $\left.n^{\circ} 5\right)$. Il a ćté retrouvé au sudest du sondage, sans rapport avec aucune tombe. Sa place, dans un endroit de la nécropole, à còté d'autres sépultures au couvercle arraché, est significatif des violations qui ont précédé l'abandon de ce niveau, et le comblement destiné à dissimuler des tombeaux sans doute trop denses, avant que de nouveaux défunts, plus pauvres, ne soient inhumés au même endroit.

Pour marginales qu'elles soient par rapport à notre sujet, il nous fallait signaler aussi ces tombes que la fouille a révélées; elles témoignent d'ailleurs à leur façon de la continuité de l'occupation humaine du secteur, en s'inscrivant à la suite de la tombe sous tuiles foullée à l'est de l'insula II et de l'inhumation placée sous la tour du palais archiépiscopal. Mais l'essentiel est évidemment ailleurs, plus au sud, dans la Cour elle-même dont la fouille a permis de connaître les transformations successives - et c'est là sans nul doute son principal acquis.

Nous avons trop insisté dans le corps de cet article sur nos incertitudes et nos hésitations pour présenter en conclusion une synthèse achevée. Pourtant, la confrontation avec les fouilles voisines, le recours aux représentations graphiques, aux sources documentaires anciennes, à l'étude des élévations conservées qui confortent également les résultats de la recherche archéologique proprement dite, bref les accuis de tous ces éclairages que nous avons recherchés et multipliés au risque de dérouter (et lasser) le lecteur nous conduisent à la conviction que c'est bien autour de la Cour que s'est joué le devenir du site.

De ce point de vue, la fouille de l'Archevêché apporte une nouvelle confirmation du conservatisme

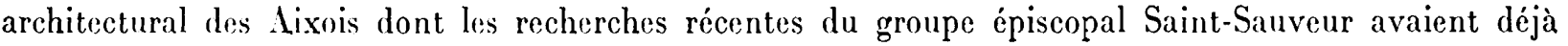
fourni un exemple marquant; mais tandis qu'à la cathédrale, les monuments chrétiens ont directement été fondés sur un grand édifice public romain, puis rebâtis sur un même plan à époque romane avant que les transformations de l'époque gothique et de l'âge classique ne leur donnent leur aspect définitif, il en est allé un peu différemment à l'Archevêché où l'évolution a été peut-être plus précoce et sûrement plus anple et où elle a consisté alıssi à transformer en édifice public une habitation privée à l'origine.

Tout a été commandé en effet par le choix qu'avait fait un architecte romain d'organiser une insula autour d'une cour d'une centaine de mètres carrés à peine : un espace trop restreint pour l'Antiquité tardive qui en repoussa les limites au moment où le monument passait (peut-être) déjà aux mains de l'Église ; trop vaste pour le premier Moyen Age puisque la cour a pu être divisée avant l'époque romane entre chanoines à l'ouest et évêtque à l'est. Dans le domaine archiépiscopal en tout cas - le seul qu'il nous soit donné d'entrevoir, la cour a sûrement gagné ensuite en importance et en monumentalité, au fil d'extensions successives qui traduisent l'affirmation d'un pouvoir ecclésiastique toujours soucieux de transformer un palais sans cesse remodelé à son goût et à son image.

Il peut être piquant de constater d'ailleurs que l'ultime transformation du palais, en 1985, s'inscrit dans le droit fil des interventions archiépiscopales : en repoussant jusqu'à l'impasse des Eyguesiers la façade des écuries de Charles de Villeneuve et en en faisant un simple fond de scène, la ville d'Aix-enProvence et la Direction du Festival ont atteint une limite désormais difficilement franchissable, portant du coup l'ensemble de la cour à une superficie de plus de mille mètres carrés. Én l'occurrence, il s'est agi d'ailleurs moins d'une transformation que d'un simple agrandissement puisque le mur sud a été démonté, puis remonté (et l'aile ouest prolongée de quelques mètres par une élévation postiche).

Hormis les gradins (démontables) du Théâtre du Festival, le $\mathrm{xx}^{\mathrm{e}}$ siècle n'a donc rien ajouté au décor du xvir ${ }^{\mathrm{e}}$ siècle; il a seulement changé les"proportions de l'ensemble et permis une fouille sur le site, qui révèle très largement la genèse d'un monument jusqu'alors décidément mal connu (mais non méconnu) (99). Faut-il l'en louer ou l'en blâmer? Aux lecteurs du Bulletin monumental d'en juger désormais. 


\section{ANNEXE I}

\section{Numismatique, par J.-L. Charlet}

Les monnaies sont classées par ordre chronologique; quand une identification précise est possible, leur description est suivie d'un renvoi à un ouvrage ou catalogue de référence.

\section{ANTIQuité}

Les monnaics issues des couches médiévales sont signalées par la mention "c. méd. " entre parenthèses; pour les monnaies trouvées dans des niveaux antiques, nous avons en revanche indiqué l'espace (cf. fig. 2)et la couche (cf. la nomenclature de la n. 18, p. 000) auxquels elles appartiennent.

1) Gaule. Volques Arécomiques. (c. méd.)

Tête de Diane à droite (volca $\mathrm{E}$ )

$\mathrm{R} /$ (Demos debout à gauche, une palme AREC)

Muret 2662 à 2683 (La Tour pl. VI, 2677)

2) Varseille. Petit bronze au taureau. (c. méd.)

Tête d'Apollon lauré à gauche (décentrée); derrière, $\overrightarrow{I_{A} I}$

R/MALEA (IITLN), taureau cornupète à droite

Brenot Cat. série 6, 23

3) Marseille. Petit bronze au taureau. (c. méd.)

Tête d'Apollon lauré à droite; devant, $\mathrm{N}$

R/MA MEA AT?, taureau cornupète à droile

Brenot Cat. série 9, 38

4) Marseille. Petit bronze au caducée (apris 19 avant J.-C.) (c. méd.)

Tête de Minerve casquée à droite

$\mathrm{R} /$ Caducie aile, $\Pi$ dans le champ

Brenot Cat. groupe V; Muret 2027

5) Rome. As posthume d'Auguste, frappé sous Tibère (après 22) (espace C 2, c. 8 c)

divgs avgrstrs pater tête (radiée) à gauche

R/ Providen(T) autel entre s c

R. I. C., I (p. 95), n ${ }^{\circ} 6$

6) Rome. As posthume d'Auguste, frappé sous Tibère (après 22) (c. méd.) Identique au précédent.

7) Rome. As de Germanicus frappé sous Claude (41-5/1) (troué) (espace C2, c. 8 c)

Germanicys Caesar Ti AVG F Divi avg $N$ tête nue à droite

R / (TI CLAVDIVS) CAESAR AVG GERM PM TRP IMP PP, au centre S C

R. I. C., I (p. 132), no 8 '́

8) Rome. Restitution par Titus d'un sesterce au nom de Divus Augustus frappé sous Tibère (79-81) (c. méd.) Div(vs avgrs)Tvs Pater, Auguste assis à gauche tenant une patère R/ T CAES (DIVI VESPI F AVG P M) TR P cos (VII), dans le champ sc (REST) R. I. C., II (p. 141), no 188

9) Rome. As de Domitien (85) (espace P3, c. 8 e)

IMP CAES DOMITIAN AVG GERM $\cos \mathrm{X}_{\mathfrak{z}}$, buste lauré à droite avec égide $\mathrm{R} /$ Moneta avgust sc, la Monnaje debout à gauche tenant une balance et une corne d'abondance R. I. C., II (p. 188), $\mathrm{n}^{\circ} 270$

10) Rome. As de Trajan (112-114) (espace C3, c. 8 c)

imp caes Nervae trainNo avg ger daC pM tRP ( $\cos$ vi PP), tête laurée à droite R/ SPQR OPTIMO PR(INCIPI) Sc, Victoire avançant à gauche tenant une branche et une palme R. I. C., II (p. 286), no 597

(11) Rome. Sesterce d'IIadrien (132-134) (très usé) (espace D3, c. 8 a)

Buste lauré à droite $\mathbf{R} /$ Galère

R. I. C., II (p. 431), no 706 (d?) 
12) Rome. Dupondius d'Iladrien (134-138) (espace C2, c. 8 c)

hadrianve avg cos irr pp, buste nu drapé à droite

R/ DaCra sc, Dacie assise à gauche sur un rocher, tenant un vexillum dans la main droite et une épée courbe dans la main gauche

R. I. C., II, $\mathrm{n}^{0} 580$ (b)

13) Rome. As d'Iladrien (13/1-138) (espace C2, c. 8 c)

(Hadrianvs) avg cos (III PP), têle laurée à droite

R/ AE(Qvit) AS AvG Sc, Équité debout à gauche

R. I. C., II, no $795 \mathrm{~d}$

14) Rome. Dupondius de Sabine, épouse d'Hadrien (c. méd.)

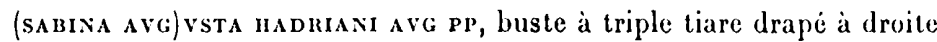

R/ sc Cérès assise à gauche sur un panier, tenant un épi et une torche

R. I. C., II (Hadrien), no 1023

15) Rome. As d'Antonin le Pieux (139) (espace (33, c. 6)

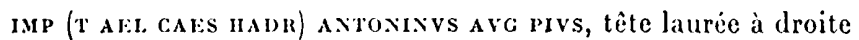

$\mathbf{R} /(\mathbf{P M r n}) \mathbf{P} \cos \mathrm{I}(\mathrm{I})$ sc, Paix debout a gauche tenant une branche et une corne d'abondance

R. I. C., III, n ${ }^{0} 532$

16) Rome. Sesterce d'Antonin le pieux (155-156?) (c. méd.)

antoninvs avg pr-vs pp imp ir, tête lauréc à droite

$\mathrm{R} / \ldots \times \operatorname{xix}(?) \ldots$, laix debout à gauche (?)

R. I. C., III, no 945 (?)

17) Rome. As posthume de Faustine $I$ (141-161) (espace C3, c. $8 \mathrm{c}_{i}$

Diva (FAvst) ina, buste à droite

R/ AEten-Nitas sc, Faustine assise à gauche sur le globe, tenant un sceptre dans la main gauche et levant la droito R. I. C., III (Antonin), $\mathrm{n}^{0} 1159 \mathrm{a}$

18) Rome. As posthume de Faustine I (après 1'1) (espace P', hors stratigraphie)

Diva Favstixa, buste à droite

$\mathrm{r} /(\mathrm{AvGv}) \mathrm{sT}(\mathrm{A}) \mathrm{Sc}$, l'Éternité s'avançant à gauche, tenant une torche

R. I. C., III (Antonin), no 1183 (?)

19) Rome. As de laustine II frappé sous Antonin (146-161) (espace J3, c. 8)

Favstixa avg - pul ave Fri, huste drapé à droite

$\mathrm{n} / \mathrm{w}$ wo sc, Junon deboul à gauche, tenant une patère et un sceptre

R. I. C., III (Antonin), no 1398

20) Rome. Dupondius de Marc-Aurèle (166) (espace P7, c. 8 a/b)

m. avrel antoninvs avg arm parth max, tête radiée à droite

R/ TR POT (xx) IMP III cos III Sc, Rome assise à gauche tenant le Palladium et une lance; derrière elle, un bouclier R. I. C., III, no 937

21) Rome. As de Faustine II frappé sous .Iarc-Aurèle (après 161) (c. méd.)

Favstiva avgrsta, buste à droite

R/ I/vNONI REGINA)E SC, Junon debout à gauche tenant une patere et un sceptre; à ses pieds, un paon

R. I. C., III (Marc-Aurèle), no 1652

22) Rome. As de Commode (178) (espace II, c. 6)

r. Avnel Com - Modvs avG, buste lauré à droite

R/ vota PVBlica thP II(I) IMP II cos Pp Sc, Commode debout à gauche sacrifiant sur un trépied

R. I. C., III, $\mathrm{n}^{0} 1598$

23) Rome. Antoninien de Trajan Dèce (2/49-251) (c. mèd.)

IMP C M Q TRAIANvS DECivs Avg, buste radié, drapé et cuirassé à droite

R/ Gevivs (exerc iL) Lvriciavi, Génie à gauche portant un polos sur la tête, tenant une patère et une corne d'abondance; à droite, une enseigne

R. I. C., IV, $3, \mathrm{n}^{\circ} 16 \mathrm{c}$

24) Cyzique neoantoninianus Dioclétien (295-299) (c. méd.)

imp C (c) Val diocletiaxivs PF Avg, buste radié, drapé et cuirassé à droite

R/ CoNconda (MI)- LITvM KT, le prince debout à droite recevant de Jupiter debout à gauche une Victoire sur un globe

R. I. C., VI, $\mathrm{n}^{0} 15$ a 
25) Constance II (351-361) (c. méd.)

DN coxstas- ..., buste diadémé drapé à droite

$\mathrm{R}$ / type (FEL TEMP hFPARATio), soldat avançant à gauche, transperçant de sa lance un cavalier tombé As 3 , marque d'atelier illisible

26) Arles. Valentinien I (367-37j) (c. mèd.)

D. valextrixi-axvs pF avg, buste diadémé perlé, drapé et cuirassé à droite

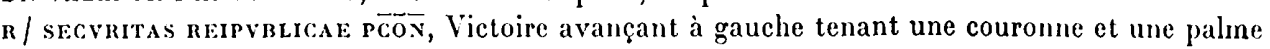

R. I. C., IX, $\mathrm{n}^{0} 17 \mathrm{a}$

2) Arles. Gratien (378-383) (c. méd.)

(DN Gn)A(TrA)-Nvs PF (A) v(G), buste diadémé perlé, drapé et cuirassé à droite

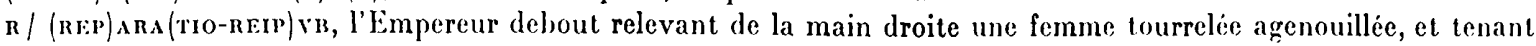
une victoire sur un globe de la main gauche $\overline{(s ?)} \overline{\mathrm{s}} \overline{\mathrm{N}}$

R. I. C., IX, no 20 a

28) Arles. lavius Victor (387-388) (c. med.)

DX Fi vic-Ton pF avG, buste à diadème perlé, drapé et cuirassé à droite

n/ spes no-ma-xonvs, porte de camp surmontée d'une étoile pón

R. I. C., IX, $\mathrm{n}^{0} 29 \mathrm{~b}$

29) Petit bronze du Bas-Empire très usé (c. mèd.)

A / . . cox... : famille constantinienne? diam. $12 \mathrm{~mm}$

30) Petit bronze antique non identifiable (c. méd.)

$\mathrm{A} / \ldots \ldots \mathrm{s} \mathrm{n} /$ grénetis ... diam. $11 \mathrm{~mm}$ (épaisseur $2 \mathrm{~mm}$ )

31) Petit bronze du Bas-Empire non identifiable (c. méd.)

diam. $19 \mathrm{~mm}$.

Moyen Age er Trmps Modernes

Localisation d'après les espaces indiqués sur les figures $35,36,45,49$ et 71

Monnaies.

32) Provence. Alphonse I et II d'Aragon (1167-1196-1209) (Esp. III b c. 3).

Royal coronat de Marseille $1^{\text {er }}$ type Rolland $11\left(\right.$ xir $\left.^{\mathrm{e}}-\mathrm{xini}^{\mathrm{e}}\right)$.

33) Identique au 32 (N-O cour h. s.).

34) Provence. Alphonse I et II d'Aragon (Esp. VIII c. 4).

Fragment d'obole de royal coronat $1^{\mathrm{er}}$ type Rolland $12\left(\mathrm{xII}^{\mathrm{e}}-\mathrm{xIII}^{\mathrm{e}}\right)$.

35) Provence. Alphonse II d'Aragon (xin ${ }^{\mathrm{e}}$ (Esp. V a c. 4).

Fragments de royal coronat $3^{\mathrm{e}}$ type, point creux après goxe Rolland $15 \mathrm{a}$.

36) Provence. Forcalquier. Guillaume $V$ de Sabran (1209-1220) (Esp. VIII c. 4). Denier guillermin Rolland $t_{t}$

37) Languedoc. Melgueil. (xine ${ }^{\text {e) }}$ (Esp. VIII c. 3).

Denier à légende dégénérée PA 3843 (pl. LXXXV, no 17).

38) Identique au 37 , mais cassé (Esp. V c silo 1).

39) Languedoc. Melgueil (xine?) (Lsp. V c silo 2).

Obole à légende dégénérée PA 38'1́ (pl. LXXXV, no 18).

40) Identique au $n^{0} 39$ (Lisp. V c silo 2).

11) Maine. Denier immobilisé au type d'Herbert ( $\mathrm{xuI}^{\mathrm{e}}$ ?) (Esp. VIII c. 3). PA 156' (pl. XXX, no 2)

12) I.e Puy. Fragment de denier de type dégénéré ef. PA, pl. XLIX, nº 7 (Esp. VIII silo 4).

43) Orange. Raymond III (1335-13\%0) (Esp. VIII a c. 3).

Denier PA 4485 (ou $4_{4} 486$, sans croisettes). 
44) Royaume de Castille et de Leon. Fernando IV (1295-1312). Burgos.

Cf. IIeiss, t. 1, pl. 6, no 7 ; p. $48, n^{0} 7$ (Esp. VII c. 3).

$\dagger$ movira castriff, château castillan dans un polylobe; dessous, B.

n / : ET r.giosis, lion a gauche dans un polylobe.

45) France. Charles VIII, Karolus ou dizain (1/88-1498) (Esp. VIII d c. 2).

Atelier non identifiable. Lafaurie 568.

46) France. La Ligue, double tournois au nom d'IIenri III, 1592. Lafaurie 1013. (Esp. II h. s.).

47) France. Double tournois très usé; IIenri IV? (Esp. II h. s.).

48) Avignon. Fragment de patac pontifical $x_{v I^{e}}{ }^{-} \operatorname{VvII}^{\mathrm{e}}$ (Esp. IX c. 1-2).

49) France. Louis XIII, double tournois au buste lauré et drapé (i1) 11 (1638) (h. s...

50) Double tournois très usé non identifiable, $\mathrm{xvir}^{\mathrm{e}}$ (Esp. III c. 1).

51) Petit billon non identifiable; diam. $11 \mathrm{~mm}$ (Esp. V c c. 4).

52) Petits fragments de monnaie non identifiable (lisp. VIII c. 3).

53) Fragments de deux monnaies pulvérisćes (Esp. IX c. 1-2).

54) Plaisance $\left(\mathrm{x}_{11}{ }^{\mathrm{e}}-\mathrm{x}_{11} \mathrm{e}^{\mathrm{e}}\right.$ siècles) (sous la cheminée, c. 4 ).

Obole, type immobilisé (identification M. Bompaire).

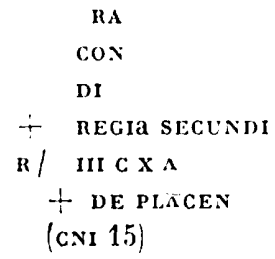

Méreaux.

55) Plomb médiéval (Esp. VI a c. 3).

56) Plomb médiéval (Isp. VI b c. 3!

57) Jeton médiéval. Italie ou Paris (hanquiers lombards, probablement Biccherma de Sienne) (Esp. VII c. 3j.

B entouré de 15 besants

$\mathrm{n} /$ brouillé, 18 besants laiton, $23 \mathrm{~mm}$.

Piton, no 82 , Feuardent 4969 pour le droit (voir ('. Démians d'Archimbaud, Rougiers..., p. 264, no 120 , et photo, p. $\left.274, n^{\circ} 120\right)$.

Répartition chronologique :

sur les 31 monnaies antiques, dont 28 identifices, on relève :

- 4 monnaies du $\mathrm{r}^{\mathrm{er}}$ siècle avant J.-C.

- 5 monnaies du ${ }^{\text {er }}$ siècle après J.-C.

-.- 13 monnaics du II $^{\mathrm{e}}$ siècle après J.-C.

- 2 monnaies du ni $^{\mathrm{e}}$ siècle après J.-C.

... \& monnaies (plus, vraiscmblablement, deux autres non identifiées) du rve siècle (seconde moitiè).

Les quatre monnaies préromaines sont méridionales ( 3 massaliètes et une des Volques $A$ récomiques). Du point de vue quantitatif, le monnavage le plus important est celui du ${ }_{{ }_{1}}{ }^{\mathrm{c}}$ siècle après Jésus-Christ, essenticllement d'Hadrien à Cummode. Toutes les monnaies du $x^{\mathrm{e}}$ siècle dont l'atelier est identifiable proviennent d'Arles; on notera le petit lironze de Flavius Victor. on relève:

Sur les 19 monnaies médiévales (plus deux plombs et un jeton) ou modernes au moins partiellement identifiables,

- 11 monnaies des $\mathrm{xII}^{\mathrm{e}}-\mathrm{xIII}^{\mathrm{e}}$ siècles, dont 5 provençales et 4 melgoriennes.

- 2 monnaies du xive siècle (Orange et Espagne).

- 1 royale française de la fin du $x^{e}$ siècle.

- 5 monnaies de la fin du $\mathrm{xvI}^{\mathrm{e}}$ ou du début du $\mathrm{xvir}^{\mathrm{e}}$ siècle, dont 3 ou 4 françaises et une monnaie d'Avignon. 


\section{ANNEXE II}

Marques relevées sen le matériei. cémamique recueilli en stratigraphe

1) Marques sur sigillées du Sud de la Gaule (La Graufesenque) (fig. 78).

- no 16766, Esp. P5; contexte : flavien; sur fond de plat : vapvsones.F : pour vapvso cf. Osw. : Tibère-Néron.

- $\mathrm{n}^{0} \quad 10 \frac{1}{4}$, Esp. K; contexte : milieu $\mathrm{Ir}^{\mathrm{e}}$ siècle; sur fond de Drag. $33: \mathrm{Fv}$ [; non lue.

- $\mathrm{n}^{\mathrm{o}} 107$, Esp. $\mathrm{K}$; contexte : milieu $\mathrm{II}^{\mathrm{e}}$ siècle ; sur fond de Drag. 33 : of.v [; non lue.

- $\mathrm{n}^{\circ} 12382$, Esp. L; contexte : Antiquité tardive; sur fond d'assictte ombiliqué : șe[..]çve; non lue.

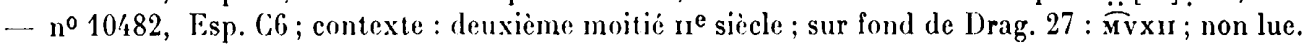
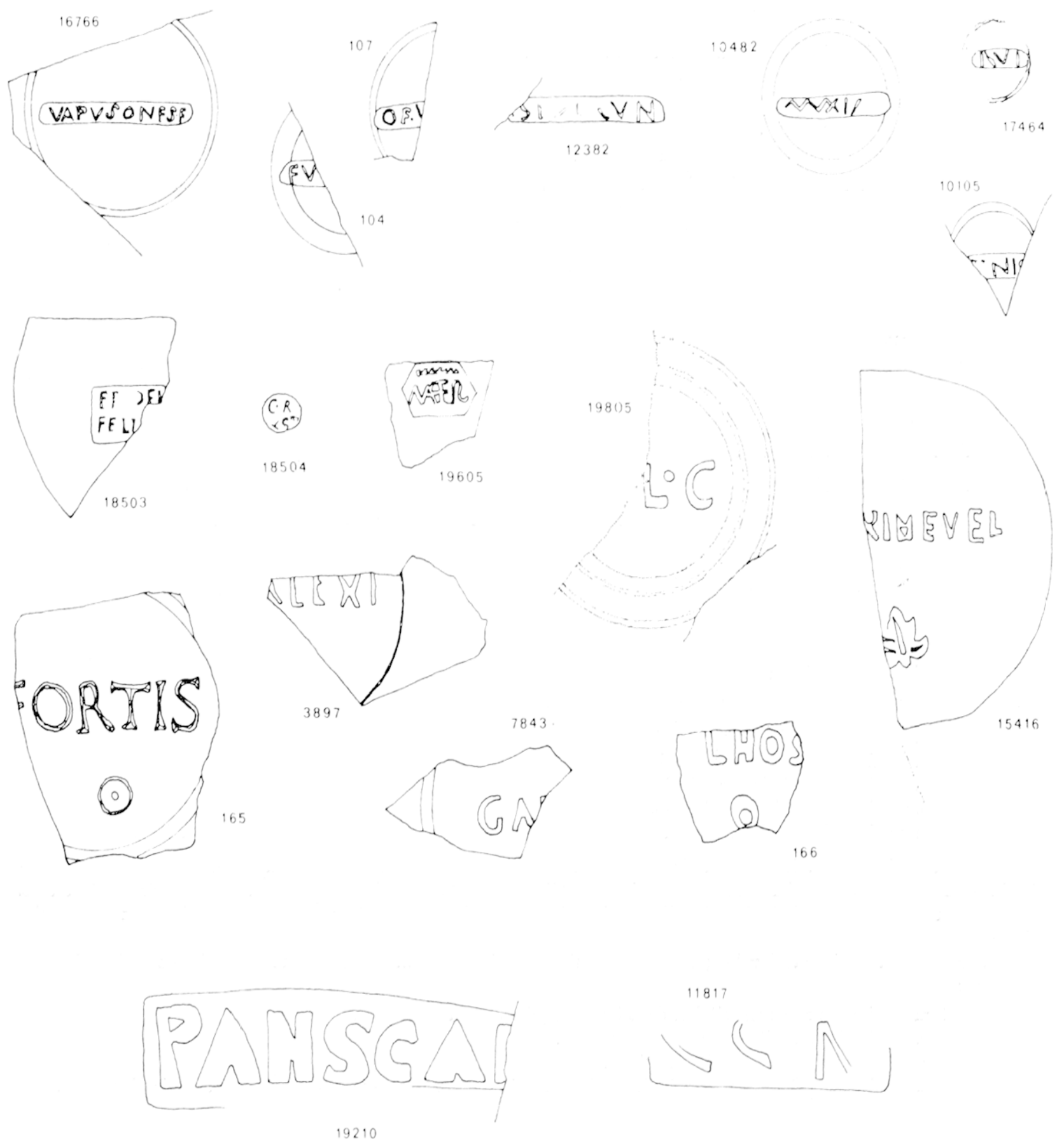

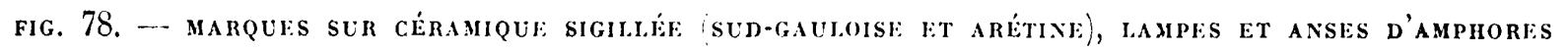



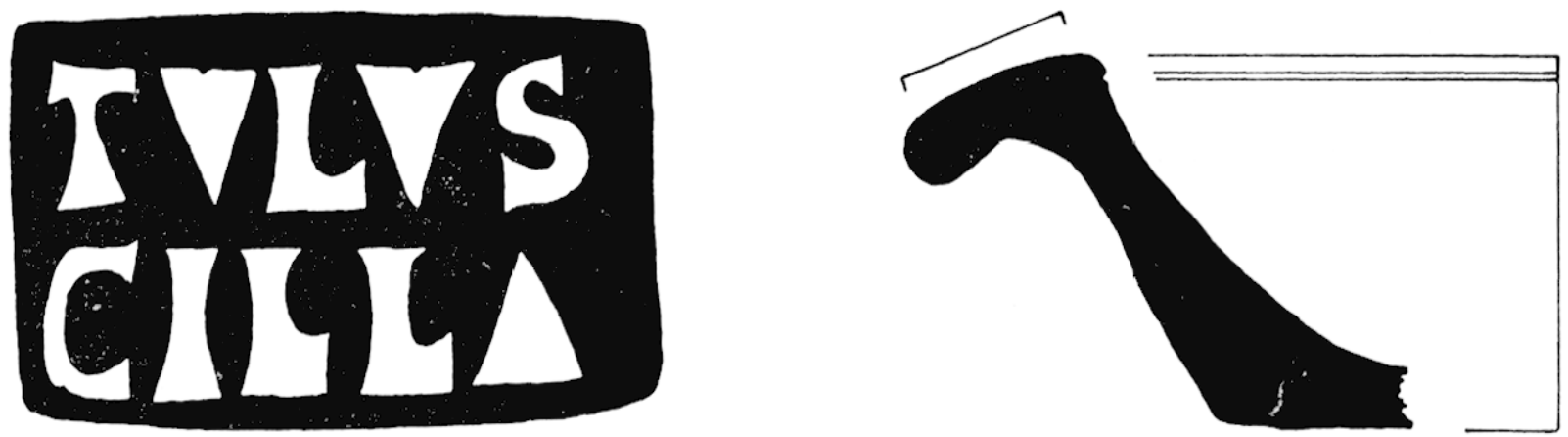

15919
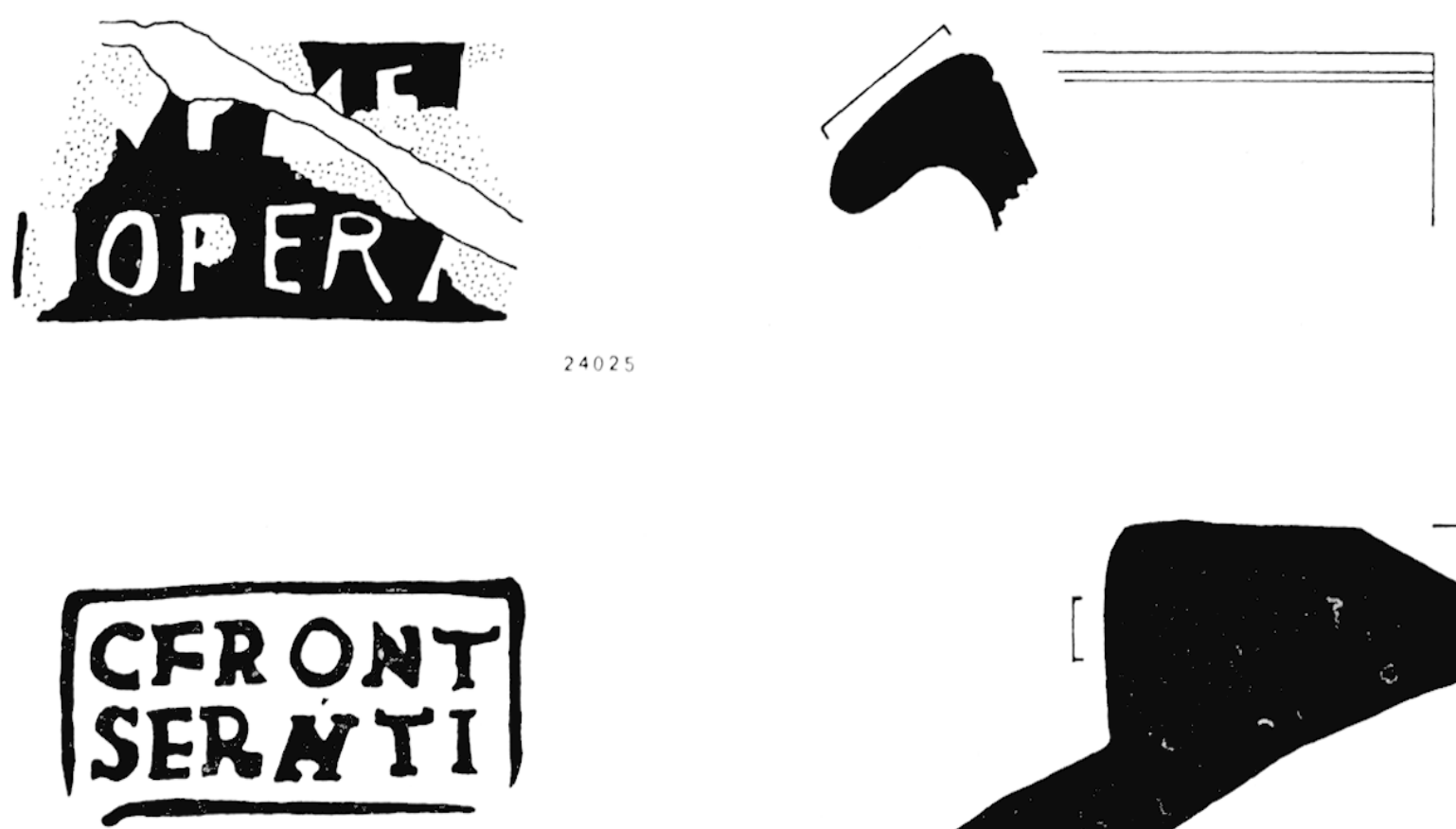

8605

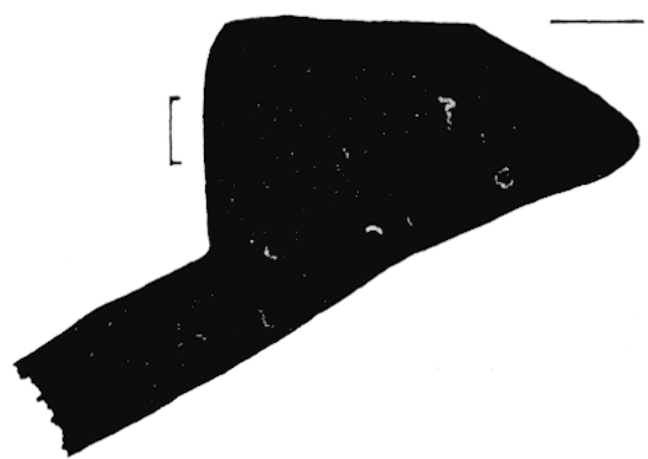

FIG. 79. - MARQURS SUR MORTIERS FT SUR DOLIUM

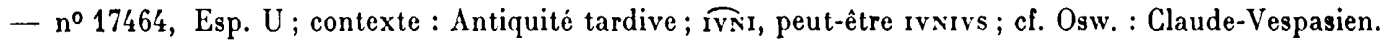

- no 10105, Esp. C6 ; contexte : Antiquité tardive ; ..]Nis ; non lue.

2) Marques sur arétine (fig. 78).

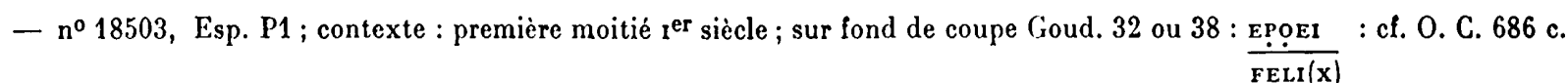

- no 18504, Esp. P1; contexte : première moitié ${ }^{\text {er }}$ siècle ; sur fond de coupe : crest = crest(vs), cf. O. C. 425.

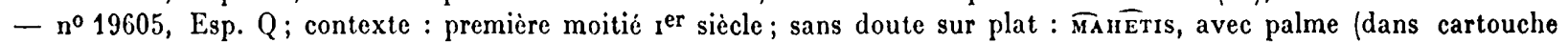
hexagonal inédit); pour manEs, cf. O. C. 169. 
3) Marques sur lampes (fig. 78).

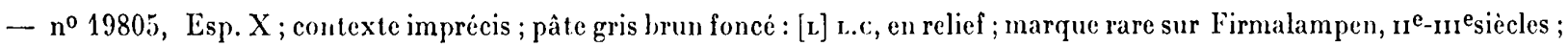
importation d'Italie du Nord.

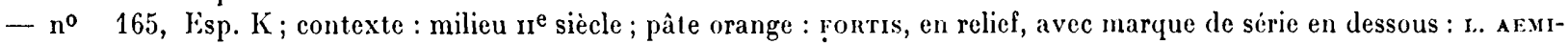
uivs fontrs; atelier en Italie du Nord.

- $n^{0} \quad 166$, lisp. $\mathrm{K}$; contexte : milieu $n^{\mathfrak{e}}$ siècle ; pâte jaune clair, engobe brun : Lros[, en creux, avec marque de série en dessous : ivcrvs hosidrvs crispvs (milieu $\mathrm{I}^{\mathrm{er}}{ }_{-\mathrm{II}}^{\mathrm{e}}$ siècles), atelier en pays voconce.

- $\mathrm{n}^{0} 15416$, Esp. $C_{4}$; contexte $: \mathrm{II}^{\mathrm{e}}$ siècle; pâte jaune clair, engobe orange : ]nimever., cn creux, avec marque de série en dessous, sans doute une des oflicines de la figlina (L.) Farricia; importation vraisemblable d'Italie.

- $\mathrm{n}^{0} 3897$, Esp. C2 ; contexte : deuxième moitié $\mathrm{II}^{\mathrm{e}}$ siècle; pâte jaune orange, engobe orange : ] 1 r.Fxr, en creux : (Cai) IVNI ALEXI; importation africaine possible.

- no 78'3, Esp. C3; contexte : milieu ri ${ }^{\mathrm{e}}$ siècle ; pâte jaune clair, engobe brun orange : ca[, en creux : canını (première moitié ${ }_{1}{ }^{\mathrm{e}}$ siècle sur lampe à bec rond), importation africaine.

4) Marques sur anses d'amphores (fig. 78).

- $n^{\circ}$ 19210, Esp. Q; contexte : deuxième moitié II $^{\mathrm{e}}$ siècle; sur Dressel 20 : passca!l, en relief, sans doute de la figlina scarkessia; Callender 1576.

- $\mathrm{n}^{\circ}$ 11817, Esp. C6 ; contexte imprécis : $\mathrm{I}^{\mathrm{e}}$ siècle?; sur Dressel $20:$ ça , en relief ; ccru plutôt que cc:xs (il ne semble pas y avoir la place pour le s); Callender 276.

5) Marques sur mortiers (fig. 79).

- $\mathrm{n}^{0} 15919$, Esp. C' $\overline{\text { CII.t.A }}$

- no 24025 , Esp. C2; contexte imprécis; ]p̣! [, en relief, non identifiée.

$$
\text { ]OPERạ }
$$

6) Marque sur dolium (fig. 79).

- $n^{\circ} 8605$, Esp. C3; contexte milieu II $^{\mathrm{e}}$ siècle; c rnoNT, en creux : sans doute C(ai) rrovr(ini) sfr(gia tribu) avitr. $\overline{\operatorname{sen} \widehat{A V T I}}$

(1) L'opération, préparce avec le plein accord de M. J.-P. de Peretti della Rocca, maire d'Aix-en-P'rovence, sur l'initiative de M. M. Gauther, directeur des Antiquities Historiques de la région Provence-Alpes-Cìte d'Azur, a duré du 26 septembre 1984 au 8 janvier 1985 ; elle a fait l'objet d'un financement conjoint de la ville d'Aix et du llinistire de la Culture (Sous-Direction de l'Archíologie) qui a couvert la fouille, son exploitation scientifique et la préparation de sa publication. Les responsables de la fouille ont été les signatiaires du présent article, assistés de vacataires, F. Bertrand, B. de Luca, P. Reynaud et I. Sciallano; de fouilleurs bénévoles, C. Blanc, II. Griesheimer, X. Rohmann et d'une cinquantaine d'ouvriers, parmi lesquels des fouilleurs : I. Bay; (i. Jucout, M. Jabéssouille, P. de I.uca, B. Ferraro, R. Pasquini et S. Schindler. Ont contribué à l'exploitation scientifique des résultats divers services de l'Lniversité de Provence (Aix-Marseille I) et du C.X.R.S. (Centre Camille Jullian, G. I. S. "Recherches sur l'aire méditerranéenne ", Laboratoire d'Archéologie Médiévale Méditerranéenne, Institut de Recherche pour l'Architecture Antique, Bureau d'Aix-en-Provence) ainsi que des chercheurs amis : J.-I. Gharlet, (G. Démians d'Archimbaud, P.-A. Février, J. Gascou, B. I.iou, J. et Y. Rigoir, J., Vallauri. D. Rouvier (I. A. II. M.) et I. Vincent (C. C. J.) ont assuré la dactylographie du manuscrit.

(2) J'équipe du Bureau d'Aix-en-P'rovence de l'I. R. A. A. : P. Varène, architecte, J. Bigot, ingénieur' ;.--I. Gassend, architecte et J.-M. Joulain, technicien, a également bénéficié du concours de G. Mlagdinier, architecte. Les relevés originaux sont conservés au Bureau d'Aix-en-Provence de l'I. R. A. A.; les 1.500 négatifs noir et blanc du chantier sont archivés à la photothèque du C. C. J. ; les objets recueillis par la fouille ont été déposés au Musée (iranet d'Aix-en-Provence.

(3) Car bien des éléments (notamment dans le quart nord-ouest de la Cour) ont été relevés sans qu'il ait été possible de les fouiller véritablement.

(4) Sud-ouest/nord-est en fait; pour éviter de trop alourdir la nomenclature quand nous aurons à indiquer des directions, nous avons choisi un nord conventionnel aligné sur le tracé des murs transversaux de l'insula I; tous les plans permettent cependant de connaître aussi le nord géographique.

(5) Indication approximative fournie par les cotes des ressauts de fondation des murs attribuables à l'état 1 (cf. fig. 4); noter que dans son état achevé, l'insula connaît de pareilles différences de cotes entre les pièces du secteur méridional et celles du nord.

(6) La banquette a été reconnue partout où nous avons pu mener la fouille à son terme, c'est-à-dire essentiellement en D1 et D3.

(7) I.e mur appuyé sur le flanc occidental de l'espace Q a cependant une apparence plus médiocre que le reste du mur 30 (cf. p. 208) mais à considérer le plan, peut-on douter qu'il s'agit bien pourtant d'un élément du mur de façade de l'insula achevée?

(8) Car le cardo fouillé dans la cathédrale Saint-Sauveur se compose d'une chaussće pavée large de $4_{4}^{\mathrm{m}}{ }_{4} 5$ et de deux trottoirs dallés larges de $1^{\mathrm{m}} 20$. 
(9) Sauf peut-être un bloc de $0^{\mathrm{m} 73} \times 0^{\mathrm{m} 79}$, épais de $0^{\mathrm{m}} \mathbf{1 0}$, qui a été retrouvé dans le comblement d'un silo médiéval; mais il est vrai que si l'hypothèse que nous présentons ici avec prudence doit être retenue, les éléments d'un éventuel dallage primitif auraient été très tôt récupérés.

(10) Sur les rues de líijus dans le seul secteur où elles ont étí largement reconnues -- c'est-à-dire au Clos de la Tour -, voir la thèse de II ${ }^{\mathrm{e}}$ cycle (dactylographice) de I. Rivet, Fouilles du Clos de la Tour à Fréjus (Var) : les rues, Aix-en-Provence, 1980.

(11) Sur les rues antiques repérées a $\lambda$ ix, voir en denier lien l'étude, précieuse par ses indications, mais discutable pour ses interprétations, de R. Ambard, Aix romaine, Aix-en-Provence, 198', notamment p. 85-117.

(12) La source unique en la matière est le mémoire d'A.-lE. (Gibelin, Lettre sur les tours antiques qu'on a démolies à Aix-en-Provence et sur les Antiquités qu'elles renfermaient, Aix-en-Provence, 1787; pour une interprétation de ses données, voir M. Clerc, Aquae Sextine, histoire d'Aix-en-Prowence dans l'Antiquilé, Aix-en-Provence, 1916 (reproduction anastatique, Marseille, 1973), p. 362, et R. Ambard, op. cit., p. 87-88 et 211-218.

(13) Ce qui ne va pas d'ailleurs sans difficultes car la porte d'Jlalie n'est nullement à l'alignement de l'autre portion du cardo maximus que l'historiographie aixoise place habituellement en façade de la cathédrale Saint-Sauveur (cf. fig. 1, $n^{\circ} 5 j$, ch supposant un changenent de direction a hauteur de la césure (médievalej entre bourg saint-Sauveur et ville comtale. Soter en revanche que la porte est dans l'axe du "boulevard" que nous proposerons d'ici peu de restituer à l'est du site de la Cour de l'Archerêche : hasard?' ou fait exprès?

(1'1) La rue de Celony et la rue du Bon-Pasteur relient en elfet le groupe cathédral (et donc le forum sous-jacent) a la seule portion de decumanus qui ait éte identifié à $\mathrm{Aix}$ (cf. fig. $1, \mathrm{n}^{0}$ / ) ; mais comme Je suggère suffisamment le plan, une telle jonction n'est possible qu'au prix d'un gauchissement non négligealle du tracé qui peut être aussi bien un héritage direct de l'Antiquite que le fruit de transformations médiévales... En l'état actuel de la recherche, quand la voie romaine est à près d'un kilomètre du bourg Saint-Saureur, il est impossible de restituer sûrement la topographie antique.

(15) Ce qui n'est pas le cas : rien n'évoque dans ce mur la construction régrulière des appareils liaisonnés à l'argile du premier état d'occupation des lieux et les blocs qui le constituent sont d'ailleurs plus petits que ceux des murs antiques du site. Comme le mur 116 ne joue aucun ròle daus les transformations médiévales du palais archiépiscopal, faut-il penser qu'il appartient à l'Antiquité tardive ou au haut Moyen Age? C'est possible, mais la fouille n'autorise aucune certitude en ce domaine.

(16) Voir une bonne présentation de l'ètat de la question dans R. Ambard, op. cit., p. 52-60 ; l'auteur refuse cependant de placer une limite urbaine a hauteur de la rue l'ierre-el- llarie-Curie par le hiais d'arguments qui n'emportent pas totalement l'adhésion : les mosaïques qui ont été retrouvées plus à l'est (notamment au ljocée Campra) peuvent appartenir à des habitations périphériques, non à des insulae urbaines...

(17) La prudence de la formulation s'explique parce qu'une linite urbaine n'est pas toujours matérialisée par un rempart; en attendant la publication des $A$ ctes du Colloque sur Les enceintes augustéennes de l'Occident romain (Nimes, 11)-11 octobre 195:5) dont les débats ont Jonguerrent insisté sur ce point, voir un exemple fourni par la recherche récente et volontairement choisi dans une toute autre région : R. Neiss, La structure urbaine de Reims anlique et son érolution du Ier au $I I I^{\mathrm{e}}$ siècle après $J . C^{-}$., dans Revue archéologique de Picardie, 3-4, 1984, p. 171-191.

(18) La somme des tessons recensés dans les différents tableaux placés dans le cours du texte est inférieure à ce nombre ; c'est que nous n'avons présenté dans ces tablcaux que les couches les plus significatives. I)'autre part, la nomenclature des couches a été harmoniséc pour l'Antiquité; c. $10: \mathrm{I}^{\text {er }}$ siècle (essentiellement ćpoque flavienne); c. $9:$ première moitié du ${ }_{11}{ }^{\mathrm{e}}$ siècle; c. $8:$ deuxième moitié du $\mathrm{n}^{\mathrm{e}}$ siècle ; c. $7: \mathrm{nn}^{\mathrm{e}}$ siècle; c. $6:$ Antiquité tardive et haut Mloyen $A g e$.

(19) Car les fouilles au-dessous du groupe cathédral voisin n'ont pas livré non plus de vestiges antérieurs au début de notre ère (cf. infra, n. 26); il est vrai cependant que tout le secteur occidental du burgus reste à découvrir (cf. fig. 1) : réservons l'avenir!

(20) Les menues différences de cote entre des points parfois assez voisins s'expliquent par des remaniements, toujours fréquents sur les chaussées.

(21) Noter qu'en P1 comme en C2 et C.3, les couches 10 traduisent le comblement de fosses profondes qui recèlent un matériel abondant : 890 des 1.151 tessons recueillis par la fouille de ces niveaux anciens (et sporadiquement reconnus).

(22) Les conditions d'observation sont médiocres à cause de l'importance des réoccupations et des récupérations médiévales qui ont affecté la pièce $K$ et les murs qui lui servent de limites : cf. p. 206.

(23) Si du moins les sols de l'insula agrandie sont restés à même cote que ceux (supposés) de l'insula primitive; cf. supra, p. 200 et n. 5.

(2') Car le béton du sol tardif de la pièce épouse exactement le tracé d'un mur qui a certainement été refait, comme le montre le coup de sabre oblique placé à proximité de sa liaison avec le mur 1 (cf. fig. 2) ; l'enduit peint que nous venons de décrire n'est d'ailleurs conservé sur le mur qu'au sud du coup de sabre.

(25) Lne étude plus serrée révèlerait d'autres indices possibles de transformations au sein d'un édifice qui fut sans doute sans cesse remanié (cf. pour une époque plus tardive, les sols en béton superposés de la pièce I, infra, p. 210!.

(26) Pour les fouilles du groupe cathédral, voir R. Guild, J. Guyon, L. Rivel, Recherches archéologiques dans le cloître Saint-Sauveur d'Aix-en-Provence - bilan de quatre campagnes de fouilles (19;6-1!9i9), dans Revue archéologique de Narbonnaise, 13, 1980, p. 115-169; Id., Les origines du baptistère de la cathédrale Saint-Sauveur - étude de topographie aixoise, ibid., 16,1983, p. 171-232; pour celles de la place des Nartyrs-de-la-Résistance, l'article d'A. Kauffimann, Cardo et place dallée à Aix-en-Provence - fouille de sauvetage sur la place des Martyrs-de-la-Résistance, asril 1:\$1, ibid., p. 233-2'16.

(27) Voir les reconstructions topographiques de R. Ambard, op. cit., $2^{\mathrm{e}}$ partie, passim et plan p. 86, que les présentes recherches de la Cour de l'Archevêché in firment très largement.

(28) Voir supra, p. 199, une présentation rapide des indices qui suggèrent que le mur nord de l'insula est fait d'éléments juxtaposés qui peuvent témoigner d'extensions progressives; d'autres indices de possibles transformations seront donnés infra. 
(29) Cf. Infra. Il est vrai que cette extension est marquée par la construction d'un portique sur la façade est de l'insula, non par l'aménagement de pièces couvertes comme ce fut peut-être le cas dans l'insula II.

(30) A propos du rempart, voir supra, p. 203 et n. 17; quant au decumanus nord, son existence parait suffisamment prouvée par la limite franche tracée par le mur $\$ 3$ (ou, du moins, par le négatif laissé par la récupération de ses matériaux) et par l'absence de tout vestige antique plus au nord; ajoutons qu'à Aix où les égouts du secteur sont tous à mettre en relation avec la voirie, l'examen du système de drainage du site plaide également en faveur de l'existence d'une rue à cet endroit : outre l'égout domestique de l'insula I qui se déverse vers le nord (cf. infra, p. 213), il faut signaler la présence d'une seconde canalisation, dirigée vers le sud cette fois, qui a été reconnue sommairement dans les fondations de l'aile nord du palais archićpiscopal, lors de la reprise en sous-œuvre du bâtiment; les deux égouts convergaient-ils vers la tranchée de direction est /ouest qui a été repérée, 2 mètres environ au sud du mur du palais? Il est impossible de l'affirmer, et l'on notera seulement (sans dissimuler la fragilité de l'argument) que si cette tranchée garde bien la trace d'un collecteur axial, les dimensions qu'il faudrait alors ı estituer au decumanus nord seraient fort modestes (5 à 6 mètres de large tout au plus).

(31) Pour permettre des comparaisons rapides et commodes, nous nous bornerons à renvoyer à l'étude classique de P.-A. Février, M. Fixot, Chr. Goudineau et V. Kruta, Histoire de la France urbaine, t. I, Paris, 1980, dans laquelle le lecteur trouvera, aux p. 248-25'4 une série de plans de villes à même échelle (à l'exccption du plan d'Arles, qui a fait l'objet d'une réduction erronée) : ce sera l'occasion de vérifier la modestie des îlots aixois, comparés aux insulae de la plupart des autres villes de Gaule (il est vrai que, dans bien des agglomérations, les dimensions des îlots sont restituées).

(32) Comme le revêtement de tuiles qui scelle nettement les couches sous-jacentes n'est pas present sur toute la surface du sol établi à la cote $203,35 \mathrm{~m}$., il reste théoriquement possible que des tessons du remblai sous-jacent aient migré dans la couche inférieure; la prudence nous commande de présenter ici cette hypothèse à laquelle nous ne croyons guère cependant.

(33) 816 fragments de vases, soit $92,2 \%$ des tessons de la couche 8 - et même $95,7 \%$ en ajoutant les 32 fragments de couvercles.

(3i) Pour se jeter dans le collecteur axial supposé du decumanus ‘cf. supra, n. 30)?

(35) $\Lambda$ insi qu'en témoignent les enduits peints conservés au-dessous du niveau des bétons, qui sont à metlre en relation avec un sol primitivement situé à un niveau inférieur (cf. fig. 12).

(36) Eucore des tessons de sigillée claire A, des formes (datantes) Lamb. $10 \mathrm{~A}$ et $10 \mathrm{~B}$, mais aussi un col d'amphore assimilable à la forme Gauloise $3\left(\mathrm{n}^{0} 1973\right.$, pâte ocre fortement micacée) et des couvercles en céramique commune à pâte claire (nos $2315,2320,2325)$ et, à nouveau, une cale de four.

(37) Et dont la couverture est également hypothétique : un toit à deux ou quatre pentes?

(38) Aire du Chapitre : fouilles de Rouard (1842) et de R. Ambard (1948), reprises en 1985, qui donneront lieu rapidement à une publication exhaustive; Jardin de Grassi : publication de F. Benoit, La maison à double péristyle du Jardin de Grassi, dans Gallia, 5, 1947, p. 98-122 ; École des Beaux-Arts : Informations archéologiques, dans Gallia, 35, 1977, p. 512; Pavillon Vendôme : cf. Forma orbis romani - Carte archéologique de la Gaule romaine, fasc. 5, Bouches-du-Rhône, Paris, 1936, p. 72, $\mathrm{n}^{0} 46$.

(39) Force est de nous en tenir aux renseignements fournis par l'étude du seul secteur médian du boulevard (?) car il nous a élé impossible de fouiller convenablement les zones situées plus au nord et plus au sud.

(40) Pour une présentation rapide de ces objets que nous ne pouvons qu'évoquer ici, nous renvoyons à notre plaquetle : M. Fixot, J. Guyon, J.-P. Pelletier, L. Rivet, La fouille de la Cour de l'Archevêché (sept. 1984-janv. 198:5), Documents d'archéologie aixoise, Aix-en-Provence, 1985.

(41) Un seul dé a pu être fouillé, en D4; mais le collage des deux èléments du mur en D2 peut être aussi à la hauteur d'un autre dé (?); à tout le moins tient-on là la preuve que, comme les murs 36 et 42 , le mur 51 est fait de segments juxtaposés.

(42) Car nous n'avons retrouvé aucune trace d'un sol en béton de tuileaux à l'ouest de ce mur, en P8.

(43) La fouille du remblai sous le bèton de l'espace $R$ a pourtant livré 183 tessons, dont les plus récents exemplaires datables sont postérieurs au milieu du $1^{\text {er }}$ siècle de notre ère; mais le reste de la fouille montre qu'à la fin du $1^{\text {er }}$ siécle let probablement plus tard encore), tout l'espace à l'est des insulae était sûrement ouvert à la circulation...

(44) Si l'interprétation est fondée, la restitution que nous avons proposée pour la façade nord de l'insula I - un mur aveugle - n'en serait que plus vraisemblable.

(45) Puisque la date probable de l'érection du mur 36 est plus ou moins contemporaine de la réfection de l'insula $\mathrm{I}$, après le milieu du ${ }_{11}{ }^{\mathrm{e}}$ siècle de notre ère.

(46) Contre cette hypothèse, il est juste de signaler que les fondations de ce mur sont profondes, quand celles du muret 36 , plus au nord, sont superficielles; mais le mur 51, que nous interprétons aussi comme un mur bahut est bien profondément fondé pour sa part...

(47) Rien ne permet en effet de dater les différents murs entrevus sur le boulevard (?) dans le secteur C1; quant aux indications fournies prar les remblais sous le béton de l'espace R, voir supra, n. 43.

(48) La cour en effet n'a pas été fouillèe et la connaissance que nous avons de tout le secteur nord-est de l'insula I est des plus sommaires.

(49) Pour la datation de ce remblai, voir l'article déjà cité de R. Guild, J. Guyon, L. Rivet (R. A. N., 1983), p. 18'́.

(50) L'hypothèse que la cathédrale antique d'Aix-en-Provence était une cathédrale double a été émise pour la première fois par P.-A. Fèvrier dans sa thèse, Le développement urbain en Provence des origines au XIVe siècle, Paris, 1964, p. 57 ; les nouvelles fouilles programmées en 1986 sur le site de Saint-Sauveur permettront peut-être de la vérifier.

(51) Pour l'histoire du palais à l'époque moderne, nous nous sommes fondés sur le travail de A. Poher, Le palais des archevêques d'Aix, du $X V^{\mathrm{e}}$ au $X V I I I^{\mathrm{e}}$ siècle, Mémoire de maitrise dactylographié, 1974. Il est regrettable que les résultats d'un tel travail soient restés confidentiels, malgré l'annonce d'une publication par le directeur de la recherche : J.-J. Gloton, Renaissance et baroque à Aix-en-Provence, Paris, 1979, note 117, p. 348. 
(52) P.-A. Février, Approche de villes médiévales de Provence, réflexions à partir de deux fouilles faites à Fréjus et à Aix, dans Rendiconti della Pontificia Accademia Romana di Archeologia, vol. LIII-I.IV (1980-1981, 1981-1982), p. 369-382 ; id., Aux origines de quelques villes médiévales du Midi de la Gaule, dans Rivista di Studi Liguri, vol. XI.IX, 1983 (1985), p. 316-335 (Atti del Congresso "I Liguri dall'Arno all'Ebro ").

(53) G. Démians d'Archimbaud, L'habitation rurale en Provence médiésale : techniques de construction et d'aménagement d'après des fouilles récentes, dans La construction au Moyen Age. IIistoire et archéologie, Paris, 1973, p. 59-122.

(51) II. Fixot, Nouvelles trouvailles de silos médiévaux en Provence, dans Provence IIistorique, fasc. 118, 1980, p. 38710' ; La conservation des grains à long terme, Colloque de Sénanque, 1977, Marseille, 1979.

(55) l. Boucharlat, .I. Colardelle, M. Fixot, J.-P. Pelletier, La céramique commune du XIe siècle dans le Sud-Fst de la France, dans La céramique médiévale en Méditerranée occidentale, $X^{\mathrm{e}}-\mathrm{X} V^{\mathrm{e}}$ siècles, Actes du colloque international de Val-

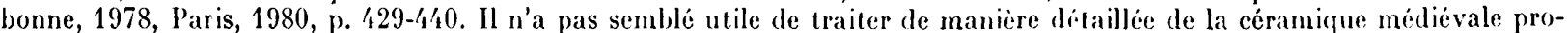
venant de cette fouille. Bin dehors de son intérêt chronologique qui sera exploité et discuté ici, ce matirriel ne prend son sens que par rapport aux collections en cours d'étude. Fous remercions L. Vallauri pour l'aide apportée à l'étude des productions de la fin du . Ioyen Age.

(56) G. I)émians d'Archimbaud, Les fouilles de Rougiers, Paris, 1981, p. 292-297.

(57) G. Démians d'Archimbaud, Les fouilles de Rougiers, op. cit., p. 297-302.

(58) G. Démians d'Archimbaud, Les fouilles de Rougiers, op. cit., p. 352-35t; mais des formes plus anciennes existent aussi : F. Boucharlat et alii, La céramique commune..., loc. cit., p. 4.37.

(59) P.-A. Février, M. Fixot, L. Rivet, Les fouilles des abords de la cathédrale de Fréjus, dans Provence IIistorique,

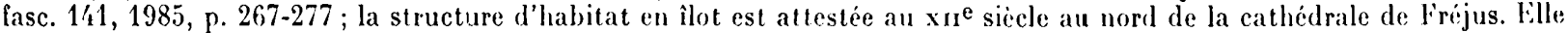
n'a pas ici pour origine l'adaptation lente d'une insula antique mais est críre de toutes pièces à l'emplacement d'une nécropole médiévale adjacente à la cathédrale. Il s'agit cependant d'un quartier de caractíre particulier, dépendance vraisemblable du quartier canonial ou épiscopal. De petites cellules d'habitation s'organisent autour d'une cour, mais il n'y a pas trace de répartition des fonctions par aile.

(60) R. Guild, Étude de la cathédrale d'Aix-en-Provence, Thèse pour le doctorat de troisième cycle, Aix, 1981.

(61) C'est-à-dire : "... les constructions de maisons et les terrains de Richard Barnoin et du chanoine Eudes, son fils, qui existent autour du cloître (claustrum) de Notre-Dame-du-Siège d'Aix, entre notre habitation et l'iglise Notre-1)ame, car l'archevêque Rostan, sur l'intervention de Richard, et à la prière de son fils Eudes, les a attribués et confirmés au susdit autel et aux chanoines qui habitent là et servent I)jeu ". ("'est la traduction proposie par L. J. Labande, Saint-Sauveur d'Aix, étude critique sur les parties romanes de cette cathédrale, dans Bulletin Archéologique du Comité des Travaux II istoriques et Scientifiques, 1912, p. 315.

(62) P.-A. Février, Le développement urbain en Provence de l'époque romaine à la fin du XIV'e siècle, Paris, 196'ı, p. 97-98.

(63) P.-A. Février, La cathédrale de Fréjus, Paris, 1981.

(64) Cl. Sintes, Les bâtiments canoniaux et la cité épiscopale d'Arles, Némoire de maîtrise dactylographié, 1979.

(65) J. Pourrière, Recherches sur la première cathédrale d'Aix-en-Provence, Paris, 1939. Il n'a pas paru utile de renvoyer à l'abondante bibliographie antéricure dont l'auteur fait précisément justice.

(66) R. Guild, J. Guyon, I. Rivet, Les origines du baptistère..., loc. cit., p. 199-201.

(67) Textes rassemblés dans la thèse de R. Guild, op. cit., p. 337-3'‘1.

(68) Sobolis, Catalogue historique des Seigneurs Archevêques d'Aix, ms. 10'46, Bibliothèque Méjanes, Aix.

(69) G. Démians d'Archimbaud, Les fouilles de Rougiers, op. cit., p. 318-327.

(70) Identification due à II. Bompaire.

(71) G. Démians d'Archimbaud, Les fouilles de Rougiers, op. cit., p. 237.

(72) G. Démians d'Archimbaud, Les fouilles de Rougiers, op. cil., p. 238-239.

(73) (j. Démians d'Archimbaud, Les fouilles de Rougiers, op. cit., p. 351-102.

(74) G. Démians d'Archimbaud, Les fouilles de Rougiers, op. cit., p. 26',$n^{0} 120$; fig. p. 27', $\mathrm{n}^{\circ} 120$. L'exemplaire recueilli en fouille a été reproduit dans .I. Fixot, J. Guyon, J.-P. Pelletier, L. Rivet, Les fouilles de la Cour de l'Archevêché, Aix, 1985, p. 42 et $46, n^{\circ} 37$.

(75) E. Boucharlat et alii, La céramique médiévale..., loc. cit., p. ' 136 , pl. I, nos 7 et 8 , pl. II, nos 1 et 1'́ line photographie de ces objets se trouve dans .I. Fixot, J. Guyon, J.-P. Pelletier, L. Rivet, Les fouilles de la Cour..., op. cit., p. 42 et 46.

(76) Les rapports de visite de 1708 et de 1729 qui établissent la fonclion de différentes pièces ont été transcrits par 1. Poher dans son travail (Archives des Bouches-du-Rhône, G 101 et G 102).

(77) Pitton, Annales de la Sainte Église d'Aix, Aix, 1668, attribuait les plus anciens vestiges du palais à une construction faite par l'archevêque Arnaud de Barchesio. La date proposée reposait sur la mauvaise interprétation du fameux texte par lequel une maison de la Rue Droite était échangée par un archevêque contre la maison du prévôt. Pitton avait situé cette transaction vers 1331, sous l'épiscopat d'Arnaud de Barchesio. Sobolis, Catalogue historique des Seigneurs Archevêques d'Aix, ms. 1046, Bibliothèque Néjanes, p. 527, critiquait déjà Pitton. Il reportait à 1424 et à l'archevêque $A$ vignon Nicholaï le "retour " des prélats dans le bourg Saint-Sauveur, tout en rappelant que l'on devait à $A$ rnaud de Barchesio "quelques réparations à ce palais qui était pour lors peu de choses, ayant été pour lors agrandi et élargi de beaucoup ". Ce texte laissait donc entendre que Sobolis déjà pensait à un édifice antérieur au xive siècle, mais sans l'identifier. l.a mêtme prudence archéologique a été observée depuis. J.-J. Gloton, Renaissance et baroque..., op. cit., rendant compte de la tradition gothique dans l'architecture aixoise et des chantiers de la fin du Moyen Age garde le silence à propos de ces parties anciennes du palais. Ce mutisme laisserait supposer qu'il n'attribue pas non plus la salle basse de l'aile nord à une campagne du courant $\mathrm{lu} \mathrm{xv}^{\mathrm{e}}$ siècle.

(78) Les différentes interprétations ont été exposées par R. Guild, Étude de la cathédrale..., op. cit., p. 303. 
(79) J. Pourrière, Recherches sur la première cathédrale..., op. cit., p. 170. p. $122-141$

(80) II. Pradalier, Le Palais de la Berbie, dans Congrès archéologique de France, $140^{\mathrm{e}}$ session, Albigeois, 1982 (1985),

(81) J.-L. Biget, Recherches sur le financement des cathédrales du Midi au XIII e siècle, dans La naissance et l'essor du gothique méridional au XIII ${ }^{\mathrm{e}}$ siècle, Cahiers de Fanjeaux, 9, Toulouse, 1974.

(82) F. Benoit, La cathédrale Saint-Sauveur, dans Congrès Archéologique de France, $95 \mathrm{e}$ session, Aix-en-Provence et Nice, 1932 (1933), p. 9-29.

(83) E. Viollet-le-Duc, Dictionnaire raisonné de l'architecture française du XIe au XVIe siècle, tome VII, p. 19-24 (art. Palais) ; Y. Carbonell-Lamothe, Recherches sur la construction du Palais Neuf des archevêques de Narbonne, dans Narbonne, Archéologie et histoire, Montpellier, 1973, tome II, p. 215-235.

(84) Signalons les trouvailles récentes à Apt, Antibes, Buoux, Marseille, Viviers, Vienne que nous nous étions proposées de réunir dans une publication à la suite d'un séminaire tenu en 1984 à l'Université de Provence.

(85) S. R. Guild, J. Guyon, I. Rivet, Les origines du baptistère..., loc. cit., p. 204-206. la cathédrale.

(86) C'est du moins ce que pense $M^{\text {me }}$ Muriel Vecchione dans l'étude qu'elle accomplit sur les parties gothiques de

(87) Sur ces techniques, voir par exemple P. Donati, Il campanato, Bellinzona, 1981.

(88) P. Pansier, Les palais cardinalices d'Avignon aux XIV et XVe siècles, Avignon, 1932 ; H. Aliquot, Les palais cardinalices hors les murs d'Avignon au XIV siècle, Thèse de doctorat de III e cycle, $A \mathrm{ix}, 1982$, exemplaire dactylographié, p. $390-394$.

(89) Photographie de l'objet dans M. Fixot, J. Guyon, J.-P. Pelletier, L. Rivet, Les fouilles de la Cour..., op. cit., p. 26. Pour la datation, voir G. Démians d'Archimbaud, Les fouilles de Rougiers..., op. cit., p. 318-326.

(90) J. Pourrière, Recherches sur la première cathédrale..., op. cit., p. 168 et suiv.

(91) N. Didier, II. Dubled, J. Barruol, Cartulaire de l'Église d'A pt, Paris, 1967, charte LIV, p. 182-183, note 's p. 183 ; nole 1 p. 286.

(92) Le vray pourtraict de la ville d'Aix-en-Provence, gravé en 1573 pour la Cosmographie Universelle de François de Belleforest, Paris, 1575.

(93) Pour la physionomie de ce quartier, il faut rappeler J. Duranti de la Calade, Notes sur les rues d'Aix, dans Annales de Provence, 1910, p. 39-44.

(94) C'est le mur crénelé, mais dépourvu cette fois de son couronnement, qui divise sans doute en deux parties la cour de l'archevêché sur le plan de J. Maret publié en 1624.

(95) J.-J. Gloton, Renaissance et baroque..., op. cit., p. 347-349.

(96) E. Marbot, Histoire de Notre-Dame-de-la-Seds, Aix, 1904 : "Pierre Filholi fit exécuter le grand escalier que l'on remania plus tard, son blason était à l'entrée ".

(97) J.-J. Gloton, Renaissance el baroque..., op. cit., p. 26, évoque ces galeries de bois ou de pierre dont sont friands les architectes.

(98) A. Poher, dans son travail, a résumé cette histoire. Nous remercions notre collègue Régis Bertrand pour la fiche rédigée à notre intention.

(99) L'exemple de la Cour de l'Archevêché d'Aix-en-Provence devrait montrer, espérons-le, que quoi qu'ait pu en écrire 13. Jestaz, Archéologie el architecture, dans Bulletin monumental, 143, 1985, p. 8, les notions de site et de monument sont, pour les archéologues (même " de terrain"), plus complémentaires que contradictoires...

Le directeur de la publication: Alatn Eriande-Brandenburg. 